\title{
Channel Estimation in Single Carrier Frequency Domain Equalization Space Division Multiple Access Systems
}

\author{
By \\ Fayyaz Ahmad Siddiqui
}

\begin{abstract}
A thesis submitted to the Faculty of Graduate Studies and Research in partial fulfillment of the requirement for the degree of
\end{abstract}

Doctor of Philosophy

Ottawa-Carleton Institute for Electrical and Computer Engineering

Faculty of Engineering

Department of Systems and Computer Engineering,

Carleton University

Ottawa, Ontario, Canada

Copyright $\odot$ Fayyaz Ahmad Siddiqui, 2008 


$\begin{array}{ll}\begin{array}{l}\text { Library and } \\ \text { Archives Canada }\end{array} & \begin{array}{l}\text { Bibliothèque et } \\ \text { Archives Canada }\end{array} \\ \begin{array}{l}\text { Published Heritage } \\ \text { Branch }\end{array} & \begin{array}{l}\text { Direction du } \\ \text { Patrimoine de l'édition }\end{array} \\ \begin{array}{l}\text { 395 Wellington Street } \\ \text { Ottawa ON K1A 0N4 } \\ \text { Canada }\end{array} & \begin{array}{l}\text { 395, rue Wellington } \\ \text { Ottawa ON K1A 0N4 } \\ \text { Canada }\end{array}\end{array}$

Your file Votre référence ISBN: 978-0-494-40537-6 Our file Notre référence ISBN: 978-0-494-40537-6

NOTICE:

The author has granted a nonexclusive license allowing Library and Archives Canada to reproduce, publish, archive, preserve, conserve, communicate to the public by telecommunication or on the Internet, loan, distribute and sell theses worldwide, for commercial or noncommercial purposes, in microform, paper, electronic and/or any other formats.

The author retains copyright ownership and moral rights in this thesis. Neither the thesis nor substantial extracts from it may be printed or otherwise reproduced without the author's permission.
AVIS:

L'auteur a accordé une licence non exclusive permettant à la Bibliothèque et Archives Canada de reproduire, publier, archiver, sauvegarder, conserver, transmettre au public par télécommunication ou par l'Internet, prêter, distribuer et vendre des thèses partout dans le monde, à des fins commerciales ou autres, sur support microforme, papier, électronique et/ou autres formats.

L'auteur conserve la propriété du droit d'auteur et des droits moraux qui protège cette thèse. $\mathrm{Ni}$ la thèse ni des extraits substantiels de celle-ci ne doivent être imprimés ou autrement reproduits sans son autorisation.
In compliance with the Canadian Privacy Act some supporting forms may have been removed from this thesis.

While these forms may be included in the document page count, their removal does not represent any loss of content from the thesis.
Conformément à la loi canadienne sur la protection de la vie privée, quelques formulaires secondaires ont été enlevés de cette thèse.

Bien que ces formulaires aient inclus dans la pagination, il n'y aura aucun contenu manquant.

\section{Canada}




\section{Abstract}

Single Carrier-Frequency Domain Equalization (SC-FDE) has been shown to be a favorable choice for uplink communication. In an uplink SC-FDE, Space Division Multiple Access (SDMA) cellular system, a receiver has to deal with inter-user interference along with the Inter-Symbol Interference (ISI). Inter-user interference can arise by two kinds of users: In-cell co-channel Users (ICUs) and Out of Cell co-channel Interferers (OCIs) from neighboring cells. This thesis presents a combination of iterative channel equalization and Channel Estimation (CE) algorithms for mobile users in such interference-prone SC-FDE uplink systems.

For equalization purposes, a low complexity multi-user Soft Decision-based Iterative Block Decision Feedback Equalization (SD-IBDFE) is introduced in which at each iteration estimated co-channel and inter-symbol interference, derived from soft detector outputs, is subtracted. It is shown that this soft subtraction yields substantially lower error probability for frequency selective multi-user channels. Utilizing the SD-IBDFE, a low complexity threshold-based Hard Decision-based Decision Feedback Iterative Channel Estimation (DFICE) algorithm (HD-DFICE) is proposed.

Adaptive suppression of the out-of-cell interference can be done without explicitly estimating out-of-cell interference data or channels by Least Squares (LS) processing over a series of successive Fast Fourier Transform (FFT) blocks. Based on this, an interference suppression algorithm (LS-IS) is proposed in which it is shown that the SDIBDFE with Parallel Interference Cancellation (PIC) and the LS adaptation algorithm give significant improvement in DFICE to estimate the in-cell SDMA users and their data in an iterative manner. Also we show that iterative $\mathrm{CE}$ over multiple frames to estimate the desired user's channels provides some additional gain at slightly higher complexity. We also present a DFICE technique using soft estimates of the decisions derived from the Low-Density Parity-Check (LDPC) code-based Turbo Equalization (TE). In Soft Decision-based DFICE (SD-DFICE) the extrinsic information is used in the soft mapping of the symbols. While deriving the SD-DFICE via an Expectation Maximization (EM) algorithm we also provide a rationale to justify the use of threshold in DFICE algorithm. 
The proposed SD-IBDFE assisted DFICE is also applied to suppress the multi-user interference in a SC-FDE power efficient chunk-based uplink system B-IFDMA defined under the WINNER ${ }^{1}$ concept. For comparison purposes the CE performance of BIFDMA is compared with the full bandwidth and a Local Frequency Division Multiple Access (L-FDMA) system. Finally, we present methods like time domain processing and overlap-cut method to deal with the asynchronous OCIs.

\footnotetext{
${ }^{1}$ WINNER-Wireless World Initiative New Radio is a consortium of 41 members coordinated by Nokia-Siemens Networks. https://www.ist-winner.org/
} 
To My Mother...

Mom Pop Mom 


\section{Acknowledgements}

First of all I would like to thank almighty Allah who gives us strength to achieve objectives in our lives. I want to thank my supervisors David Falconer and Florence Danilo-Lemoine for their invaluable and in-depth help, suggestions and guidance throughout this thesis. Especially, a bundle of thanks to David for his advices, patience and moral support.

I also want to thank my friends Chan Tong Lam, Mahmud-ur-Rehman and Maryam Sabbaghian for making the work on the WINNER project a memorable one. I also want to thank the ladies in the System's office, Blazanka power, Darlene Hebert and Anna Lee who always provided all the necessary paper work in time.

Finally, I am thankful to my parents, Aagee and mom, the two BJs, my brother Riaz and sister Sumaira. Also I want to acknowledge my beloved wife Farheen and my kids Iman and Saad for their continuous love and support.

Fayyaz Ahmad Siddiqui

Ottawa, Ontario,

Canada.

April 2008. 


\section{Table of Contents}

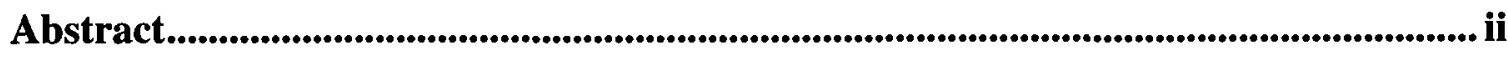

Acknowledgements ............................................................................................................................. iv

Table of Contents .............................................................................................................................. v

List of Figures......................................................................................................................................... xi

List of Tables ........................................................................................................... xvii

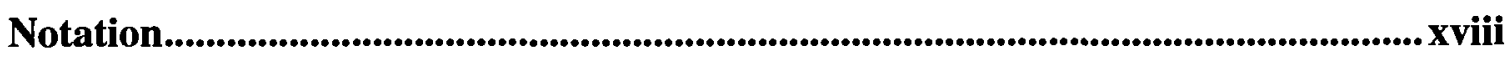

List of Acronyms .................................................................................................................... xix

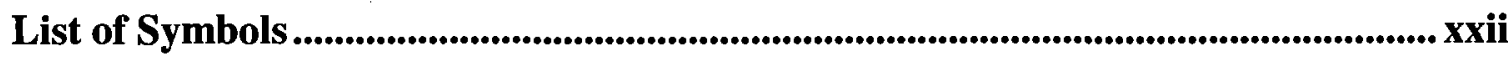

Chapter 1 ..................................................................................................................................... 1

Introduction ......................................................................................................................................... 1

1.1. Thesis Objectives ............................................................................................. 1

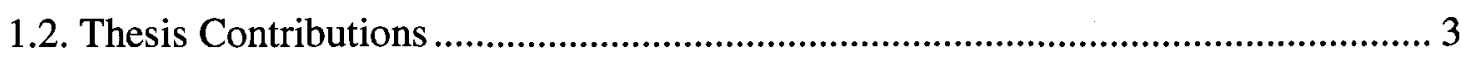

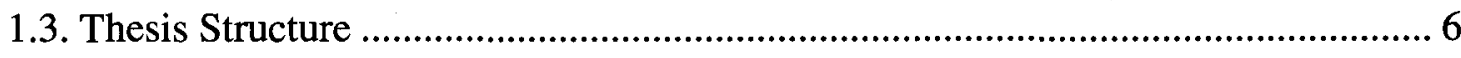

Chapter 2 ..................................................................................................................................................... 10

Frequency Domain Processing in Uplink SDMA Cellular Systems................................ 10

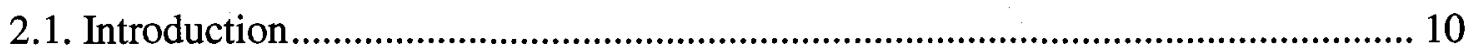

2.2. Single Carrier Frequency Domain Equalization (SC-FDE)..................................... 10

2.2.1. Initial Work on SC-FDE ............................................................................ 11

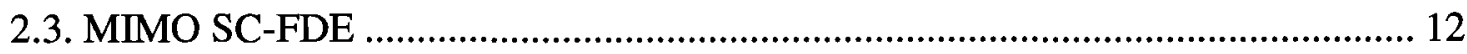

2.4. Multi-User Channel Estimation ............................................................................ 13

2.4.1. Previous Work on MIMO Channel Estimation ................................................. 13

2.5. SC-FDE SDMA Signal and Channel Model ........................................................ 15

2.5.1. Generalized Multi-Carrier (GMC) SDMA Transceiver Structure.................... 15

2.5.2. Rayleigh Faded Doubly Selective Channel Model............................................. 18

2.6. Frequency Domain Linear Equalization and Adaptation.......................................... 21

2.6.1. Direct Adaptation using Decisions or Pilots................................................... 22

2.6.2. Indirect Adaptation with Perfect (Known) Channel State Information ........... 23

2.6.3. Indirect Adaptation using Estimated CFR ...................................................... 24

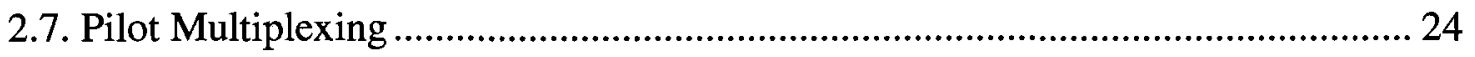


2.7.1. Time Multiplexed Training (TMT) ............................................................ 25

2.7.2. Frequency Multiplexed Training (FMT) ......................................................... 26

2.7.3. Comparison of TMT and FMT ……………………................................ 27

2.7.4. Optimal Training Sequence ......................................................................... 27

2.7.5. Initial Channel Estimates ............................................................................. 28

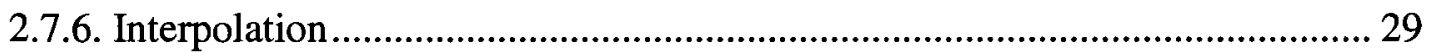

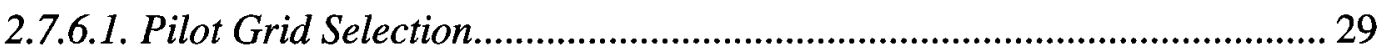

2.7.6.2. FFT/IFFT Interpolation and Smoothing................................................. 30

2.7.6.3. 2xID Wiener Interpolation ………………………………................... 33

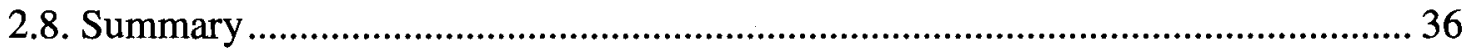

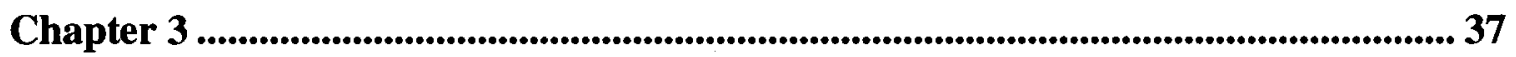

Pilot Multiplexing in SC-FDE with Intra-Cell Interference ...........................................37

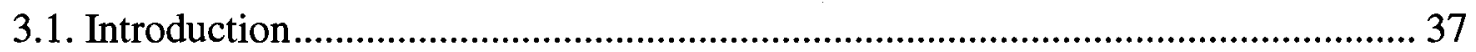

3.2. Inter-User Pilot Co-ordination in SC-FDE with Intra-Cell Interference ............... 37

3.2.1. Overlapping Training.................................................................................... 38

3.2.1.1. Least Squares (LS) Multi-User CE for Non-Orthogonal Training .......... 39

3.2.2. Orthogonal Training..................................................................................... 41

3.2.2.1. Orthogonal Time Multiplexed Training (TMT-ortho) ............................... 41

3.2.2.2. Orthogonal Frequency Multiplexed Training (FMT) .............................. 42

3.2.2.3. Pilot Overhead Definition ...................................................................... 43

3.2.2.4. Least Squares (LS) Multi-User CE for Orthogonal Training ................... 43

3.3. Comparison between Orthogonal and Overlapping Training.................................. 44

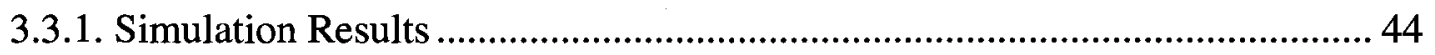

3.3.1.1. Comparison between TMT and FMT ................................................... 46

3.3.1.2. Comparison between Interpolation Choices and Complexities................ 48

3.3.1.3. Time Averaging in TMT ......................................................................... 50

3.3.1.4. CE and Vehicular Speeds..................................................................... 50

3.4. BER Analysis with Channel Estimation ............................................................... 51

3.4.1. Analysis of CE Error in Multi-User Scenario................................................. 52

3.4.2. Mean Squared Error (MSE) Analysis ......................................................... 56

3.4.3. Comparison of Analytical and Simulation Results............................................ 57 


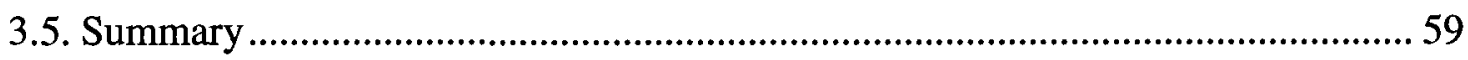

Chapter 4 ......................................................................................................................................6 60

Inter-Cell Interference in SC-FDE SDMA Systems .............................................................60

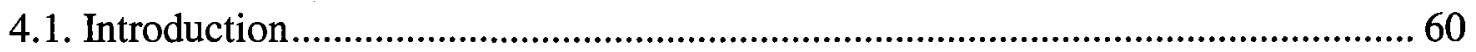

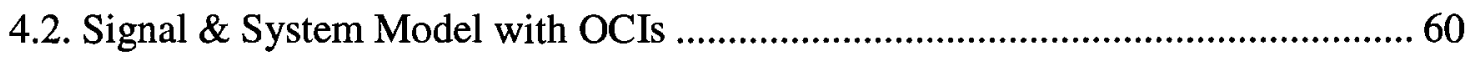

4.2.1. Spatial Distribution of OCIs ............................................................................. 61

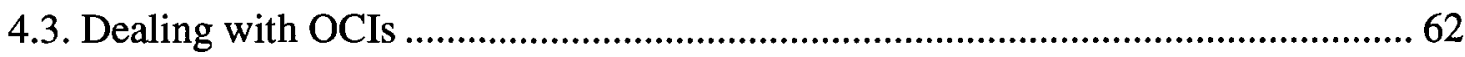

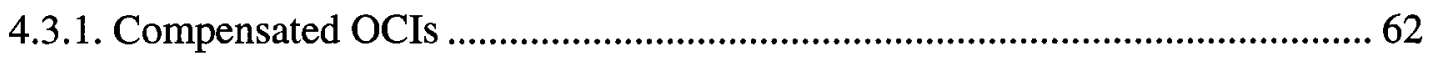

4.3.2. Uncompensated OCIs ................................................................................... 64

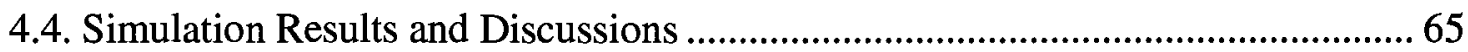

4.4.1. Comparison between Compensated and Uncompensated OCIs ....................... 66

4.4.2. Uncompensated OCIs with no Intra-cell Interference ........................................ 68

4.4.3. Uncompensated OCIs with Intra-cell Interference ............................................ 72

4.5. Modeling of OCIs with a Gaussian Approximation (GA) ...................................... 74

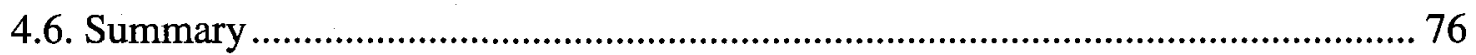

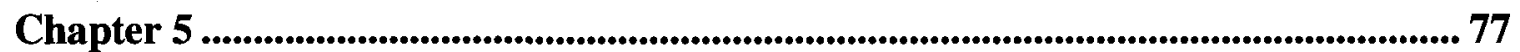

MIMO Soft Decision-based Iterative Block DFE ......................................................... 77

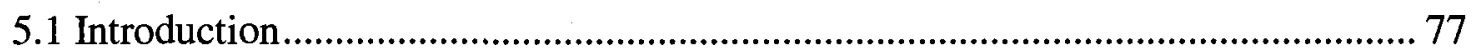

5.2. Multi-User Soft Decision-based Iterative Equalization.......................................... 77

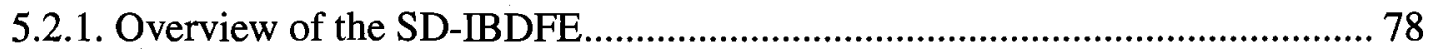

5.2.2. SD-IBDFE Coefficients Computation ....................................................... 79

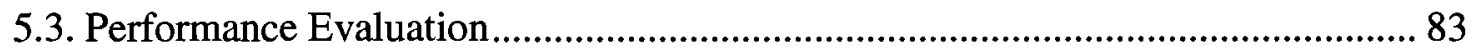

5.3.1. SD-IBDFE Performance Evaluation with no Intra-cell Interference................ 83

5.3.2. SD-IBDFE Performance Evaluation with Intra-cell Interference.................... 84

5.3.3. SD-IBDFE Performance Evaluation with Intra and Inter-cell Interference ... 85

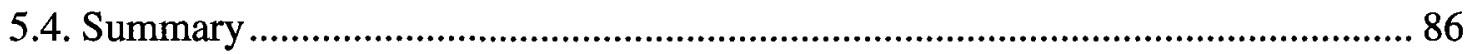

Chapter 6 ................................................................................................................................ 88

Interference Suppression in SC-FDE SDMA System........................................................ 88

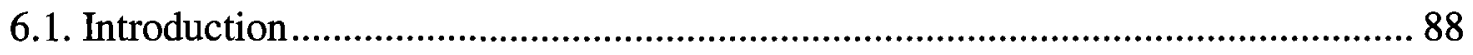

6.2. Decision Feedback Iterative Channel Estimation (DFICE) ..................................... 88

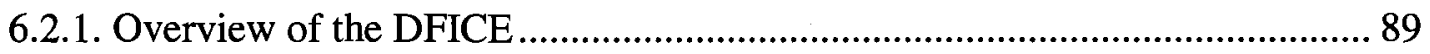




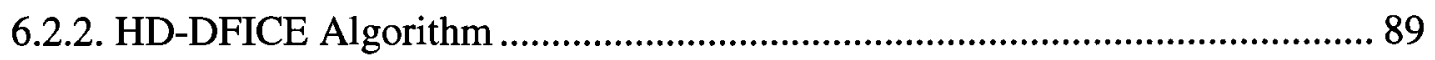

6.3. Simulation Results ............................................................................................ 91

6.3.1. HD-DFICE Performance Evaluation with no Multi-User Interference........... 92

6.3.2. HD-DFICE Performance Evaluation with Inter-Cell Interference .................. 93

6.4. Parallel Interference Cancellation (PIC) assisted HD-DFICE................................... 96

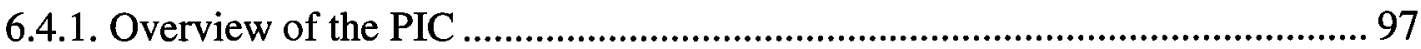

6.4.2. PIC-assisted HD-DFICE Algorithm ……………….......................................... 97

6.5. Simulation Results ............................................................................................. 98

6.5.1. PIC-assisted HD-DFICE Performance Evaluation with Intra and Inter-cell

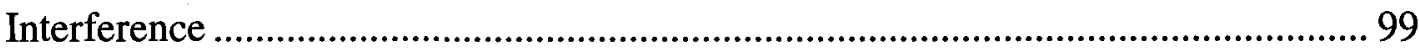

6.6. Interference Mitigation via LS-IS Algorithm ...................................................... 101

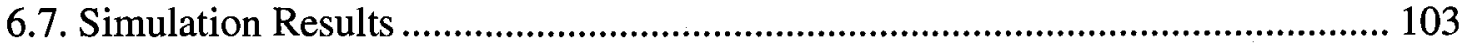

6.8. Optimizing Pilot Overhead with LS-IS algorithm ................................................ 104

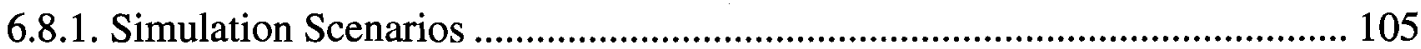

6.9. Overall Comparison of the Algorithms.................................................................... 107

6.10. Channel Estimation over Multiple Frames ............................................................ 109

6.11. Summary ……………................................................................................ 111

Chapter 7 ..........................................................................................................................1113

Turbo Equalization based Iterative Channel Estimation .................................................. 113

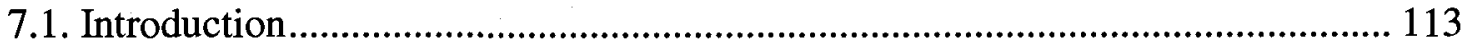

7.2. Overview of Frequency Domain Turbo Equalization.......................................... 113

7.2.1. Low-Density Parity-Check (LDPC) code-based Turbo Equalization ........... 114

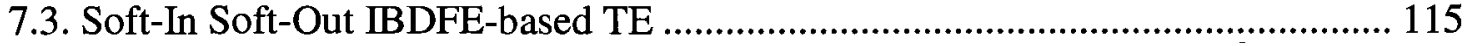

7.4. TE with ICE and Interference Suppression ......................................................... 118

7.5. Frequency Domain TE-based HD-DFICE ........................................................... 119

7.5.1. TE-Modified WINNER Frame Structure, Simulation Assumptions and System

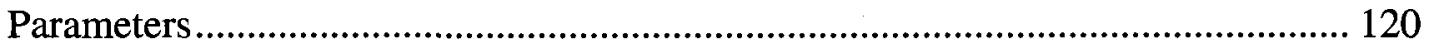

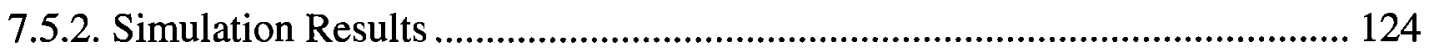

7.6. Concluding Remarks on TE with HD-DFICE ……............................................ 128

7.7. TE-assisted Sub-Space Interference Suppression (SS-IS) .................................... 129

7.7.1. SS-IS Algorithm........................................................................................... 129 


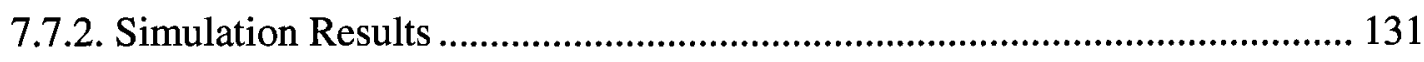

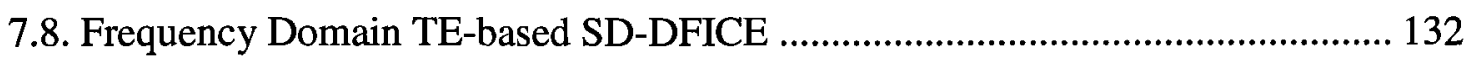

7.8.1. Derivation of SD-DFICE using EM Algorithm.............................................. 133

7.8.2. Simulation Results ..................................................................................... 135

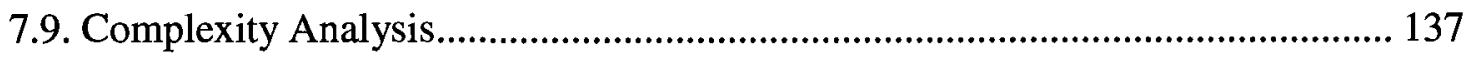

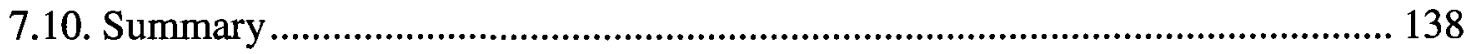

Chapter 8 ......................................................................................................................................... 139

Chunk-Based Transmission in SC-FDE SDMA systems ................................................... 139

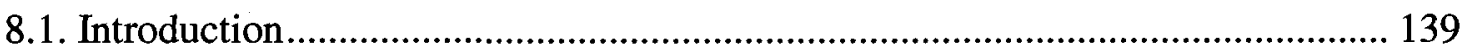

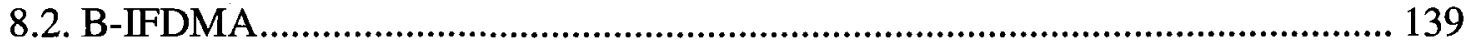

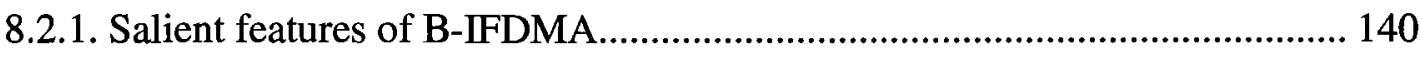

8.3. B-IFDMA Structure and System Parameters.......................................................... 141

8.3.1. Pilot-assisted CE in B-IFDMA ……............................................................. 141

8.4. SD-IBDFE Performance Evaluation............................................................. 145

8.5. DFICE-Smoothing via Averaging .................................................................... 146

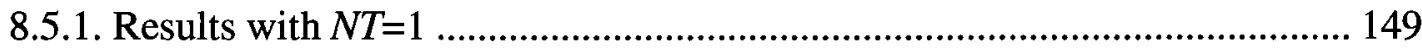

8.6. CE over Multiple Frames................................................................................ 151

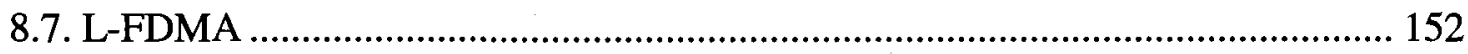

8.7.1. Pilot Arrangements and DFICE Performance............................................. 153

8.7.2. Simulation Results ..................................................................................... 153

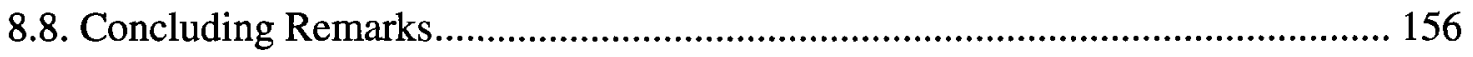

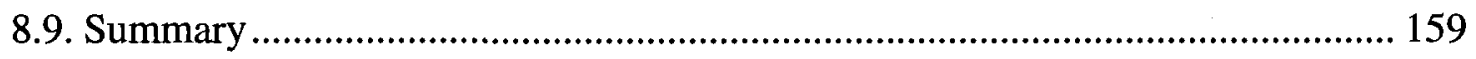

Chapter 9 ............................................................................................................................. 161

Conclusions and Future Work..................................................................................... 161

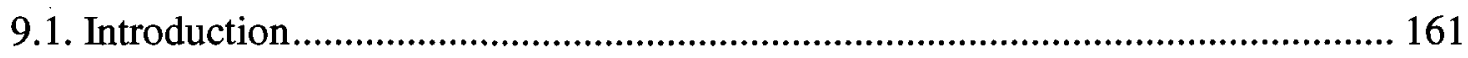

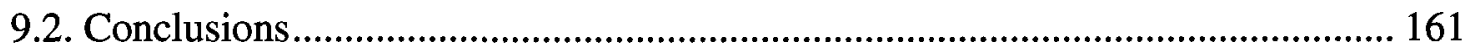

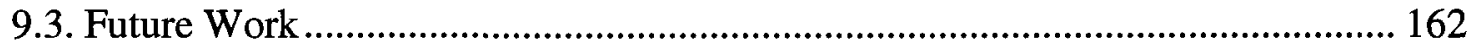

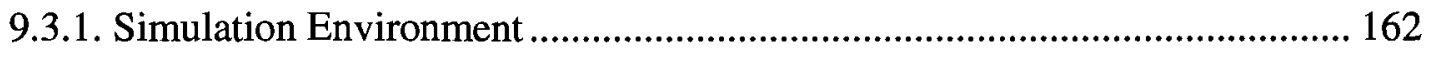

9.3.2. Comparison of Proposed SS-IS and LS-IS with more Complex Optimization

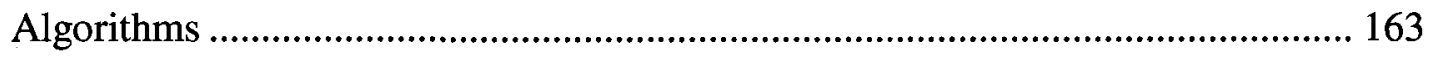

9.3.3. Transmitter Adaptation ................................................................................ 163 
9.3.4. Cross-Layer Efforts to Reduce Interference ………........................................ 163

9.3.5. Multi-User BERT Chart Analysis for TE ...................................................... 164

9.3.6. BER Expression in the Presence of Interference ............................................ 164

Appendix A ....................................................................................................................... 165

A.1. Proposed Uplink Interference Simulation Scenario............................................... 165

A.1.1. Suggested OCI Power Levels ........................................................................ 166

Appendix B .................................................................................................................................. 168

B.1. Asynchronous Out of Cell Interferers (AOCIs) .................................................. 168

B.1.1. Effect of Asynchronism ............................................................................ 169

B.1.2. Suppression of AOCI ............................................................................ 173

B.1.2.1. Space-Time Processing to Align AOCI ................................................ 173

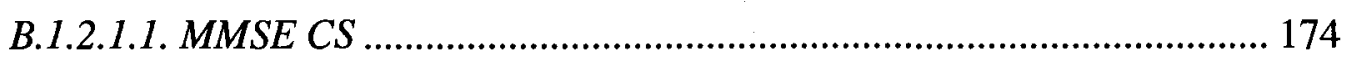

B.1.2.1.2. Minimizing the Residual ISI ........................................................ 175

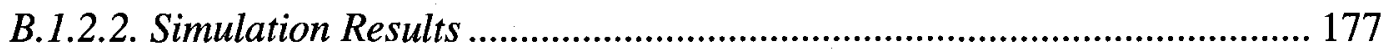

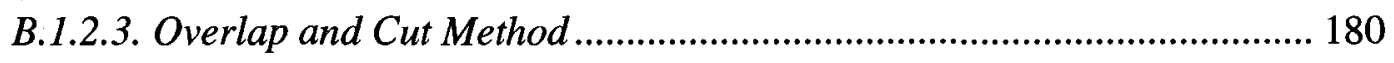

B.2. Conclusions .................................................................................................... 181

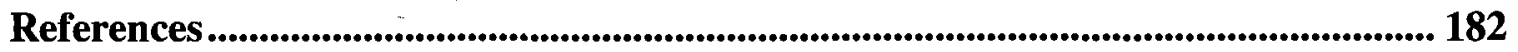




\section{List of Figures}

Fig. 1.1. Distribution of intra and inter-cell interference................................................. 2

Fig. 2.1. Block diagram of a coded SC-FDE transceiver................................................ 11

Fig. 2.2. Block diagram of a GMC structure-based SC-FDE transceiver.......................... 17

Fig. 2.3. A multi-user multi-path channel model................................................................ 18

Fig. 2.4. Snapshot of time-frequency variation of the channel (one frame with $F=120$ ). 20

Fig. 2.5. Snapshot of time-frequency variation for disjoint transmission (two frames each

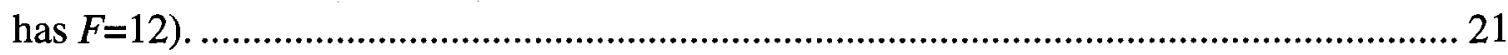

Fig. 2.6. Concept of Time Multiplexed Training (TMT)................................................. 25

Fig. 2.7. Concept of Frequency Multiplexed Training (FMT) .........................................26

Fig. 2.8. Single user pilot grid (a frame).......................................................................... 29

Fig. 2.9. Re-growth in the estimated CIR. .................................................................... 31

Fig. 2.10. Effect of filtering on the estimated CIR. ………............................................ 32

Fig. 2.11. Actual and estimated CFR with and without LP smoothing filter. .................. 32

Fig. 2.12. Exploded view of the actual and estimated CFR with and without LP

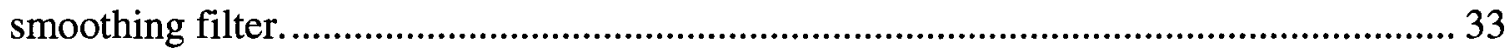

Fig. 3.1. Non-Orthogonal Time Multiplexed Training (TMT-Chu with three users)....... 38

Fig. 3.2. Orthogonal Time Multiplexed Training (TMT-ortho with three users)............. 41

Fig. 3.3. Concept of Frequency Multiplexed Training (FMT) with three users............... 42

Fig. 3.4. Coded average BER performance, $M=K=2, N T=2$ and 3, LE - Performance of orthogonal FMT Vs Overlapping TMT with QPSK random data symbols as training (Perfect Channel State Information (PCSI) curve shown for reference).......................... 47

Fig. 3.5. Comparison of interpolation choices (FMT, $M=K=1$ ) in terms of average MSE.

Fig. 3.6. Comparison of interpolation choices (FMT, $M=K=1$ )- coded average BER. .... 49

Fig. 3.7. Comparison of interpolation complexities (FMT, $M=K=1$ )- coded average BER.

Fig. 3.8. Effect of time averaging on coded average BER (TMT-Chu), $N T=1$ and 2, LE.50 
Fig. 3.9. Coded average BER performance, effect of different Doppler on pilot

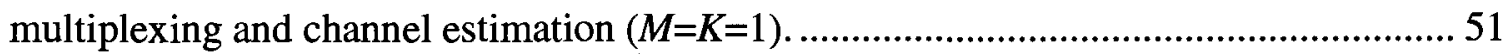

Fig. 3.10. Average uncoded BER, Analytical Vs Simulation, $M=K=1$, LE with PCSI.... 52 Fig. 3.11. a) Histogram showing the validity of the Gaussian approximation of the equalizer output with no channel estimation errors (PCSI)- $M=K=2$, Uncoded-LE. b) Corresponding CDF.

Fig. 3.12. a) Histogram showing the validity of the Gaussian approximation of the equalizer output with channel estimation errors- $M=K=2$, Uncoded-LE. b) Corresponding CDF. 55

Fig. 3.13. Average BER-Gaussian approximation of the equalizer output with channel estimation errors and PCSI- $M=K=2$, Uncoded-LE. 58

Fig. 3.14. Average BER-Gaussian approximation of the equalizer output with channel estimation errors and PCSI- $M=K=3$, Uncoded-LE................................................... 58

Fig. 4.1. One compensated OCI (FMT example). ........................................................ 63

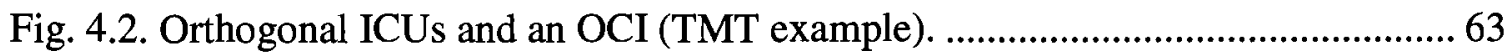

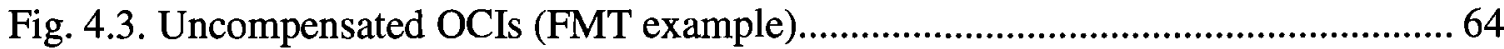

Fig. 4.4. Average BER-Uncompensated and compensated OCI, OCI training known or unknown (relative power=1/16), $M=3, K=2$.

Fig. 4.5. Average BER-Compensated OCI, OCI training known or unknown (relative

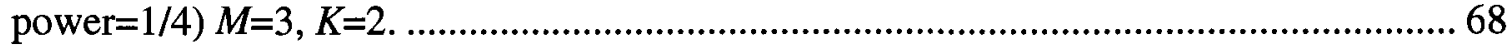

Fig. 4.6. Overlapping OCIs $(K=1, P=2)$.

Fig. 4.7. Average BER Comparison between known and unknown training of overlapping OCIs, $M=3, K=1, P=2$ (relative power=1/4).

Fig. 4.8. Average BER- Performance with diversity and known and unknown training,

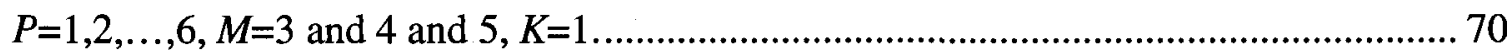

Fig. 4.9. Average BER-Effect of distance attenuation of OCIs, $P=4, M=3, K=1 \ldots \ldots \ldots . .71$ Fig. 4.10. Average BER-Different power levels of OCIs (Uncompensated OCIs- no intracell interference), $P=1,2, \ldots, 6, M=2, K=1, N T=2$ and $(K+P)$. 72

Fig. 4.11. Different cases of appearance of ICUs' and OCIs' training blocks (TMT).... 73 Fig. 4.12. Average BER- Different cases of appearance of OCIs (Uncompensated OCIs with intra-cell interference) 
Fig. 4.13. Average MSE at the LE output with PCSI, OCI modeling with GA ( $P=5$ @$18.2 \mathrm{~dB})$

Fig. 4.14. Average MSE at the LE output with PCSI, OCI modeling with GA ( $P=6$ @18.2dB) 76

Fig. 5.1. Block diagram of SD-IBDFE. 79

Fig. 5.2. PCSI Vs FMT-Chu, improvement in average FER through SD-IBDFE, $M=K=1$.

Fig. 5.3. Average BER comparison between $\operatorname{LE}\left(I_{\mathrm{E}}=0\right)$ and $\operatorname{SD}-\operatorname{IBDFE}\left(I_{\mathrm{E}}=4\right), M=K=2.85$

Fig. 5.4. Scenario of the overlapping OCIs' and ICUs' training blocks. 85

Fig. 5.5. Average BER-Improvement by SD-IBDFE (Uncompensated OCIs with intra-

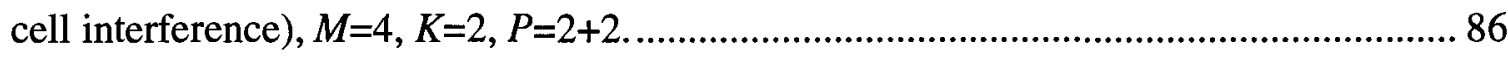

Fig. 6.1. Block diagram of hard decision-based CE (HD-DFICE) .............................. 90 Fig. 6.2. Improvement in average BER via $\mathrm{HD}$-DFICE, $M=K=I_{I C E}=1, I_{E}=4, \mathrm{C} 2$-Channel, $50 \mathrm{~km} / \mathrm{hr}, N T=1$, results with LE and SD-IBDFE also shown for comparison. 92

Fig. 6.3. Average MSE with only LE, SD-IBDFE and SD-IBDFE with HD-DFICE (Uncompensated OCIs no Intra-cell interference) $M=2, K=I_{I C E}=1, I_{E}=4, P=2$ or $3 @-15 \mathrm{~dB}$, C2-Channel, $50 \mathrm{~km} / \mathrm{hr}, N T=2$. 94

Fig. 6.4. Average FER with only LE, SD-IBDFE and SD-IBDFE with HD-DFICE (uncompensated OCIs no intra-cell interference) $M=2, K=I_{I C E}=1, I_{E}=4, P=2$ or $3 @-15 \mathrm{~dB}$, C2-Channel, $50 \mathrm{~km} / \mathrm{hr}, N T=2$. 94

Fig. 6.5. Average FER with only LE and SD-IBDFE with HD-DFICE (uncompensated OCIs with no intra-cell interference @- $15 \mathrm{~dB}$ or $-16.6 \mathrm{~dB}) M=2, K=I_{I C E}=1, I_{E}=4$, C2Channel, $50 \mathrm{~km} / \mathrm{hr}, N T=2$.

Fig. 6.6. Average BER with only LE and SD-IBDFE with HD-DFICE (uncompensated OCIs with no intra-cell interference $@-15 \mathrm{~dB}$ or $-16.6 \mathrm{~dB}$ ), $M=2, K=I_{I C E}=1, I_{E}=4, \mathrm{C} 2$ Channel, $50 \mathrm{~km} / \mathrm{hr}, N T=2$ or $K+P$. 96

Fig. 6.7. Average FER with LE processing (uncompensated OCIs with inter and intra-cell interference), $M=4, K=2, \mathrm{C} 2-\mathrm{Channel}, 50 \mathrm{~km} / \mathrm{hr}, N T=2, P=4 @-15 \mathrm{~dB}$ and $-18.2 \mathrm{~dB}$... 100 Fig. 6.8. Average FER with SD-IBDFE and PIC assisted HD-DFICE (uncompensated OCIs with inter and intra-cell interference), $M=4, K=2$, C2-Channel, $50 \mathrm{~km} / \mathrm{hr}, N T=2,101$ $P=4 @-15 \mathrm{~dB}$ and $-18.2 \mathrm{~dB}$. 101 
Fig. 6.9. Block diagram of iterative receiver for mitigating intra and inter-cell interference using LS forward filtering (LS-IS algorithm). 102

Fig. 6.10. Average FER with SD-IBDFE and LS-IS assisted HD-DFICE (uncompensated OCIs with inter and intra-cell interference), $M=4, K=2$, C2-Channel, $50 \mathrm{~km} / \mathrm{hr}, \ldots \ldots \ldots . .104$ $N T=2, I_{\mathrm{ICE}}=1$ and $2, P=4 @-15 \mathrm{~dB}$. 104

Fig. 6.11. Average FER with SD-IBDFE and LS-IS assisted HD-DFICE (uncompensated OCIs with inter and intra-cell interference), $M=4, K=2, \mathrm{C} 2$-Channel, $50 \mathrm{~km} / \mathrm{hr}, N T=1$ and 2, $P=4 @-15 \mathrm{~dB}$, a) LE with 0 and 4 OCIs (non-iterative CE), b) PIC assisted HD-DFICE with 0 and 4 OCI and $I_{I C E}=1$ or 3 (no LS-IS), c) LS-IS based HD-DFICE with 0 and 4 OCI and $I_{I C E}=1$ and 3 . 106

Fig. 6.12. Multi-frame (full-duplex) CE structure $\left(F_{\mathrm{r}}=2\right)$. 110

Fig. 6.13. Average FER comparison with $F_{\mathrm{r}}=1$ or 2 , non-iterative CE SD-IBDFE and LSIS assisted HD-DFICE , $M=4, K=2, I_{I C E}=2$, C2-Channel, $50 \mathrm{~km} / \mathrm{hr}, N T=2, P=4 @-15 \mathrm{~dB}$.

Fig. 7.1. Simplified Block diagram of LDPC code-based TE. 116

Fig. 7.2. Simplified implementation diagram of an LDPC-based TE with WINNER frame structure. 123

Fig. 7.3. Average FER-Comparison of different algorithms with TE with $I_{\mathrm{ICE}}=1, I_{\mathrm{E}}=4$, $K=2, P=4 @-15 \mathrm{~dB}, N T=1$ and $2, M=3$ and $T_{r}=384$. 125

Fig. 7.4. Average FER-Comparison of different algorithms with TE with $I_{\mathrm{ICE}}=1$ and $2, I_{\mathrm{E}}$ $=4, K=2, P=4, N T=1$ and $2, M=3$ and $T_{r}=384$. 126

Fig. 7.5. Average FER-Comparison of different algorithms with TE with $I_{\mathrm{ICE}}=1$ and $2, I_{\mathrm{E}}$ $=4, K=2, N T=1$ and $2, P=4, M=3$ and $T_{r}=288$ and 384 .

Fig. 7.6. Average FER-Comparison of different algorithms with TE with $I_{\mathrm{ICE}}=1$ and $2, I_{\mathrm{E}}$ =4, $K=2, P=4, M=3$ and 4 and $T_{r}=288$ and 384 . 128

Fig. 7.7. Average FER-Comparison of LS-IS and SS-IS with TE and DFICE, $I_{\mathrm{E}}=4, I_{\mathrm{ICE}}$ $=1, N T=2, M=4$ and $T_{r}=288$. 131

Fig. 7.8. Simplified block diagram of soft decision based DFICE. 132

Fig. 7.9. Average FER-Comparison of hard decision and soft decision-based DFICE, SSIS and LS-IS with TE, $I_{\mathrm{ICE}}=1,2$ and $3, N T=2, F_{\mathrm{r}}=1, M=4=I_{\mathrm{E}}, P=0$ and $T_{r}=288$. 136 
Fig. 7.10. Average FER-Comparison of hard decision and soft decision-based DFICE, SS-IS and LS-IS with TE, $I_{\mathrm{ICE}}=1,2,3$ and $4, N T=2, I_{\mathrm{E}}=4$ and $5, F_{\mathrm{r}}=1, M=P=4 @-15 \mathrm{~dB}$ and $T_{r}=288$.

Fig. 8.1. Pilot arrangement for a B-IFDMA chunk-based system with one pilot per user per block-(F1-12). 142

Fig. 8.2. Actual Vs estimated disjoint CFR (straight horizontal lines represent the estimation using F1-12 pilot scheme).

Fig. 8.3. Pilot arrangement for a B-IFDMA chunk-based system with two pilot per user per block -(F2-12). 143

Fig. 8.4. Pilot distribution in a frame of B-IFDMA (concept of $N T=4$, a combination of TMT and FMT).

Fig. 8.5. Average FER-Comparison for B-IFDMA with $4 \times 3$ block size, CE using pilots and 2x1D interpolation, SD-IBDFE with no DFICE, $M=4, N T=2$ and 4 . 146

Fig. 8.6. Average FER-Comparison for B-IFDMA with 4x3 block size. 147

Fig. 8.7. Effect of code block size on average FER 148

Fig. 8.8. Average FER results with B-IFDMA - with or without OCIs @-15dB ( $N T=1,2$ and 4). 149

Fig. 8.9. Average FER results with B-IFDMA - with or without OCIs @-15dB. 150

Fig. 8.10. Average FER results with B-IFDMA - with or without OCIs @-15dB $(N T=1)$.

Fig. 8.11. Channel estimation over multiple frames in B-IFDMA............................. 152

Fig. 8.12. Pilot arrangement for an L-FDMA chunk-based system............................ 153

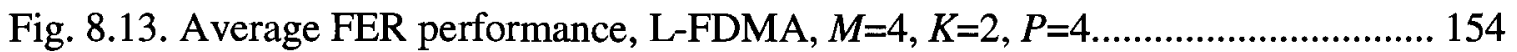

Fig. 8.14. Average FER comparison between B-IFDMA-64, B-IFDMA-128 and LFDMA-32, L-FDMA-64, L-FDMA-128, $M=K=N T=1$, a) B-IFDMA b) L-FDMA...... 156

Fig. A.1. Cellular layout, showing frequency reuse zones ..................................... 166

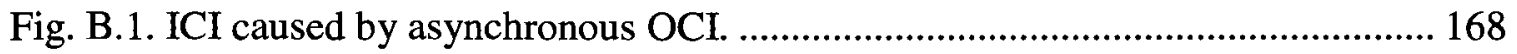

Fig. B.2. Average power-delay profile for SUI-5 model. .......................................... 169

Fig. B.3. a) SC-FDE-Average BER-Synchronous OCI with different power levels, $M=2$, $d_{O C F}=0, K=P=1 @ 0,5,10,15$ and $20 \mathrm{~dB}$ below the ICU. b) OFDM-Average BER (specifications are the same as in part a). 170 
Fig. B.4. a) SC-FDE-Average BER-Asynchronous OCIs with different power levels, $M=2, d_{O C F}=60, K=Z=1$. b) OFDM-Average BER (specifications are the same as in part a).

Fig. B.5. MMSE time domain equalization for channel truncation............................ 174

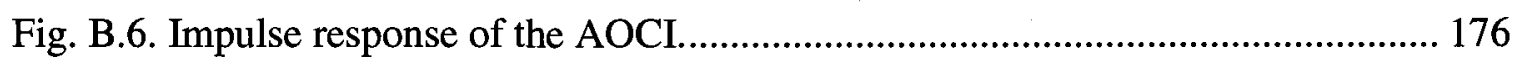

Fig. B.7. Average BER-Effect of low power AOCIs' CS, $M=2$ and $3, K=P=Z=1 @-15 \mathrm{~dB}$.

Fig. B.8. a) SC-FDE-Average BER-Effect of higher power AOCIs' CS, $M=2, K=P=Z=1$

@0dB. b) OFDM-Average BER (specifications are the same as in part a).................. 179

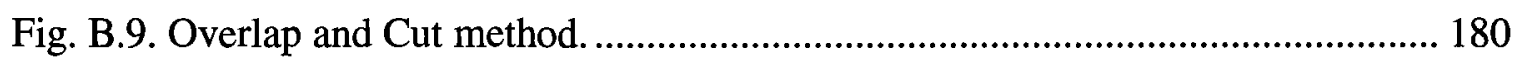

Fig. B.10. SC-FDE-Average BER-Effect of equal power AOCIs' CS, $M=2, K=P=Z=1$

@0dB. 


\section{List of Tables}

\begin{tabular}{|l|l|r|}
\hline Table 2.1. & Average PDP of C2-channel & 20 \\
\hline Table 3.1. & System parameters & 45 \\
\hline Table 4.1. & Modified simulation parameters & 66 \\
\hline Table 4.2. & Simulation scenarios & 66 \\
\hline Table 6.1. & Summary of the comparison between different algorithms & 108 \\
\hline Table 6.2. & Improvement via CE over multiple frames (LS-IS) & 111 \\
\hline Table 7.1. & WINNER-Frame structure, simulation and system parameters & 122 \\
\hline Table 7.2. & Summary of the comparison between different algorithms with TE & 129 \\
\hline Table 7.3. & Complexity analysis & 137 \\
\hline Table 8.1. & B-IFDMA parameters & 145 \\
\hline Table 8.2. & Summary of the comparison between B-IFDMA and L-FDMA- & 157 \\
& $4 \times 3$ (1.25Mbaud) & 158 \\
\hline Table 8.3. & $\begin{array}{l}\text { Summary of the comparison between B-IFDMA and L-FDMA- } \\
4 \times 3 \text { and 8x6 (2.5Mbaud and 5Mbaud) }\end{array}$ \\
\hline
\end{tabular}




\section{Notation}

\section{Definitions:}

UPPER CASE letters

[.]

Lower case letters

(.)

Bold

Scalars
Frequency domain variables.

Frequency domain quantity.

Time domain variables.

Time domain quantity.

\section{Vectors or Matrices.}

Italic.

- $(.)^{*}$ denotes complex conjugate.

- $(.)^{\mathbf{T}}$ denotes transpose.

- $(.)^{\mathrm{H}}$ denotes Hermitian transpose.

- $\mathbf{x}=\left(\mathrm{x}_{1}, \mathrm{x}_{2}, \ldots, \mathrm{x}_{N}\right)$ is a row vector.

- $\mathbf{X}=\left[\begin{array}{ll}X_{11} & X_{12} \\ X_{21} & X_{22}\end{array}\right]$ is a matrix.

- $\operatorname{diag}(\mathbf{x})$ gives a vector containing diagonal values of the matrix $\mathbf{x}$.

- $\mathbf{I}_{X}$ is an $X^{*} X$ identity matrix.

- $P(\mathrm{X})$ represents probability of an event $\mathrm{X}$.

- $\operatorname{Re}\{x\}$ represents real part of the complex number $x$.

- $\operatorname{Im}\{x\}$ represents imaginary part of the complex number $x$.

- $\operatorname{Det}(\mathbf{X})$ is determinant of a matrix $\mathbf{X}$.

- $\operatorname{tr}(\mathbf{X})$ is trace of a matrix $\mathbf{X}$.

- $Q(x)$ is the Gaussian probability integral defined by:

$$
Q(x)=\frac{1}{\sqrt{2 \pi}} \int_{x}^{\infty} e^{-\frac{y^{2}}{2}} d y
$$

- $(.)^{-1} X \quad$ Inverse of a matrix of size $X$. 


\section{List of Acronyms}

\begin{tabular}{|l|l|}
\hline AOCI(s) & Asynchronous OCI(s). \\
\hline Avg. & Average. \\
\hline BER(s) & Bit Error Rate(s). \\
\hline B-IFDMA & Block Interleaved Frequency Division Multiple Access. \\
\hline BPSK & Binary Phase Shift Keying. \\
\hline BS(s) & Base Station(s). \\
\hline CC & Convolutional Code. \\
\hline CDF & Cumulative Distribution Function. \\
\hline CDMA & Code Division Multiple Access. \\
\hline CE & Channel Estimation. \\
\hline CFR(s) & Channel Frequency Response(s). \\
\hline CIR(s) & Channel Impulse Response(s). \\
\hline CLT & Central Limit Theorem. \\
\hline CMA & Constant Modulus Algorithm. \\
\hline CP(s) & Cyclic Prefix(s). \\
\hline CS & Channel Shortening. \\
\hline dB(s) & Decibel(s). \\
\hline DFE(s) & Decision Feedback Equalizer/Equalization(s). \\
\hline DFICE & Decision Feedback ICE. \\
\hline DFT & Discrete Fourier Transform. \\
\hline DMT & Discrete Multi-Tone. \\
\hline FDE & Frequency Domain Equalization. \\
\hline FER & Frame Error Rate. \\
\hline FFT & Fast Fourier Transform. \\
\hline FMT & Frequency Multiplexed Training. \\
\hline FMT-Chu & Frequency Multiplexed Training with Chu sequences. \\
\hline GA & Gaussian Approximation. \\
\hline
\end{tabular}




\begin{tabular}{|l|l|}
\hline GMC & Generalized Multi-Carrier. \\
\hline IBI & Inter-Block Interference. \\
\hline ICE & Iterative Channel Estimation. \\
\hline ICI & Inter-Carrier Interference. \\
\hline ICU(s) & In-Cell User(s). \\
\hline IEEE & International Electrical and Electronics Engineers. \\
\hline IFDMA & Interleaved Frequency Division Multiple Access. \\
\hline IFFT & Inverse Fast Fourier Transform. \\
\hline ISI & Inter-Symbol Interference. \\
\hline km/hr & Kilometer per hour. \\
\hline LE & Linear Equalizer/Equalization. \\
\hline L-FDMA & Local Frequency Division Multiple Access. \\
\hline LI & Linear Interpolation. \\
\hline LMS & Least Mean Square. \\
\hline LOS & Line Of Sight. \\
\hline LP & Low Pass. \\
\hline LS & Least Squares. \\
\hline LS-IS & Least Squares Interference Suppression. \\
\hline MC & Multi-Carrier. \\
\hline MIMO & Multiple-Input Multiple-Output. \\
\hline MLSE & Maximum Likelihood Sequence Estimation. \\
\hline MMSE & Minimum Mean Squared Error. \\
\hline MSE & Mean Squared Error. \\
\hline OCI(s) & Out of Cell Interferer(s). \\
\hline OCU(s) & Out of Cell User(s). \\
\hline OFDM & Orthogonal Frequency Division Multiplexing. \\
\hline PACE & Pilot-Aided Channel Estimation. \\
\hline PAPR & Peak to Average Power Ratio. \\
\hline PCSI & Perfect Channel State Information. \\
\hline PDP & Power Delay Profile. \\
\hline
\end{tabular}




\begin{tabular}{|l|l|}
\hline PIC & Parallel Interference Cancellation. \\
\hline QP & Quarter Power. \\
\hline QPSK & Quadrature Phase Shift Keying. \\
\hline RLS & Recursive Least Squares. \\
\hline Rx & Receiver. \\
\hline SD-IBDFE & Soft Decision-based Iterative Block DFE. \\
\hline SDMA & Space Division Multiple Access. \\
\hline SIC & Successive Interference Cancellation. \\
\hline SINR & Signal to Interference plus Noise Ratio. \\
\hline SISO & Single-Input Single-Output. \\
\hline SNR(s) & Signal to Noise Ratio(s). \\
\hline SoISoO & Soft-Input Soft-Output. \\
\hline SS-IS & Sub-Space Interference Suppression. \\
\hline SoISoO-IBDFE & Soft-Input Soft-Output Iterative Block DFE. \\
\hline STTD & Space Time Transmit Diversity. \\
\hline TASC & Training Aided Single Carrier. \\
\hline TDE & Time Domain Equalizer/Equalization. \\
\hline TE & Turbo Equalizer/Equalization. \\
\hline TMT & Time Multiplexed Training. \\
\hline TMT-Chu & Time Multiplexed Training with overlapping Chu sequences. \\
\hline TMT-QPSK & $\begin{array}{l}\text { Time Multiplexed Training with overlapping random QPSK } \\
\text { sequences. }\end{array}$ \\
\hline WINNER & Wireless World Initiative New Radio. \\
\hline
\end{tabular}




\section{List of Symbols}

\begin{tabular}{|c|c|c|}
\hline Symbol & Explanation & $\begin{array}{c}\text { Page } \\
\text { (where symbol } \\
\text { defined) }\end{array}$ \\
\hline$E_{\text {OCI }}$ & Transmitted OCI symbol energy. & 73 \\
\hline$\Psi^{j}[n]$ & $\begin{array}{l}\text { An estimate of the 'out of cell interference free' array input at } \\
j \text { th DFICE iteration and } n \text {th frequency. }\end{array}$ & 129 \\
\hline$\varepsilon_{\mathrm{OCI}}$ & $\begin{array}{l}\text { Attenuation factor of each interfering OCI due to the applied } \\
\text { propagation model. }\end{array}$ & 73 \\
\hline$\nabla^{j}[n]$ & $\begin{array}{l}\text { Raw estimate of the OCIs plus the additive noise at } j \text { th DFICE } \\
\text { iteration and } n \text {th frequency. }\end{array}$ & 129 \\
\hline$\Phi$ & 2x1D Interpolation filter coefficient. & 32 \\
\hline a & Base Chu sequence of length $B$. & 41 \\
\hline$\dot{\mathbf{a}}$ & Base Chu sequence of length $T_{\mathrm{r}}$. & 41 \\
\hline $\mathbf{F}_{B}$ & $B$-point FFT. & 15 \\
\hline $\mathbf{F}_{B}^{-1}$ & $B$-point IFFT. & 22 \\
\hline$\vec{J}$ & Conditional MSE averaged over ICUs. & 79 \\
\hline $\mathbf{R}_{\mathrm{H}_{\mathrm{p}} \mathrm{H}_{\mathrm{p}}}$ & Auto-correlation matrix used in $2 \times 1 \mathrm{D}$ interpolation. & 34 \\
\hline $\mathbf{R}_{\mathrm{HH}_{\mathrm{p}}}$ & Cross-correlation vector used in $2 \times 1 \mathrm{D}$ interpolation. & 34 \\
\hline$\varsigma_{k}$ & Distance of user $k$ from the desired BS. & 18 \\
\hline$\hat{\mathbf{H}}$ & Estimated channel frequency response matrix. & 23 \\
\hline$\hat{\mathbf{W}}$ & Estimated forward equalizer weight matrix. & 23 \\
\hline$\ddot{\mathrm{A}}$ & $\begin{array}{l}\text { FFT of the vector containing Chu training sequences of all } \\
\text { users. }\end{array}$ & 38 \\
\hline$\tilde{\mathbf{S}}$ & Frequency domain hard decisions of the desired ICUs. & 21 \\
\hline$\hat{\mathbf{S}}$ & Frequency domain soft decisions of the desired ICUs. & 81 \\
\hline $\bar{\mu}$ & $K$-dimensional Lagrange multiplier. & 81 \\
\hline
\end{tabular}




\begin{tabular}{|c|c|c|}
\hline$\vec{z}$ & Prime number relative to $T_{r}$. & 26 \\
\hline$\breve{\mathbf{Y}}$ & Received vector at antenna array during training phase. & 39 \\
\hline$\breve{\mathbf{H}}$ & Short or sparse estimated channel frequency response matrix. & 39 \\
\hline $\mathbf{v}$ & Time domain equalizer output. & 22 \\
\hline$\overline{\sigma_{x}^{2}}$ & Variance of the channel estimation error. & 56 \\
\hline $\mathbf{I}_{K}$ & Identity matrix of size $K$. & 22 \\
\hline $\mathbf{I}_{M}$ & Identity matrix of size $M$. & 22 \\
\hline$x_{m}^{k}[n]$ & $\begin{array}{l}\text { Channel estimation error for the } k \text { th ICU at } m \text { th element and } \\
n \text {th frequency. }\end{array}$ & 51 \\
\hline$\hat{\mathbf{F}}$ & Estimated SD-IBDFE or SoISoO-IBDFE feedback coefficient. & 81 \\
\hline $\boldsymbol{\Omega}$ & $\begin{array}{l}\text { Matrix of the conditional MSE difference between data } \\
\text { symbols and their soft decisions. }\end{array}$ & 80 \\
\hline$\hat{\Gamma}[n]$ & Time averaged correlation matrix at frequency $n$. & 129 \\
\hline$\overline{\sigma_{\mathrm{OCI}}^{2}}$ & Variance of the inter-cell interference noise term. & 73 \\
\hline $\mathbf{\Omega}_{v}$ & $\begin{array}{l}\text { Vector of the conditional MSE difference between data } \\
\text { symbols and their soft decisions. }\end{array}$ & 80 \\
\hline$\lambda$ & Reliability threshold in DFICE. & 90 \\
\hline$\Omega$ & Path loss exponent. & 18 \\
\hline$\sigma_{n}^{2}$ & Noise variance. & 16 \\
\hline $\bar{B}$ & Total usable sub-carriers. & 15 \\
\hline$D_{n}$ & Pilot-spacing in frequency direction. & 29 \\
\hline$d_{\mathrm{OCI}}$ & Relative delay of the AOCIs. & 168 \\
\hline$D_{q}$ & Pilot-spacing in time direction. & 29 \\
\hline$F$ & Total number of SC-FDE blocks per frame. & 19 \\
\hline$f_{\text {Dmax }}$ & Maximum Doppler frequency. & 29 \\
\hline$F_{\mathrm{r}}$ & $\begin{array}{l}\text { Total number of frames used in CE (time domain interpolation, } \\
\text { LS) processing. }\end{array}$ & 108 \\
\hline $\mathbf{H}$ & Channel frequency response matrix. & 16 \\
\hline$I_{\mathrm{E}}$ & Total number of SD-IBDFE iterations. & 82 \\
\hline
\end{tabular}




\begin{tabular}{|c|c|c|}
\hline$I_{\text {ICE }}$ & Total number of DFICE iterations. & 89 \\
\hline$I_{r}$ & Cutoff of the LP smoothing filter. & 29 \\
\hline$J_{0}$ & Zeroth order Bessel's function of first kind. & 35 \\
\hline$J_{\text {est }}$ & Mean Squared Error (MSE) at the equalizer output with CE. & 52 \\
\hline$J_{\min }$ & $\begin{array}{l}\text { Minimum Mean Squared Error (MMSE) at the equalizer } \\
\text { output. }\end{array}$ & 22 \\
\hline$K$ & Total number of In-Cell Users (ICUs). & 14 \\
\hline$L$ & Channel memory. & 15 \\
\hline$L_{\mathrm{CP}}$ & Length of the Cyclic Prefix. & 168 \\
\hline$M$ & Total number of receiver (BS) antenna elements. & 14 \\
\hline$N T$ & $\begin{array}{l}\text { Total number of short training blocks in Time Multiplexed } \\
\text { Training (TMT). }\end{array}$ & 38 \\
\hline$P$ & Total number of Out of Cell Interferers (OCIs). & 60 \\
\hline$Q_{n}$ & $\begin{array}{l}\text { Number of available pilots in the frequency axis interpolation } \\
\text { sliding window. }\end{array}$ & 32 \\
\hline$Q_{q}$ & $\begin{array}{l}\text { Number of available pilots in the time axis interpolation sliding } \\
\text { window. }\end{array}$ & 32 \\
\hline $\mathbf{S}$ & Frequency domain data symbols. & 17 \\
\hline$T$ & Symbol period. & 15 \\
\hline$T_{r}$ & $\begin{array}{l}\text { Length of the short training block or number of pilots in a } \\
\text { block. }\end{array}$ & 24 \\
\hline $\mathbf{V}$ & Frequency domain equalizer output. & 22 \\
\hline $\mathbf{W}$ & Forward equalizer coefficients with PCSI. & 22 \\
\hline$X$ & Total number of sub-carriers (FFT size). & 15 \\
\hline $\mathbf{Y}$ & Array input frequency domain vector. & 16 \\
\hline $\mathbf{y}$ & Array input time domain vector. & 15 \\
\hline$Z$ & Total number of Asynchronous Out of Cell Interferes (AOCIs). & 168 \\
\hline
\end{tabular}




\section{Chapter 1}

\section{Introduction}

\subsection{Thesis Objectives}

Next generation wireless communication is required to provide high-speed, high-quality multimedia transmission. However, there exist challenges with frequency selective channels, which are introduced by the multi-path effect. Frequency-Domain Equalization (FDE) has been shown to be an attractive solution for frequency selective channels [FAB02]. FDE outperforms the conventional Time-Domain Equalization (TDE), as it requires less computational complexity by using Fast Fourier Transform (FFT). Orthogonal Frequency Division Multiplexing (OFDM) and Single Carrier with Frequency Domain Equalization (SC-FDE) are the two fundamental FDE techniques. Compared to OFDM, single carrier has lower Peak-to-Average Power Ratio (PAPR) and is less sensitive to carrier synchronization. Therefore, SC-FDE is a strong candidate for the uplink (mobile user to Base Station (BS) transmission) scenario in order to keep the cost and complexity of the user terminal low. The objective of this thesis is to study the multi-user Channel Estimation (CE) for SC-FDE uplink systems.

In an uplink SDMA cellular system, the BS has to deal with inter-user interference along with the Inter Symbol Interference (ISI). Inter-user interference can arise with two kinds of users: the In-Cell co-channel Users (ICUs) and users from adjacent or neighboring cells. We call the latter Out of Cell Interferers (OCIs). The interference scenario considered in this thesis is depicted in Figure 1.1 where the BS of the desired cell (the cell in the middle) is experiencing in-cell as well as out of cell interferences while estimating the channels of one of the ICU. 


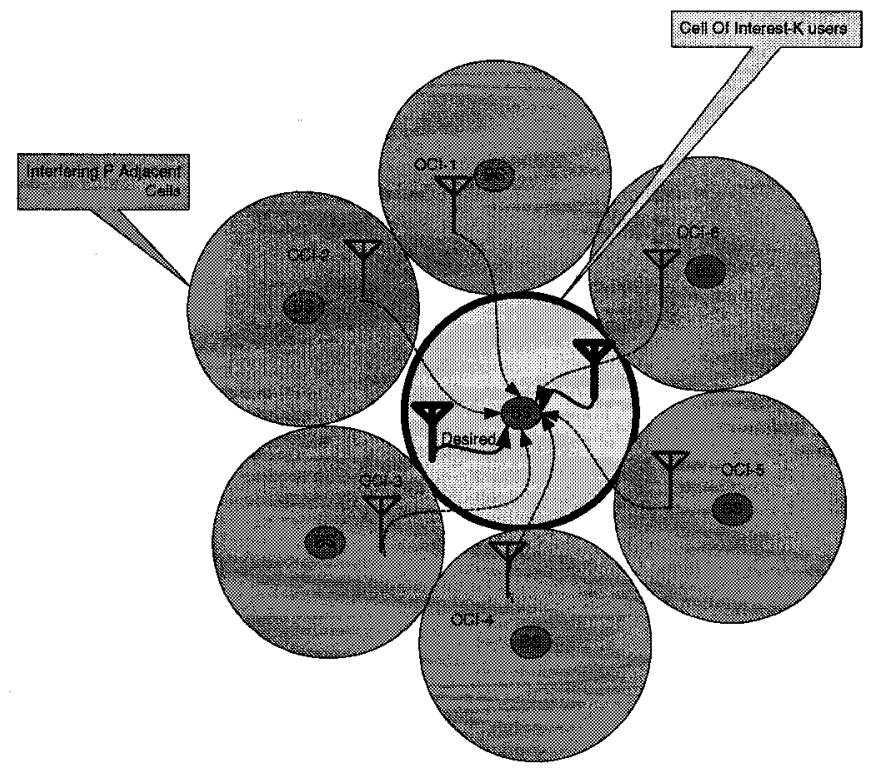

Fig. 1.1. Distribution of intra and inter-cell interference.

Usually, all the ICUs in a given cell are of interest and their channels are to be estimated for equalization purposes. All the ICUs cause intra-cell interference. Also co-channel users in the neighboring cells cause inter-cell interference. The objective of this thesis is to explore the receiver processing in order to detect the data and estimate the channels of the desired users in such an interference-prone SC-FDE uplink environment.

There are three main techniques used to improve any communication link:

1. Equalization

2. Diversity

3. Channel coding

We will use different forms of these three techniques for improving the multi-user detection and $\mathrm{CE}$ process. In interference-prone systems, good channel estimation is possible only if we can suppress the inter-user interference. There are three major approaches to deal with the interference. First, interference averaging, second interference avoidance and third is interference suppression via smart antennas. In this thesis we consider the second and third approach of dealing the interference suppression to improve the desired users' channel estimates.

First we apply interference avoidance techniques. In-cell interference avoidance is done by appropriate design of the transmitted signal structure, use of optimum training 
sequences and their coordinated placements. Multi-cellular interference avoidance includes some kind of upper layer co-ordination to keep the average received power of the co-channel OCIs low. Such control can be achieved by appropriate frequency reuse strategy, sectorization and spread spectrum. After achieving the desired advantages provided by the avoidance methods we apply interference rejection via signal processing at the receiver. Interference suppression via receiver signal processing involves smart antennas, soft decision-based iterative equalization and turbo processing. After suppressing the interference we perform channel estimation that, utilizes the interferencesuppressed signal in hard and soft decision feedback-based iterative channel estimation. The CE aspects of a SC-FDE-based system in the presence of ICUs and OCIs are relatively new and not yet explored much. Smart antenna techniques have been shown to be effective in reducing co-channel interference at the receiver side, when supporting multiple users. In this work, we investigate array signal processing as an Interference Rejection Combining (IRC) technique combined with some novel equalization and adaptation algorithms to update the channel estimates with iterative processing. Improved hard or soft decisions are used in the proposed Decision Feedback Iterative Channel Estimation (DFICE) algorithm. In summary, the focus of this thesis is to develop and investigate the design and performance of different $\mathrm{CE}$, equalization and adaptation techniques with improved performance obtained via a combination of orthogonal training, spatial diversity and iterative receiver processing.

\subsection{Thesis Contributions}

This thesis proposes techniques to combat multi-user interference in SC-FDE blockbased communication system under a time varying frequency selective fading environment. The following are the contributions:

- We investigate the use of different interpolation techniques and optimum training sequences to obtain good pilot-assisted initial multi-user channel estimates. With SC-FDE, multi-user uplink scenario, we introduce two ways of multiplexing the pilots with the data: Time Multiplexed Training (TMT) and Frequency Multiplexed Training (FMT). It is shown that an arrangement of frequency- 
multiplexed pilots has the same performance as an equivalent conventional arrangement of the time-multiplexed pilots. Different pilot placements among different In-Cell Users (ICUs) are compared, showing that the channel estimates in the presence of interference can be improved by keeping their training signals or pilots orthogonal to one another. This requires time and frequency resources proportional to the number of interferers. (Contribution appears in Chapter 2 and 3)

- To deal with the Out of Cell Interferers (OCIs) different types of compensations and pilot placements are introduced. One approach would be to keep the OCIs' pilots orthogonal to the pilots of ICUs. Orthogonal OCIs' pilots require that each cell leave further unused time or frequency resources for adjacent interfering cells' pilots. Known OCI's training could further help to improve their estimates. This requires high overhead; it is shown that with low power OCIs, overlapping pilots become more practical and with the help of spatial processing a reasonable performance is achievable with overlapping pilots. But in the case of equal power ICUs' overlapping pilots, the channel estimates deteriorate significantly. We suggest keeping the ICUs' pilot orthogonal to each other while allowing the OCIs' pilots to overlap. All cells thus share the same time and frequency resources for their pilots. It is observed that although the strength of OCIs could be very weak, their number could be large enough to deteriorate the In-cell CE performance. (Contribution appears in Chapter 4)

- We also study the impact of multi-user channel estimation errors on the Mean Squared Error (MSE) of the equalizer output with or without unknown OCIs. (Contribution appears in Chapter 3 and 4)

- A novel low complexity multi-user Soft Decision-based Iterative Block Decision Feedback Equalizer (SD-IBDFE) is introduced which uses soft decisions to adapt the antenna array feed forward and feedback coefficients and suppresses the ISI and the Inter-user interference. Such structures reduce the complexity of the nonlinear processing compared to the time domain approaches. Also as the feedback loop takes into account not just the decisions for each block but also the overall 
block reliability, error propagation is reduced. (Contribution appears in Chapter 5)

- We have developed a structure of a low-complexity hard decision-based multiuser DFICE (HD-DFICE). The threshold-based structure of the hard decisionbased multi-user DFICE utilizes the coding gain and feeds the SD-IBDFE with multi-user's frequency domain channel estimates relatively better than the solely pilot-based estimates. In SC-FDE, the envelope of the frequency response of the decisions is not constant which creates noise enhancement at certain frequencies while computing the CFR via decisions. A threshold-based solution is introduced in which the CFR obtained from decisions at noise enhancement frequencies are replaced by the CFR estimates of the previous ICE iteration. We name this Frequency Replacement Technique (FRT). An Expectation Maximization (EM) algorithm-based rationale is presented to justify the use of FRT algorithm. It addresses and solves the problem of noise enhancement in SC-FDE DFICE channel estimates due to data sequence frequency selectivity. We also show that an iterative receiver impressively increases the number of supported users in an interference-prone environment when Parallel Interference Cancellation (PIC) supports the proposed HD-DFICE. We propose a Least Square (LS) adaptation in combination of PIC assisted SD-IBDFE, which can suppress the interference caused by In-Cell and Out of Cell users without explicitly estimating the channel links of the OCIs. (Contribution appears in Chapter 6)

- We present a Low-Density Parity-Check (LDPC) code-based Turbo Equalization (TE), which improves the equalization and DFICE performance. It works in combination with LS interference suppression, PIC and Soft-In Soft Out (SoISoO)-IBDFE algorithms, and shows gain in terms of the required Signal-toNoise Ratio (SNR) to achieve a certain Frame Error Rate (FER). In the presence of Out of Cell Interference a further channel estimation improvement is achieved by using a sub-space-based interference cancellation method. The threshold-based structure of the multi-user HD-DFICE is altered and by using an EM algorithm approach a Soft Decisions-based DFICE (SD-DFICE) algorithm is proposed. (Contribution appears in Chapter 7) 
- CE aspects of a newly introduced chunk-based multiple access schemes in the WINNER $^{2}$ concept, named Block-Interleaved Frequency Division Multiple Access (B-IFDMA) and Local-IFDMA (L-IFDMA) are explored. Pilot design for multi-user transmission in chunk-based systems is proposed in order to reduce the transmission overhead. HD-DFICE proposed for the full bandwidth systems is applied to these partial band systems. (Contribution appears in Chapter 8)

- We also show that a multi-frame multi-user CE strategy in both full bandwidth and chunk-based systems is useful and provide some additional gain at slightly higher complexity. (Contribution appears in Chapter 6 and 8 )

- Finally, we present methods like time domain processing and overlap-cut method to deal with the asynchronous OCIs. (Contribution appears in Appendix B)

Publications referenced as [SDF07a], [SDF07b], [SDF08a], [SDF08b] and [SDF08c] are based on the contributions described above.

\subsection{Thesis Structure}

Chapter 2 describes some of the background topics like signal and channel model to be used in the rest of the thesis and the basic techniques of multi-user $\mathrm{CE}$ and equalization. Chapter 3 and 4 describes the $\mathrm{CE}$ and interference suppression by using different interference avoidance techniques like pilot placements in the presence of intra and intercell interference. Chapter 5, 6 and 7 explicitly describe the proposed CE and interference suppression algorithms. Chapter 8 is dedicated to the CE and interference suppression in chunk-based systems. Chapter 9 describes conclusions and possible future research work.

Detailed chapter wise organization of the thesis is as follows.

Chapter 2 presents an overall multi-user Single Carrier Frequency Domain Equalization (SC-FDE) signal, channel and system model. A Generalized Multi-Carrier (GMC) SDMA transceiver structure is presented. This chapter also provides necessary

\footnotetext{
${ }^{2}$ WINNER-Wireless World Initiative New Radio is a consortium of 41 members coordinated by Nokia-Siemens Networks. https://www.ist-winner.org/
} 
background and related literature review on channel estimation, optimum pilot design, pilot multiplexing, interpolation techniques, channel equalization, adaptation techniques and previous work done towards SC-FDE MIMO equalization and CE.

Chapter 3 deals with the uplink pilot-based adaptive linear receiver spatial-frequency processing techniques for efficiently mitigating the intra-cell interference. Our focus in this chapter is on pilot-assisted CE techniques and on exploring different ways of intracell interference suppression via pilot placement. Efficient inter-user pilot coordination is proposed. Comparison between orthogonal and overlapping pilots, performance of different interpolation techniques and their complexities and the effect of mobility on the pilot multiplexing are illustrated via simulations. Finally, BER and MSE analysis with Perfect Channel State Information (PCSI) and with CE is presented.

Chapter 4 introduces the effects of the co-channel Out of Cell Interferers (OCIs) (Intercell Interference) on the multi-user CE process. These OCIs can have known or unknown training sequences, and hence affect the CE performance accordingly. This chapter proposes different forms of compensations and training arrangements that can be used to suppress the effects of OCIs. Simulation results compare the gain obtained from receiver diversity, knowing the training sequences of the OCIs and orthogonal placement of different users in the system. A Gaussian Approximation (GA) based OCI modeling is also presented.

Chapter 5 describes the structure of a proposed low complexity Soft-Decision-based Iterative Block Decision Feedback Equalizer (SD-IBDFE). The proposed SD-IBDFE helps in efficiently suppressing the inter-user interference along with Inter Symbol Interference (ISI). The performance gain via iterative equalization is illustrated through computer simulations. It is observed that additional diversity gain improves the SDIBDFE performance and reduces the SNR degradation. This gives a notion that with improved channel estimates via any decision-directed approach, the proposed SD-IBDFE can efficiently combat the interference. This is verified in Chapter 6 when we use the SDIBDFE in combination with different proposed DFICE algorithms. Moreover, as SD- 
IBDFE computes and updates the soft decisions, it is modified in Chapter 7 to accommodate the Turbo Equalization (TE).

Chapter 6 proposes three extensions to the multi-user CE problem. A Decision Feedback Iterative CE (DFICE) technique is introduced. The proposed DFICE utilizes the gain from the SD-IBDFE to compute the hard decisions and feeds back the updated channel estimates to the SD-IBDFE iteratively. Parallel Interference Cancellation (PIC)-supported DFICE is the second step introduced in the chapter to further improve the DFICE performance. PIC removes the effects of the intra-cell interference and achieves a cleaned version of the array input to be used in DFICE. The last section of the chapter is dedicated to the proposed Least Squares (LS) interference suppression algorithm. In this algorithm, a LS forward filter processing is used to further mitigate the inter-user interference.

Chapter 7 describes the Turbo Equalization (TE) assisted CE techniques. A Low-Density Parity-Check (LDPC) code based TE is used. An improved Soft Decisions-based DFICE (SD-DFICE) is derived from an EM algorithm. The proposed interference cancellation scheme with forward LS processing described in Chapter 6 is combined with LDPC codebased TE. SD-DFICE performance is improved because of the soft information transfer between the equalizer and the decoder. Moreover, a Sub-Space (SS) based method is also introduced to suppress the OCIs explicitly.

Chapter 8 is dedicated to the CE aspects for the chunk-based or split-spectrum system with SC-FDE transmission. In this chapter, the multi-user SD-IBDFE and proposed DFICE is applied to suppress the intra-cell and inter-cell interference in a chunk-based system. A chunk-based system introduced under the WINNER concept, named Block Interleaved Frequency Division Multiple Access (B-IFDMA), is described. Performance of the CE with orthogonal ICUs' pilots and overlapping OCIs is evaluated under different pilot overheads. For comparison purposes the B-IFDMA is compared with the Full Bandwidth (FBW) system as well as with so-called partial-band Local Frequency Division Multiple Access (L-FMDA) system. 
Finally, chapter 9 describes conclusions and possible future research directions. 


\section{Chapter 2}

\section{Frequency Domain Processing in Uplink SDMA Cellular Systems}

\subsection{Introduction}

In this chapter, we describe the preliminaries of the thesis with some general background and previous work related to the multi-user Single Carrier with Frequency Domain Equalization (SC-FDE) systems and initial Channel Estimation (CE). Sections 2.2, 2.3 and 2.4 describe an overview of multi-user SC-FDE approach with a brief description of some initial work done on SC-FDE. A Space Division Multiple Access (SDMA) SC-FDE signal and channel model defined in WINNER [Win01] concept is described in Section 2.5. Some key concepts involved in SDMA Linear Equalization (LE) and array adaptation techniques are briefly stated in Section 2.6. Section 2.7 describes a brief overview of the optimum initial pilot-aided CE including an introduction of pilot design, pilot multiplexing, interpolation and time domain smoothing filter.

\subsection{Single Carrier Frequency Domain Equalization (SC-FDE)}

Block-based transmission with Frequency Domain Equalization (FDE) has been shown to be an attractive technique for frequency selective channels [FAB02], [SKJ95]. Compared to the Time-Domain Equalization (TDE), FDE outperforms in complexity with the use of Fast Fourier Transform (FFT). The hardware complexity with FDE is approximately proportional to the logarithm of the maximum delay spread of the channel [FAB02]. There are mainly two frequency domain approaches that counteract the Inter-Symbol Interference (ISI) phenomenon in a distinct manner: OFDM (Orthogonal Frequency Division Multiplexing) and SC-FDE (Single-Carrier with Frequency Domain Equalization). 
SC-FDE is an approach that counteracts the Peak to Average Power Ratio (PAPR) problem associated with OFDM while keeping the same low complexity of the whole equalization process, similar to that of the OFDM. The data is transmitted using a single carrier with a classical modulation scheme, which eliminates the problem of expensive power amplifiers to handle a high PAPR. The whole complexity of the equalization is distributed at the receiver and is still based on FFT processing. Better power amplifier efficiency (requiring smaller power back-off) is the reason SC-FDE is proposed for uplink communication [FAB02] and [DGE04 and references therein]. A simplified block diagram of a coded SC-FDE transceiver is shown in Figure 2.1.

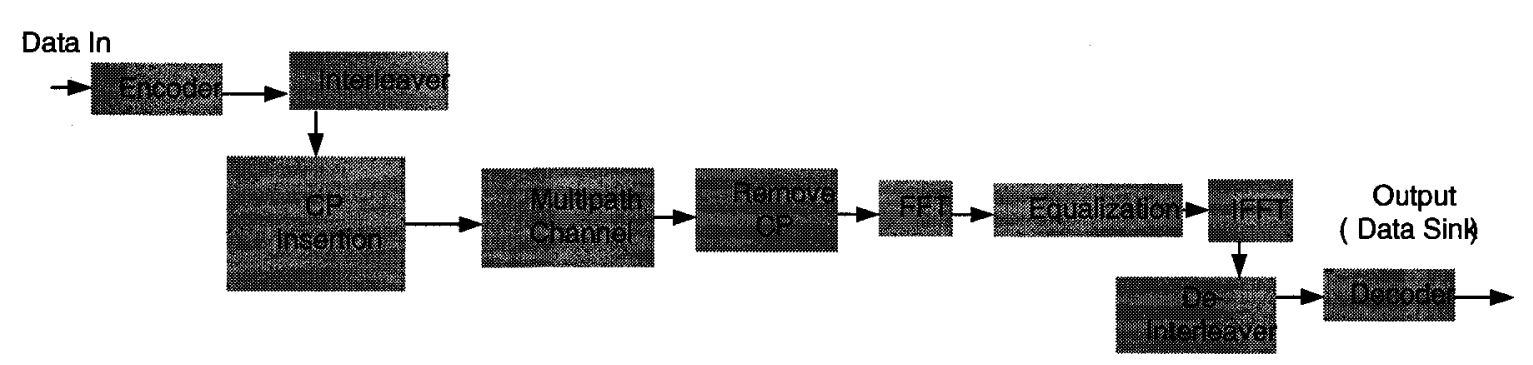

Fig. 2.1. Block diagram of a coded SC-FDE transceiver.

\subsubsection{Initial Work on SC-FDE}

SC-FDE is relatively new in wireless communications. It was first introduced in [WS73] in the early 1970s and afterward in [Fer85]. A detailed introductory description of the SC-FDE and its comparisons with OFDM, equalization issues, analysis with known channels and $\mathrm{CE}$ is given in [FAB02]. Eventually a mixed mode is defined in which a coexistence of OFDM and SC-FDE is proposed that is OFDM in downlink and terminals use SC-FDE for uplink communication.

Kadel introduced a receiver structure based on the SC-FDE in [Kad97]. [Czy97] compared single carrier systems with OFDM employing Fast Fourier Transform (FFT). Volker compared SC-FDE with OFDM in quasi-static channel conditions with random coding techniques [AFV98]. They showed that both systems perform differently at different code rates. They advocated adopting the Minimum Mean Squared Error (MMSE) criteria for updating the Linear Equalizer (LE) coefficients. The initial work on 
pilot-assisted synchronization in SC-FDE is done in [Czy98]. In [GDC00], SC-FDE is compared with a general Multi-Carrier (MC) system in terms of FDE, impact of channel coding and power amplifier requirements with back-off issues. [TCP01] also compared OFDM and SC-FDE in terms of phase noise and power amplifier performance, and showed that OFDM and SC-FDE have the same phase-noise sensitivity provided the number of used sub-carriers is not large. It is shown in [PVM95] that the performance degradation due to phase noise in OFDM is proportional to the number of sub-carriers while in SC-FDE the expression for the Signal to Noise Ratio (SNR) degradation due to phase noise is independent of the number of sub-carriers. However, it is shown in [TCP01] that SC-FDE has increased power amplifier efficiency and therefore a good choice for the portable high data rate terminals. Noise variance estimation in SC-FDE is addressed in [CSB04]. It was shown that a good quality channel estimates are necessary to reliably estimate the variance of the additive Gaussian noise.

\subsection{MIMO SC-FDE}

A single transmitting source with multiple antennas, multiple desired users in a system or presence of several interferers all constitute a MIMO channel. Our objective is to design and evaluate FDE and CE algorithms in such channel conditions. In particular, we will mainly concentrate on the uplink (terminal to Base Station (BS)) of a cellular system employing a SDMA scheme.

Initial work towards MIMO-SC-FDE started with simple transmit diversity schemes which were incorporated with perfect CE in SC-FDE. [WSW99] and [Ald01] proposed the Alamouti space-time coding and also added the receiver diversity in a multi-path fading environment. A comprehensive comparison between MIMO OFDM and MIMO SC-FDE is presented in [CSB03]. Later, a similar work on space-time Transmit Diversity (STTD) is explored in [TIA04]. A series of papers, [KDF04a] and [KDF04b], show the performance of the iterative frequency domain layered space-time receiver structure for MIMO-SC-FDE systems. Capacity improvements and successive as well as Parallel Interference Cancellation (PIC) are the main issues of that work. 


\subsection{Multi-User Channel Estimation}

Our main focus in this thesis is on the multi-user CE aspects in an interference-prone SCFDE multi-cellular system. When addressing intra-cell interference or SDMA users in a cell, all the In-Cell Users (ICUs) are of interest. The CE between each transmitting and receiving antenna pair has to be performed before data demodulation can proceed. The accuracy of the channel estimates is crucial to the performance of the overall system in terms of the Bit Error Rate (BER). However, the quality of the channel estimates depends on how effectively we can estimate and separate multi-user channels from the composite observation. The use of SDMA and aggressive frequency reuse strategies give rise to high levels of co-channel interference, which must be mitigated by spatial processing at the receiver. Spatial processing that adapts to the desired signals', interferers' locations and spatial signatures requires accurate $\mathrm{CE}$ and appropriate allocation of pilot signals. Also the frequency domain array coefficients (function of the estimated Channel Frequency Response (CFR)) given in (2.11) need reliable channel estimates at the receiver. $\mathrm{CE}$ techniques proposed in the literature can be categorized as:

1. Pilot-assisted.

2. Blind.

3. Decision-Feedback.

The pilot or training-based CE is based on the training data sent from the transmitter and known a-priori at the receiver. On the other hand, blind CE exploits the statistical information of the channel and certain properties of the transmitted signals. Though the latter has its advantage in that it has no overhead loss, it is only applicable to slowly time-varying channels. This thesis utilizes the training-based CE. We will also use the iterative receiver processing in which Decision Feedback Iterative CE (DFICE) is employed.

\subsubsection{Previous Work on MIMO Channel Estimation}

In this section we briefly explore some of the previous work related to the CE of multiuser or MIMO channels. Similar to OFDM systems, training sequences or pilots are used to get the initial estimates of the multi-user Channel Frequency Responses (CFRs) in SCFDE systems. Before the advent of the Generalized Multi-Carrier (GMC) [Win02] 
approach of generating SC-FDE signals at the transmitter described in Section 2.5.1, it was assumed that time domain multiplexing is the only way in single carrier systems to multiplex the training with the data [Czy98] and [DGE01]. With the GMC transceiver structure, it is possible in single carrier modulation to insert the training symbols into the data block in a frequency-multiplexed manner. With frequency multiplexed pilots the initial CE schemes employed in OFDM can readily be used in the SC-FDE systems. A comprehensive description on CE in MIMO-OFDM systems is given in [HK06]. With OFDM, the pilot-assisted CE techniques are also investigated in [CS02], [Hoh91, HKR97, IKY98, Li00, TM97, WL00] and [YLC99, YCL01a, YCL01b]. [JTN04] presents the pilot-assisted $\mathrm{CE}$ in the presence of interference with OFDM system. [LSA99] introduces the LS-based DFICE, which makes use of the cross-correlation properties of the transmitted sub-carrier symbol sequences. Optimum training sequences are also proposed in [LSA99]. A reduced complexity version of the LS-based DFICE of [LSA99] is introduced in [MKB02], based on exploiting the channel's correlation in the frequency-direction, as opposed to the channel's correlation in time-direction presented in [Li02]. A similar approach is suggested by [Sli02] with two transmit antennas. Time domain PIC-assisted DFICE is investigated in [Li00b] which is a simplified version of the [LSA99]. A more detailed discussion on the time domain based PIC-assisted DFICE is provided in [Li02]. Frequency domain PIC-based DFICE is studied in [JPC00], which exploits the channel's slow time variation. Blind $\mathrm{CE}$ and equalization using second-order cyclo-stationary statistics as well as antenna precoding techniques are explored in [BHP02]. MMSE-assisted DFICE is presented in [GL01]. Expectation Maximization (EM) based CFR estimation approach for DFICE is presented in [XG01]. Robust subspace based weight vector calculations and tracking are employed for co-channel interference suppression in [WP98].

With SC-FDE [DGE01] proposed transformation of the Cyclic Prefix (CP) into training sequence and named it as Training Aided Single Carrier (TASC). There are a few others who showed the utility of replacing $\mathrm{CP}$ with such a known sequence to be used for different purposes like synchronization and CE. A more common name given is Unique Word (UW) [Witsc02] and [FAB02]. Some later work like [CBM04a] uses UW as a standard part of the SC-FDE system. More recently, [CS05] showed how a well-designed 
UW with good spectral and correlation properties might improve the estimates of the channel. MIMO CE in SC-FDE is elaborated in [SCP04]. A comparison of different SDMA detection techniques is presented in [VPE99], namely MMSE, Successive Interference Cancellation (SIC) and Maximum Likelihood (ML). Further improvements of SIC detection are suggested by adaptively tracking multiple symbol decisions. There is an amount of work being done with OFDM transmission in the context of suppressing cochannel interference in cellular communication systems. [LS98, LS99, LCS98, KCC99] and [MKH99] have explored techniques and algorithms designed for multi-user CE and interference suppression.

Our focus in this thesis will be on CE aspects of a SC-FDE-based system in the presence of In-Cell co-channel Users (ICUs) and Out of Cell Interferers (OCIs), which are not much investigated yet.

\subsection{SC-FDE SDMA Signal and Channel Model}

In this section we describe a general multi-user signal model to be used in the system evaluation via simulations in this thesis. We consider a coded block-based single carrier, SDMA uplink system with $K$ co-channel In-Cell Users (ICUs) and $M$ receiving antenna elements at the desired Base Station (BS). Each ICU has a single antenna element in order to keep the cost of the user terminal low. Linear or iterative equalization, explained in the later chapters, are used to equalize the channel. In this thesis a convolutional encoder/Viterbi decoder is used. An LDPC encoder/decoder is used in the case of Turbo Equalization (TE) (described in Chapter 7). In each frame all ICUs can simultaneously transmit QPSK modulated signals to the BS. At the BS, processing the signal vectors received at the array separates streams of the ICUs.

\subsubsection{Generalized Multi-Carrier (GMC) SDMA Transceiver Structure}

We employed a Generalized Multi-Carrier (GMC) transceiver structure [Win02] for the single carrier multi-user system. The GMC transceiver structure provides a framework for future-generation air interfaces. With GMC transmitters, a more general class of signals such as Direct Sequence-Code Division Multiple Access (DS-CDMA), MultiCarrier CDMA (MC-CDMA) etc. can be generated by either including or excluding the 
FFT matrix. The GMC structure provides a low complexity solution of frequency domain processing in which computational complexity is proportional to the logarithm of the maximum Channel Impulse Response (CIR) length [Taf06]. In this thesis, we will be using the GMC structure when we multiplex pilot symbols with data in frequency domain. A generalized transceiver block diagram is given in Figure 2.2.

Let the $k$ th user group its coded data in blocks of length $B$. Let $s^{k}(q)$ denotes the $q$ th element in a block where $q=0,1 \ldots, B$-1. A $B$-point FFT transforms the block into the frequency domain. Then $X-B$ zeros are padded, and the resulting $X$ samples go through an Inverse FFT (IFFT), followed by the Cyclic Prefix (CP). A CP is added to change the convolution from linear to circular, which allows the efficient frequency domain equalization. The $\mathrm{CP}$ is also used to prevent the Inter-Block Interference (IBI) and to make the received block appear to be periodic with period $X$ [BT02b]. The transmission is through a frequency selective, time-varying channel model with memory $L$. The channel model and the corresponding average Power Delay Profile (PDP) are given in Section 2.5.2 and Table 2.1 respectively. Because of the channel memory, each block is preceded with a $\mathrm{CP}$, at least equal to length $L$, which is the repetition of the last $L$ symbols of the block.

In the frequency domain, the single carrier signal ${ }^{3}$ is the FFT of the $s^{k}(q)$

$$
S^{k}[n]=\sum_{q=0}^{B-1} s^{k}(q) \exp (-j 2 \pi q n / B) \stackrel{\Delta}{=} \mathbf{F}_{B}\left(s^{k}(q)\right),
$$

where $\mathbf{F}_{B}$ represents a $B$-point FFT, $j$ represents $\sqrt{-1}$ and $q$ is the discrete time index for $q=0,1, \ldots, B-1$. Each user's data is assumed to be uncorrelated with that of the others, with unit average symbol energy and symbol period $T$. The frequency domain sequences from the $K$ users are transformed to the time domain by Inverse FFTs (IFFTs) and transmitted through the $M \mathrm{x} K$ independent Channel Impulse Responses (CIRs) $h_{m}{ }^{k}\left(q_{1}\right)$, where $h_{m}{ }^{k}\left(q_{1}\right)$ is the baseband representation of the channel from user $k$ to antenna $m$ as shown in Figure 2.2, for $k=1,2, \ldots, K, m=1,2, \ldots, M$ and $q_{1}=0,1, \ldots, X-1$. At the receiver, the $m$ th antenna element receives the convolutional output, which can be shown to be given by:

${ }^{3}$ Also called DFT-precoded OFDM in the GMC context [Win02]. 


$$
y_{m}\left(q_{1}\right)=\sum_{k=1}^{K} \sum_{q=0}^{B-1} s^{k}(q) b_{m i}^{k}\left(q_{1}-q \frac{X}{B}\right)+n_{m}\left(q_{1}\right)
$$

where $n_{m}\left(q_{1}\right)$ is white Gaussian noise with variance $\sigma_{n}^{2}, s^{k}(q)$ represents the data samples of $k$ th user, and $h_{m}{ }^{k}\left(q_{1}\right)$ represents the oversampled cyclic impulse response of the channel such as $b_{m}^{k}\left(q_{1}\right)=b_{m}^{k}\left(q_{1}+I X\right)$, for any integer $l$. At the receiver we assume that all users are symbol synchronized (the effect of asynchronism is evaluated in Appendix B).

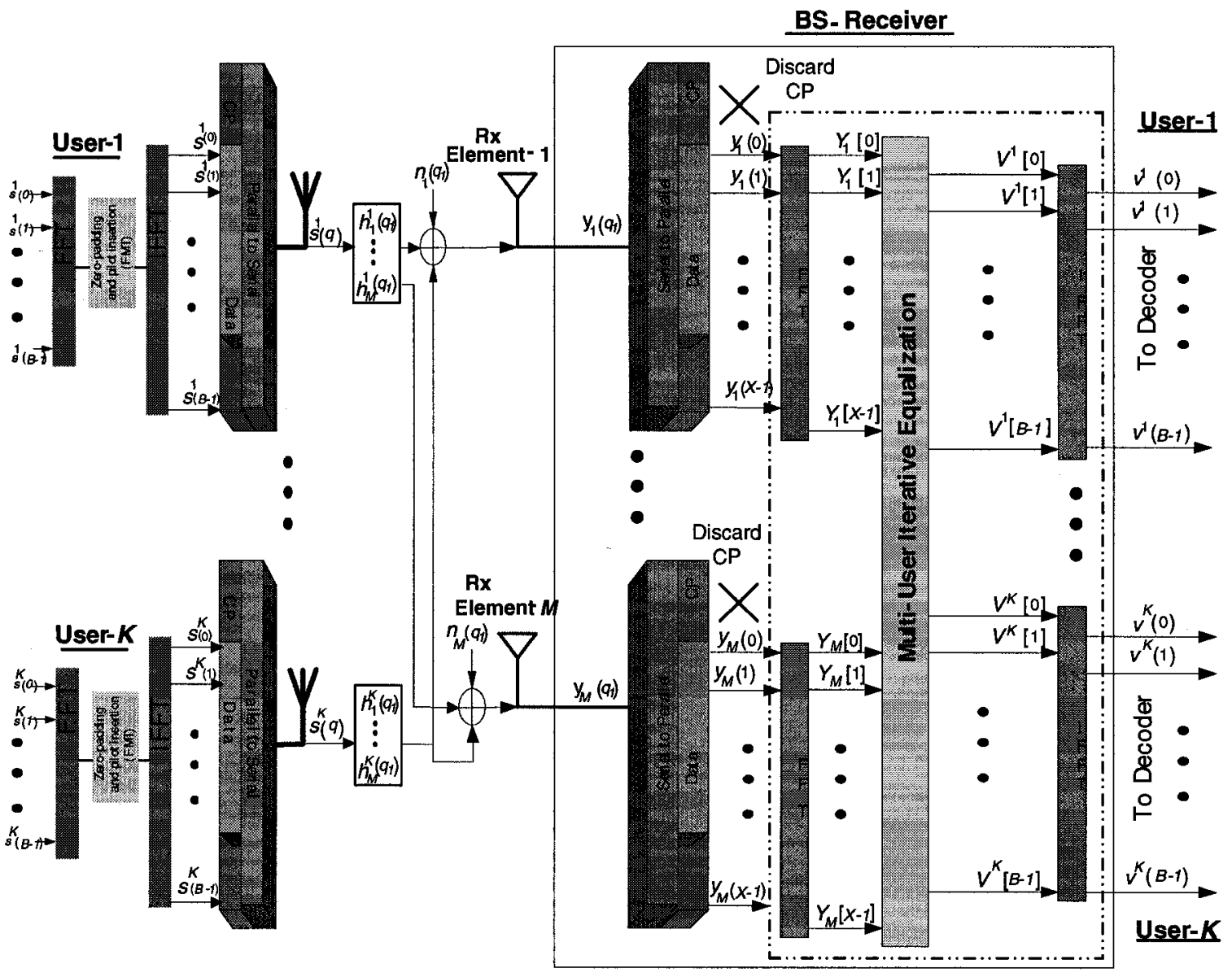

Fig. 2.2. Block diagram of a GMC structure-based SC-FDE transceiver.

After a $X$-point FFT, which yields the $M$ received frequency domain sequences, $\mathrm{Y}\left[n_{1}\right]=\left[\mathrm{Y}_{1}\left[n_{1}\right], \mathrm{Y}_{2}\left[n_{1}\right], \ldots, \mathrm{Y}_{M}\left[n_{1}\right]\right]^{\mathrm{T}}$, where $n_{1}=0,1, \ldots, X-1$, and $n_{1}$ is the sub-carrier index. The cyclic convolution is equivalent to the multiplication in the frequency domain, i.e., we obtain

$$
\mathbf{Y}\left[n_{1}\right]=\mathbf{H}\left[n_{1}\right] \mathbf{S}\left[n_{1}\right]+\mathbf{N}\left[n_{1}\right]
$$


with $\mathbf{Y}\left[n_{1}\right] \in C^{M \times 1}, \mathbf{H}\left[n_{1}\right] \in C^{M \times K}, \mathbf{S}\left[n_{1}\right] \in C^{K \times 1}, \mathbf{N}\left[n_{1}\right] \in C^{M \times 1}$, where $C$ represents complex elements of a vector or matrix, also $\mathrm{Y}\left[n_{1}\right]=\left[\mathrm{Y}_{1}\left[n_{1}\right], \mathrm{Y}_{2}\left[n_{1}\right], \ldots, \mathrm{Y}_{M}\left[n_{1}\right]\right]^{\mathrm{T}}$, $\mathbf{S}\left[n_{1}\right]=\left[S^{1}\left[n_{1}\right], S^{2}\left[n_{1}\right], \ldots, S^{K}\left[n_{1}\right]\right]^{\mathrm{T}}, \quad \mathbf{N}\left[n_{1}\right]=\left[N_{1}\left[n_{1}\right], N_{2}\left[n_{1}\right], \ldots, N_{M}\left[n_{1}\right]\right]^{\mathrm{T}} \quad$ and $\mathbf{H}\left[n_{1}\right]=\left[\begin{array}{ccc}H_{1}^{1}\left[n_{1}\right] \cdots H_{1}^{K}\left[n_{1}\right] \\ \vdots & \cdots & \vdots \\ H_{M}^{1}\left[n_{1}\right] \cdots & H_{M}^{K}\left[n_{1}\right]\end{array}\right]$ are the $X$-point FFTs of the corresponding time domain sequences, and ${ }^{\mathrm{T}}$ in the superscript denotes transpose of a vector.

\subsubsection{Rayleigh Faded Doubly Selective Channel Model}

In a built-up environment with No Line of Sight (NLOS) between the transmitter and receiver, the propagating radio wave could be broken into multiple components, either (a) due to scattering or diffraction if it encounters objects that are smaller in dimension compared to its wavelength, or (b) due to reflection if it encounters objects that are larger in dimension compared to its wavelength. In a highly cluttered environment, the envelope of the radio signal usually follows a Rayleigh distribution. Thus, in this model, the signal is assumed to undergo a Rayleigh fading channel. Figure 2.3 presents a model of $K$ signal sources passing through a multi-path Rayleigh fading channel.

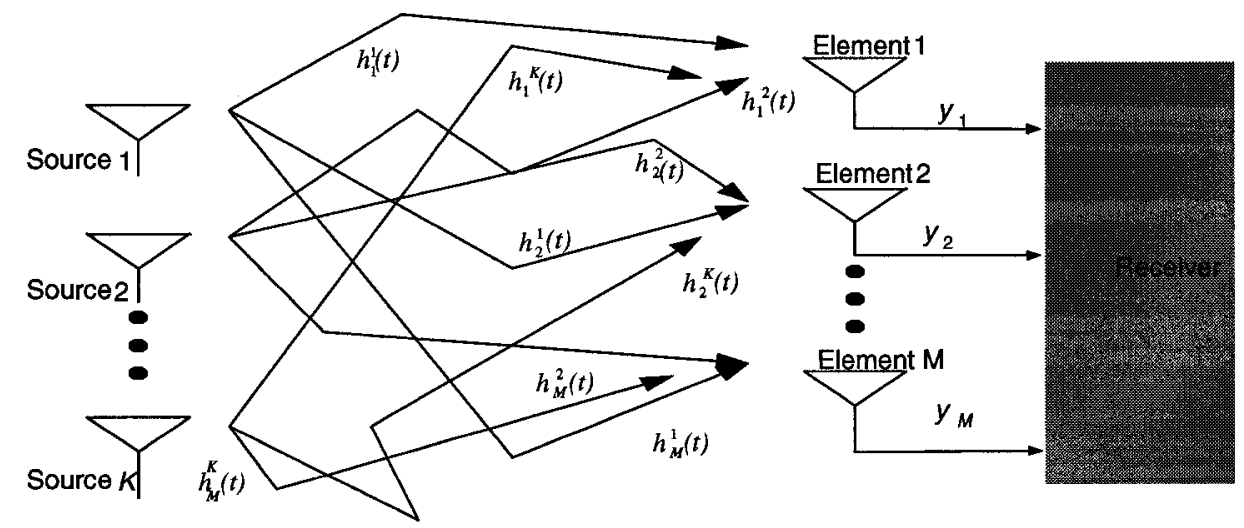

Fig. 2.3. A multi-user multi-path channel model.

In this model, we assume that the modulated signals of all sources are mutually uncorrelated with zero mean and unit variance and also uncorrelated with the noise. The 
output at the $m$ th antenna element can be expressed as given in (2.2). $b_{m}^{k}(t)$ is a zeromean complex Gaussian random impulse response associated with user $k$ and antenna element $m$. Fading components are independent and uncorrelated. When the long-term effects are considered, $\mathbf{h}_{m}(t)$ is modeled as:

$$
\mathbf{h}_{m}(t)=\left(\left(\frac{1}{\varsigma_{1}}\right)^{\Omega} b_{m}^{1}(t),\left(\frac{1}{\varsigma_{2}}\right)^{\Omega} b_{m}^{2}(t), \ldots,\left(\frac{1}{\varsigma_{K}}\right)^{\Omega} b_{m}^{K}(t)\right)^{\mathrm{T}}
$$

where $\varsigma_{k}$ is the distance of the user $k$ from the receiver for $k=1,2, \ldots, K, m=1,2, \ldots, M$, and $\Omega$ represents the propagation path loss exponent. Modified Smith's method [YB00] is used to generate the Rayleigh fading samples in simulations. A block diagram and procedure to implement the fading model is described in [YB00].

With the consideration of time varying multi-path channel, we consider a frequency selective Rayleigh fading channel, modeled by a tapped delay line with $L$ non-zero taps [Pro95] with a CIR

$$
h_{m}^{k}(t, \tau)=\sum_{l=0}^{L-1} h_{m}^{k}(t, l) \delta\left(\tau-\tau_{l}(t)\right)
$$

where $b_{m}^{k}(t, l)$ represents time-varying complex gain of the $l$ th path between $k$ th user and $m$ th antenna element and $\tau_{l}(t)$ is the $l$ th delay of the path. Random fluctuation of $b_{m}^{k}(t, l)$ is caused by the scattering or diffraction and can be modeled as a zero mean Wide Sense Stationary (WSS) Gaussian random variable. The CFR is obtained by taking the FFT of the CIR.

The channel model mostly used in this thesis is a 20-path C2-Urban Macro channel [Win01] defined by the WINNER project for a wide area scenario (other than the SUI-5 three path channel model which is used in Appendix B). The average PDP of the C2Urban Macro channel is given in Table 2.1. 
Table 2.1 Average PDP of C2-channel [Win01].

\begin{tabular}{|c|c|}
\hline Average Power [dB] & Delays in microseconds $[\mu \mathrm{sec}]$ \\
\hline-0.50 & 0 \\
0 & 0.005 \\
-3.40 & .135 \\
-2.80 & .160 \\
-4.60 & .215 \\
-0.90 & .260 \\
-6.70 & .385 \\
-4.50 & .400 \\
-9.00 & .530 \\
-7.80 & .540 \\
-7.40 & .650 \\
-8.40 & .670 \\
-11.00 & .720 \\
-9.00 & .750 \\
-5.10 & .800 \\
-6.70 & .945 \\
-12.10 & 1.035 \\
-13.20 & 1.185 \\
-13.70 & 1.390 \\
-19.80 & 1.470 \\
\hline
\end{tabular}

A snapshot in Figure 2.4 shows the doubly selective nature of the simulated channel. A total of 416 sub-carriers are used $(B=416)$ and 120 consecutive blocks $(F=120$, where $F$ is the number of blocks in a frame) are shown as an example with a vehicular speed of 50 $\mathrm{km} / \mathrm{hr}$.

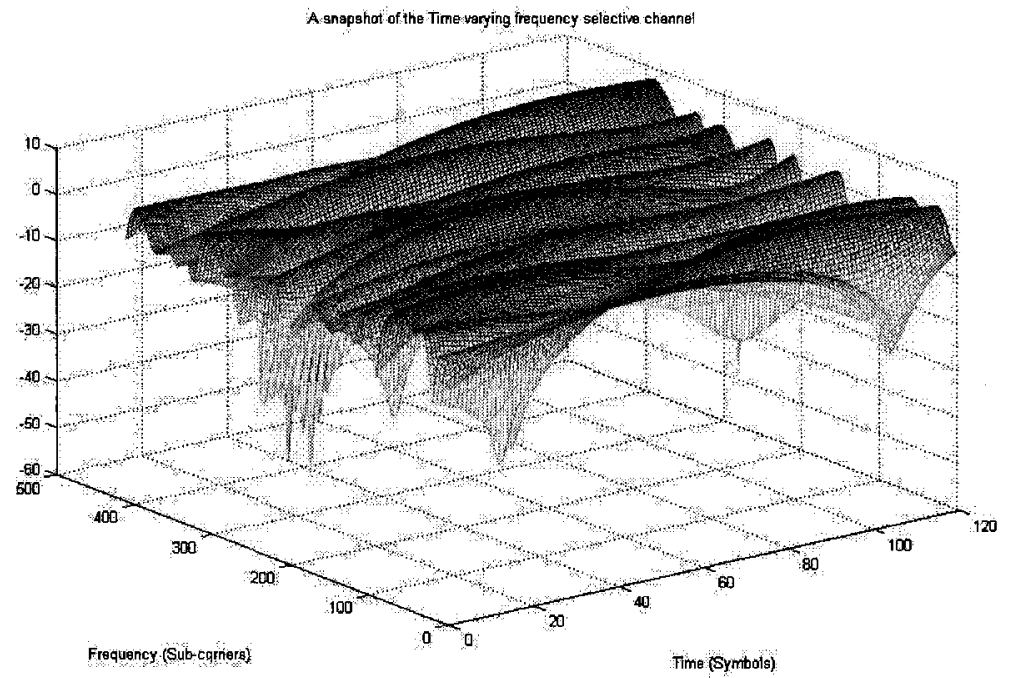

Fig. 2.4. Snapshot of time-frequency variation of the channel (one frame with $F=120$ ). 
The fading samples created above could be used for simulating multiple consecutive frame transmission; for disjoint transmission (having sleep mode in between the blocks), different fading samples should be used in each frame as shown in Figure 2.5 with an assumption that a frame consists of 12 blocks $(F=12)$.

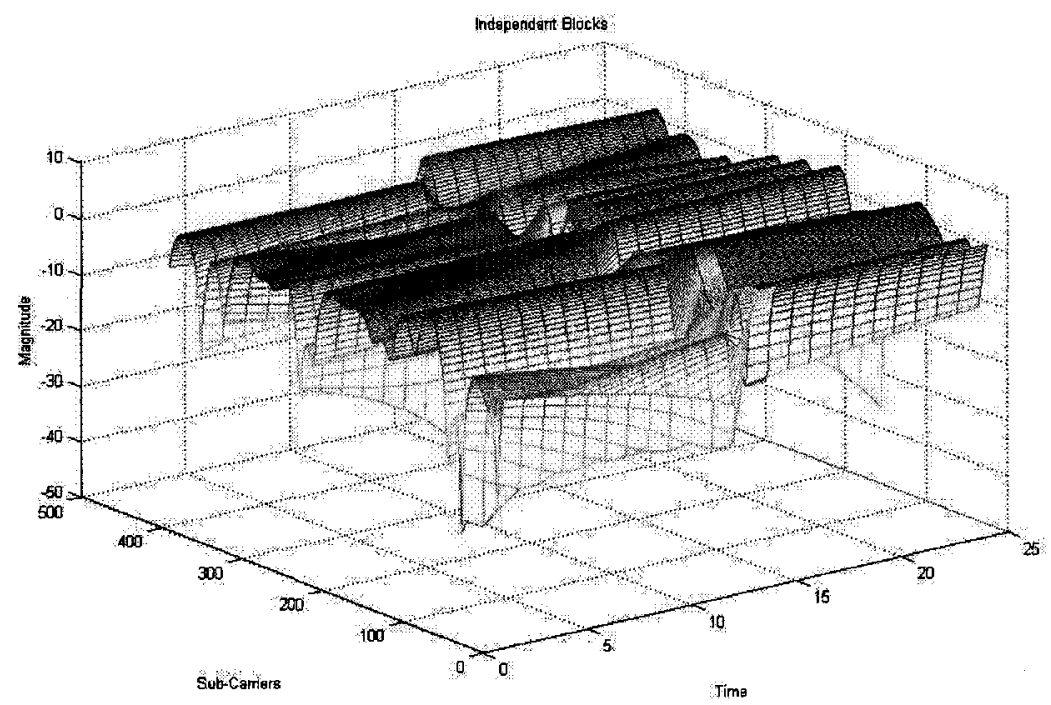

Fig. 2.5. Snapshot of time-frequency variation for disjoint transmission (two frames each has $F=12$ ).

\subsection{Frequency Domain Linear Equalization and Adaptation}

In this section, we briefly describe the Linear Equalization (LE) and two basic approaches of the equalizer adaptation techniques for SC-FDE systems. The SC-FDE modulation is also referred to as Serial Modulation (SM) or DFT-precoded OFDM in the Generalized Multi-Carrier (GMC) context [Win02]. This leads to the similarity of SCFDE system with OFDM. Therefore, in a SC-FDE system, the time-varying frequency selective channel can be transformed into a set of parallel time-varying frequency flat channels (so-called sub-carriers). Hence on each sub-carrier, we may apply existing frequency domain equalization techniques. Frequency domain channel equalization for SC-FDE is considered in [FAB02, SKJ9524, TB04 and references therein].

In a multi-element receiver based on FDE, there could be two approaches to compute the antenna array coefficients to tackle the equalization and/or interference suppression problem. In the first, the coefficients are directly computed from the received antenna 
array output error using a training sequence or receiver decisions as reference [Hay02]. In the second, we compute the array weighing coefficients from the estimates of the multilink CFRs. We call the former direct adaptation and the latter indirect adaptation [Hay02]. One might think that direct adaptation and indirect adaptation would perform in the same way in equalizer weight update. However, as they use the training data in different ways, they may perform differently [Zie91].

\subsubsection{Direct Adaptation using Decisions or Pilots}

In the direct adaptation technique the $\mathrm{CE}$ is implicit, producing the estimates of the equalizer coefficients directly, for example Least Squares (LS) or Recursive Least Squares (RLS) algorithms. We define the estimated equalizer coefficients as $\hat{\mathbf{W}}[n] \in$ $C^{M \times K}$, by using the hard decisions $\tilde{\mathbf{S}} \in C^{K x B}$ or pilot symbols (denoted as $\overrightarrow{\mathbf{A}} \in C^{K x T r}$ ), we calculate the coefficients with the Least Squares (LS) optimization criteria as

$$
\hat{\mathbf{W}}[n]=\left\langle\mathbf{Y}[n] \mathbf{Y}[n]^{\mathrm{H}}\right\rangle^{-1}\left\langle\mathbf{Y}[n] \tilde{\mathbf{S}}[n]^{\mathrm{H}}\right\rangle
$$

where $<>$ means time average over several DFT-precoded OFDM blocks $(F),{ }^{\mathrm{H}}$ in the superscript denotes Hermitian transpose and $n$ is the frequency index for $n=0,1, \ldots, B-1$. A similar approach is used in one of the proposed interference suppression techniques described in Chapter 6.

Following are a few points about the direct adaptation:

- Directly adapting the array coefficient is well suited to decision-directed adaptation.

- With the direct adaptation method $\hat{\mathbf{W}}[n]$ can be updated by means of any of the existing adaptation algorithms such as Least Mean Squares (LMS), Constant Modulus Algorithm (CMA) or exponentially weighted Recursive Least Squares (RLS) algorithm at each frequency.

- In direct adaptation, a condition of implementation is that all the users or interferers should be present in each received signal.

- One of the key issues associated with the direct adaptation is its convergence characteristics. 
- Speed of convergence depends on the suitable conditions to cause less error propagation.

- The number of blocks available for time averaging also affects the direct adaptation performance.

- There is no need to estimate properties or numbers of interferers.

\subsubsection{Indirect Adaptation with Perfect (Known) Channel State Information}

In indirect frequency domain equalizer adaptation we utilize the channel frequency responses either known to the receiver (this section) or their estimated values (Section 2.6.3). The Linear MMSE multi-user spatial processing uses the antenna weights such that the power of the differential signal between the combiner output and a reference signal, which is characteristic of the desired user, is minimized, that is: $E\left(\left|\hat{s}^{k}(q)-s^{k}(q)\right|^{2}\right)$, where $s^{k}(q)$ represents the $k$ th user data symbol and $\hat{s}^{k}(q)$ is the corresponding estimate. The solution is given by the well-known Wiener Hopf equation, which can be directly solved in order to yield the optimum weights. The antenna array weighing coefficients of the frequency domain Linear Equalization (LE), $\mathbf{W}[n] \in \mathrm{C}^{M \times K}$ in case of Perfect Channel State Information (PCSI) are given by [Hay02]:

$$
\mathbb{W}[n]=\left[\mathbf{H}[n] \mathbf{H}[n]^{\mathrm{H}}+\sigma_{n}^{2} \mathbf{I}_{M}\right]^{-1} \mathbf{H}[n], \quad n=0,1, \ldots, B-1
$$

where $\sigma_{n}^{2}$ is the noise variance, $\mathbf{I}_{M}$ is an identity matrix of size $M, \mathbf{H}[n] \in C^{M \times K}$ and ${ }^{\mathrm{H}}$ denotes Hermitian transpose. As from (2.3), the frequency domain antenna array input vector $\mathbf{Y}[n] \in C^{M \times 1}$, the frequency domain equalizer output is given by:

$$
\mathbf{V}[n]=\mathbf{W}[n]^{\mathrm{H}} \mathbf{Y}[n]
$$

where $n=0,1, \ldots, B-1$ is the frequency index. The equalizer time domain output samples are given by:

$$
\mathbf{v}(q)=\mathbf{F}_{B}^{-1}(\mathrm{~V}[n])
$$

where $\mathbf{F}_{B}^{-1}$ represents $B$-point IFFT. The corresponding MMSE for LE is given by: 


$$
J_{\min }=\frac{1}{B^{*} K} \sum_{n=0}^{B-1} \operatorname{tr}\left[\left(\mathbf{W}[n]^{\mathrm{H}} \mathbf{H}[n]-\mathbf{I}_{K}\right)\left(\mathbf{W}[n]^{\mathrm{H}} \mathbf{H}[n]-\mathbf{I}_{K}\right)^{\mathrm{H}}+\sigma_{n}^{2} \mathbf{W}[n] \mathbf{W}[n]^{\mathrm{H}}\right](2
$$

where $t r$ represents the trace of a matrix.

\subsubsection{Indirect Adaptation using Estimated CFR}

To compute the equalizer coefficients with the estimated CFRs, the known CFRs in the MMSE equalizer's coefficient equation (2.7) are replaced with the estimates, as given below:

$$
\hat{\mathbf{W}}[n]=\left[\hat{\mathrm{H}}[n] \hat{\mathbf{H}}[n]^{\mathrm{H}}+\sigma_{n}^{2} \mathbf{I}_{M}\right]^{-1} \hat{\mathbf{H}}[n]
$$

where $\hat{\mathbf{H}}[n] \in C^{M \times K}$ is the estimated channel matrix at frequency $n$ for all $K$ interfering signals. In indirect adaptation, the $\mathrm{CE}$ is done explicitly to produce the estimates of the CFRs between the sources and the receiving antennas. Following are a few points about the indirect adaptation:

- $\hat{\mathbf{H}}[n]$ is a result of interpolation in frequency and time from pilot measurements on the pilot grid. We need interpolation in the frequency axis if the pilots are scattered among the data symbols. Moreover, time interpolation is necessary due to time variation of the channel.

- Indirect adaptation requires explicit channel estimates of all interfering user signals.

- Estimation process does not require that all pilots be transmitted simultaneously, and is therefore well suited to separate (orthogonal) transmission of pilots.

- Separate estimation of noise variance $\sigma_{n}^{2}$ is necessary.

\subsection{Pilot Multiplexing}

We employed a pilot or training sequence-based CE in multi-user SC-FDE system. CE using pilot symbols is employed in current $3^{\text {rd }}$ Generation (3G) systems [Kor03] and will most likely be applied in the proposed $4 \mathrm{G}$ or beyond $3 \mathrm{G}$ wireless communication systems [Win03]. In order to estimate interference-prone channels, we need to design training sequences with good correlation properties. We will show that the proper choice of $\mathrm{Chu}$ 
sequences [Chu72] may ease the problem of estimation in multi-user scenarios. In order to estimate the channel in a multi-user SC-FDE system, different training sequences or pilot placements are compared. Training could be multiplexed with data, either (a) in time-multiplexed fashion, in which short training blocks are multiplexed with data blocks in time, which we call Time Multiplexed Training (TMT), or (b) in frequency multiplexed fashion, in which pilots are inserted into the data block, which we call Frequency Multiplexed Training (FMT). For FMT, we have to use the GMC transceiver structure described in Section 2.5.1, to insert the pilot tones among the data symbols. Moreover, it will be shown that $\mathrm{CE}$ with inter-user interference could be improved by keeping the training sequences of ICUs orthogonal to each other. We have compared a few such choices with which we can improve the multi-user channel estimates.

\subsubsection{Time Multiplexed Training (TMT)}

In Time Multiplexed Training (TMT), consecutive short training blocks preceded by CPs are sent. Since the CIR is just a fraction of the block duration, we could employ training blocks that are shorter than the standard data block. The length of these blocks is at least equal to or greater than the expected maximum channel delay spread $(L)$. The concept is depicted in Figure 2.6. CP for training and data blocks are omitted for the sake of simplicity. A short block ' $\mathrm{T}_{\mathrm{r}}$ ' in the beginning denotes the block containing the training sequence. As we are dealing with blocks that appear periodic, (since they have CPs), we can imagine repeating the length- $T_{r}$ training sequence $B / T_{r}$ times in order to make a total block of length $B . T_{r}$ denotes the length of the short block. The FFT of this block is a $B$ point sequence, which is zero except at the $T_{r}$ "sample points“, spaced at intervals of $B / T_{r}$. These are the $T_{r}$ "training pilots", distributed uniformly in the block of $B$ frequencies.

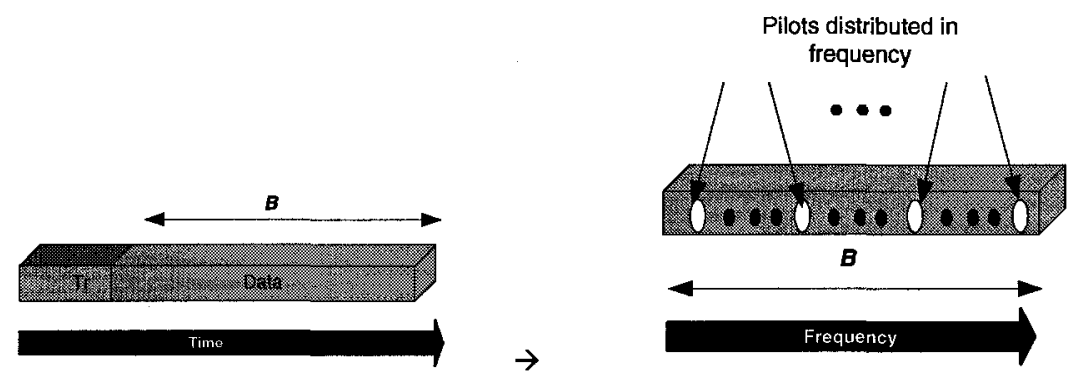

Fig. 2.6. Concept of Time Multiplexed Training (TMT). 
Notice that the channel estimates can be improved by time averaging over several such short training blocks. To achieve better $\mathrm{CE}$, different Chu sequences should be used in consecutive blocks.

\subsubsection{Frequency Multiplexed Training (FMT)}

The second pilot multiplexing method is in frequency domain. In SC-FDE with the GMC structure, we can insert pilots in the frequency domain at any sub-carrier to form a desirable pilot grid, exactly the same way as in OFDM. This is the reason why SC-FDE is also known as DFT-precoded OFDM. The pilot insertion is depicted in Figure 2.7. In Figure 2.7 pilots are denoted by ' $T$ '.

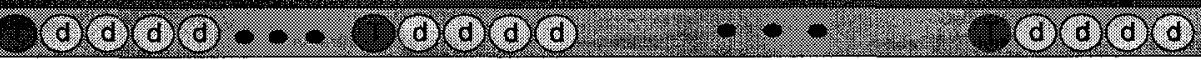

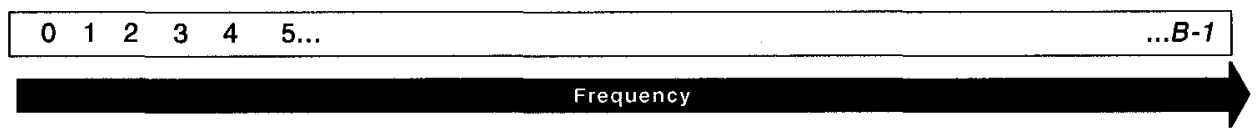

Fig. 2.7. Concept of Frequency Multiplexed Training (FMT).

There are two approaches to pilot insertion in frequency domain: Frequency-Expanding Technique (FET) and Frequency-Domain Superimposed Pilot Technique (FDSP) [LDF06] or also known as implicit or superimposed pilots [LLM04]. In the former, the data carriers are shifted to accommodate the pilot tones. In FDSP, pilot tones are superimposed with some scaling, on the data sub-carriers. Both schemes have their own merits and demerits. FDSP is spectrum efficient but has some performance degradation [LDF06]. One of the problems associated with the FDSP is the interference between the data and the pilots. Also the channel estimates are corrupted by the data resulting in an irreducible noise floor. Because of the performance superiority, we will be considering FET called here FMT technique with an extension to multi-user scenario. 


\subsubsection{Comparison of TMT and FMT}

In this section we will compare the two pilot multiplexing choices described above. The main advantage of using FMT is that estimation process is done in a single block, compared to the TMT in which short training blocks are sent along with the data blocks. The advantage of TMT is its superior PAPR. As in the TMT, short training blocks have CPs; therefore, TMT has a slightly higher overhead penalty. A disadvantage of FMT is that the resulting data plus frequency-multiplexed pilot waveform, is no longer a pure single carrier waveform, and therefore has a slightly higher PAPR than a data waveform without pilots or a TMT waveform. As a result, the data power in transmitted blocks containing FMT pilots must be backed off about an extra 1 to $1.5 \mathrm{~dB}$ to satisfy typical power spectrum mask requirements [LDF06].

\subsubsection{Optimal Training Sequence}

Selection of proper training sequences to be used as pilots is crucial in CE process. The training sequences to be used in the TMT or FMT training schemes should be chosen with the objective of minimizing the MSE of the estimated CFR. Such a desired training sequence having constant envelope in time domain is good to encounter the PAPR, and also their flat spectrum, which is favorable in frequency domain CE process. These properties are found in a well-known $\mathrm{Chu}$ sequence [Chu72]. Chu sequences are generated according to the procedure given in [Chu72]. The following equations are used to generate Chu sequences for $T_{r}$ length of training sequence:

$$
\begin{array}{ll}
a^{k}(\bar{q})=\exp \left(j \vec{q} \pi \bar{q}^{2} / T_{r}\right) & \text { for even } T_{r} \\
a^{k}(\bar{q})=\exp \left(j \vec{q} \pi \bar{q}(\bar{q}-1) / T_{r}\right) & \text { for odd } T_{r}
\end{array}
$$

where $\bar{q}=0,1, \ldots, T_{r}-1$ is the discrete time index, $k=1,2, \ldots, K$ is the source or user index and $\vec{z}$ represents prime numbers relative to $T_{r}$. In this thesis we always use an even $T_{r}$. For the multi-user case a base Chu sequence is generated for one transmitter-receiver link, and sequences for the other links are phase-rotated versions of the base sequence [SCP04]. Phase-rotation in time gives a shift in the frequency domain pilots, making them orthogonal in frequency domain. We will be using this approach for generating 
training sequences in order to deal with known orthogonal interferers and is described in Chapter 3.

\subsubsection{Initial Channel Estimates}

To obtain the frequency domain initial pilot-based short estimate of the CFR, timemultiplexed short training blocks in the case of TMT, or scattered pilots in the case of FMT are used. We call such channel estimates "short" since the pilot symbols are sparsely placed in a pilot-carrying block and $T_{\mathrm{r}}<B$, where $T_{\mathrm{r}}$ is the total number of pilots in one block and $B$ is the block length. A simple method to estimate the CFR is based on Least Squares (LS) principle [JHS95], [SNT03], [KSN03] and [CM98]. Short training blocks are sent from the transmitter in TMT or the pilots scattered in FMT, and by performing simple element by element division on the received vector we obtain the initial short estimate as follows:

$$
\breve{\mathbf{H}}[\bar{n}, \bar{c}]=\frac{\breve{\mathbf{Y}}[\bar{n}, \bar{c}]}{\overline{\mathbf{A}}[\bar{n}]}
$$

where $\bar{n}=0,1, \ldots, T_{r}-1$, is the pilot frequency index, $\bar{c}$ represents the index for the pilotcarrying blocks in a frame, for example in Figure 2.8, $\bar{c}$ could be 1 and 12 representing the first and twelfth pilot-carrying block in a 12-block frame, $\breve{\mathbf{Y}}[\bar{n}, \bar{c}] \in C^{M \mathrm{x} 1}$ is the array input during the training phase, $\breve{\mathbf{H}}[\bar{n}, \bar{c}] \in \mathrm{C}^{M \times K}$ is the short channel estimate at $\bar{n}$ th frequency and $\overrightarrow{\mathrm{A}}[\bar{n}] \in C^{K \mathbf{x} T r}$ is the FFT of the Chu training sequences of all the $K$ users such that

$$
\overrightarrow{\mathbf{A}}=\left[\overrightarrow{\mathbf{A}}^{1}, \overrightarrow{\mathbf{A}}^{2}, \ldots, \overrightarrow{\mathbf{A}}^{K}\right]^{\mathrm{T}}
$$

and

$$
\overrightarrow{\mathbf{A}}^{k}=\left[\vec{A}_{0}^{k}, \vec{A}_{1}^{k}, \ldots, \vec{A}_{T,-1}^{k}\right]
$$

for $k=1,2, \ldots, K$. After getting short CFRs, interpolation is required in both frequency and time axes to obtain estimates for the whole time-frequency grid. 


\subsubsection{Interpolation}

In order to get a full length estimate of the CFRs $\hat{\mathbf{H}}[n, \bar{c}] \in \mathrm{C}^{M \times K}$ for $n=0,1, \ldots, B-1$, frequency and time axes interpolation is carried out. There are several interpolation techniques available in the literature, which have their own benefits and drawbacks in terms of performance and complexities [HKR97] and [SJS03]. An existing interpolation method for CE for any multi-carrier system can be employed in a SC-FDE system.

\subsubsection{Pilot Grid Selection}

We define a frame, consisting of $F$ blocks, each having $B$ frequencies. This forms a timefrequency grid as shown in Figure 2.8.

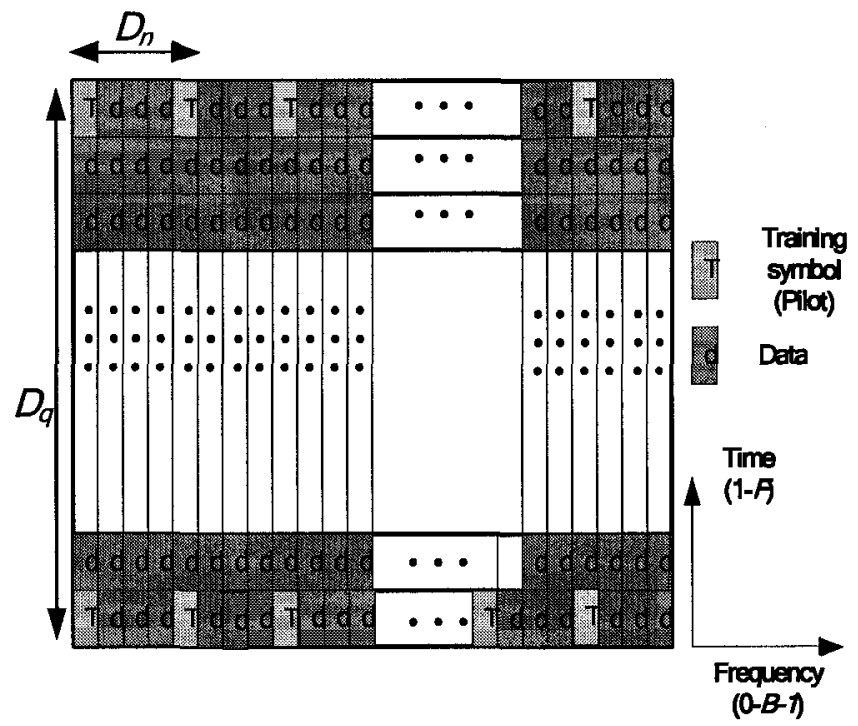

Fig. 2.8. Single user pilot grid (a frame).

The spacing between the pilots depends on the variation of the channel in time and frequency. Nyquist sampling theorem dictates the inter-pilot spacing. In order to find the minimum pilot spacing in time and frequency, expected bandwidth of the channel variation in time and frequency should be known. Doppler spread and the maximum delay spread of the channel give us this information. In general the requirements for the pilot spacing are given by [Par04], [NES97]: 


$$
D_{n}<\frac{1}{L} \text { and } D_{q}<\frac{1}{2 f_{D \max }}
$$

where $L$ represents the maximum delay spread of the channel, $f_{D \max }$ is the maximum expected Doppler frequency and $D_{n}$ and $D_{q}$ represent pilot spacing in frequency and time. Moreover, the number of pilots also depends on the compromise between the required data rate (pilot overhead) and the estimation performance.

\subsubsection{FFT/IFFT Interpolation and Smoothing}

A straightforward and simple interpolation technique is based on IFFT/FFT. A Low Pass (LP) smoothing filter is also used to further improve the channel estimates [YA97]. This technique uses a LP filtering in a so-called transform domain. Following are the steps to obtain an interpolated CFR from pilot-based, sparsely sampled short channel estimates:

- Convert the short channel estimates $\breve{\mathbf{H}}[\bar{n}, \bar{c}] \in \mathrm{C}^{M \times K}$ into time domain by IFFT, where $\bar{n}=0,1, \ldots, T_{r}-1$ and $\bar{c}$ represents the index for the pilot-carrying blocks in a frame.

- Pad the resulting time domain samples to the length of the block $(B)$ with zeros.

- Take the FFT of the resulting zero-padded sequence to obtain the full-length CFR. The sparsely sampled short estimated CFRs are converted into time domain by using IFFT as:

$$
\ddot{\mathbf{g}}=\mathbf{F}_{T r}^{-1}(\breve{\mathbf{H}})
$$

where $\mathbf{F}_{T r}^{-1}$ represents a $T_{r}$-point IFFT and $\ddot{\mathbf{g}}(\bar{q}) \in C^{M \times K}$ for $\bar{q}=0,1, \ldots, T_{r}-1$. The initial portion of the resulting estimated Impulse Response (IR) can be viewed as the signal region while the noise component is spread over the remaining region. The LP filtering is done in this domain by putting zeros all over, other than in the so-called signal region:

$$
\ddot{\mathbf{g}}_{m}(q, \bar{c})=\left\{\begin{array}{lc}
\ddot{\mathbf{g}}_{m}(\bar{q}, \bar{c}) & 0<q<I_{r} \\
0 & \text { Otherwise }
\end{array}\right.
$$

where $\bar{q}$ is the sample index in the transformed domain, $I_{r}$ is the span of the signal space defined in number of samples, $m$ is the index for the antenna elements for $m=1,2, \ldots, M$ 
and $q=0,1, \ldots, B-1$. The $I_{r}$ can also be visualized as the cutoff of the LP filter. Full length interpolated estimated CFR for each of the pilot-carrying block in a frame is obtained as:

$$
\hat{\mathbf{H}}=\mathbf{F}_{B}(\ddot{\bar{g}})
$$

where $\mathbf{F}_{B}$ represents a $B$-point FFT. Windowing out the first $I_{r}$ fingers in the CIR and transferring them back to the frequency domain will reduce the noise variance in the $\mathrm{CE}$ [YA97].

Figure 2.9 shows the re-growth in obtaining the initial CE caused by the interpolation errors. In these simulations, there are 104 pilots $\left(T_{r}=104\right)$ in a block of 416 sub-carriers $(B=416)$.

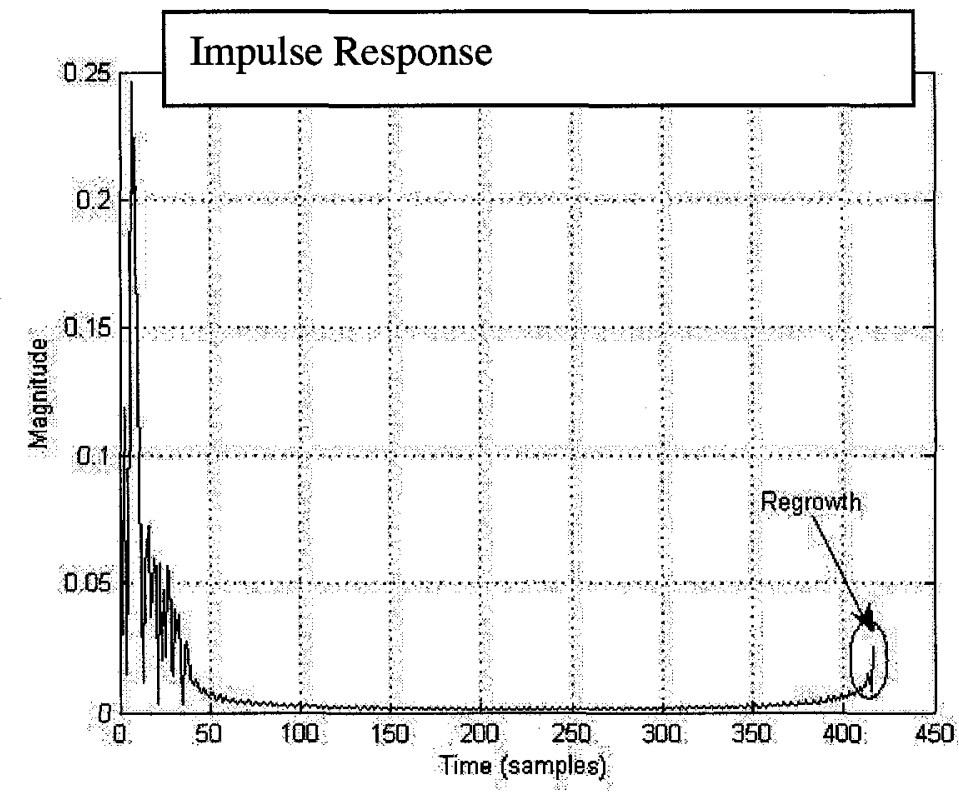

Fig. 2.9. Re-growth in the estimated CIR.

Figure 2.10 shows the process of LP windowing filter and how the re-growth is suppressed by filling in the zeroes. Figures 2.11 and 2.12 show the smoothing effects of the LP filter on the estimated CFR. 


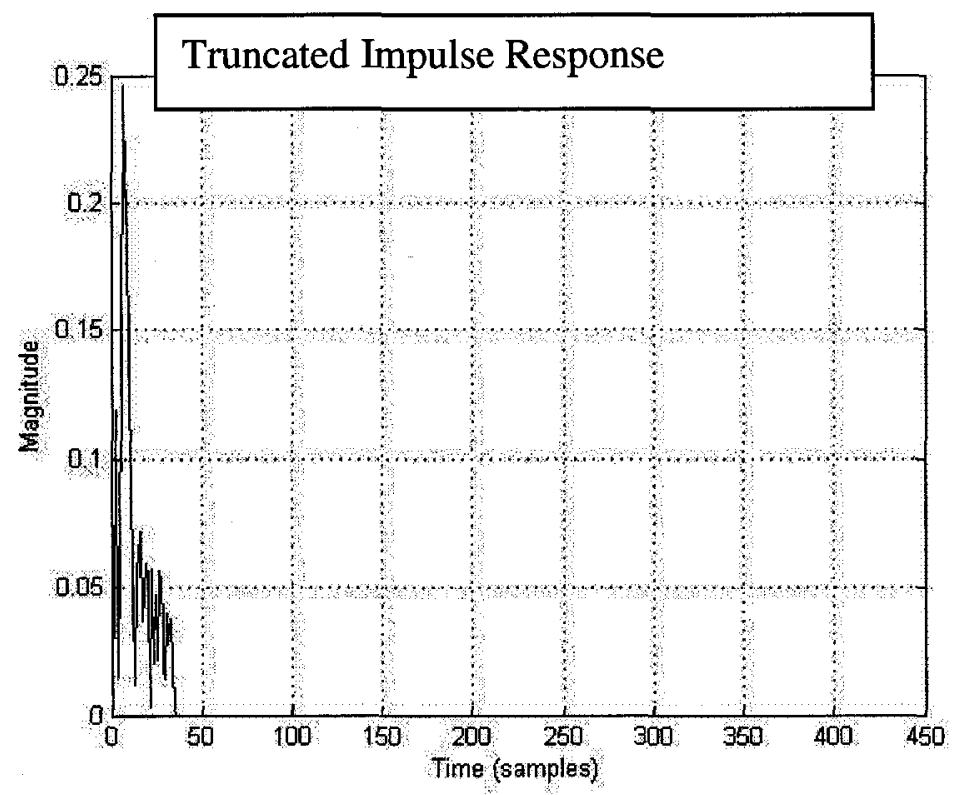

Fig. 2.10. Effect of filtering on the estimated CIR.

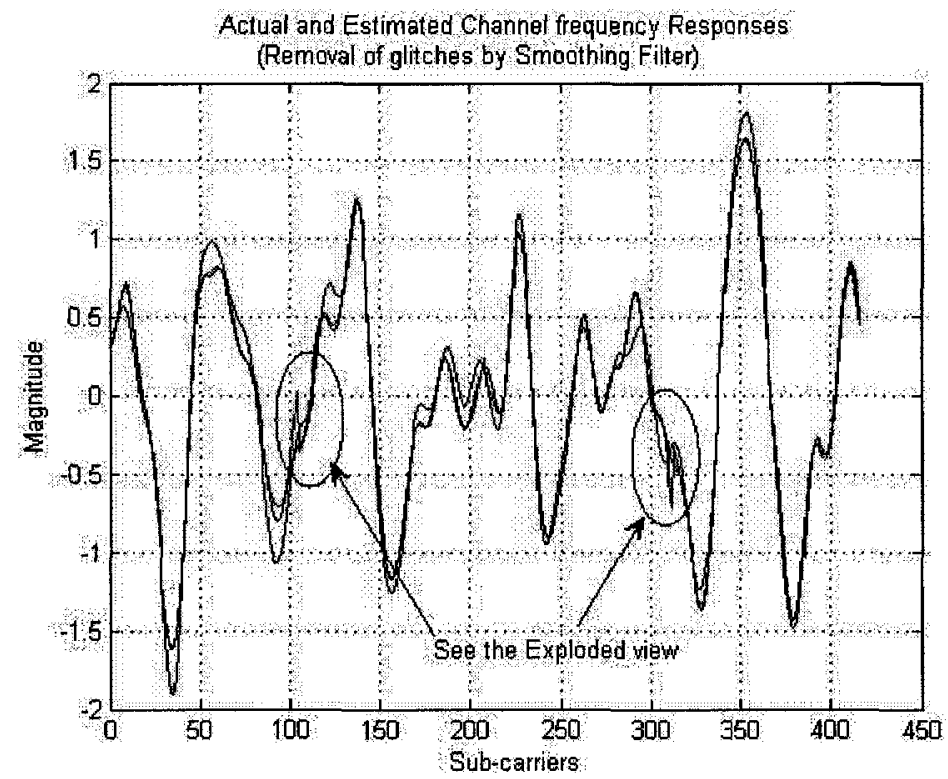

Fig. 2.11. Actual and estimated CFR with and without LP smoothing filter. 

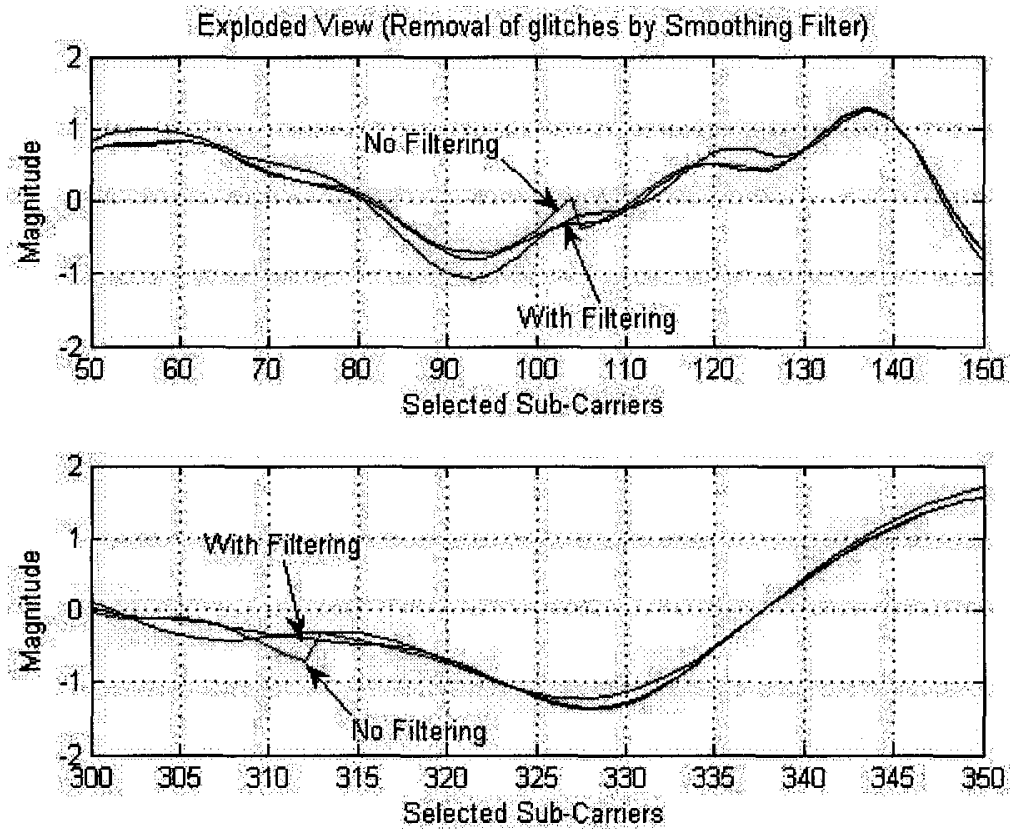

Fig. 2.12. Exploded view of the actual and estimated CFR with and without LP smoothing filter.

\subsubsection{3. $2 x 1 D$ Wiener Interpolation}

Two-dimensional (2-D) interpolation based on Wiener filtering is a well-known interpolation method [HKR97]. In this method, a 2-D interpolation matrix is computed for whole of the time-frequency grid. From an implementation point of view the computation of the full 2-D interpolation matrix is too complex. A well-known solution is to break the 2-D interpolation problem into two cascaded 1-D filters, one working in the frequency direction and the other in time. It is called $2 \times 1 D$ or separable Wiener interpolation filter [HKR97]. Let the FIR filter in frequency and time direction be denoted by $\boldsymbol{\Phi}^{\prime \prime}[n]=\left[\Phi_{1}^{\prime \prime}[n], \Phi_{2}^{\prime \prime}[n], \ldots, \Phi_{Q_{n}}^{\prime \prime}[n]\right]^{\mathrm{T}} \quad$ and $\quad \boldsymbol{\Phi}^{\prime}[q]=\left[\Phi_{1}^{\prime}[q], \Phi_{2}^{\prime}[q], \ldots, \Phi_{Q_{q}}^{\prime}[q]\right]^{\mathrm{T}}$ respectively, then the estimator for $2 \times 1 \mathrm{D}$ solution is obtained by using the Kronecker product, so $\boldsymbol{\Phi}[n, q]=\boldsymbol{\Phi}^{\prime \prime}[n] \boldsymbol{\Phi}^{\prime}[q]$ of dimension $Q_{n} \times Q_{q}$, where $Q_{n}$ and $Q_{q}$ are the number of pilots available in the sliding window defined later in this section. The optimal filter in the sense of minimizing the MSE is the Wiener filter [Kay93]. The estimators $\boldsymbol{\Phi}^{\prime \prime}[n]$ and $\boldsymbol{\Phi}^{\prime}[q]$ are obtained by solving the Wiener-Hopf equation in the frequency and time directions, respectively [HKR97]. The filters $\boldsymbol{\Phi}^{\prime \prime}[n]$ and $\boldsymbol{\Phi}^{\prime}[q]$ are designed such that 
they cover a variety of power delay profiles and Doppler power spectra. A rectangular shaped PDP with maximum delay and a rectangular shaped Doppler power spectrum with maximum Doppler frequency are used. The parameters of the robust estimator should always be equal to or larger than the worst case channel conditions, i.e. largest propagation delays and maximum expected velocity of the mobile user. Furthermore, the average SNR at the array input, which is used to generate the filter coefficients should be equal or larger than actual average SNR.

In our $2 \times 1 \mathrm{D}$ interpolation filters, the filter coefficients of Wiener filter with so called model mismatch are generated with the following prior knowledge about channel statistics:

- The maximum delay of the channel is known.

- The maximum expected velocity is also known to the receiver.

- SNR is assumed to be perfectly known.

In order to estimate full length CFRs, we may use all the pilots' correlations available in the block. But this involves inversion of a larger matrix, resulting in a greater computational complexity. Therefore, a 2D window of shorter size is defined, covering fewer pilots $\left(Q_{n}\right.$ or $\left.Q_{q}\right)$. In 2x1D interpolation, we may start either with frequency axis filtering or the time axis, as the order of filtering does not matter due to the linearity of the process. The filtering in the frequency direction is obtained by multiplying the Wiener filter coefficients with the short estimates as [SJS03]:

$$
\tilde{\mathbf{H}}[n]=\sum_{c n=0}^{Q n-1} \boldsymbol{\Phi}^{\prime \prime}[n] \breve{\mathbf{H}}_{c n}
$$

where $\breve{\mathbf{H}}_{c n}$ represents the pilot-based estimates of the CFRs within the sliding window defined by $Q_{n}$ and $Q_{n}$ is the number of pilots available in the sliding window, $n$ is the frequency of interest, $c n$ is the nearest pilot position and $\boldsymbol{\Phi}^{\prime \prime}[n]=\left[\Phi_{1}^{\prime \prime}[n], \Phi_{2}^{\prime \prime}[n], \ldots, \Phi_{Q_{n}^{\prime \prime}}^{\prime}[n]\right]^{\mathrm{T}}$ is the filter coefficient. The filter coefficient vector is defined at each antenna element, computed for all the pilot-carrying blocks in a frame, given by:

$$
\boldsymbol{\Phi}^{\prime \prime}[n]=\left[\mathbf{R}_{\mathrm{H}_{\mathrm{p}} \mathrm{H}_{\mathrm{p}}}[n]+\sigma_{n}^{2} \mathbf{I}_{Q_{n}}\right]^{-1} \mathbf{R}_{\mathrm{HH}_{\mathrm{p}}}[n]
$$


where $\mathbf{R}_{\mathrm{H}_{\mathrm{p}} \mathrm{H}_{\mathrm{p}}}$ is the auto-correlation function $\in \mathrm{C}^{Q n \times Q^{n}}$ between pilots themselves and $\mathbf{R}_{\mathrm{HH}_{\mathrm{p}}}$ is the cross-correlation vector $\in \mathrm{C}^{Q n x I}$ between pilots and the actual channel response. By assuming that the data symbols have zero mean and are statistically independent from the pilots, the cross-correlation function is equal to the discrete timefrequency cross-correlation function

$$
\mathbf{R}_{\mathrm{HH}_{\mathrm{p}}}[n]=\left[\mathrm{R}_{n}\left[n-n_{1}\right], \cdots, \mathrm{R}_{n}\left[n-n_{Q_{n}}\right]\right\rfloor
$$

the auto-correlation function is given by:

$$
\mathbf{R}_{\mathrm{H}_{\mathrm{p}} \mathrm{H}_{\mathrm{p}}}=\left[\begin{array}{l}
\mathrm{R}_{n}[0] \cdots \mathrm{R}_{n}\left[n_{1}-n_{Q^{n}}\right] \\
\vdots \\
\mathrm{R}_{n}\left[n_{Q^{n}}-n_{1}\right] \cdots \mathrm{R}_{n}[0]
\end{array}\right]
$$

The cross-correlation function depends on the distances between the actual CE position $n$ and all pilot positions, while auto-correlation function is independent of the actual estimation position $n$ as it depends only on the distances between the pilot positions.

In order to compute the cross-correlation vector and auto-correlation matrix, prior knowledge of the maximum delay spread of the channel is assumed and is computed using the following [ESB96]:

$$
\mathrm{R}_{n}(\Delta n)=\frac{\left(1-e^{-L\left[\left(1 / \tau_{m s}\right)+2 \pi j(\Delta n / B)\right]}\right)}{\left(\tau_{m m s}\left(1-e^{-\left(n / \tau_{m s}\right)}\right)\right)\left[\frac{1}{\tau_{r m s}}+2 \pi \frac{\Delta n}{B}\right]}
$$

An approximation of uniform PDP and letting $\tau_{r m s} \rightarrow \infty$ further reduces the expression to

$$
\mathrm{R}_{n}(\Delta n)=\frac{\left(1-e^{-2 \pi L(\Delta n / \mathrm{B})}\right)}{2 \pi j \mathrm{~L}\left(\frac{\Delta n}{B}\right)}
$$

After filtering in the frequency axis, the full estimate i.e. $\tilde{\mathbf{H}}$ is available for every pilotcarrying block. Interpolation in time direction is carried out to obtain the complete estimates of the entire time-frequency grid i.e. $\hat{\mathbf{H}}$. For this purpose another Wiener filter $\boldsymbol{\Phi}^{\prime}[q]=\left[\Phi_{1}^{\prime}[q], \Phi_{2}^{\prime}[q], \ldots, \Phi_{Q_{q}}^{\prime}[q]\right]^{\mathrm{T}}$ is used in a similar way as described for the frequency axis. The difference is the different window size $\left(D_{q}\right)$. To compute the correlation functions, we assume uniform Doppler spectrum and prior knowledge of the maximum Doppler frequency. The auto and cross-correlation functions are computed by: 


$$
R_{q}(\Delta q)=J_{0}\left(2 \pi f_{D \max }(1+L / B) \Delta q\right)
$$

where $J_{0}$ is zeroth order Bessel's function of first kind, $f_{D \max }$ represents the maximum Doppler frequency and $B$ is the block size.

\subsection{Summary}

This chapter provides an introduction to the basic multi-user frequency domain equalization and $\mathrm{CE}$ techniques and a brief overview of some of the previous work. In essence, the chapter provides a framework in terms of equalization, adaptation, channel modeling and channel estimation techniques. This framework in turn gives us an overview of different basic approaches to be used in the rest of the thesis. (In this chapter, a multi-user signal and channel model is also presented. A wide or metropolitan area, urban macro channel, defined in the WINNER project is also described). 


\section{Chapter 3}

\section{Pilot Multiplexing in SC-FDE with Intra-Cell Interference}

\subsection{Introduction}

This chapter deals with the in-cell SDMA interference avoidance techniques. In this chapter we describe the uplink pilot placement and multiplexing techniques to deal with the intra-cell interference in cellular SC-FDE SDMA systems. We propose and evaluate orthogonal (non-overlapping) multi-user training signal generation and their placement by using either Time Multiplexed Training (TMT) or Frequency Multiplexed Training (FMT) and compare them with the non-orthogonal (overlapping) training. Least Squares (LS) Channel Estimation (CE) with Linear Equalization (LE) is used in the simulation results with different performance criteria in support of the proper selection and placement of the training sequences. Comparison of different interpolation techniques and effect of user mobility are also simulated. Finally, we present an analytical approach to analyze the simulation results with a Gaussian approximation in an environment with intra-cell interference.

\subsection{Inter-User Pilot Co-ordination in SC-FDE with Intra-Cell Interference}

In this section, we extend the concepts of pilot multiplexing either in time (TMT) or in frequency (FMT) described in Section 2.7 (Chapter 2) to multi-user in-cell SDMA scenarios. Throughout this thesis work, we assume that the users' data always overlap and hence interfere with each other, although they may or may not overlap during their training phases. Moreover, the training sequences of all the users may or may not be 
known to the receiver. Two possible multi-user pilot placement choices are compared: one with pilots that are overlapping from user to user, and one with orthogonal pilots. Therefore, the two possible pilot coordination schemes are:

1. Overlapping training

2. Orthogonal training

\subsubsection{Overlapping Training}

When pilots from In-Cell Users (ICUs) are transmitted simultaneously, they would overlap and interfere with one another. This could be termed as "No Coordination". For $\mathrm{CE}$, we assume that receiver knows the training sequences of every overlapping user at any specific time-frequency instance. This assumption is quite practical, as signal processing is carried out at the BS, and the BS is expected to have complete knowledge about the training of all the users in a cell.

With overlapping TMT schemes, there are mainly two possibilities. In the first, all users send their short training blocks at the same time with some space-time coding such as Alamouti precoding scheme (TMT-Al). Alamouti precoding produces orthogonal training with two users in the system. In the second possibility, we deal with more than two ICUs. In this case, we cannot create orthogonality with any space-time precoding. In this case, overlapping pilots must be constituted by special sequences having good correlation properties. In TMT with overlapping Chu sequences [Chu72] (TMT-Chu) or TMT with overlapping random QPSK symbols (TMT-QPSK), with $K$ ICUs in the system, we send and average over at least $K$ short training blocks. The overlapping (non-orthogonal) TMT concept with an assumption of three users, is depicted in Figure 3.1. In the figure ' $\mathrm{Tr}$ ' represents a short training block.

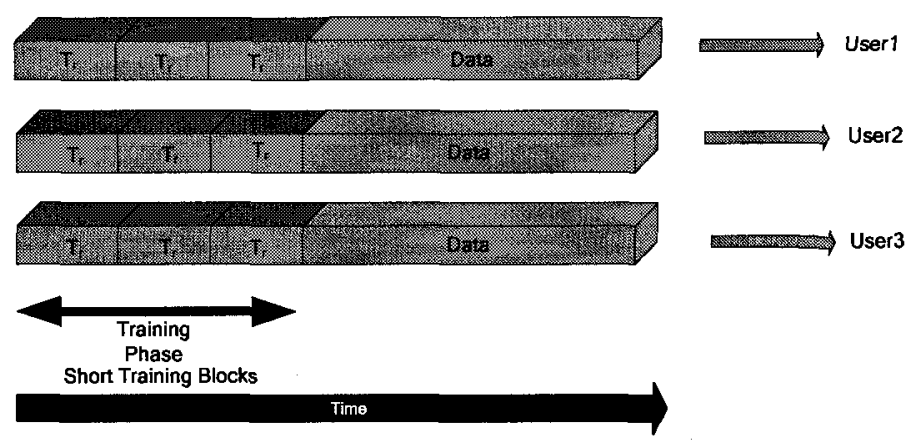

Fig. 3.1. Non-Orthogonal Time Multiplexed Training (TMT-Chu with three users). 
On reception of a short training block after FFT we have, at frequency $\bar{n}$,

$$
\breve{Y}_{m}[\bar{n}, \bar{c}]=\sum_{k=1}^{K} H_{m}^{k}[\bar{n}, \bar{c}] \vec{A}^{k}[\bar{n}]+N_{m}[\bar{n}, \bar{c}]
$$

where, $m=1,2, \ldots, M, \bar{c}$ represents the index for the pilot-carrying blocks in a frame and $\bar{n}=0,1, \ldots, T_{r}-1$, where $T_{r}$ is the training sequence length and $\breve{Y}_{m}[\bar{n}, \bar{c}]$ is the received signal during training at the $m$ th antenna element and $\vec{A}^{k}[\bar{n}]$ is the FFT of the Chu training sequence for the $k$ th user and $N_{m}[\bar{n}, \bar{c}]$ is the Gaussian noise.

\subsubsection{Least Squares (LS) Multi-User CE for Non-Orthogonal Training}

To obtain the initial pilot-based short estimates (assuming $T_{\mathrm{r}}<B$ ) of the channel transfer function for all in-cell overlapping users, joint estimation based on the Least Squares (LS) principle [JHS95], [SNT03], [KSN03] and [CM98] is used. For $K$ simultaneous pilots, LS estimation of the $K$ user channels requires at least $K$ pilot-based blocks per user in TMT and symbols per user in FMT. Here we give details with an example of TMT training. (A similar approach can be used for the FMT.)

Short training blocks are sent from each of the transmitters (ICUs), and by using the joint LS algorithm (described below), we form short time-averaged estimates of correlation matrices at each frequency $\bar{n}$. After getting the sparsely sampled frequency responses of all the users, 2x1D interpolation filters, as explained in Section 2.7.6.3 in Chapter 2 are used to obtain the estimates for the full time-frequency grid $(B x F)$, where $B$ is the total number of frequencies in a block and $F$ is the total number of blocks per frame.

\section{Initial CE:}

Consider the training sequence of $k$ th In-Cell User (ICU) $\vec{A}^{k}[\bar{n}]$ to be the FFT of the Chu sequence, while $\overrightarrow{\mathbf{A}}[\bar{n}]$ is the training vector of all users at frequency $\bar{n}$.

$$
\overrightarrow{\mathbf{A}}[\bar{n}]=\left[\vec{A}^{1}[\bar{n}], \vec{A}^{2}[\bar{n}], \ldots, \vec{A}^{K}[\bar{n}]\right]^{\mathrm{T}}
$$

With TMT we must have different training sequences in different blocks of the $k$ th user and the total number of training blocks $(N T)$ should at least be equal to the number of users $(K)$. The received signal at frequency $\bar{n}$, at antenna element $m$, during the training block $b$ for $b=1,2, \ldots, N T$ is given by: 


$$
\breve{Y}_{m}[\bar{n}, \bar{c}]_{b}=\sum_{k=1}^{K} H_{m}^{k}[\bar{n}, \bar{c}] \ddot{A}^{k}[\bar{n}]_{b}+N_{m}[\bar{n}, \bar{c}]_{b}
$$

in matrix form,

$$
\breve{\mathbf{Y}}[\bar{n}, \bar{c}]_{b}=\mathbf{H}[\bar{n}, \bar{c}] \mathbf{\mathrm { A }}[\bar{n}]_{b}+\mathbf{N}[\bar{n}, \bar{c}]_{b}
$$

$\bar{c}$ represents the index for the pilot-carrying blocks in a frame. The dimension of the vectors and matrices are given by:

$\breve{\mathrm{Y}}[\bar{n}, \bar{c}] \in \mathrm{C}^{M \times 1}, \mathrm{H}[\bar{n}, \bar{c}] \in \mathrm{C}^{M \times K}, \overrightarrow{\mathrm{A}}[\bar{n}] \in \mathrm{C}^{K \times 1}, \mathrm{~N}[\bar{n}, \bar{c}] \in \mathrm{C}^{M \times 1}$.

For a given channel matrix $\breve{\mathbf{H}}[\bar{n}, \bar{c}]$, the received signal $\breve{\mathbf{Y}}[\bar{n}, \bar{c}]$ is assumed to be Gaussian. The likelihood function will be:

$$
f(\breve{\mathbf{Y}}[\bar{n}, \bar{c}] \mid \breve{\mathbf{H}}[\bar{n}, \bar{c}])=\exp \left\{-\frac{1}{\sigma_{n}^{2}} \frac{1}{N T} \sum_{b=1}^{N T}\left(\left[\breve{\mathbf{Y}}_{b}[\bar{n}, \bar{c}]-\breve{\mathbf{H}}_{b}[\bar{n}, \bar{c}] \overrightarrow{\mathbf{A}}_{b}[\bar{n}]\right]^{\mathrm{H}}\left[\breve{\mathbf{Y}}_{b}[\bar{n}, \bar{c}]-\breve{\mathbf{H}}_{b}[\bar{n}, \bar{c}] \overrightarrow{\mathbf{A}}_{b}[\bar{n}]\right]\right)\right\}
$$

where $(.)^{\mathrm{H}}$ denotes Hermitian complex transpose of a matrix, $\sigma_{n}^{2}$ is the complex noise variance and $N T$ is the number of training blocks. The objective is to pick $\breve{\mathrm{H}}[\bar{n}, \bar{c}]$, which maximizes the likelihood function in (3.5) or minimize the kernel of (3.5).

The solution to the maximization of (3.5) to obtain the $\breve{\mathbf{H}}[\bar{n}, \bar{c}]$ gives:

$$
\breve{\mathbf{H}}[\bar{n}, \bar{c}]=\mathfrak{S}[\bar{n}]^{-1} \mathbf{B}[\bar{n}, \bar{c}]
$$

where

$$
\begin{aligned}
& \mathfrak{\Im}[\bar{n}]=\frac{1}{N T} \sum_{b=1}^{N T} \overrightarrow{\mathbf{A}}_{b}[\bar{n}] \overrightarrow{\mathbf{A}}_{b}[\bar{n}]^{\mathrm{H}} \\
& \mathbf{B}[\bar{n}, \bar{c}]=\frac{1}{N T} \sum_{b=1}^{N T} \overrightarrow{\mathbf{A}}_{b}[\bar{n}]^{\mathrm{H}} \breve{\mathbf{Y}}_{b}[\bar{n}, \bar{c}]
\end{aligned}
$$

where $\bar{n}=0,1, \ldots, T_{\mathrm{r}}-1$. Note that the size of $\breve{\mathbf{H}}$ is equal to the length of the short training sequence $\left(T_{r}\right)$ or the total number of pilots in one data block. In order to obtain the estimate for all the sub-carriers for $k$ th ICU i.e. $\hat{\mathbf{H}}_{k}^{0}$ (equal to data block length $B$, assuming $T_{r}<B$ ), 2x1D Wiener interpolation filters are used. 


\subsubsection{Orthogonal Training}

Second possible pilot coordination is to place the pilots orthogonally among the ICUs. Orthogonality between the short training blocks or pilots of different users eliminates inter-user interference. Orthogonality can be achieved by an etiquette-driven system in which only one ICU sends its short training block at a time or leaves those tones unused, which are being used for pilots by other ICUs. With two users, a well-known space-time coding known as Alamouti coding also serves this purpose. But the Alamouti precoding scheme is valid for only two users' case. For more than two users, we force them to be orthogonal with the help of a centralized control by the BS over the users' training timings or frequencies.

\subsubsection{Orthogonal Time Multiplexed Training (TMT-ortho)}

In orthogonal Time Multiplexed Training (TMT-ortho), at a certain time instant no other ICU transmits if one user is transmitting its short training block. On the reception of a short training block after FFT, (3.1) becomes:

$$
\breve{Y}_{m}[\bar{n}, \bar{c}]=H_{m}^{k}[\bar{n}, \bar{c}] \vec{A}^{k}[\bar{n}]+N_{m}[\bar{n}, \bar{c}]
$$

having no interference from the $K-1$ users. Hence the users' training blocks would remain orthogonal to each other and do not interfere. As every user follows the same etiquette, they all remain orthogonal during the training phase. TMT-ortho with an assumption of three users in the system, is depicted in Figure 3.2. ' $\mathrm{Tr}^{\prime}$ in the figure represents short pilot-carrying block.

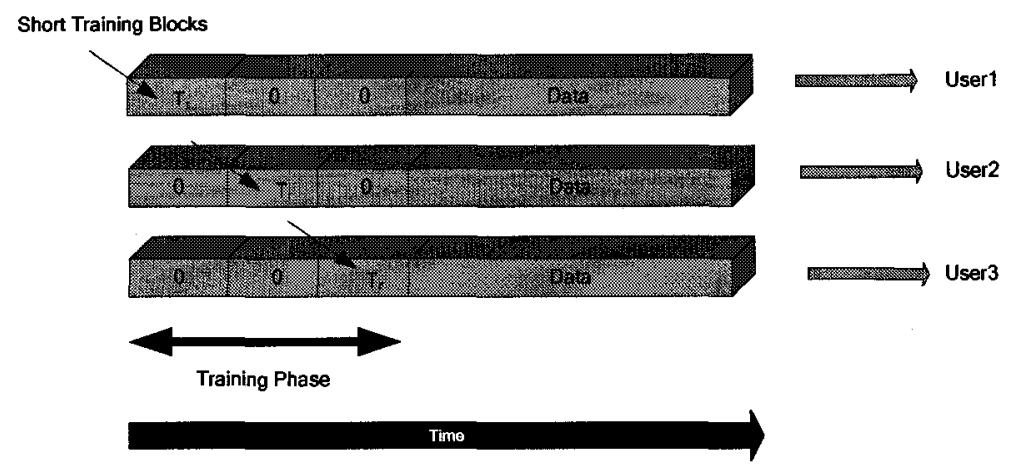

Fig. 3.2. Orthogonal Time Multiplexed Training (TMT-ortho with three users). 


\subsubsection{Orthogonal Frequency Multiplexed Training (FMT)}

In case of orthogonal FMT, we keep different ICUs' pilots orthogonal to each other. For example, pilots in three users' data blocks are shown in Figure 3.3 in which ' $T$ ' and ' $d$ ' denote the pilot and data symbols respectively. In orthogonal FMT, the pilots of $k$ th user are placed such that the other $K-1$ users should have zero at those specific tones.

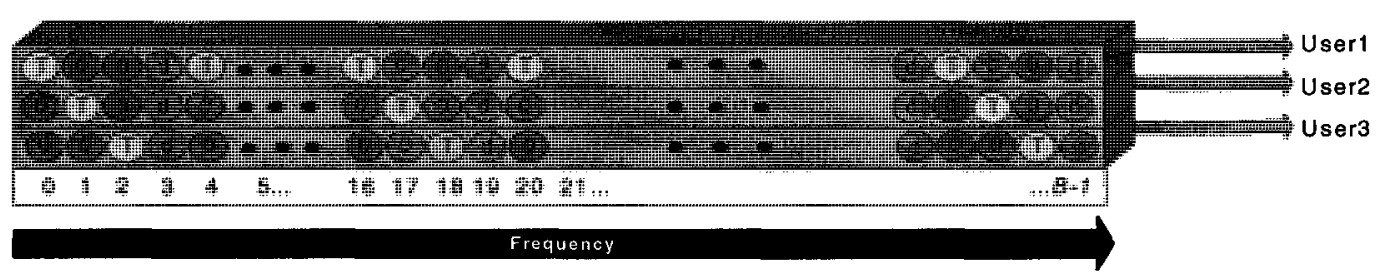

Fig. 3.3. Concept of Frequency Multiplexed Training (FMT) with three users.

\section{Multi-user Orthogonal pilot Signal Generation:}

Orthogonal frequency domain pilot distribution in different users is generated by using the concept described in [CBM04b], in which a Chu sequence for the first ICU $(k=1)$ is generated using (2.12) and rewritten for convenience (for even $T_{r}$ ):

$$
a^{1}(\bar{q})=\exp \left(\vec{\jmath} \pi \bar{q}^{2} / T_{r}\right)
$$

where $\bar{q}=0,1, \ldots, T_{r}-1$ and $\vec{z}$ represents prime number relative to $T_{r}$. A base-sequence is generated by repeating the Chu sequence generated in (3.9) $P_{r}$ times for $P_{r}=B / T_{r}$, where $B$ is the data block size and $T_{r}$ is the total number of pilots in a block. If we denote the Chu sequence generated by using (3.9) as:

$$
\dot{\mathbf{a}}^{1}=\left(a^{1}(0), a^{1}(1), \ldots, a^{1}\left(T_{r}-1\right)\right)
$$

the base sequence can be written as:

$$
\mathbf{a}^{1}=\left(\dot{\mathbf{a}}_{1}^{1}, \dot{\mathbf{a}}_{2}^{1}, \ldots, \dot{\mathbf{a}}_{P_{r}}^{1}\right)
$$

where $\mathbf{a}^{1} \in C^{1 \times B}$. FFT of $\mathbf{a}^{1}$ generates scattered pilots within the data block of the first user.

$$
\overrightarrow{\mathbf{A}}^{1}=\frac{1}{P_{r}} \mathbf{F}_{B}\left(\mathbf{a}^{1}\right)
$$

where $\mathbf{F}_{B}$ represents a $B$-point FFT. The result is a Frequency-Domain Orthogonal Signature Sequences (FDOSS) [CC00], or Interleaved Frequency Division Multiple 
Access (IFDMA) signal bearing FFT of the Chu sequence of the first user. Time domain base sequence of length $B$ is used to generate the other user's orthogonal sequences in frequency by multiplying the base sequence with an exponential term (phase rotation).

$$
a^{k}(\bar{q})=a^{1}(\bar{q}) \exp (j 2 k \pi(\bar{q} / B))
$$

for $k=1,2, \ldots, K$, and $\bar{q}=0,1, \ldots, B-1$. The FFT of these phase-rotated sequences gives the orthogonal pilots for all the other users in the system, given by:

$$
\overrightarrow{\mathbf{A}}^{k}=\frac{1}{P_{r}} \mathbf{F}_{B}\left(\mathbf{a}^{k}\right)
$$

Therefore in FMT, similar to orthogonal TMT, (3.1) becomes

$$
\breve{Y}_{m}[\bar{n}, \bar{c}]=H_{m}^{k}[\bar{n}, \bar{c}] \vec{A}^{k}[\bar{n}]+N_{m}[\bar{n}, \bar{c}]
$$

having no interference from the $K-1$ users.

\subsubsection{Pilot Overhead Definition}

For orthogonal ICUs the pilot overhead is defined as:

overbead $=\frac{(\# \text { of pilot }- \text { canying blocks per frame }) *(\# \text { of ICUs }) *(\# \text { of short training blocks }) *(\# \text { of pilots per ICU per training block })}{(\# \text { of blocks per frame }) *(\# \text { of symbols per block })}$

\subsubsection{Least Squares (LS) Multi-User CE for Orthogonal Training}

With Orthogonal TMT or Orthogonal FMT, the full LS joint estimation simplifies to a simple division of the received vector (array input) with the known training. If $\overrightarrow{\mathbf{A}}[\bar{n}]$ denote short training blocks in TMT or pilot sequence generated as described above in FMT, and $\breve{Y}[\bar{n}, \bar{c}]$ is the received signal during training phase in TMT or picked from every pilot location in case of FMT, then by simple element by element division on the received vector, we obtain the initial short estimates and (3.6) becomes:

$$
\breve{\mathbf{H}}[\bar{n}, \bar{c}]=\frac{\breve{\mathbf{Y}}[\bar{n}, \bar{c}]}{\breve{\mathbf{A}}[\bar{n}]}
$$

$\breve{\mathbf{Y}}[\bar{n}, \bar{c}]$ is the array input vector $\in \mathrm{C}^{M \times 1}$ for each pilot frequency $\bar{n}$, for $\bar{n}=0,1, \ldots, T_{r^{-}} 1$ and $\bar{c}$ represents the index for the pilot-carrying blocks in a frame. After obtaining the 
short frequency response, interpolation in frequency and time is carried out to obtain estimates of the whole time-frequency grid for each ICU.

\subsection{Comparison between Orthogonal and Overlapping}

\section{Training}

In this section, we present a set of simulation results illustrating different combinations of TMT and FMT training with different numbers of orthogonal or overlapping ICUs and receiver diversity. For the sake of brevity, we drop the term "ortho" with FMT. Therefore, wherever FMT is used, it represents orthogonal FMT. In case of TMT we still use TMT-ortho, TMT-Chu, TMT-Al or TMT-QPSK to differentiate between the type of sequence used and to show that they are orthogonal or not. Following conventions are used:

- TMT-ortho Orthogonal TMT.

- TMT-Chu Overlapping TMT with all users sending different Chu sequences at any time-frequency instance.

- TMT-QPSK Overlapping TMT with all users sending different random QPSK symbols at any time-frequency instance.

- TMT-Al Orthogonal TMT with space-time Alamouti coding (only valid for $K=2$ ).

- FMT or FMT-Chu Orthogonal FMT with Chu sequences used as pilots.

\subsubsection{Simulation Results}

\section{System Parameters and Spatial Distribution:}

System and simulation parameters are selected from a European WINNER project [Win02] and given in Table 3.1. 
Table 3.1. System Parameters

\begin{tabular}{|c|c|}
\hline FFT size & $X=512$ \\
\hline Used sub-carriers & $B=416$ \\
\hline Number of pilots & $T_{r}=104$ \\
\hline Channel coding (convolutional) & $(133,171)_{0}$ \\
\hline Modulation Scheme & QPSK \\
\hline Carrier frequency & $4.2 \mathrm{GHz}$ \\
\hline Signal BW & $16.25 \mathrm{MHz}$ \\
\hline Duplex mode & FDD \\
\hline Sub-carrier spacing & $39.0625 \mathrm{kHz}$ \\
\hline Maximum Doppler frequency & $194.4 \mathrm{~Hz}$ \\
\hline Up sampling factor & 10 \\
\hline Time domain windowing roll off factor & $23 \%$ \\
\hline Total symbol length & $28.8 \mu \mathrm{sec}$ \\
\hline Cyclic Prefix length & $3.2 \mu \mathrm{sec}$ \\
\hline Number of blocks/Code Frame & $F=12$ \\
\hline Number of users in the system & $K=1,2$ or 3 \\
\hline Number of receiving elements & $M=1,2$ or 3 \\
\hline Number of short training blocks $(N T)$ in case & Vary from case to case \\
of TMT & \\
\hline
\end{tabular}

The spatial distribution of the ICUs in the simulation assumes equi-distant (equal average received power) uplink users. Average Bit Error Rate (BER) performance of SC-FDE, with different pilot-multiplexing schemes described above, has been evaluated. A frequency selective time varying channel [Win01] with a vehicular speed of $50 \mathrm{~km} / \mathrm{hr}$ is used. The average PDP was given in Table 2.1. Average BER curves with Perfect Channel State Information (PCSI) are presented for reference purposes. QPSK modulation-based single carrier system is simulated for a range of received Signal to Noise Ratios (SNRs), each with 2000 random channel realizations. SNR is the signal to noise ratio per receive antenna element. A random interleaver and convolutional 1/2-rate encoder with a constraint length of 7 and code generator polynomial $[133,171]$ is used. Other simulation parameters are given in Table 3.1. In most of the results, number of receiving elements $(M)$ is kept equal to the number of ICUs $(K)$ unless otherwise stated. All users' SC-FDE frames are assumed to be perfectly synchronized. With TMT, to the first and the last block of each frame is added two short training blocks containing a $\mathrm{Chu}$ sequence of 104 pilot symbols. The resulting pilot waveform is therefore uniform in amplitude and spectrum. With FMT, there are 104 pilots spread over the first, second, 
eleventh and last block of the code frame. Note that for equal pilot overhead in TMT and FMT, we send more pilot-carrying blocks in FMT to compensate the cases of NT>1 with TMT.

\subsubsection{Comparison between TMT and FMT}

Two ICUs with two antenna elements at the BS is compared in Figure 3.4. With two users, there are three possible training schemes with which we maintain the orthogonality between them. They are Alamouti Space Time Transmit Diversity (STTD), TMT-ortho and FMT. We compare the average BER performances with all of these orthogonal pilot options. Initial channel estimate for each user to each receiving antenna is made by using the LS estimation and 2x1D Wiener interpolation described in Sections 2.7.5 and 2.7.6.3. From channel estimates frequency domain LE array coefficients $\hat{\mathbf{W}}[n]$ as given in (2.11) is obtained, and the equalizer output for each user is passed to a decoder, yielding data symbol decisions.

Orthogonal FMT shows the same performance as with TMT-ortho and TMT-Al with equal amount of pilot overhead, with a difference of around 2.3dB from the PCSI. TMTChu is the non-orthogonal counter part with same pilot overhead. It is observed that with time averaging over three short training blocks (same overhead as with orthogonal pilots), the performance of the overlapping pilots is $0.8 \mathrm{~dB}$ worse than the orthogonal counter parts. Figure 3.4 also suggests that the TMT-Chu could perform slightly better at the cost of additional training blocks but still worse than the orthogonal pilot arrangements. TMT-QPSK is the case of overlapping training with pilots consisting of random QPSK symbols. 


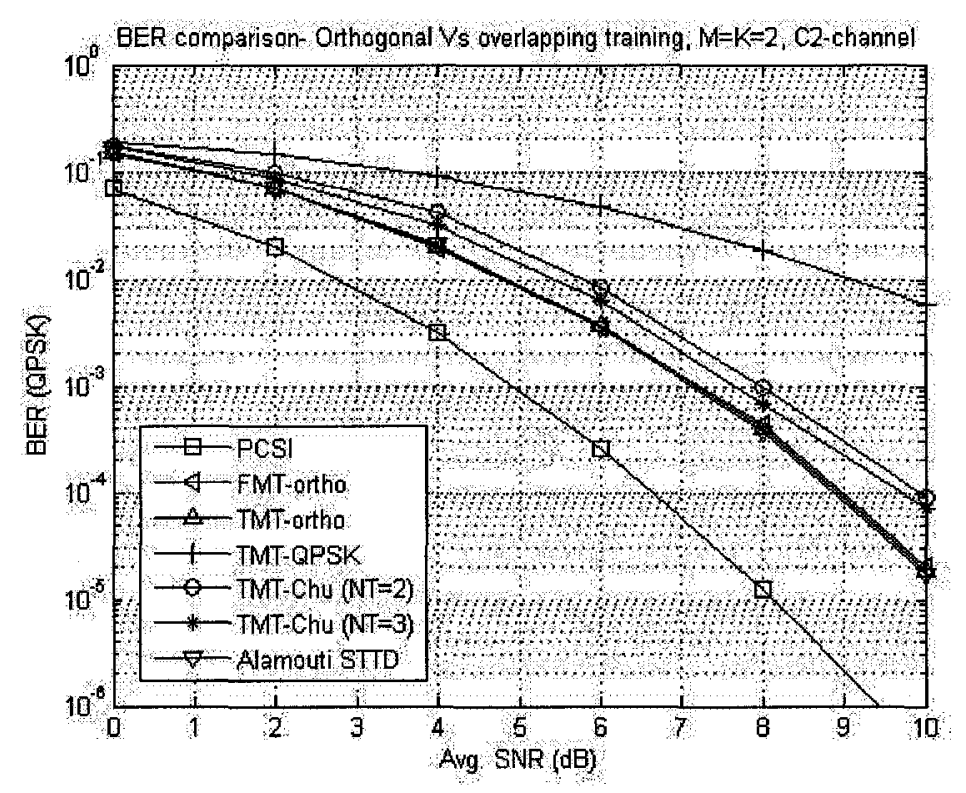

Fig. 3.4. Coded average BER performance, $M=K=2, N T=2$ and 3, LE - Performance of orthogonal FMT Vs Overlapping TMT with QPSK random data symbols as training (Perfect Channel State Information (PCSI) curve shown for reference).

In order to overcome the intra-cell interference, if orthogonality between ICUs during their training phase is assured, then better performance is achievable than with nonorthogonal training with equal amount of overhead. Moreover, it is shown that the way in which we multiplex the training either in time or frequency does not affect the performance in time-variant channels as long as the orthogonality is maintained. Therefore, intra-cell interference could be managed by orthogonal training either in time or frequency.

In Figure 3.4, the SNR gap between PCSI BER performance and non-PCSI performance for $\mathrm{LE}$ is around $2.3 \mathrm{~dB}$ which is around $0.8 \mathrm{~dB}$ more than the SNR degradation observed in the Single-Input Single-Output (SISO) case [LFD08] and also in Figures 3.6 and 3.7. This CE degradation is caused by the effect of the multi-user SDMA interference. Better $\mathrm{CE}$ algorithms like decision feedback iterative $\mathrm{CE}$ described in later chapters are needed to suppress these effects. 


\subsubsection{Comparison between Interpolation Choices and Complexities}

Figures 3.5-3.7 show comparison between a few of the possible combinations of different interpolation schemes and their complexities. One antenna element $(M=1)$ and one user $(K=1)$ are considered. Figures 3.5 and 3.6 show the average MSE at the equalizer output and the corresponding average BER curves with different interpolation combination choices. The legend FFT/Linear represents FFT-interpolation described in Section 2.7.6.2 in the frequency axis with a Linear Interpolation (LI) in the time axis. Similarly, other legends can be interpreted. Superior results suggest the use of Wiener filter in both time and frequency axes $(2 \times 1 \mathrm{D}$ filter).

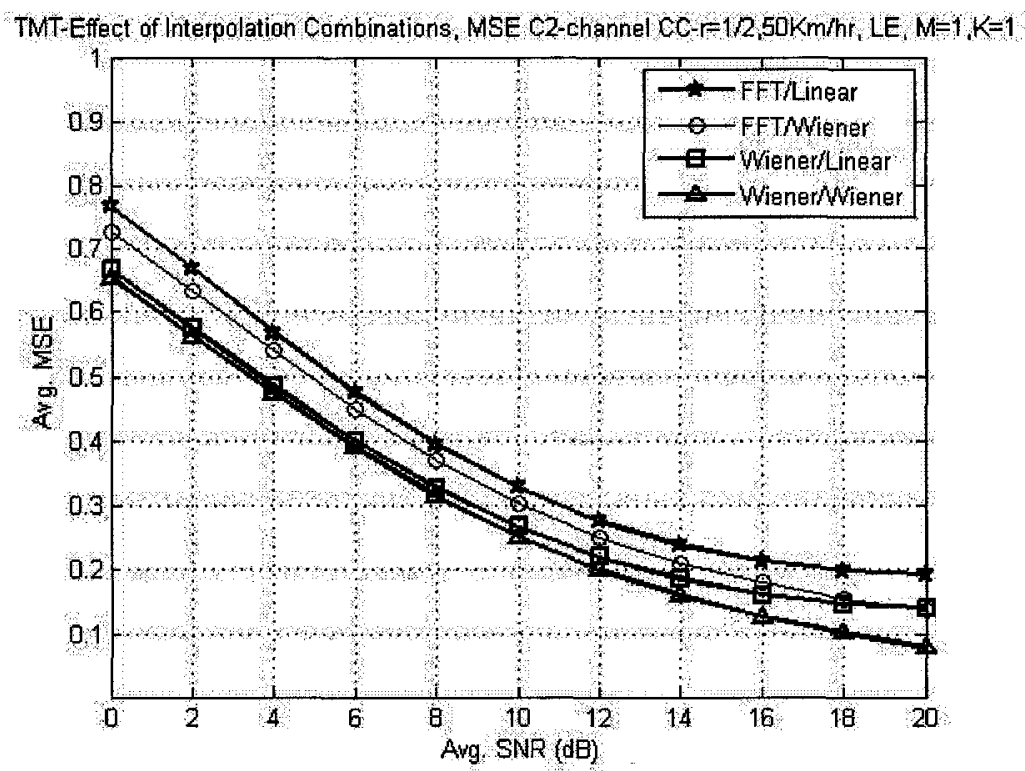

Fig. 3.5. Comparison of interpolation choices (FMT, $M=K=1$ ) in terms of average MSE. 


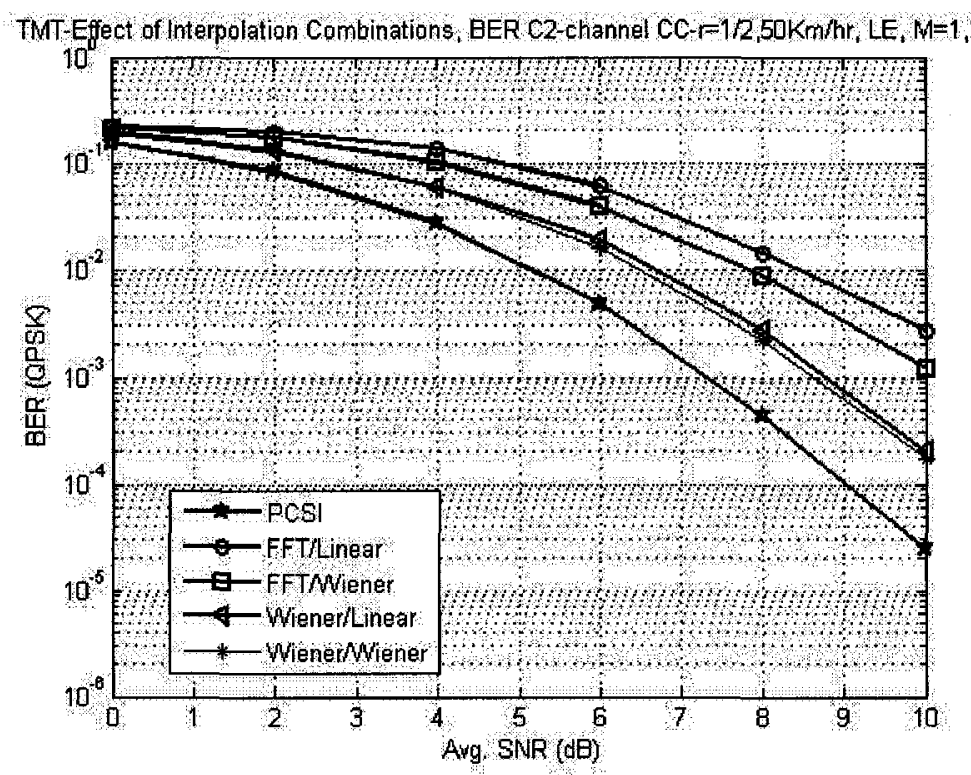

Fig. 3.6. Comparison of interpolation choices (FMT, $M=K=1$ )- coded average BER.

A concern associated with the $2 \times 1 \mathrm{D}$ interpolation is the computational complexity, which increases with the selected window size in the time and frequency. Three different window sizes are compared. Average BER with these three window sizes are shown in Figure 3.7. It can be seen that we may obtain slightly higher gain at higher SNR values with more complex interpolation parameters. $13 \times 13$ in the legend shows the window size of $13\left(Q_{n}=13\right)$.

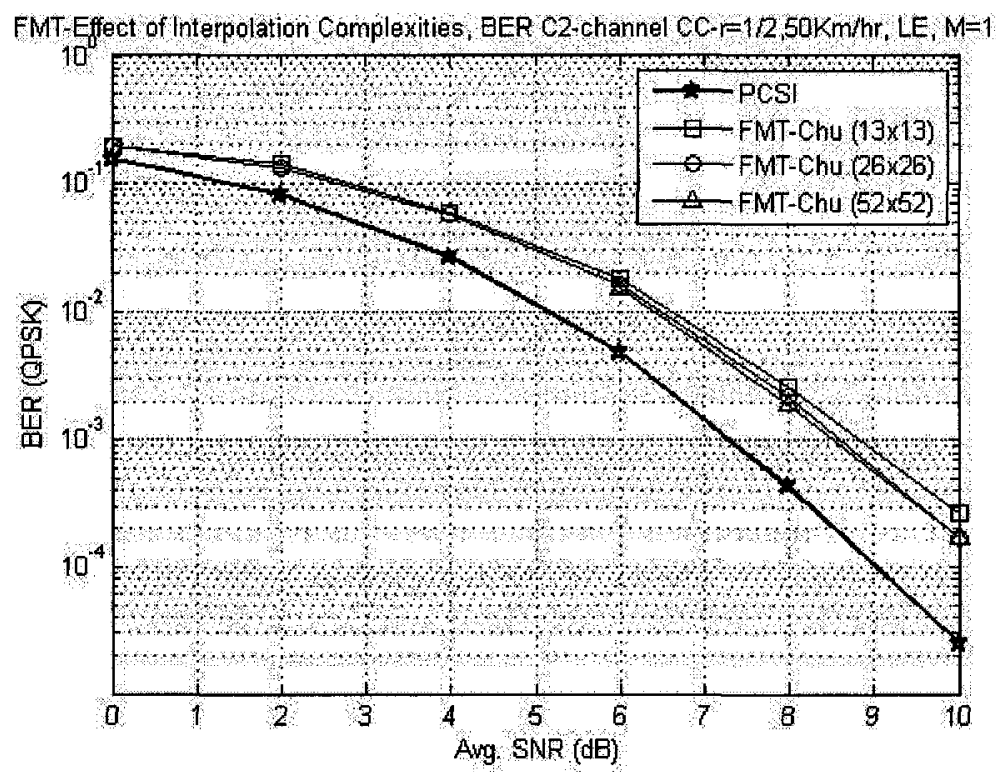

Fig. 3.7. Comparison of interpolation complexities (FMT, $M=K=1$ )- coded average BER. 


\subsubsection{Time Averaging in TMT}

In TMT an improvement via time averaging of short training sequences is possible. We send more short training blocks, assuming that they are short enough that the channel does not change much during the transmission. At the receiver, the averaging of such training blocks gives us better channel estimates by suppressing the effects of noise at the cost of additional overhead. In the following simulations, contiguous short training blocks ( 1 or 2$)$ at the beginning of the first and last data block in each code frame are used. With a single link, the same Chu-sequence-based training can be used in all short blocks while in multi-user case different sequences per user are sent. A more detailed description of time averaging is given in Chapter 4 where we deal with the Out of Cell Interferers (OCIs). Figure 3.8 shows an improvement of around $0.5 \mathrm{~dB}$ in average BER by sending two training blocks compared to the case with no time averaging. Here we see a bit more improvement with time averaging compared to the improvement shown in Figure 3.4 (from $N T=2$ to $N T=3$ ) due to the multi-user interference.

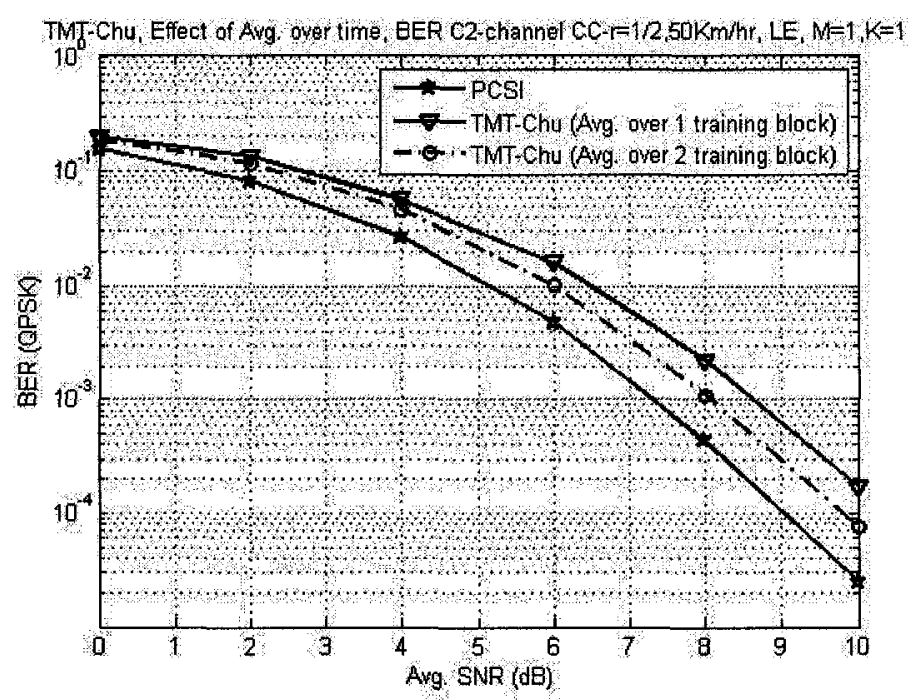

Fig. 3.8. Effect of time averaging on coded average BER (TMT-Chu), $N T=1$ and 2, LE.

\subsubsection{CE and Vehicular Speeds}

Higher mobility affects the CE process. From the pilot multiplexing point of view, a concern is their performances in the presence of higher vehicular speeds. It is shown 
through simulations that the higher Doppler does not affect the multiplexing way differently. This is shown in Figure 3.9.

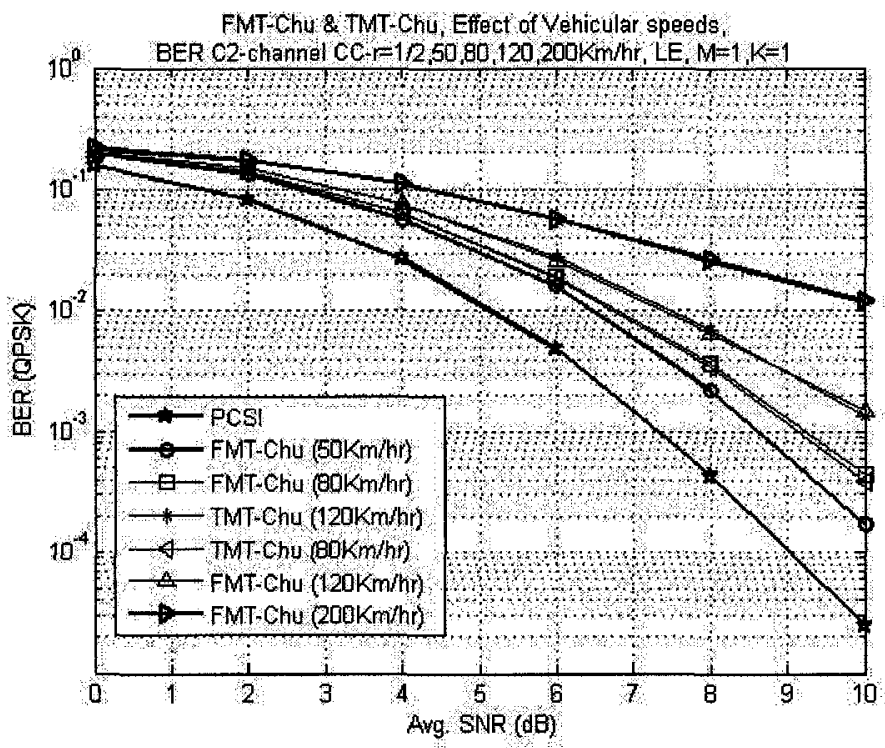

Fig. 3.9. Coded average BER performance, effect of different Doppler on pilot multiplexing and channel estimation $(M=K=1)$.

\subsection{BER Analysis with Channel Estimation}

In this section we present an analysis for the average MSE at the equalizer output and the CE error. By using the computed MSE, we calculate the average BER by using a wellknown Gaussian Approximation (GA) with PCSI (known CFRs) as well as with the CE errors. It is shown that the approximation works well in the presence of intra-cell interference by comparing the analytical results with the simulation results.

With PCSI the average MMSE at the equalizer output is given in (2.10) and given below for convenience:

$$
J_{\min }=\frac{1}{B^{*} K} \sum_{n=0}^{B-1} \operatorname{tr}\left[\left(\mathbf{W}[n]^{\mathrm{H}} \mathbf{H}[n]-\mathbf{I}_{K}\right)\left(\mathbf{W}[n]^{\mathrm{H}} \mathbf{H}[n]-\mathbf{I}_{K}\right)^{\mathrm{H}}+\sigma_{n}^{2} \mathbf{W}[n] \mathbf{W}[n]^{\mathrm{H}}\right]
$$

where $t r$ is the trace of a matrix, $\sigma_{n}^{2}$ is the noise variance, $\mathbf{I}_{K}$ is an identity matrix of size $K, \mathbf{H}[n] \in \mathbb{C}^{M \times K}$ is the CFR matrix, ${ }^{\mathrm{H}}$ denotes Hermitian transpose and the antenna array weighing coefficients of the LE, $\mathbb{W}[n] \in C^{M x K}$ in case of Perfect Channel State 
Information (PCSI) are given as $\mathbf{W}[n]=\left[\mathbf{H}[n] \mathbf{H}[n]^{\mathrm{H}}+\sigma_{n}^{2} \mathbf{I}_{M}\right]^{-1} \mathbf{H}[n], n=0,1, \ldots, B-1$. With the assumption that the residual error at the LE output is Gaussian with zero mean and variance equals to $J_{\min }$, the BER performance of an uncoded QPSK system can be written as:

$$
B E R=Q\left(\sqrt{\frac{1-J_{\min }}{J_{\min }}}\right)=\frac{1}{2} \operatorname{erfc}\left(\sqrt{\left(\frac{1-J_{\min }}{J_{\min }}\right) / 2}\right)
$$

where $Q(x)$ is Gaussian probability integral [Hay02] and defined as:

$$
Q(x)=\frac{1}{\sqrt{2 \pi}} \int_{x}^{\infty} e^{-\frac{y^{2}}{2}} d y
$$

The kernel of the (3.18) represents the Signal to Interference plus Noise Ratio (SINR).

Figure 3.10 shows the effectiveness of the approximation (3.18) with single user and single element scenario $(M=K=1)$. PCSI average BER result with the approximation using (3.18) perfectly matches the simulation curve.

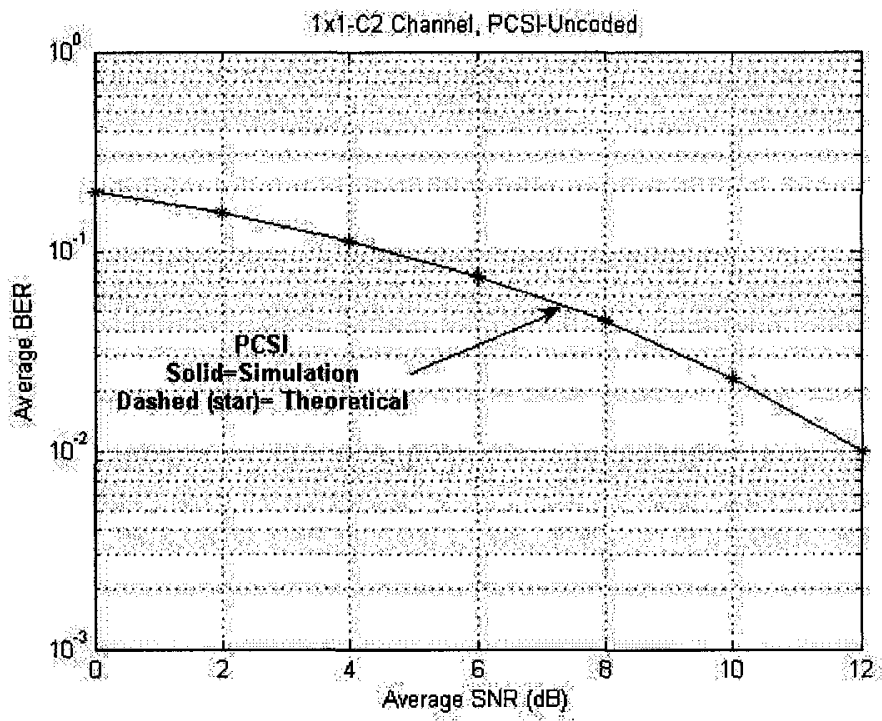

Fig. 3.10. Average uncoded BER, Analytical Vs Simulation, $M=K=1$, LE with PCSI.

\subsubsection{Analysis of CE Error in Multi-User Scenario}

Channel estimation error in general includes the effect of noise and interpolation errors. An analysis on parameterization of the estimation error is presented in [Gun04]. In multi- 
user systems CE error is also affected by the multi-user interference. If the estimated CFR at antenna element $m$ for $k$ th user at $n$th frequency is denoted by $\hat{H}_{m}^{k}[n]$, then the decision variable of the transmitted symbol $S^{k}[n]$ is given by:

$$
\hat{S}^{k}[n]=\frac{Y_{m}[n]}{\hat{H}_{m}^{k}[n]}=\frac{H_{m}^{k}[n] S^{k}[n]+Z_{m}^{\prime \prime}[n]}{\hat{H}_{m}^{k}[n]}
$$

where $Y_{m}[n]$ represents the element of the array input at $m$ th antenna at $n$th sub-carrier and $Z_{m}^{\prime \prime}[n]$ includes the effect of noise and multi-user interference. The channel estimation error for $k$ th user at $n$th frequency and at $m$ th antenna element is assumed to be a Gaussian random variable and is given by:

$$
x_{m}^{k}[n]=H_{m}^{k}[n]-\hat{H}_{m}^{k}[n]
$$

where $H_{m}^{k}[n]$ represents the actual CFR. The objective here is to analytically estimate the BER performance of an uncoded QPSK system in such environments, given the average MSE of the multi-user channel estimates. The BER can be found by replacing the MMSE $J_{\min }$ in (3.18) with the MSE with CE errors $J_{\text {est }}$. Such MSE is the sum of the MMSE and the access MSE caused by the CE errors. The MSE averaged over all the ICUs can be computed as:

$$
J_{\text {est }}=\frac{1}{B^{*} K} \sum_{n=0}^{B-1} \operatorname{tr}\left[\left(\hat{\mathbf{W}}[n]^{\mathrm{H}} \mathbf{H}[n]-\mathbf{I}_{K}\right)\left(\hat{\mathbf{W}}[n]^{\mathrm{H}} \mathbf{H}[n]-\mathbf{I}_{K}\right)^{\mathrm{H}}+\sigma_{n}^{2} \hat{\mathbf{W}}[n] \hat{\mathbf{W}}[n]^{\mathrm{H}}\right]
$$

We call this method semi-analytical approach. Using (3.22) and the actual equalizer output with the perfect and the estimated CFRs of the $M=K=2$ link, histograms and corresponding Cumulative Distribution Functions (CDF) are plotted in Figures 3.11 and 3.12 showing the validity of the Gaussian assumption. Figure 3.11 assumes CFR perfectly known (PCSI) while in Figure 3.12 CE errors are also included. 


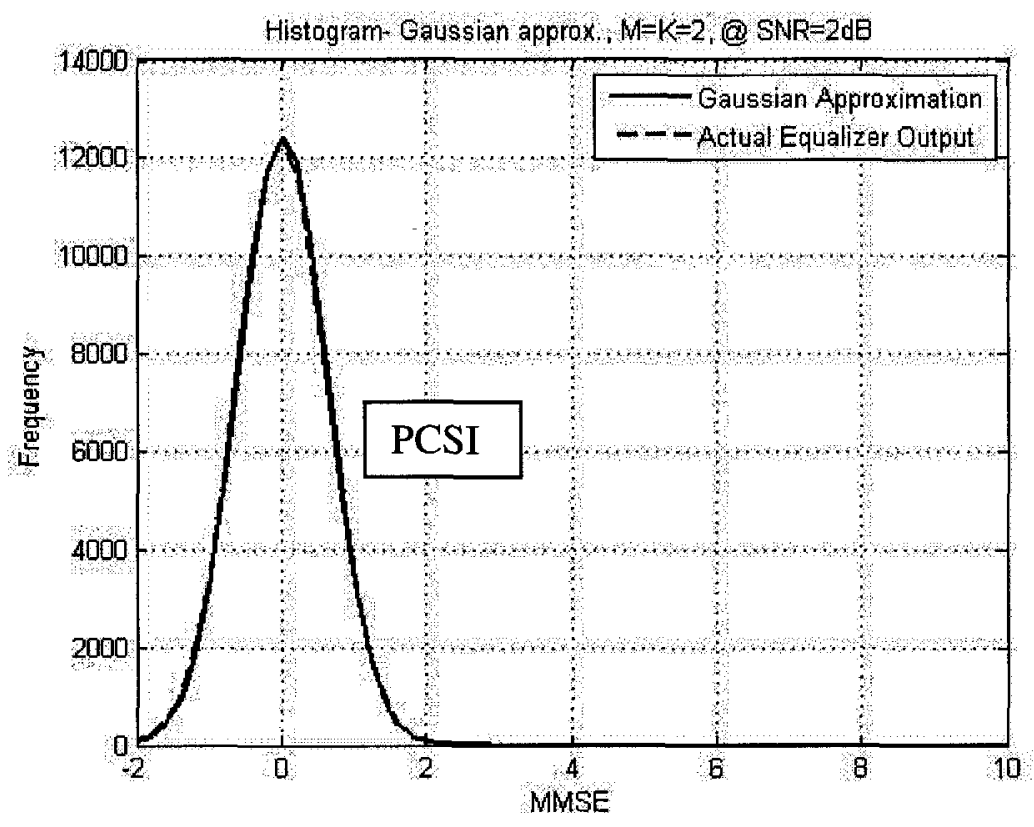

a)

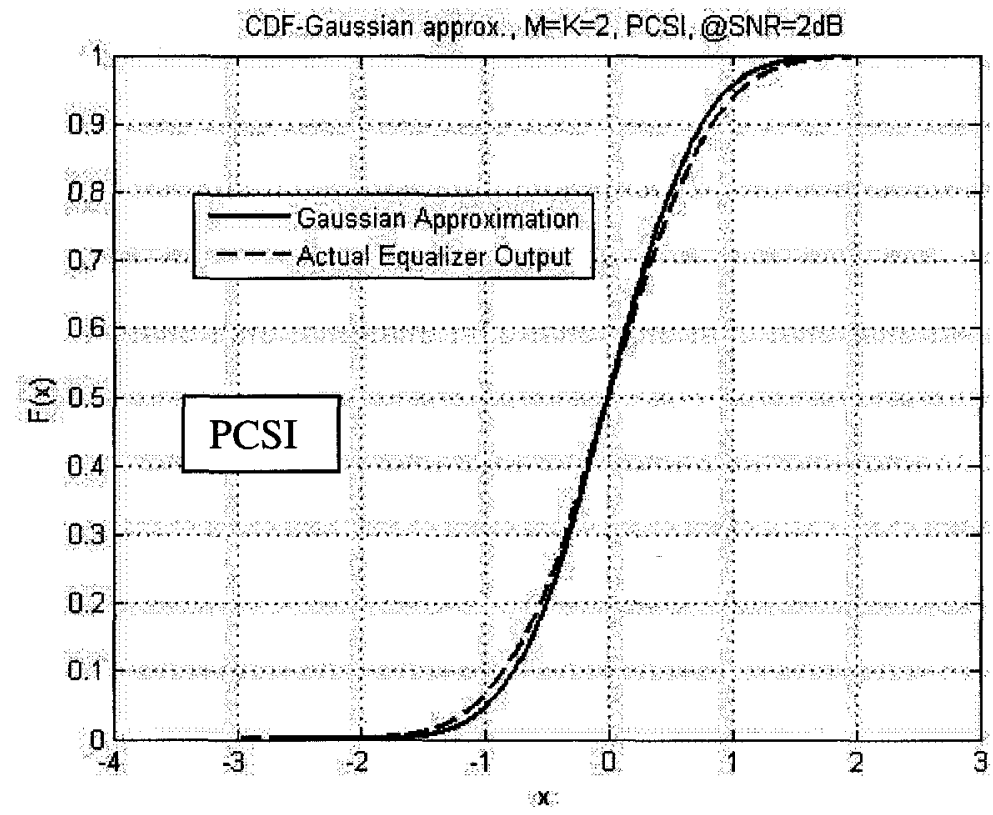

b)

Fig. 3.11. a) Histogram showing the validity of the Gaussian approximation of the equalizer output with no channel estimation errors (PCSI)- $M=K=2$, Uncoded-LE. b)

Corresponding CDF. 


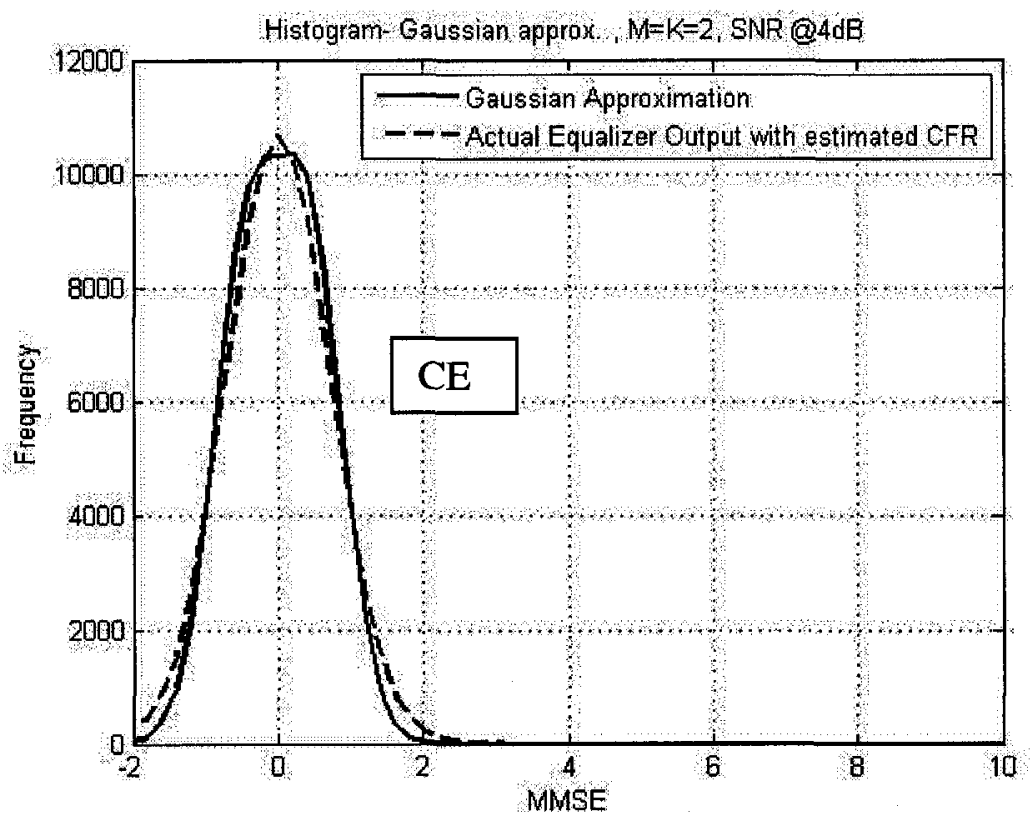

a)

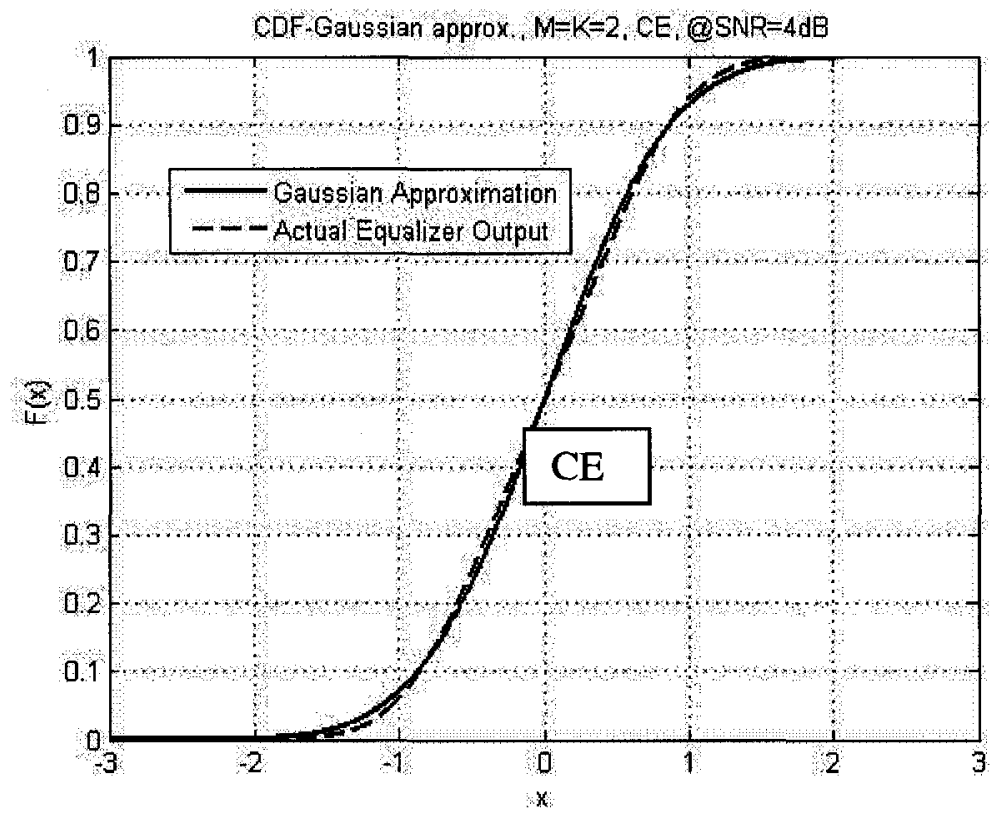

b)

Fig. 3.12. a) Histogram showing the validity of the Gaussian approximation of the equalizer output with channel estimation errors- $M=K=2$, Uncoded-LE. b) Corresponding CDF. 


\subsubsection{Mean Squared Error (MSE) Analysis}

Another way of finding the average MSE typically when using the indirect adaptation involve multi-user $\mathrm{CE}$ error. We call this method indirect adaptation-approach. The estimated equalizer coefficients are multiplied with the array input thusly:

$$
\mathbf{V}[n]=\hat{\mathbf{W}}[n]^{\mathrm{H}} \mathbf{Y}[n]
$$

where $\mathrm{V}[n] \in \mathrm{C}^{K \times 1}$. Equalizer output samples are given by:

$$
\mathbf{v}(i)=\mathbf{F}_{B}^{-1}(\mathrm{~V}[n])
$$

The CE error at frequency $n$ can be written as:

$$
x[n]=\mathbf{H}[n]-\hat{\mathbf{H}}[n]
$$

where, $\mathbf{H}[n] \in C^{M \times K}, \hat{\mathbf{H}}[n] \in \mathbb{C}^{M \times K}$ and $x[n] \in C^{M \times K}$

Using (3.22), substituting $\mathbf{H}[n]$ from (3.25) and the estimated weighing coefficients from (2.11), the MSE at the equalizer output can be written as:

$$
\begin{aligned}
& J_{\text {est }}=\frac{1}{B^{*} K} \sum_{n=0}^{B-1} \operatorname{tr}\left[\begin{array}{l}
{\left[(\hat{\mathbf{H}}[n]-x[n])^{\mathrm{H}}\left[\hat{\mathrm{H}}[n] \hat{\mathrm{H}}[n]^{\mathrm{H}}+\sigma_{n}^{2} \mathbf{I}_{M}\right]^{-1} \hat{\mathbf{H}}[n]-\mathbf{I}_{K}\right\rfloor} \\
{\left[(\hat{\mathbf{H}}[n]-x[n])^{\mathrm{H}}\left[\hat{\mathrm{H}}[n] \hat{\mathrm{H}}[n]^{\mathrm{H}}+\sigma_{n}^{2} \mathbf{I}_{M}\right]^{-1} \hat{\mathbf{H}}[n]-\mathbf{I}_{K}\right]^{\mathrm{H}}+} \\
\left.\sigma_{n}^{2}\left[\left[\hat{\mathbf{H}}[n] \hat{\mathbf{H}}[n]^{\mathrm{H}}+\sigma_{n}^{2} \mathbf{I}_{M}\right]^{-1} \hat{\mathbf{H}}[n]\right]\left[\hat{\mathbf{H}}[n] \hat{\mathbf{H}}[n]^{\mathrm{H}}+\sigma_{n}^{2} \mathbf{I}_{M}\right]^{-1} \hat{\mathbf{H}}[n]\right]^{\mathrm{H}}
\end{array}\right] \\
& J_{\text {est }}=\frac{1}{B^{*} K} \sum_{n=0}^{B-1} t r\left[\|\left[\begin{array}{l}
\left.\| \hat{\mathbf{H}}[n]^{\mathrm{H}}\left[\hat{\mathbf{H}}[n] \hat{\mathbf{H}}[n]^{\mathrm{H}}+\sigma_{n}^{2} \mathbf{I}_{M}\right]^{-1} \hat{\mathbf{H}}[n]-\mathbf{I}_{K}\right]-\varkappa[n]^{H}\left[\hat{\mathbf{H}}[n] \hat{\mathbf{H}}[n]^{\mathrm{H}}+\sigma_{n}^{2} \mathbf{I}_{M}\right]^{-1} \hat{\mathbf{H}}[n] \|^{2}+ \\
\sigma_{n}^{2}\left[\left[\hat{\mathbf{H}}[n] \hat{\mathbf{H}}[n]^{\mathrm{H}}+\sigma_{n}^{2} \mathbf{I}_{M}\right]^{-1} \hat{\mathbf{H}}[n]\left[\hat{\mathbf{H}}[n] \hat{\mathbf{H}}[n]^{\mathrm{H}}+\sigma_{n}^{2} \mathbf{I}_{M}\right]^{-1} \hat{\mathbf{H}}[n]\right]^{\mathrm{H}}
\end{array}\right]\right. \\
& J_{\text {est }}=\frac{1}{B^{*} K} \sum_{n=0}^{B-1} \operatorname{tr}\left[\begin{array}{l}
\left.\| \begin{array}{l}
\hat{\mathbf{H}}[n]^{\mathrm{H}}\left[\hat{\mathbf{H}}[n] \mathbf{H}[n]^{\mathrm{H}}+\sigma_{n}^{2} \mathbf{I}_{M}\right]^{-1} \hat{\mathbf{H}}[n]-\mathbf{I}_{K}\left\|^{2}+\right\| \varkappa[n]^{H}\left[\hat{\mathbf{H}}[n] \hat{\mathbf{H}}[n]^{\mathrm{H}}+\sigma_{n}^{2} \mathbf{I}_{M}\right]^{-1} \hat{\mathbf{H}}[n] \|^{2}- \\
\left.\left.\left(\hat{\mathbf{H}}[n]^{\mathrm{H}}[\hat{\mathbf{H}}[n]]^{\mathrm{H}}\left[\hat{\mathbf{H}}[n]^{\mathrm{H}}+\sigma_{n}^{2} \mathbf{I}_{M}\right]^{-1} \hat{\mathbf{H}}[n]-\mathbf{I}_{K}\right)^{H}[n]^{\mathrm{H}}+\sigma_{n}^{2} \mathbf{I}_{M}\right]^{-1} \hat{\mathbf{H}}[n]-\mathbf{I}_{K}\right)
\end{array}\right\} \\
+\sigma_{n}^{2}\left[\begin{array}{l}
\left.\left[\hat{\mathbf{H}}[n] \hat{\mathbf{H}}[n]^{\mathrm{H}}+\sigma_{n}^{2} \mathbf{I}_{M}\right]^{-1} \hat{\mathbf{H}}[n]\left[\hat{\mathbf{H}}[n] \hat{\mathbf{H}}[n]^{\mathrm{H}}+\sigma_{n}^{2} \mathbf{I}_{M}\right]^{-1} \hat{\mathbf{H}}[n]\right]^{\mathrm{H}}
\end{array}\right.
\end{array}\right.
\end{aligned}
$$

By making the following assumptions

- $\left[\hat{\mathrm{H}}[n] \hat{\mathrm{H}}[n]^{\mathrm{H}}+\sigma_{n}^{2} \mathbf{I}_{M}\right]^{-1}=\left[\mathrm{H}[n] \mathrm{H}[n]^{\mathrm{H}}+\sigma_{n}^{2} \mathbf{I}_{M}\right]^{-1}$

- $\hat{\mathrm{H}}[n]^{\mathrm{H}}\left[\hat{\mathrm{H}}[n] \hat{\mathrm{H}}[n]^{\mathrm{H}}+\sigma_{n}^{2} \mathbf{I}_{M}\right]^{-1} \hat{\mathrm{H}}[n]=\mathbf{H}[n]^{\mathrm{H}}\left[\mathbf{H}[n] \mathbf{H}[n]^{\mathrm{H}}+\sigma_{n}^{2} \mathbf{I}_{M}\right]^{-1} \mathbf{H}[n]$ 
- $E\left[x[n]^{H} \hat{\mathbf{H}}[n]\right]=0$, orthogonality principle [Hay02]

- $\left.\left.E\|\| \mu[n]\right|^{2}\right]=\sigma_{x}^{2}$ (The MSE of the channel estimates at different frequencies are constant and equal to the variance $\sigma_{x}^{2}$ ).

(3.28) can be written as:

$$
J_{\text {est }}=\frac{1}{B^{*} K} \sum_{n=0}^{B-1} \operatorname{tr}\left(\begin{array}{l}
\sigma_{n}^{2}\left[\mathbf{H}[n] \mathbf{H}[n]^{\mathrm{H}}+\sigma_{n}^{2} \mathbf{I}_{M}\right]^{-1}+\sigma_{x}^{2} \mathbf{H}[n]^{\mathrm{H}}\left[\mathbf{H}[n] \mathbf{H}[n]^{\mathrm{H}}+\sigma_{n}^{2} \mathbf{I}_{M}\right]^{-1} \\
*\left[\mathbf{H}[n] \mathbf{H}[n]^{\mathrm{H}}+\sigma_{n}^{2} \mathbf{I}_{M}\right]^{-1} \mathbf{H}[n]
\end{array}\right)
$$

In (3.29) the first term represents the MMSE with PCSI while the second term is the excess MSE caused by the CE error.

\subsubsection{Comparison of Analytical and Simulation Results}

In this section we compare the simulation results with $2 \times 2(M=K=2)$ and $3 \times 3(M=K=3)$ cases with the two Gaussian approximation-based analytical approaches presented above. Simulations are carried out with uncoded, LE and $2000 \mathrm{C} 2$-channel realizations. The average BER comparison is shown in Figures 3.13 and 3.14 with $2 \times 2$ and $3 \times 3$ cases respectively. In the legend, 'IA Approach' means indirect approach given by (3.29), while 'Semi-analytical Approach' is given by (3.22). The figures illustrate the validity of the Gaussian assumption for the CE errors in a system with interference. It should be noted that with $\mathrm{CE}$ errors although the analytical approaches are not very tight, they are reasonably close to the simulation results. Therefore the MSE analysis presented above can replace the LE-based cumbersome simulation process. 


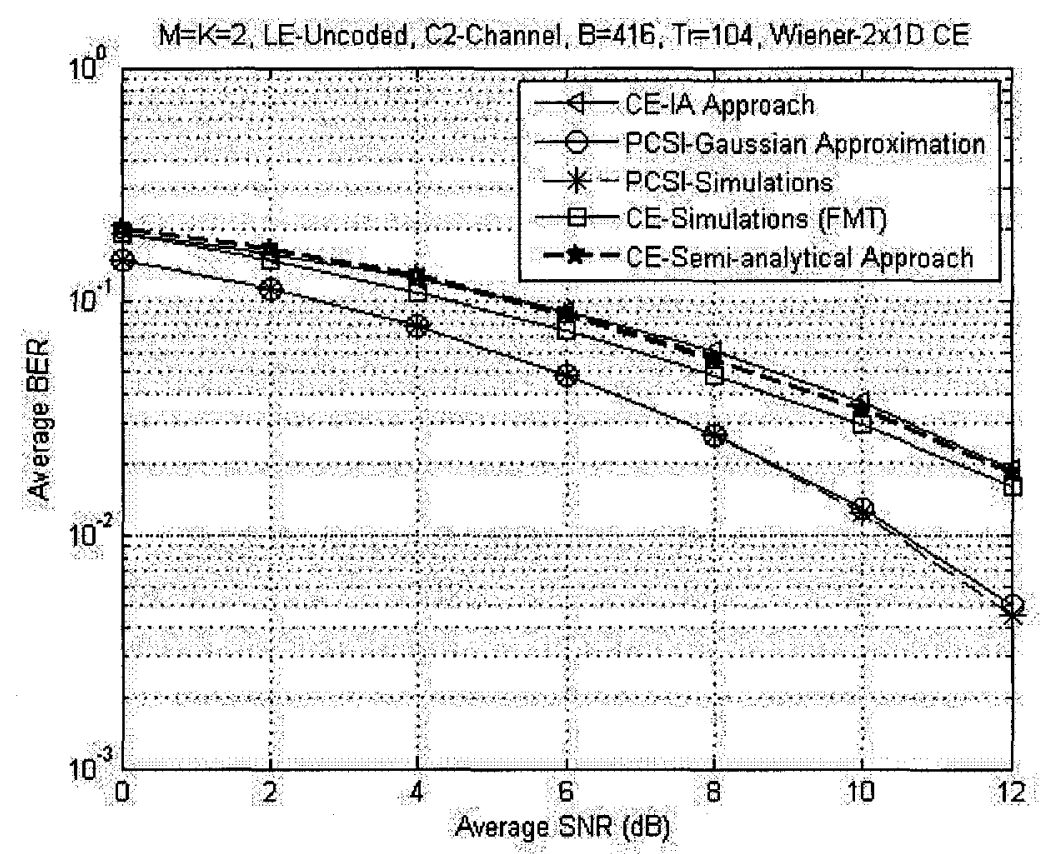

Fig. 3.13. Average BER-Gaussian approximation of the equalizer output with channel estimation errors and PCSI- $M=K=2$, Uncoded-LE.

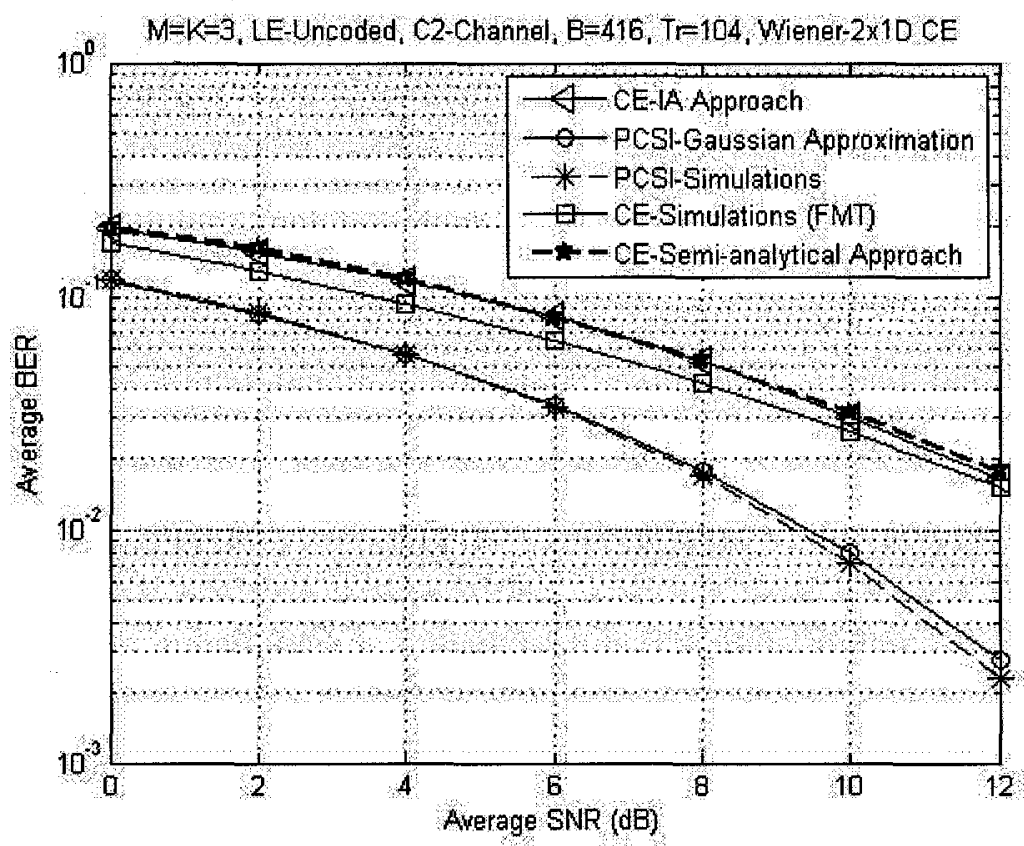

Fig. 3.14. Average BER-Gaussian approximation of the equalizer output with channel estimation errors and PCSI- $M=K=3$, Uncoded-LE. 


\subsection{Summary}

In this chapter, the issue of CE with intra-cell interference due to multiple In-Cell Users (ICUs) in SC-FDE system has been addressed. Several techniques and performance measures were tested, such as optimal training sequences and their placement, different methods of interpolation, LP filtration to suppress the effects of noise, effect of time averaging on the $\mathrm{CE}$, effect of mobility and receiver diversity. Analytical approaches for computing uncoded BER with MIMO channels are also presented. There are two main findings in the chapter. First, it was shown that with the multi-user SC-FDE system, an arrangement of time-multiplexed pilots gives the same performance as an equivalent arrangement of frequency multiplexed pilots. Secondly, it was shown that the channel estimates in the presence of the inter-user interference can be improved by keeping their training phases orthogonal to each other. Hence we can have equally good performance with orthogonal training either with TMT or FMT to mitigate the effects of inter-user interference in SC-FDE, SDMA system. 


\section{Chapter 4}

\section{Inter-Cell Interference in SC-FDE SDMA}

\section{Systems}

\subsection{Introduction}

Using SC-FDE in an interference-limited multi-cellular environment is relatively new. It is therefore desirable to obtain a better knowledge of how SC-FDE system performs in a cellular environment in the presence of inter-cell interference. In the previous chapter, it is shown that the channel estimates in the presence of intra-cell interference are improved by making the training of the In-Cell Users (ICUs) mutually orthogonal to each other. In this chapter, we extend our discussion of CE via interference avoidance with multiple InCell co-channel Users (ICUs) in the presence of Out of Cell Interferers (OCIs). When users from different cells are present on the same sub-carrier during a given symbol interval, the resulting signal at each receive antenna element of the BS is a result of a superposition of several co-channel uplink ICUs and OCIs. This chapter elaborates different forms of possible compensations and training arrangements that can be used to suppress the effects of OCIs, on the CE process of the desired ICUs.

\subsection{Signal \& System Model with OCIs}

This section presents an extension to the signal model presented in Chapter 2 with the inclusion of OCIs. The data to be transmitted by each in-cell and out of cell user is coded, interleaved and passed to a mapping unit, which uses QPSK modulation. Perfect synchronization between the ICUs and OCIs and full control over the selection of the training sequences for all the users at any time-frequency instance is assumed. This control is achieved by the coordination between the BSs of the interfering cells. 
At the BS, processing the signal vectors received at the array separates streams of the $K$ ICUs. Also, processing is carried out for $P$ OCIs, if the BS knows the training sequences of the OCIs. Each user's coded data symbols are organized into blocks of length $B$ in the same way that is presented in the signal model in Chapter 2. The resulting sequences from the users are transmitted through $K+P$ channel impulse responses. At the receiver a composite signal is received, after $X$-point FFT, we have

$$
\mathbf{Y}=\mathbf{F}_{X}(\mathbf{y})
$$

where $\mathbf{Y}\left[n_{1}\right]=\left[Y_{1}\left[n_{1}\right] \ldots Y_{M}\left[n_{1}\right]\right]^{\mathrm{T}}$ where $n_{1}=0,1, \ldots, X-1, n_{1}$ is the sub-carrier index and ${ }^{\mathrm{T}}$ in the superscript denotes transpose. $\mathbf{Y}\left[n_{1}\right]$ contains superposition of all ICUs and OCIs. The cyclic convolution is equivalent to the multiplication in the frequency domain; i.e., we obtain

$$
\mathbf{Y}\left[n_{1}\right]=\mathbf{H}\left[n_{1}\right] \mathbf{S}\left[n_{1}\right]+\mathbf{N}\left[n_{1}\right]
$$

where, $\quad \mathrm{Y}\left[n_{1}\right]=\left[\mathrm{Y}_{1}\left[n_{1}\right], \mathrm{Y}_{2}\left[n_{1}\right], \ldots, \mathrm{Y}_{M}\left[n_{1}\right]\right]^{\mathrm{T}}, \quad \mathrm{S}\left[n_{1}\right]=\left[S^{1}\left[n_{1}\right], S^{2}\left[n_{1}\right], \ldots, S^{K+P}\left[n_{1}\right]\right]^{\mathrm{T}}$, $\mathbf{N}\left[n_{1}\right]=\left[N_{1}\left[n_{1}\right], N_{2}\left[n_{1}\right], \ldots, N_{M}\left[n_{1}\right]\right]^{\mathrm{T}}$ and $\mathbf{H}\left[n_{1}\right]=\left[\begin{array}{c}H_{1}^{1}\left[n_{1}\right] \cdots H_{1}^{K+P}\left[n_{1}\right] \\ \cdots \\ \cdots \\ H_{M}^{1}\left[n_{1}\right] \cdots H_{M}^{K+P}\left[n_{1}\right]\end{array}\right]$ are the $X$-point FFTs of the corresponding time domain sequences.

\subsubsection{Spatial Distribution of OCIs}

The BS of the desired cell can serve up to $K$ ICUs with $M$ antenna elements. The spatial distribution of users assumes equi-distant ICUs. A maximum of $P$ adjacent interfering cells with one interfering OCI per cell is considered. Due to the use of SDMA and aggressive frequency reuse, a high level of co-channel interference is expected. OCIs' average received powers at a given BS will depend on their propagation paths to their own BSs. ICUs are assigned orthogonal pilots; thus there is no in-cell interference to pilots. Inter-cell pilot interference can be minimized by adopting a frequency reuse partitioning strategy, in which user terminals with low path loss to their BSs, but which are in different cells, have frequency reuse of one, while user terminals experiencing 
higher path loss have a higher frequency reuse factor, and thus experience out-of-cell interference only from more distant cells [Win05].

A representative frequency reuse partitioning scenario is presented in Appendix A in which the frequency reuse factors for terminals within and beyond $70 \%$ of the cell radius are 1 and 3, respectively. It is shown that based on a WINNER wide area propagation model, average received power from each OCI in this deployment scenario is at least 15 $\mathrm{dB}$ below that of ICUs. Other techniques may be employed to reduce out-of-cell interference, such as dynamic channel allocation and scheduling [Win05]. For evaluation of CE performance for SDMA with out-of-cell interference, we assume that one or more of these techniques have been applied, so that uplink OCI signals arrive at a victim BS with an average received power of $-15 \mathrm{~dB},-16.6 \mathrm{~dB}$ or $-18.2 \mathrm{~dB}$ relative to the average power of each in-cell received signal. To simulate a severe out of cell interference, a special case of $6 \mathrm{~dB}$ below the ICU is also considered.

\subsection{Dealing with OCIs}

The performance of the desired ICUs' CE in the presence of OCIs depends largely on the amount of interference offered by the OCIs at a specific time instant. This amount depends on the number and relative power levels of the interfering OCIs. To deal with the inter-cell interference, we may or may not consider higher layer coordination to compensate the OCIs via resource reservations. We categorize the OCIs into two classes, one in which we reserve orthogonal pilot resources (compensated) and the other in which we do not reserve the resources (uncompensated).

\subsubsection{Compensated OCIs}

One of the solutions for handling the inter-cell interference is to keep the training symbols of the OCIs also orthogonal to these of the ICUs. By keeping all the pilots orthogonal to one other, a good initial estimate of the channels of both ICUs and OCIs is achievable. Compensation is done by reserving tones for the OCIs pilots in the ICUs blocks. The idea is depicted in Figure 4.1 and 4.2, with FMT and TMT pilot-multiplexing options respectively. We keep the ICUs and OCIs' training orthogonal by keeping the 
OCIs' pilot locations unused. A further form of compensation is that the desired BS also knows OCI's training.

With these compensations, we obtain two advantages. With orthogonal OCIs' pilots, inter-cell interference does not affect ICUs' channel estimates; moreover, the knowledge of the OCIs channel estimates will further improve the desired ICUs estimation. These two compensations cost more transmission overhead and extra receiver processing. More realistic compensation would be when OCIs' pilots overlap the ICUs pilots, and the BS knows the training sequences sent by the OCIs.

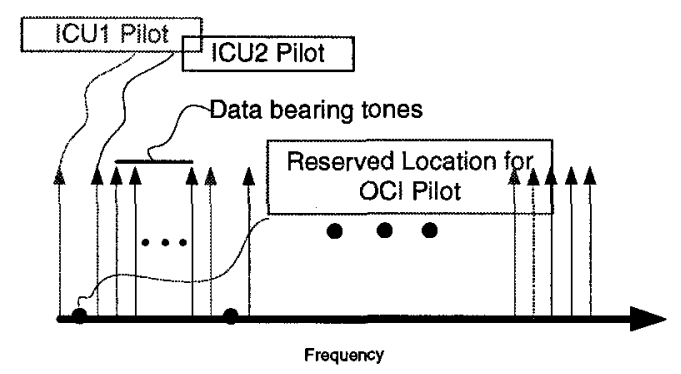

Fig. 4.1. One compensated OCI (FMT example).

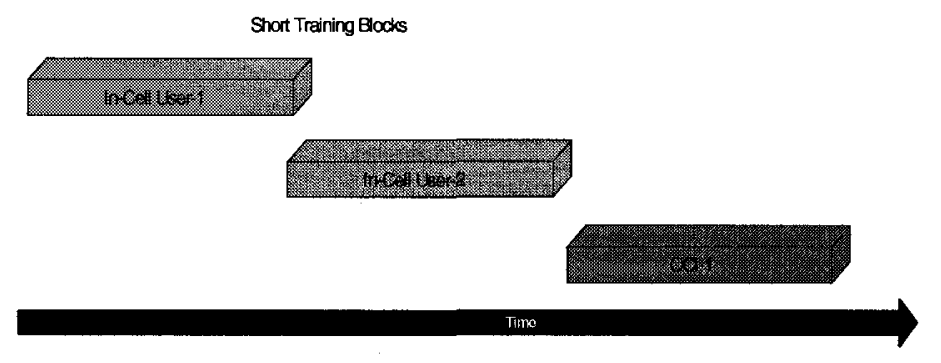

Fig. 4.2. Orthogonal ICUs and an OCI (TMT example).

The above-mentioned forms of compensations can be summarized as:

Option-1: ICUs' training and OCIs' training are kept orthogonal to each other and the desired BS knows training sequences from all users.

Option-2: ICUs' training and OCIs' training are kept orthogonal to each other and the desired BS does not know the OCI's training.

Option-3: ICUs' training and OCIs' training overlap, and the desired BS knows the OCI's training. 


\subsubsection{Uncompensated OCIs}

Allowing for orthogonal out-of-cell pilots requires that each cell leaves unused time or frequency resources for adjacent interfering cells' pilots. By keeping all the pilots orthogonal to each other we may obtain a good initial channel estimates of the ICUs but the compensation will cost resources proportional to the number of ICUs plus OCIs. A more realistic and practical approach in the presence of relatively low power OCIs is not to reserve resources for them. This means that when an OCI appears in a system, its pilots overlap and interfere with the ICU's pilots. This situation is shown in Figure 4.3 and termed uncompensated OCIs in the rest of the thesis. Here we ignore the OCIs during the estimation process. Obviously, with a larger number of such overlapping OCIs, more deteriorated and noisy estimates are anticipated. In analogy to the above three options, the uncompensated case is described as:

Option-4: ICUs' training and OCIs' training overlap, and the desired BS does not know the OCI's training.

In all of these options, all users' pilots are orthogonal to all users' data.

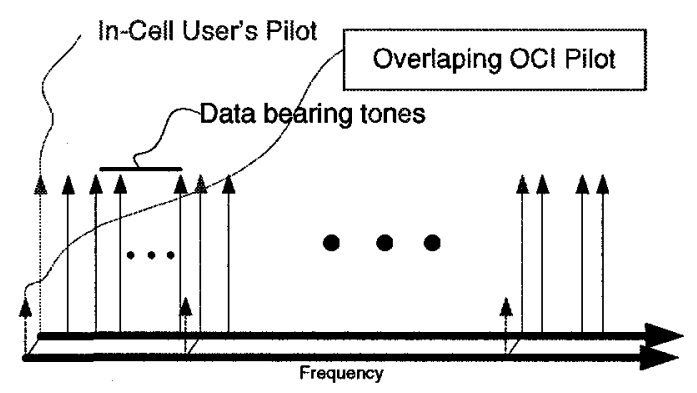

Fig. 4.3. Uncompensated OCIs (FMT example).

\section{Received Array Input during Training:}

In an environment with inter-cell interference, the received vector at the adaptive array during the training phase depends on the type of compensation. Assuming Time Multiplexed Training (TMT), with $K$ ICUs and $P$ OCIs in the system, time averaging over at least $K+P$ short training blocks, one per user, is necessary if all $K+P$ user channels are to be estimated. With non-orthogonal (overlapping) ICUs and overlapping OCIs (whether or not we know the OCIs' training), on the reception of a short training block after FFT we have, 


$$
\breve{Y}_{m}[\bar{n}, \bar{c}]=\sum_{\bar{k}=1}^{K+P} H_{m}^{\bar{k}}[\bar{n}, \bar{c}] \bar{A}^{\bar{k}}[\bar{n}]+N_{m}[\bar{n}, \bar{c}]
$$

where, $m=1,2, \ldots, M, \bar{c}$ represents the index for the pilot-carrying blocks in a frame and $\bar{n}=0,1, \ldots, T_{r}-1 . T_{r}$ is the length of the training block and $\breve{Y}_{m}[\bar{n}, \bar{c}]$ is the received signal at

the $m$ th antenna element, $\bar{A}^{\bar{k}}[\bar{n}]$ is the training sequence of the $\bar{k}$ th user at frequency $\bar{n}$ for $\bar{k}=1,2, \ldots, K+P$, and $N_{m}[\bar{n}, \bar{c}]$ is the AWGN vector. In case of orthogonal ICUs (nonoverlapping) with overlapping OCIs, (4.3) becomes:

$$
\breve{Y}_{m}[\bar{n}, \bar{c}]=\sum_{\bar{k}=1}^{P+1} H_{m}^{\bar{k}}[\bar{n}, \bar{c}] \vec{A}^{\bar{k}}[\bar{n}]+N_{m}[\bar{n}, \bar{c}]
$$

Similarly, in the case of no knowledge of the OCIs at the BS, as far as the receiver processing is concerned, overlapping OCIs' training is treated as an implicit part of $N_{m}[\bar{n}, \bar{c}]$ in (4.4).

\subsection{Simulation Results and Discussions}

To simulate the inter-cell interference along with the intra-cell interference we consider the same signal characteristics and parameters described in Section 2.5.1 for the ICUs and OCIs. Simulation parameters are modified from those used in Chapter 3 and are given in Table 4.1. The number of ICUs in the system could be 1 or 2 , with their pilots orthogonal to each other. The number of training blocks $(N T)$ depends on the total number of users or according to the time-averaging requirements. $\mathrm{C} 2$ urban macro 20path channel model is used [Win01]. A vehicular speed of $50 \mathrm{~km} / \mathrm{hr}$ is used. The power delay profile is given in Table 2.1. Convolutional coding with a 1/2-rate, a constraint length of 7 and the code generator polynomial $[133,171]$ is used. A Viterbi decoder at the receiver is used. Indirect adaptation is used in all simulations throughout the chapter. 
Table 4.1. Modified simulation parameters.

\begin{tabular}{|c|c|}
\hline System Parameters & Values \\
\hline Used sub-carriers $(B)$ & 1024 \\
\hline Modulation Scheme & QPSK \\
\hline Carrier frequency & $3.7 \mathrm{GHz}$ \\
\hline Signal BW & $40 \mathrm{MHz}$ \\
\hline Number of blocks/Code Frame $(F)$ & 12 \\
\hline Number of In-Cell users $(K)$ & $\begin{array}{c}\text { Varies in different } \\
\text { simulation scenarios }\end{array}$ \\
\hline Number of Out of Cell users $(P)$ & $\begin{array}{c}\text { Varies in different } \\
\text { simulation scenarios } \\
\text { Varies in different } \\
\text { simulation scenarios }\end{array}$ \\
\hline Number of receiving elements $(M)$ & Vary from case to case \\
\hline
\end{tabular}

\subsubsection{Comparison between Compensated and Uncompensated OCIs}

In this section we evaluate the average BER performance with different forms of the compensations and placements of OCIs' training sequences. A QPSK modulation based SC-FDE system is simulated for a range of received SNRs, each with 2,000 random channel realizations. Results after LE are shown with 256 pilots used in the first and twelfth data block in each 12-block code frame. The number of receiving antenna elements is $3(M=3)$. The transmitted signal arrives at each receiving antenna through an independent $\mathrm{C} 2$ channel with a vehicle speed of $50 \mathrm{~km} / \mathrm{hr}$. We assume that the data of all the users $(K+P)$ overlaps over the entire time-frequency frame. In Figure 4.4, we consider two ICUs $(K=2)$ and one OCI $(P=1)$ with the simulation scenarios given in Table 4.2.

Table 4.2. Simulation scenarios.

\begin{tabular}{|l|l|}
\hline $\begin{array}{l}\text { TMT-ortho,UC, } \\
M=3, K=2, P=1\end{array}$ & $\begin{array}{c}\text { ICUs pilots are orthogonal and ICUs' and OCIs' training overlap } \\
\text { while the BS does not know the OCI's training and does not estimate } \\
\text { (uncompensated) }\end{array}$ \\
\hline $\begin{array}{l}\text { TMT-ortho,C- } \\
\text { ONK }\end{array}$ & $\begin{array}{l}\text { ICUs' and OCIs' training are kept orthogonal to each other while the } \\
M=3, K=2, P=1\end{array}$ \\
\hline TMT-ortho,C- & ICUs' training and OCIs' training are kept orthogonal to each other \\
\hline
\end{tabular}




\begin{tabular}{|l|c|}
\hline KT & while the BS knows OCI's training and estimates OCI channel. \\
$M=3, K=2, P=1$ & \\
\hline TMT-ortho, & \\
$M=3, K=2, P=0$ & \\
No OCIs & ICUs' training orthogonal to each other. \\
\hline PCSI & PCSI with no OCIs. \\
$M=3, K=2, P=0$ & \\
No OCI & \\
\hline
\end{tabular}

No Inter-cell interference and PCSI curves are also shown for comparison purposes. 'KT' in the legend in the Figure 4.4 stands for the 'Known Training' of the OCIs. Similarly, 'TNK' represents the 'Training of the OCIs Not Known'. The average received relative power of the OCI is 1/16th of that of the ICU's power. The TMT pilot multiplexing technique is used. Performance gain that is achieved with compensation in terms of additional knowledge about the OCIs' training is evident. A gain of approximately $1 \mathrm{~dB}$ is obtained by reserving resources for the OCIs.

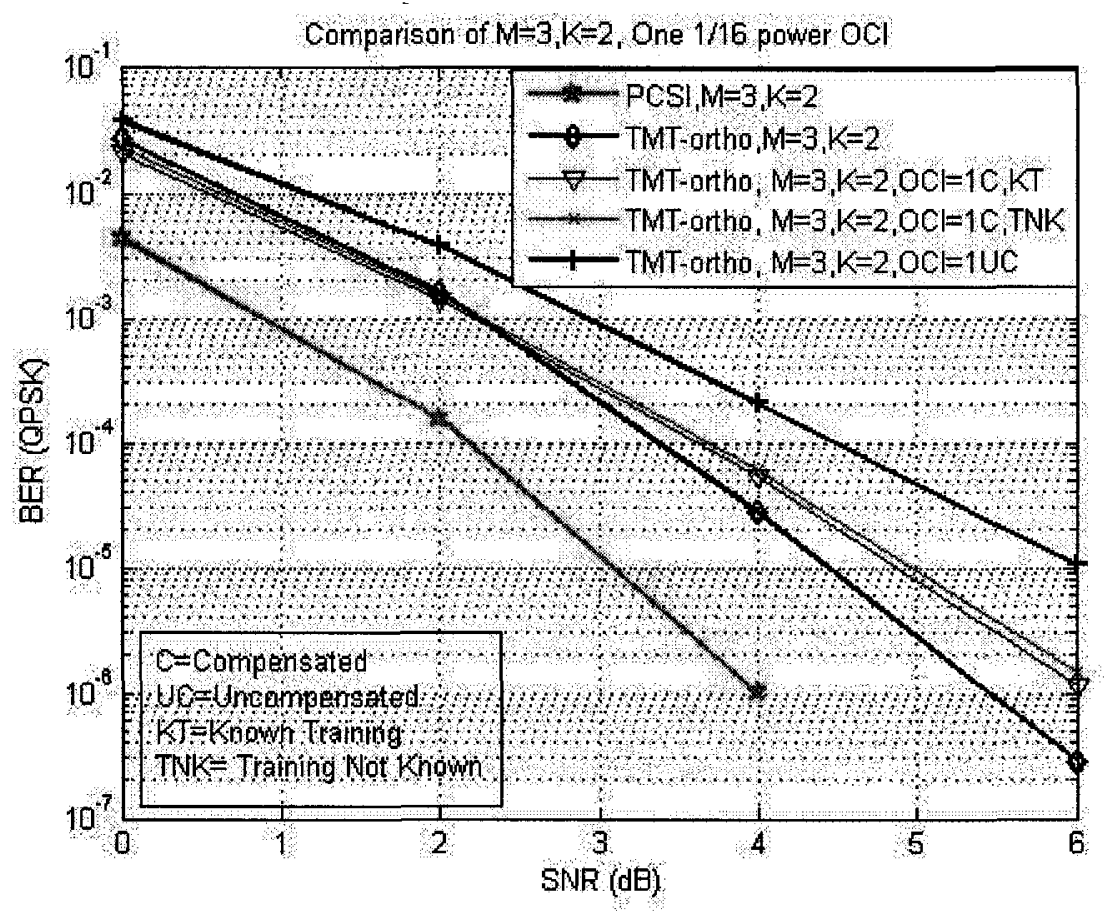

Fig. 4.4. Average BER-Uncompensated and compensated OCI, OCI training known or unknown (relative power=1/16), $M=3, K=2$. 
As the OCI in Figure 4.4 is quite weak, equalizer coefficients computed with known OCI channel do not help resulting in having not much performance difference in knowing or not knowing the training of the OCI. This difference becomes significant if the interfering OCI becomes stronger. This is verified with a Quarter Power (QP) OCI (around $6 \mathrm{~dB}$ below the ICUs) shown in Figure 4.5, which depicts that knowing or not knowing the powerful OCIs' training play an important role (around 1.8dB SNR degradation by not knowing the OCI's training) in CE process of the desired ICU. QP is a severe assumption and is only made to simulate a worst-case scenario.

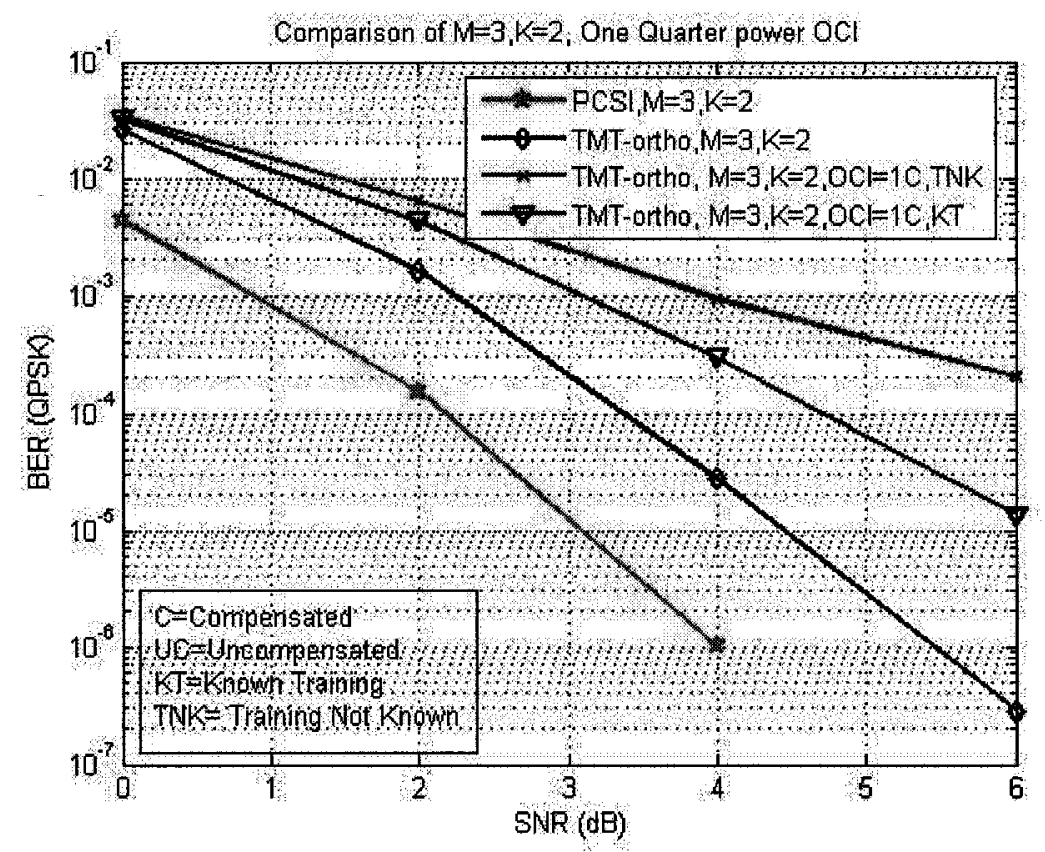

Fig. 4.5. Average BER-Compensated OCI, OCI training known or unknown (relative power=1/4) $M=3, K=2$.

\subsubsection{Uncompensated OCIs with no Intra-cell Interference}

With an increasing number of OCIs, compensation via reserving resources to make their training orthogonal becomes expensive in terms of transmission overhead. Therefore, we focus on the case when all the OCIs' training overlaps (either known to the BS or not). In this section we consider no intra-cell interference. We consider two equi-power overlapping OCIs $(P=2)$ and no intra-cell interference $(K=1)$ with TMT training sequence arrangement shown in Figure 4.6. 


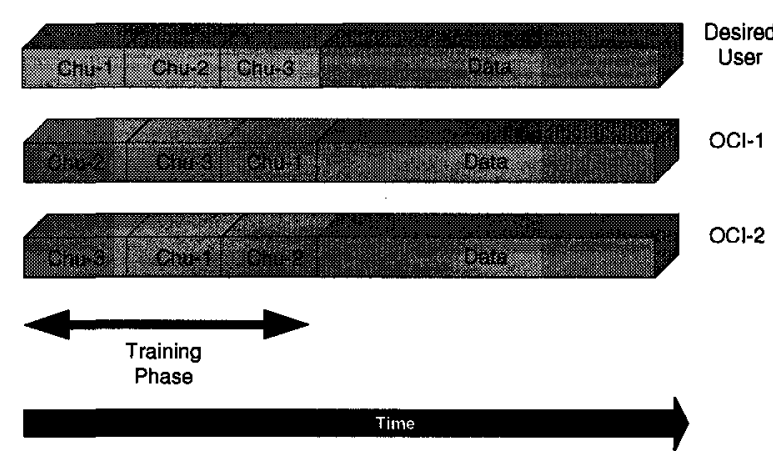

Fig. 4.6. Overlapping OCIs $(K=1, P=2)$.

The effect of knowing the OCIs' training and explicitly estimating their channels, on the average BER with different average received SNRs per antenna element is shown in Figure 4.7. Each of the 2 OCIs has relative power $6 \mathrm{~dB}$ below the ICU (around quarter of the ICU power). $\mathrm{C} 2$ channel with a vehicle speed of $50 \mathrm{~km} / \mathrm{hr}$ is considered. A larger gain at higher SNRs due to the known training of the OCIs is obvious from the figure.

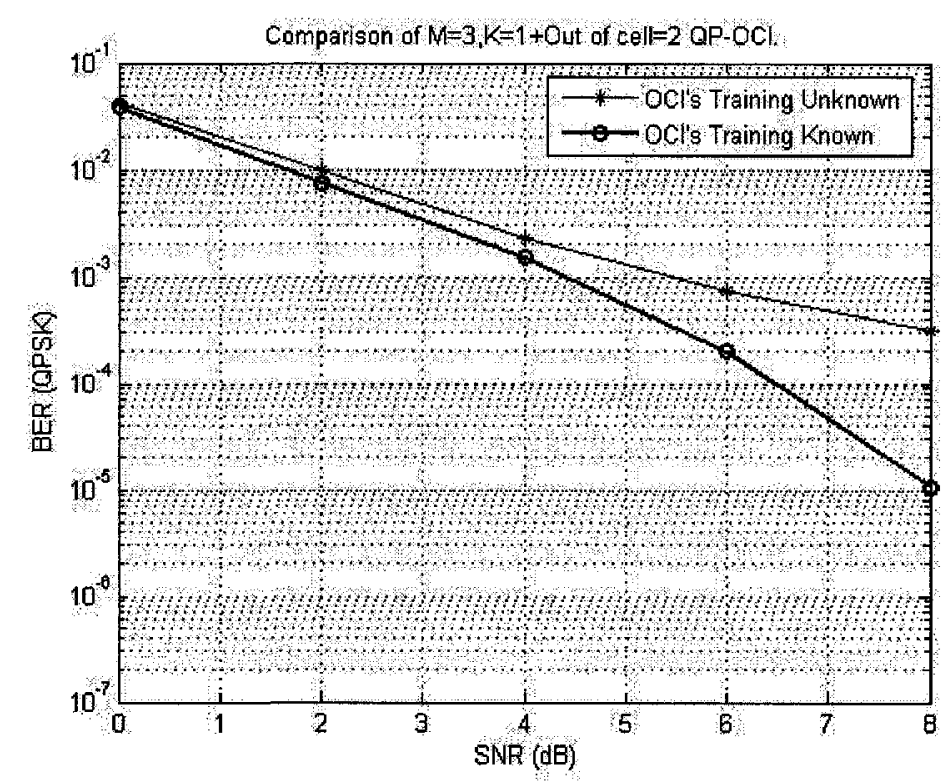

Fig. 4.7. Average BER Comparison between known and unknown training of overlapping OCIs, $M=3, K=1, P=2$ (relative power=1/4).

In Figure 4.8 an increase in the number of overlapping OCIs from 1 to 6 is simulated. With one ICU and up to 6 overlapping OCIs, coordinated Chu sequence-based $K+P$ short 
training blocks (TMT) are used. Average BER performance with different number of equal average power OCIs with 3, 4 and 5 antenna elements at the receiver is illustrated. The average received power from each of these OCIs is $6 \mathrm{~dB}$ below the ICU. There is no intra-cell interference in the system. Average BER reduction with the employment of 3,4 and 5 antenna elements at the BS is obvious. Without knowing the OCIs' training and at an average BER of $10^{-3}, 3$ antenna elements can support upto 2 QP-OCIs, 4 antenna elements can support around 3.5 OCIs and 5 antenna elements can support up to 5 OCIs. Knowing the training sequences of the OCIs at the desired BS enables us to support more OCIs at a certain level of BER. But this achievement costs additional receiver processing and extra pilot overhead for each of the OCI. Simulations are carried out at the average received SNR of $6 \mathrm{~dB}$ at each receiving antenna element. Results are obtained after performing LE. C2 channel with a vehicle speed of $50 \mathrm{~km} / \mathrm{hr}$ is considered.

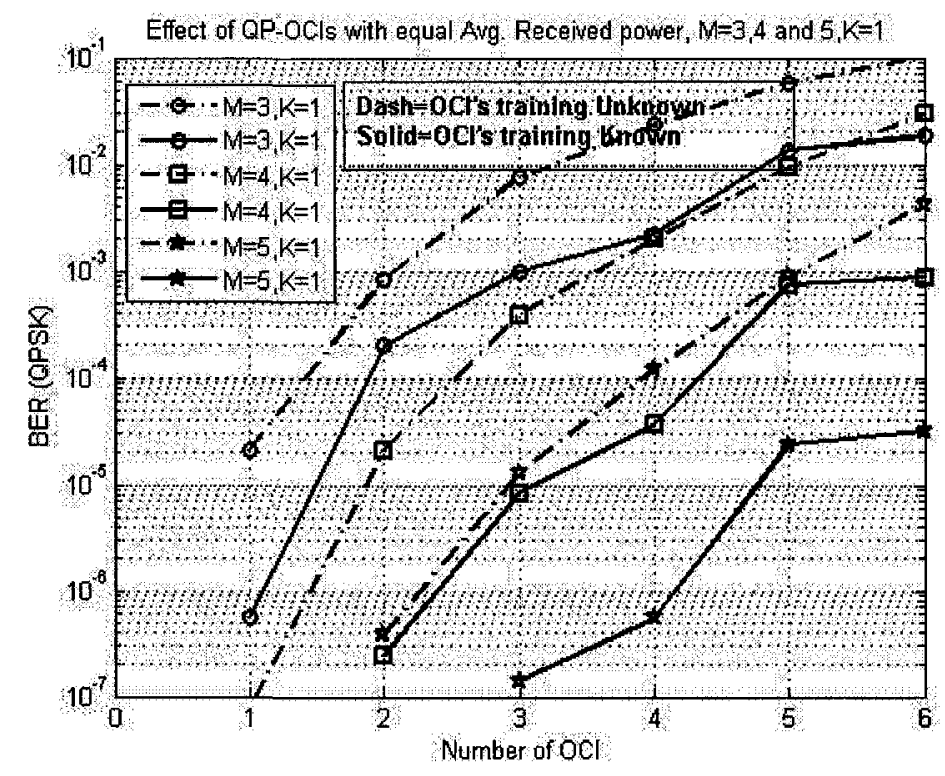

Fig. 4.8. Average BER- Performance with diversity and known and unknown training,

$$
P=1,2, \ldots, 6, M=3 \text { and } 4 \text { and } 5, K=1 \text {. }
$$

In most of the practical scenarios, OCIs would not be sitting on a ring around the desired BS. The number and power of the OCIs will vary with time. Figure 4.9 shows the effect on the CE performance of the desired ICU, with different average power levels of the OCIs due to the distance attenuation. 4 OCIs with no intra-cell interference and 3 BS 
antenna elements are assumed. It should be noted that the advantage of estimating the OCIs' channels with their known training sequences is less significant at longer distances. Simulations are carried out at an average received SNR of $6 \mathrm{~dB}$ at each receiving antenna element. LE results are illustrated with a path loss exponent of $\Omega=4$.

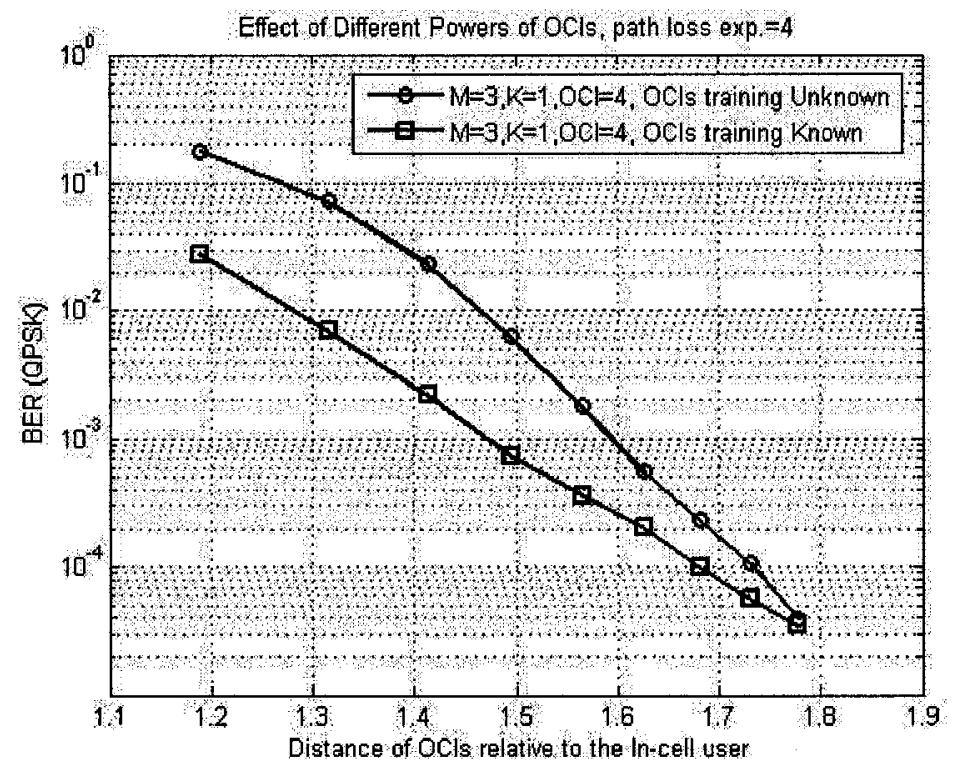

Fig. 4.9. Average BER-Effect of distance attenuation of OCIs, $P=4, M=3, K=1$.

Results in Figure 4.9 show that at larger distances of OCIs (weak OCIs), the advantage of knowing their training sequences is diminished. Therefore, treating them uncompensated becomes a more practical assumption. Therefore, we will be using uncompensated scenario in later sections of this chapter and also in the remainder of the thesis.

One advantage of having weak OCIs is time averaging over fewer short training blocks (NT per ICU), than the number of available users $(K+P)$. Results are shown in Figure 4.10. As described in Section 4.2.1, we simulate OCIs' relative powers of $-15 \mathrm{~dB}$, $16.6 \mathrm{~dB}$ and $-18.2 \mathrm{~dB}$ relative to the ICU [See Appendix A]. Simulations are carried out at an average received SNR of $6 \mathrm{~dB}$ at each receiving antenna element. $\mathrm{LE}$ and $\mathrm{C} 2$ channel with a vehicle speed of $50 \mathrm{~km} / \mathrm{hr}$ is considered. Two different pilot overheads are compared. One has short training blocks equal to the total number of users $(N T=K+P)$, and the other has 2 (fixed) short training blocks (NT=2). Average BER corresponding to a maximum of 6 OCIs is shown. There are two antenna elements at the BS. We observe degradation in the performance due to less time averaging over the short training blocks. 
But averaging over two training blocks still gives an acceptable range (BER $<10^{-4}$ with 4 OCIs @-15dB) and a good trade off with the reduction in the pilot overhead.

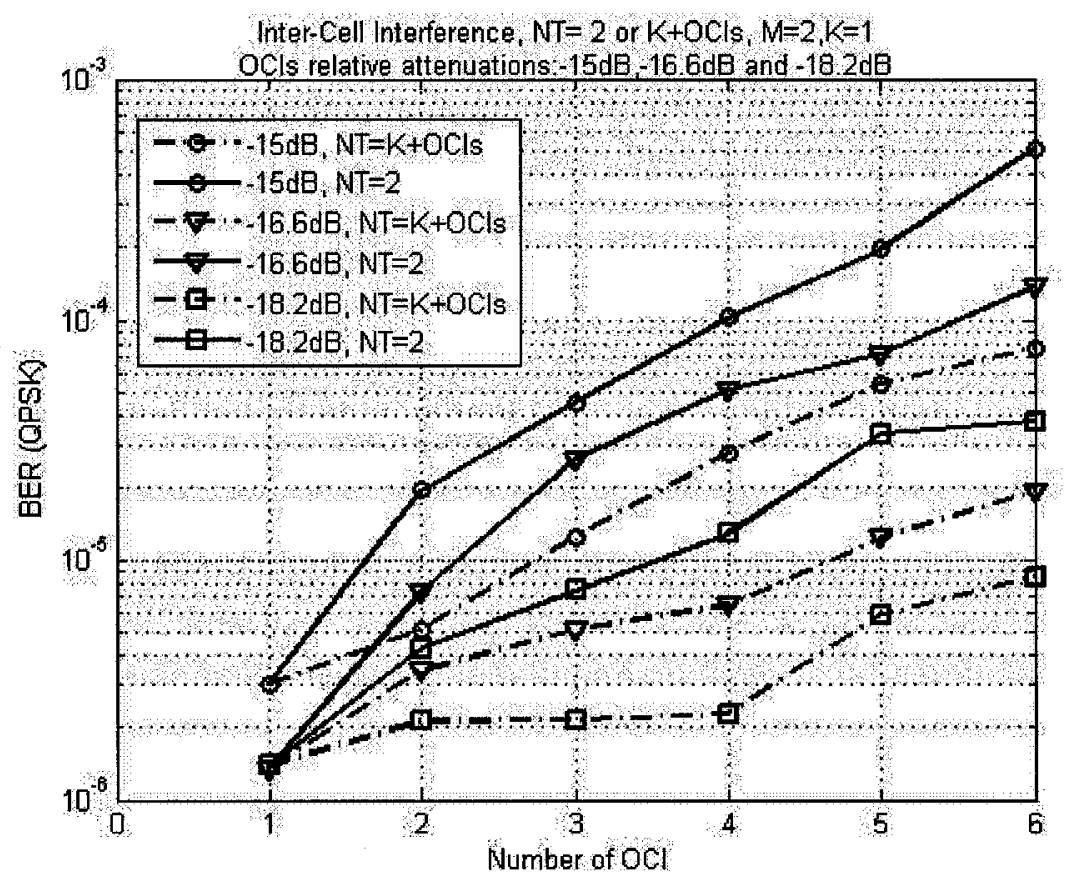

Fig. 4.10. Average BER-Different power levels of OCIs (Uncompensated OCIs- no intracell interference), $P=1,2, \ldots, 6, M=2, K=1, N T=2$ and $(K+P)$.

\subsubsection{Uncompensated OCIs with Intra-cell Interference}

In this section we illustrate the effects of the inter-cell interference in the presence of intra-cell interference. Figure 4.11 shows different possible scenarios of the appearance of the short training blocks of the in-cell and out of cell users. With 2 ICUs and 6 OCIs, we consider 3 different simulation scenarios labeled as 'case 0 ', 'case 1' and 'case 2 '.

- 'Case 0': All users' $(K+P)$ training overlap (ICUs are non-orthogonal).

- 'Case 1': ICUs training is kept orthogonal but all the OCIs' training overlaps one of the ICU.

- 'Case 2': is a load-sharing situation in which one half of the OCIs' training overlaps one of the ICU and the other half of the OCIs overlaps the other ICU. 


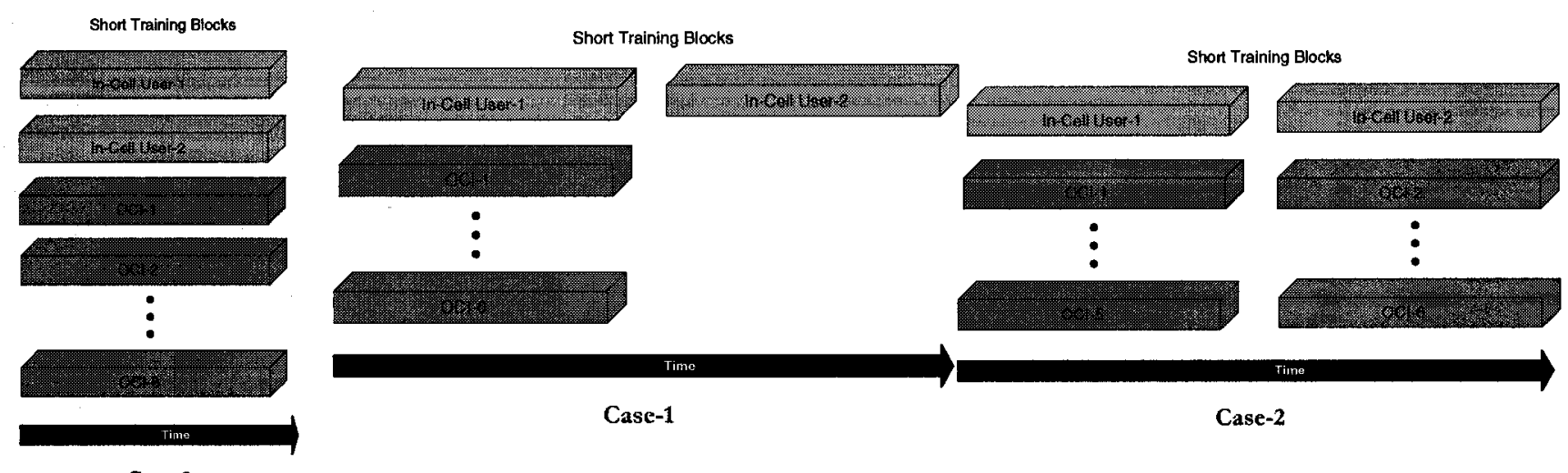

Case-0

Fig. 4.11. Different cases of appearance of ICUs' and OCIs' training blocks (TMT).

In Figure 4.12, average BER performance for the three above-mentioned scenarios are compared against the case in which there is only one ICU in the system (no inter or intracell interference). The relative powers of the interfering OCIs are $6 \mathrm{~dB}$ below the ICUs. Simulations are carried out with 4 antenna elements at the BS. The number of short training blocks $(N T)$ is equal to the total number of users $(K+P)$. It is evident that the load-sharing scenario ('case 2') offers the best performance in terms of average BER, considering the same amount of overhead. Simulations are carried out with LE and at a level of $6 \mathrm{~dB}$ of SNR at each antenna element.

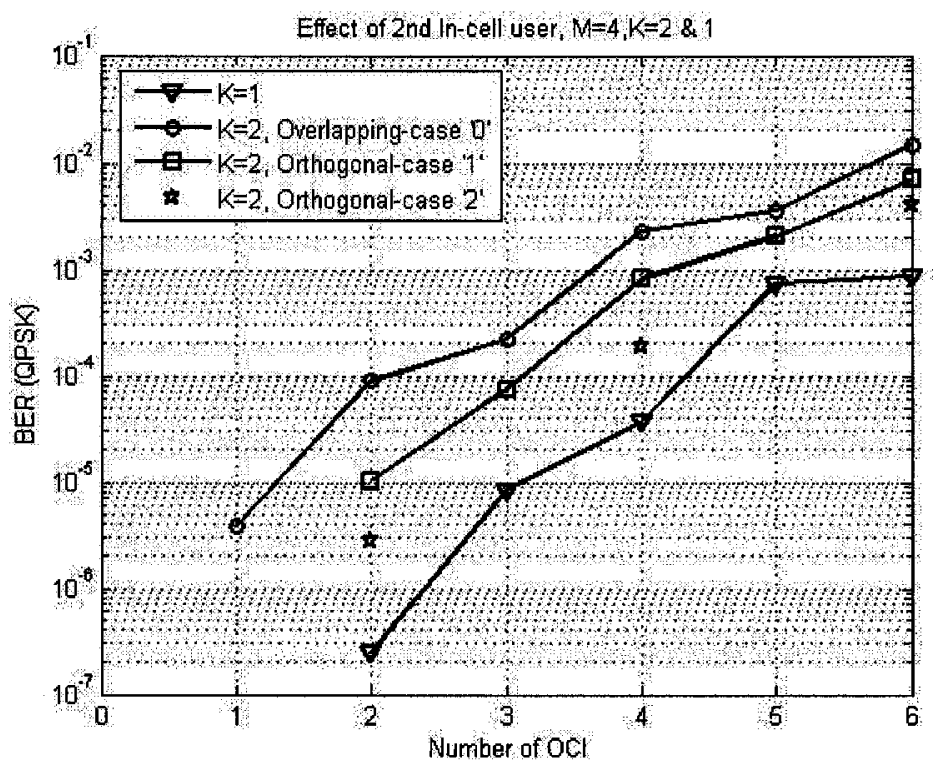

Fig. 4.12. Average BER- Different cases of appearance of OCIs (Uncompensated OCIs with intra-cell interference). 


\subsection{Modeling of OCIs with a Gaussian Approximation (GA)}

In the section we analyze the $\mathrm{CE}$ error and theoretically evaluate how the $\mathrm{CE}$ error affects the performance of the average MSE at the equalizer output in a SC-FDE system in the presence of OCIs. The out of cell interference is modeled by using a Gaussian Approximation (GA) approach. In GA, we assume the entire out of cell interference as white Gaussian noise. To apply GA, the noise variance applied in the MMSE criteria has to be scaled appropriately. The approximated received signal is given by:

$$
\mathbf{Y}[n]=\mathbf{S}[n] \mathbf{H}[n]+\mathbf{N}[n]+\mathbf{N}_{\text {OCI }}[n]
$$

where $\mathbf{S}[n]$ is the In-cell transmitted data, $\mathbf{Y}[n]$ is the $M \times 1$ received array input, $\mathbf{H}[n]$ is the channel matrix, $\mathbf{N}[n]$ represents the AWGN and $\mathbf{N}_{O C I}[n]$ represents the interference caused by the unknown OCIs. Equalization according to the MMSE criteria minimizes the mean square value of the error between the transmitted signal and the output of the equalizer. The error signal is given by:

$$
\partial[n]=\mathbf{S}[n]-\mathbf{W}[n] \mathbf{Y}[n]
$$

where $\mathbf{W}[n]$ is $M \times 1$ linear array weight vector. The MMSE is given by:

$$
J_{\min }=E\left(|\partial[n]|^{2}\right)
$$

And can be minimized by applying the orthogonality principle [Hay02].

$$
\begin{aligned}
E\left[\partial \mathbf{Y}^{*}\right] & =0 \\
& =E\left[(\mathbf{S}-\mathbf{W Y})\left(\mathbf{H S}+\sigma_{n}^{2}+\sigma_{\mathrm{OCI}}^{2}\right)\right]
\end{aligned}
$$

The MMSE is minimal if the equalizer coefficient $\mathbf{W}$ is selected such that the error $\partial$ is orthogonal to the received array input signal $\mathbf{Y}$. That is, the modified MMSE coefficients are

$$
\mathbf{W}[n]=\left[\mathrm{H}[n] \mathrm{H}[n]^{\mathrm{H}}+\left({\sigma_{n}}^{2}+\sigma_{\mathrm{OCI}}^{2}\right) \mathbf{I}_{M}\right]^{-1} \mathrm{H}[n]
$$

The inter-cell interference noise term is added at the receiver side to the received desired signal including the thermal noise term. The variance of the inter-cell interference noise term can be expressed by

$$
\sigma_{\mathrm{OCI}}^{2}=\sum_{p=1}^{P} E_{O C I}^{p} \varepsilon_{O C I}^{p}
$$


where $P$ is the total number of interfering OCIs, $\varepsilon_{\mathrm{OCI}}$ represents the attenuation factor of each ( $p$ th) interfering OCI due to the applied propagation model for the transmitted signals, $E_{O C I}$ is the transmitted OCI symbol energy.

Figure 4.13 and 4.14 illustrate the effectiveness of the GA approach. It shows that low power (@-18.2dB) and large in number ( $P=5$ and 6) OCIs can be approximated by a GA approach.

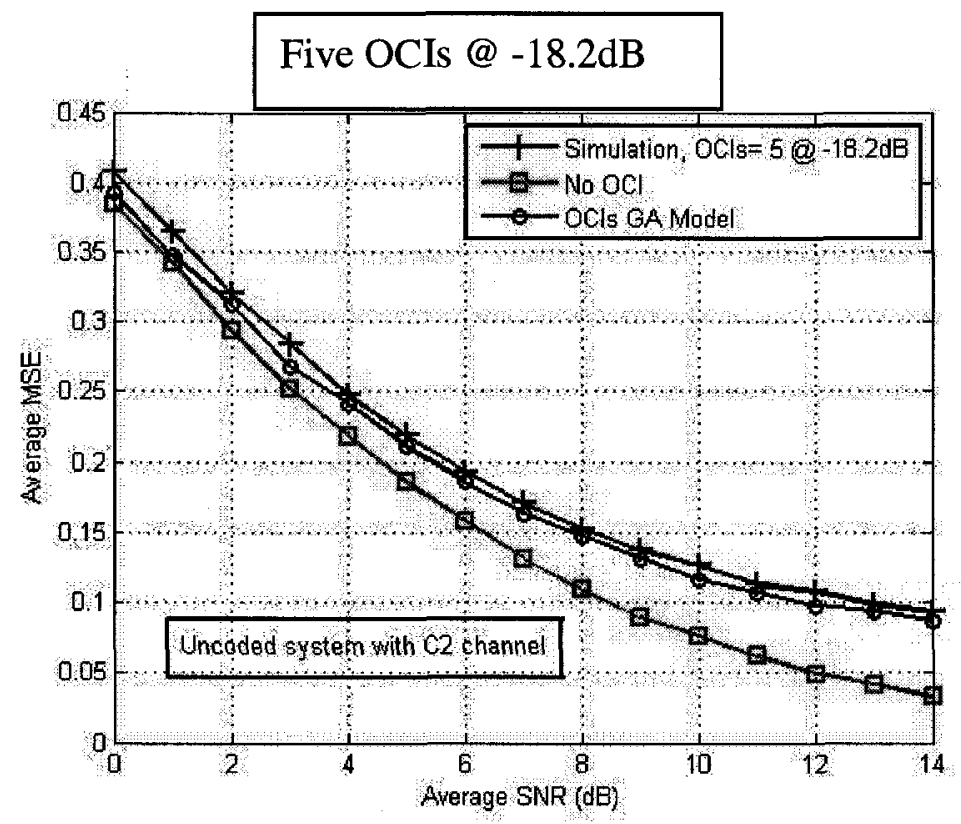

Fig. 4.13. Average MSE at the LE output with PCSI, OCI modeling with GA ( $P=5$ @ $18.2 \mathrm{~dB})$. 


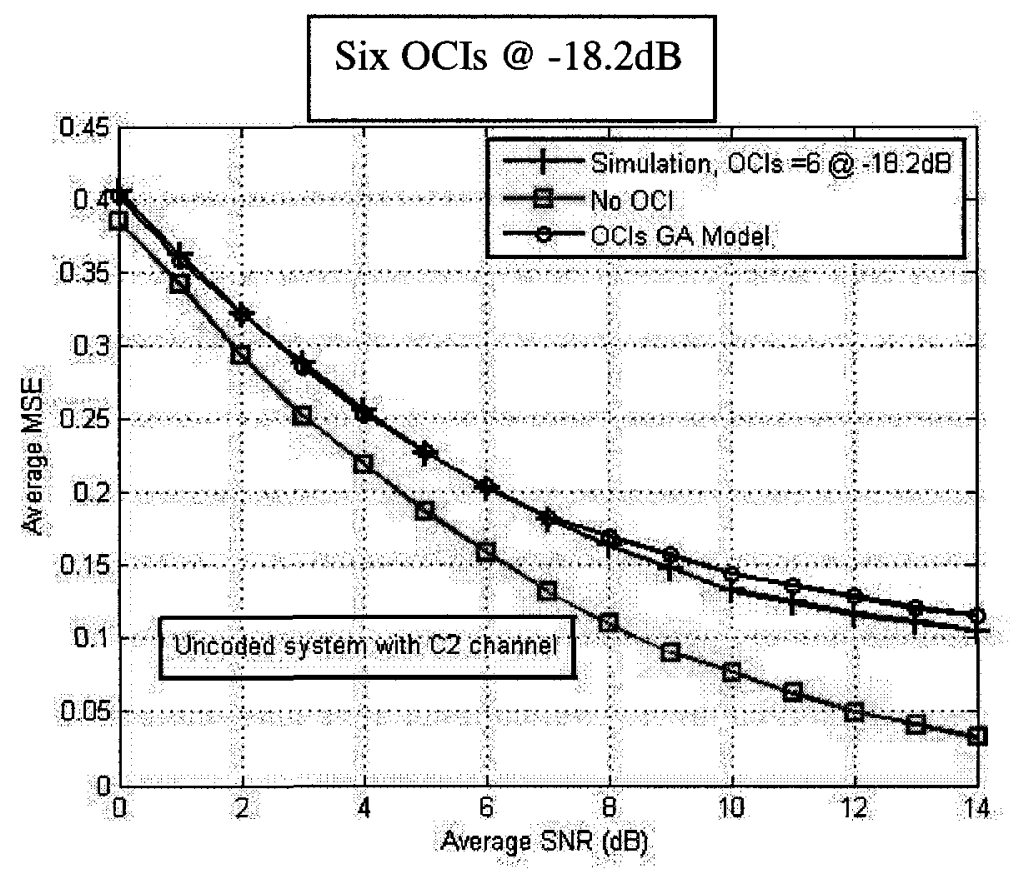

Fig. 4.14. Average MSE at the LE output with PCSI, OCI modeling with GA ( $P=6 @$ 18.2dB).

\subsection{Summary}

In this chapter, the CE aspects in the presence of inter-cell interference with or without intra-cell interference were explored. To deal with inter-cell interference orthogonal outof-cell pilots requires that each cell leaves unused time or frequency resources for adjacent interfering cells' pilots. This compensation consumes resources and may be wasteful as well, as most of the weak OCIs would not appear very often. In this situation the overlapping OCIs' pilots become more significant and practical. It is concluded that the inter-cell interference could be handled with orthogonal in-cell pilots and OCIs could be dealt with by a combination of the spatial diversity and additional time averaging over several short training blocks. 


\section{Chapter 5}

\section{MIMO Soft Decision-based Iterative Block DFE}

\subsection{Introduction}

In this chapter we propose a low complexity multi-user or MIMO Soft Decision-based Iterative, Block-based channel FDE (SD-IBDFE) algorithm. The proposed iterative equalizer structure is a low complexity non-linear soft decision feedback technique that not only mitigates the ISI but is also designed to suppress the co-channel interference without incurring significant error propagation effects. Estimated CFRs are fed into the SD-IBDFE and within the SD-IBDFE iterations the channel estimates remain the same. We will be using the SD-IBDFE or its modified versions throughout in this thesis in addition to other proposed algorithms. This chapter deals with the description and mathematical details of the SD-IBDFE and presents evaluation of the algorithm via simulations with or without co-channel OCIs.

\subsection{Multi-User Soft Decision-based Iterative Equalization}

Decision Feedback Equalization (DFE) is a non-linear treatment used to cancel the ISI induced by the past symbols without any noise enhancements. [BT02b] shows the pioneer work on DFE in single carrier systems with frequency domain feed forward equalizer. [FAB02] and [WKW04] also address a similar DFE with frequency domain feed forward and time domain feedback equalization. DFE in single carrier with single link is extended to MIMO systems in [TPD03] with frequency domain feed forward filter and time domain feedback processing. Higher complexity is one of the major concerns due to time domain non-linear processing. [TPD03] is an extension to the work presented 
in [BT02b] and [FAB02] with MIMO channels. In the following section we present an overview of the block-based decision feedback equalization schemes.

\subsubsection{Overview of the SD-IBDFE}

A hard decision based Iterative Block DFE (IBDFE), with block-based frequency domain feedback processing in contrast to the symbol-based conventional time domain DFE, is introduced in [BT02a]. Such structures reduce the complexity of the non-linear processing compared to the time domain approaches. This complexity reduction becomes significant in channels having long delay spreads. In IBDFE, since the feedback loop takes into account not just the decisions for each block but also the overall block reliability, we have small error propagation. This technique is later extended to diversity scenarios [DGE04] and layered space-time schemes [DKF04]. Smaller error propagation and additional diversity gain are the main motivation for considering the IBDFE structure.

[TB04] proposed a Soft Decision-based Iterative Block Decision Feedback Equalizer (SD-IBDFE) in a Single-Input Single-Output (SISO) channel environment. In this section, we propose and evaluate a modified multi-user or Multiple Input Multiple Output (MIMO) SD-IBDFE. The SD-IBDFE presented here is a modified version of that proposed in [TB04] that uses an approach similar to [DGE04], with the difference that soft decisions are used instead of hard decisions. In this SD-IBDFE, a conditional MSE difference between the symbols and their soft decisions in each iteration is computed by using the corresponding equalizer's output. The objective is to minimize the conditional MSE with respect to the feed forward and feedback coefficients. Both feed forward and feedback filters are implemented with efficient FFTs resulting in lower complexity compared to the SC-FDE with DFE described in [BT02a], [TPD03] and [WKW04], in which the feed back operations are implemented in time domain. The proposed SDIBDFE helps to mitigate ISI and also suppresses co-channel interference. 


\subsubsection{SD-IBDFE Coefficients Computation}

In this section we describe some mathematical details of the computation of the feed forward and feedback filter coefficients for the SD-IBDFE. The adaptation of the feed forward and feedback equalizer coefficients and the resulting MSE are computed. A simplified block diagram is shown in Figure 5.1.

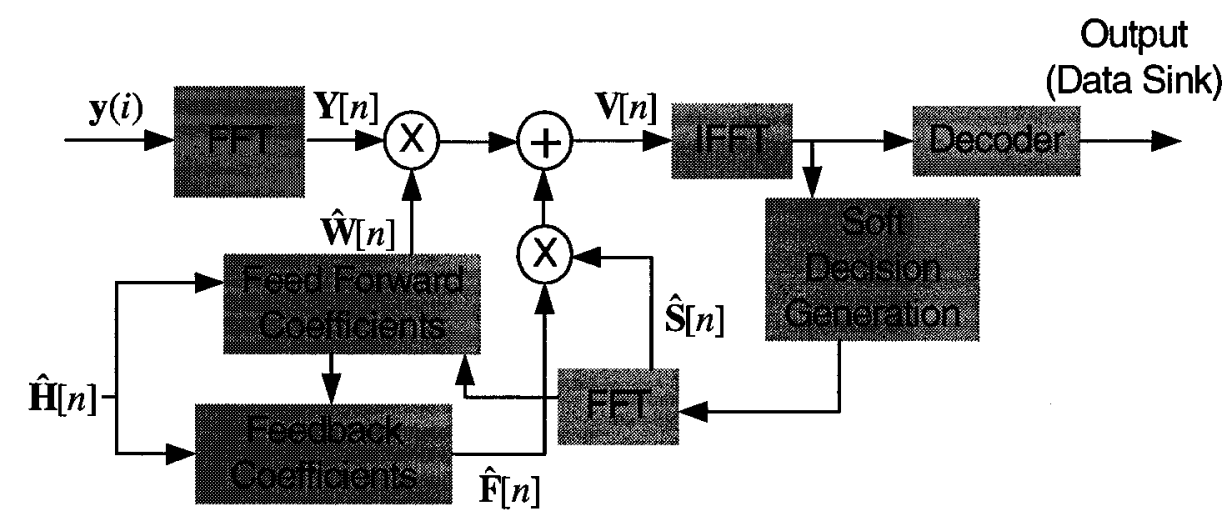

Fig. 5.1. Block diagram of SD-IBDFE.

The SD-IBDFE considered contains two separate frequency domain filters, namely the forward $(\hat{\mathbf{W}}[n])$ and feedback filters ( $\hat{\mathbf{F}}[n])$, which are jointly optimized in each iteration to minimize the error propagation. In order to determine the filter coefficients the following assumptions are made:

- The noise samples have zero mean and variance $\sigma_{n}^{2}: E\left[\mathbf{N}[n] \mathbf{N}[n]^{*}\right]=B \sigma_{n}^{2} \mathbf{I}_{M}$ where $\mathbf{I}_{M}$ is the identity matrix of size $M$ and $B$ is the block size.

- Data symbols and noise samples are statistically independent.

- Data symbols have zero mean and unit variance: $E\left[\mathbf{S}[n] \mathbf{S}[n]^{*}\right]=B \mathbf{I}_{K}$

The frequency domain antenna array input vector is given by:

$$
\mathbf{Y}[n]=\mathbf{H}[n] \mathbf{S}[n]+\mathbf{N}[n], \quad n=0,1, \ldots, B-1
$$

where

$$
\mathbf{Y}[n] \in \mathrm{C}^{M \times 1}, \mathbf{H}[n] \in \mathrm{C}^{M \times K}, \mathbf{S}[n] \in \mathrm{C}^{K \times 1}, \mathbf{N}[n] \in \mathrm{C}^{M \times 1} .
$$

Frequency domain forward equalizer coefficients (zeroth iteration, $i=0, \mathrm{LE}$ ) based on estimated CFR are given by: 


$$
\hat{\mathbf{W}}^{i=0}[n]=\left[\hat{\mathbf{H}}[n] \hat{\mathbf{H}}[n]^{\mathrm{H}}+\sigma_{n}^{2} \mathbf{I}_{M}\right]^{-1} \hat{\mathbf{H}}[n]
$$

where $\hat{\mathbf{H}}[n] \in \mathrm{C}^{M \times K}, \hat{\mathbf{W}}[n] \in \mathbb{C}^{M \times K}$.

The equalizer output at iteration zero $(i=0)$ is given by:

$$
\mathbf{V}^{i=0}[n]=\hat{\mathbf{W}}^{i=0}[n]^{\mathrm{H}} \mathbf{Y}[n], \quad \mathbf{V}[n] \in \mathrm{C}^{K x l}
$$

Equalizer output samples are the $B$-point IFFT of the equalizer output:

$$
\mathbf{v}^{i=0}(q)=\mathbf{F}_{B}^{-1}\left(\mathbf{V}^{i=0}[n]\right), \mathbf{v}(q) \in C^{K x l}
$$

where $q=0,1, \ldots, B-1$ and $\mathrm{F}_{B}^{-1}$ represents B-point IFFT. The time domain equalizer output matrix $\mathbf{v}^{i} \in C^{K x B}$ at iteration $i$ can be expressed by:

$$
\mathbf{v}^{i}=\left[\mathbf{v}_{1}^{i}, \mathbf{v}_{2}^{i}, \ldots, \mathbf{v}_{K}^{i}\right]^{\mathrm{T}}
$$

where $\mathbf{v}_{k}^{i}=\left\lfloor v_{k}^{i}(0) v_{k}^{i}(1) \ldots v_{k}^{i}(B-1)\right], k=1,2, \ldots, K$.

$v_{k}^{i}(q)$ is assumed to be a complex Gaussian random variable with mean $s_{k}(q)$ and variance $\vec{J}^{i-1}$.

The corresponding MSE for the equalizer at $i=0$ is given by:

$$
\vec{J}^{i=0}=\frac{1}{B^{*} K} \sum_{n=0}^{B-1} \operatorname{tr}\left[\left(\hat{\mathbf{W}}^{i=0}[n]^{\mathrm{H}} \hat{\mathbf{H}}[n]-\mathbf{I}_{K}\right)\left(\hat{\mathbf{W}}^{i=0}[n]^{\mathrm{H}} \hat{\mathbf{H}}[n]-\mathbf{I}_{K}\right)^{\mathrm{H}}+\sigma_{n}^{2} \hat{\mathbf{W}}^{i=0}[n] \hat{\mathbf{W}}^{i=0}[n]^{\mathrm{H}}\right]
$$

where $t r$ represents trace of a matrix.

The soft feedback symbols for QPSK equi-probable symbols are formed by finding the conditional expectation of the data symbols, given equalizer outputs from the previous iteration $i-1$.

$$
\hat{\mathbf{s}}^{i}(q)=E\left(\mathbf{s}(q) \mid \mathbf{v}^{i-1}(q)\right) \quad i=1,2, \ldots
$$

For the $k$ th user and $i-1$ iteration, the conditional expectation is computed as:

$$
\hat{s}_{k}^{i-1}(q)=E\left(s_{k}(q) \mid v_{k}^{i-1}(q)\right)=\frac{\sum_{\text {constellation elements }} \vec{p}\left(v^{i-1}(q) \mid \vec{\gamma}\right) p(\vec{\gamma})}{\sum_{\text {constellation elements } \vec{\gamma}} p\left(v_{i}^{i-1}(q) \mid \vec{\gamma}\right) p(\vec{\gamma})} \quad i=1,2, \ldots
$$

The soft decision $\hat{s}_{k}^{i-1}(q)$ at the $i$ th iteration depends only on the corresponding equalizer output $v_{k}^{i-1}(q)$ for a given data symbol $s_{k}(q)$. The conditional density function is given by: 


$$
p\left(v_{k}^{i-1}(q) \mid \vec{\gamma}\right)=\frac{1}{2 \pi \vec{J}^{i-1}} \exp \left(-\frac{\left|\vec{\gamma}-v_{k}^{i-1}(q)\right|^{2}}{\vec{J}^{i-1}}\right) \quad i=1,2, \ldots
$$

For QPSK modulation, $\vec{\gamma}=\frac{1}{\sqrt{2}}( \pm 1 \pm j)$.

The soft decisions are computed as:

$$
\hat{\mathbf{s}}^{i-1}(q)=\frac{1}{\sqrt{2}}\left[\tanh \left(\frac{\sqrt{2} \operatorname{Re}\left(\mathbf{v}^{i-1}(q)\right)}{\bar{J}^{i-1}}\right)+j \tanh \left(\frac{\sqrt{2} \operatorname{Im}\left(\mathbf{v}^{i-1}(q)\right)}{\bar{J}^{i-1}}\right)\right]
$$

where $\operatorname{Re}(x)$ and $\operatorname{Im}(x)$ represents the real and imaginary part of a complex number $x$ respectively and $\tanh (x)$ represents the tangent hyperbolic function. Here we define the conditional mean squared difference between the data symbols and their soft decision at $i$ th iteration as:

$$
\Omega_{k}^{i}(q)=E\left(\left|s_{k}(q)\right|^{2} \mid \mathbf{v}^{i-1}(q)\right)-\left|\hat{s}_{k}^{i-1}(q)\right|^{2}
$$

Here we define a diagonal matrix $\overrightarrow{\boldsymbol{\Omega}}^{i}$ given by

$$
\overrightarrow{\boldsymbol{\Omega}}^{i}=\left[\begin{array}{lcc}
\Omega_{1}^{i}(q) & & \mathbf{0} \\
0 & \Omega_{2}^{i}(q) & \mathbf{0} \\
\vdots & \ddots & \vdots \\
\mathbf{0} & \multicolumn{3}{r}{\Omega_{K}^{i}(q)}
\end{array}\right]
$$

Let $\boldsymbol{\Omega}_{v}^{i}$ be a row vector containing the diagonal elements of $\overrightarrow{\boldsymbol{\Omega}}^{i}: \boldsymbol{\Omega}_{v}^{i}=\left[\Omega_{1}^{i}, \Omega_{2}^{i}, \ldots, \Omega_{K}^{i}\right]$. It should be noted here that $\boldsymbol{\Omega}_{v}^{i}$ is independent of $n$ and $E\left(\left|s_{k}(q)\right|^{2} \mid v_{k}^{i-1}(q)\right)=1$.

The conditional MSE of the current iteration $i$, given the outputs from the previous iteration, is given by:

$$
\vec{J}^{i}=\frac{1}{B^{*} K} \sum_{n=0}^{B-1} E\left(t r\left(\left(\mathrm{~V}^{i}[n]-\mathrm{S}[n]\right)\left(\mathrm{V}^{i}[n]-\mathrm{S}[n]\right)^{\mathrm{H}}\right)\right)
$$

The conditional MSE (5.13) is to be minimized with respect to the forward filter $\hat{\mathbf{W}}^{i}[n]$ and feedback coefficients $\hat{\mathbf{F}}^{i}[n]$, subject to the constraint that the gain of the channel plus the forward filter for the $n$th frequency sample is to be equal to unity. Let $\mathbf{m}^{i}[n]=\operatorname{diag}\left(\mathbf{W}^{i^{\mathrm{H}}}[n] \mathbf{H}[n]\right)$. Then the constraint is equivalent to 


$$
\frac{1}{B} \sum_{n=0}^{B-1} m^{i}[n]=(1,1,1, \ldots, 1)^{*}
$$

This constraint is accounted for by use of a $K$-dimensional Lagrange multiplier vector:

$$
\vec{\mu}^{i}=\left[\mu_{1}^{i}, \mu_{2}^{i}, \ldots, \mu_{K}^{i}\right]
$$

where, $\mu_{k}^{i}=-\frac{\sum_{n=0}^{B-1} a_{k}^{i}[n]}{\sum_{n=0}^{B-1} \beta_{k}^{i}[n]}$

$\beta_{k}^{i}[n]=$ the kth element of $\operatorname{diag}\left(\hat{\mathbf{H}}[n]^{\mathrm{H}}\left[\hat{\mathbf{H}}[n] \overrightarrow{\mathbf{\Omega}}^{i}[n] \hat{\mathbf{H}}[n]^{\mathrm{H}}+\sigma_{n}{ }^{2} \mathbf{I}_{M}\right]^{-1} \hat{\mathbf{H}}[n]\right)$

$a_{k}^{i}[n]=1-\Omega_{v, k}^{i}[n] \beta_{k}^{i}[n]$

where $\Omega_{v, k}^{i}$ is the $k$ th element of the vector $\boldsymbol{\Omega}_{v}^{i}$. The update for the frequency domain feed forward equalizer's coefficients $\hat{\mathbf{W}}^{i}[n]$ are:

$$
\hat{\mathbf{W}}^{i}[n]=\left[\hat{\mathbf{H}}[n] \overrightarrow{\mathbf{\Omega}}^{i}[n] \hat{\mathbf{H}}[n]^{\mathrm{H}}+\sigma_{n}^{2} \mathbf{I}_{M}\right]^{-1} \hat{\mathbf{H}}[n]\left[\mathbf{\Omega}_{v}^{i}-\vec{\mu}^{i}\right]
$$

The frequency domain feed back equalizer coefficients $\hat{\mathbf{F}}^{i}[n]$ are given by:

$$
\hat{\mathbf{F}}^{i}[n]=\mathbf{I}_{K}-\hat{\mathbf{H}}[n]^{\mathrm{H}} \hat{\mathbf{W}}^{i}[n]
$$

The SD-IBDFE output at iteration $i>0$ is given by:

$$
\mathbf{V}^{i}[n]=\hat{\mathbf{W}}^{i}[n]^{\mathrm{H}} \mathbf{Y}[n]+\hat{\mathbf{F}}^{i}[n]^{\mathrm{H}} \hat{\mathbf{S}}^{i-1}[n]
$$

where,

$$
\hat{\mathbf{S}}^{i-1}[n]=\mathrm{F}_{B}\left(\hat{\mathbf{s}}^{i-1}(q)\right)
$$

Equalizer output samples:

$$
\mathbf{v}^{i}(q)=\mathbf{F}_{B}^{-1}\left(\mathbf{V}^{i}[n]\right)
$$

The corresponding conditional MSE is:

$$
\bar{J}^{i}=\frac{1}{B^{*} K} \sum_{n=0}^{B-1} \operatorname{tr}\left[\left(\hat{\mathbf{W}}^{i}[n]^{\mathrm{H}} \hat{\mathbf{H}}[n]-\mathbf{I}_{K}\right) \overrightarrow{\boldsymbol{\Omega}}^{i}\left(\hat{\mathbf{W}}^{i}[n]^{\mathrm{H}} \hat{\mathbf{H}}[n]-\mathbf{I}_{K}\right)^{\mathrm{H}}+\sigma_{n}^{2} \hat{\mathbf{W}}^{i}[n] \hat{\mathbf{W}}^{i}[n]^{\mathrm{H}}\right]
$$

The new soft decisions for the current iteration $i$ are obtained by using (5.10). Note that from (5.14) and the constraint (5.15), the following is true:

$$
\frac{1}{B} \sum_{m=0}^{B-1} \operatorname{diag}\left(\hat{\mathbf{F}}^{i}[n]\right)=[0,0, \ldots, 0]^{*}
$$


This implies that the zeroth feedback coefficient in the time domain is zero, and hence any soft decision $\hat{\mathbf{s}}^{i}(q)$ is not affected by $\hat{\mathbf{s}}^{i-1}(q)$.

Although the performance of the iterative receiver depends on the accuracy with which the time-varying CFR $\hat{\mathbf{H}}$ is estimated, it is worth noting that the soft decision feedback of SD-IBDFE results incurs less harmful error propagation effects than conventional hard decision feedback equalization. This SD-IBDFE has ability to mitigate ISI and suppress the in-cell interference as well as it offers a low complexity solution to the DFE problem. Notice within this SD-IBDFE receiver the equalization and channel decoding procedures are performed separately; that is the feedback loop uses the equalizer outputs instead of the channel decoder output. Higher performance gains can be achieved if these procedures are performed jointly. This is done by employing frequency domain Turbo Equalization (TE) schemes [TKS02] where the equalization and decoding processes are repeated in an iterative way. Receiver processing with frequency domain-TE is presented in Chapter 7.

\subsection{Performance Evaluation}

To simulate and evaluate the performance of the proposed SD-IBDFE we use the parameters and assumptions described in Table 3.1 (Chapter 3). The channel model and the corresponding average power delay profile are given in Table 2.1 (Chapter 2).

\subsubsection{SD-IBDFE Performance Evaluation with no Intra-cell Interference}

Improvement in average Frame Error Rate (FER) performance with SD-IBDFE SISO link $(M=K=1)$ is depicted in Figure 5.2. Each transmitted signal arrives at each receiving antenna through an independent $\mathrm{C} 2$ channel with a vehicle speed of $50 \mathrm{~km} / \mathrm{hr}$. The results are obtained after performing the Linear Equalization ( $\operatorname{LE}-I_{E}=0$, where $I_{\mathrm{E}}$ represents the total number of SD-IBDFE iterations), 1 and 4 SD-IBDFE iterations ( $I_{E}=1$ and 4). In these simulations, the length of the short training block $\left(T_{r}\right)$ is 104, while the block length $(B)$ is 416 and the simulations are carried out with 1000 channel realizations. Frequency 
Multiplexed Training (FMT) is used to multiplex the pilots among the data symbols. Notice that most of the gain is obtained in the first iteration $\left(I_{E}=1\right)$ and the gain gap reduces as we increase the number of SD-IBDFE iterations.

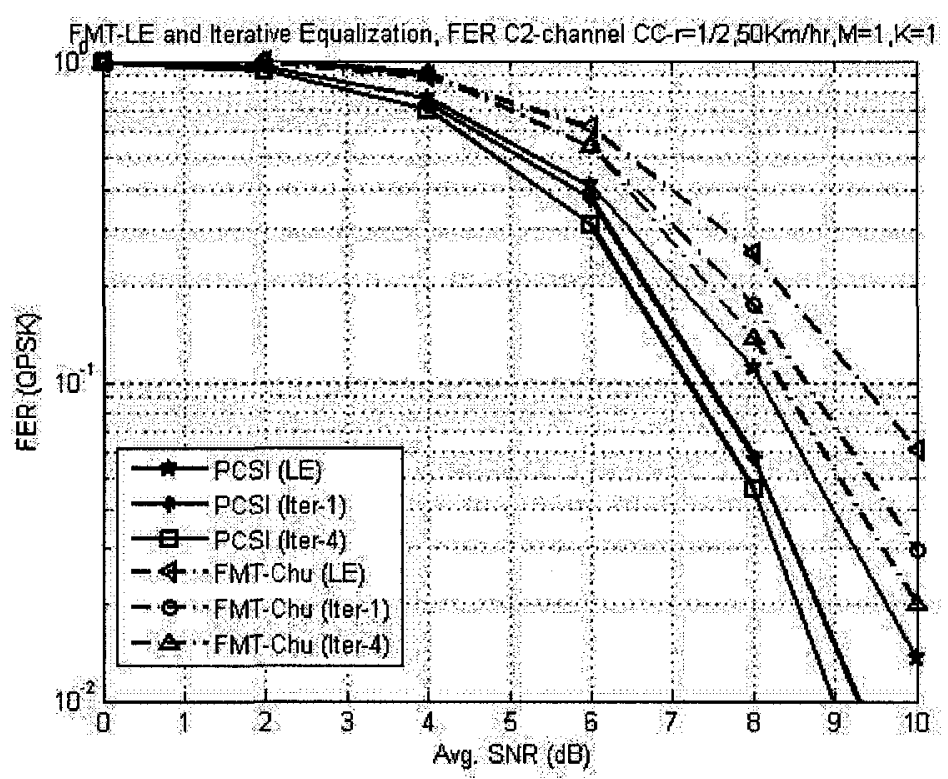

Fig. 5.2. PCSI Vs FMT-Chu, improvement in average FER through SD-IBDFE, $M=K=1$.

\subsubsection{SD-IBDFE Performance Evaluation with Intra-cell Interference}

In this section we evaluate the SD-IBDFE performance by considering the inclusion of the orthogonal co-channel ICUs. Figure 5.3 illustrates the performance comparison of SD-IBDFE with LE with two co-channel ICUs $(K=2)$. In this case the three possible orthogonal training options are FMT, TMT-ortho and TMT-Al (only with $K=2$ ). In FMT scheme, pilots are orthogonally inserted in the data block in a frequency-multiplexed manner. TMT-ortho represents pilot multiplexing in time by using short training blocks. The TMT-Al produces the orthogonal training via Alamouti space-time coding and is only possible with two transmitting sources $(K=2)$.

With PCSI, an improvement of around $1.8 \mathrm{~dB}$ is achieved over LE, using SD-IBDFE algorithm with $I_{E}=4$. With CE and two antenna elements $(M=2)$ at the BS, the SNR gain of about 1.2-2.0 dB is obtained with SD-IBDFE. An independent C2-channel for each transmitter-receiver link with a mobility of $50 \mathrm{~km} / \mathrm{hr}$ is used in the simulations. Two short 
training blocks are used. 2000 channel realizations are considered to obtain the average BER results.

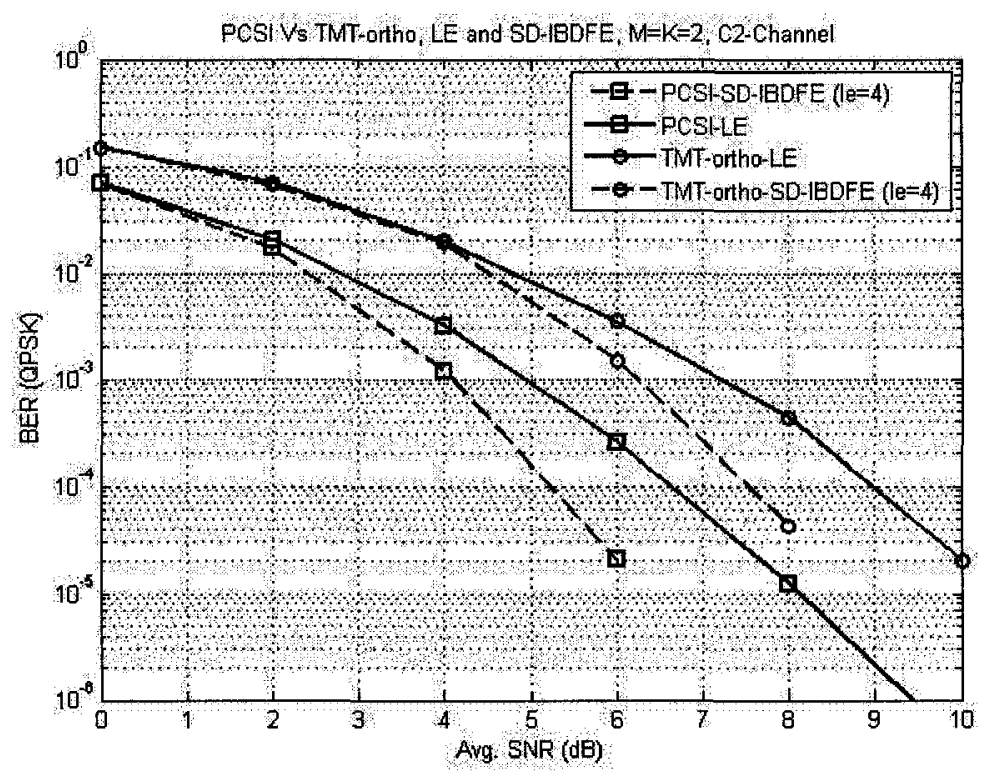

Fig. 5.3. Average BER comparison between $\operatorname{LE}\left(I_{\mathrm{E}}=0\right)$ and SD-IBDFE $\left(I_{\mathrm{E}}=4\right), M=K=2$.

\subsubsection{SD-IBDFE Performance Evaluation with Intra and Inter-cell}

\section{Interference}

In order to see the performance of the SD-IBDFE with in-cell as well as out of cell interference we have used a scenario described in Section 4.4.3 for the uncompensated OCIs with Intra-cell Interference. Figure 4.11, reproduced here as Figure 5.4 for convenience depicts the specific interference distribution. In this case, there is a loadsharing situation in which one half of the OCIs' training overlaps one of the ICU and the other half of the OCIs overlaps the other ICU.

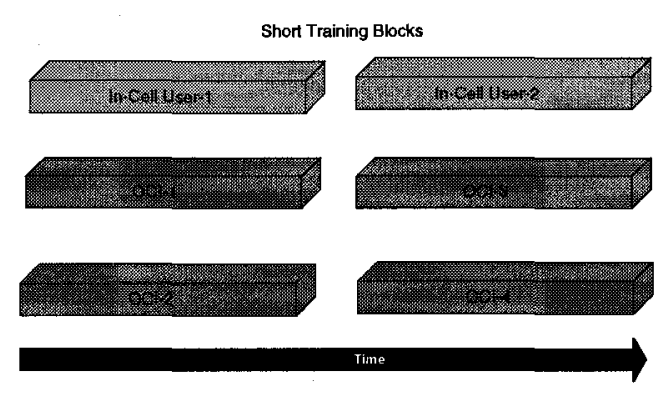

Fig. 5.4. Scenario of the overlapping OCIs' and ICUs' training blocks. 
In evaluation illustrated in Figure 5.5, we have used the modified simulation parameters described in Table 4.1. 2000 channel realizations are used in order to obtain the curves. There are two orthogonal ICUs in the system and two OCIs are affecting each ICU. The relative powers of the interfering OCIs are $6 \mathrm{~dB}$ below the ICUs. Simulations are carried out with four antenna elements at the BS. The number of short training blocks $(N T)$ is equal to the total number of users $(K+P)$. It is evident that at higher SNRs, SD-IBDFE based iterative processing further improves the average BER performance by around 1.4 and more $\mathrm{dBs}$. This suggests that the soft cancellation provided by the SD-IBDFE could be a good candidate for suppressing the co-channel interference along with the ISI. The Iterative Equalization (iter\#4) in the legend shows 4 SD-IBDFE cycles $\left(I_{E}=4\right)$, using initial channel estimates derived from pilots and 2x1D Wiener interpolation.

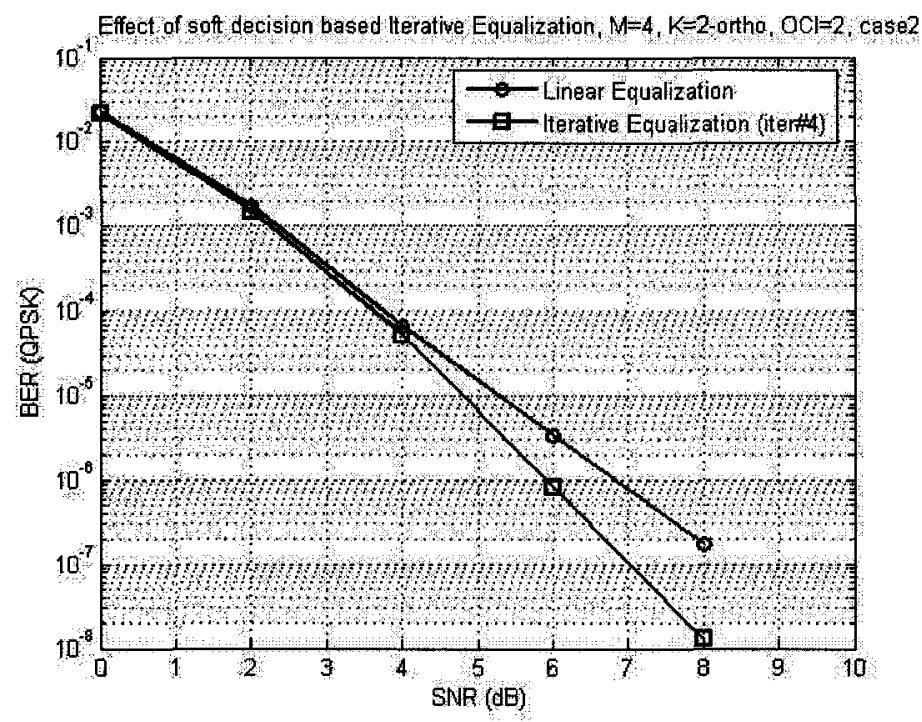

Fig. 5.5. Average BER-Improvement by SD-IBDFE (Uncompensated OCIs with intracell interference), $M=4, K=2, P=2+2$.

\subsection{Summary}

In this chapter we propose a new low complexity block-based SD-IBDFE, which assists to remove ISI and suppresses the co-channel interference. As the feedback coefficients update involves soft decisions, the possibility of error propagation at higher SNR is reduced. As both feed forward and feedback filters are employed with efficient FFT implementation, the processing complexity is much lower compared to the DFEs based 
on the time domain feedback processing, especially in the case of larger channel memory. This gives a notion that with improved channel estimates via any decision-directed approach, the proposed SD-IBDFE has ability to further suppress the interference. Moreover, as SD-IBDFE computes and updates the soft decisions, it would be possible to easily modify it to accommodate the Turbo Equalization (TE). 


\section{Chapter 6}

\section{Interference Suppression in SC-FDE SDMA}

\section{System}

\subsection{Introduction}

This chapter focuses on developing and evaluating algorithms to suppress the intra-cell interference as well as the inter-cell interference caused by the uncompensated OCIs (unknown interferences). The chapter consists of three parts: The first part proposes and evaluates a new SC-FDE multi-user hard decision-based threshold-based Decision Feedback Iterative Channel Estimation (HD-DFICE) algorithm. The second section is dedicated to the $\mathrm{CE}$ improvement in the presence of intra and inter-cell interference via a version of PIC-assisted HD-DFICE. In the third section, a Least Squares (LS) forward filter processing is proposed to further mitigate the interference.

Also, iterative CE over multiple frames to estimate the desired user's channels provides some additional gain. Simulation results show that the combination of these low complexity algorithms gives reasonably good results in interference-dominated doubly selective channels.

\subsection{Decision Feedback Iterative Channel Estimation (DFICE)}

This is an era of iterative receiver processing. A popular estimation technique integrated in an iterative receiver is Decision Feedback Iterative Channel Estimation (DFICE). Iterative Channel Estimation (ICE) for mobile wireless channels has received great attention due to their performance gain [SJS03]. In DFICE, both pilot symbols and soft or hard decisions (estimates of the data symbols) are used to improve the channel estimate quality in a semi-blind manner [SLS98]. In the DFICE, in contrast to purely pilot assisted CE methods [Li00] both the pilot symbols as well as the decisions on the information symbols are utilized for CE. The simple notion of the method is that in the absence of 
transmission errors we can benefit from the availability of $100 \%$ pilot information by using the detected symbols as an a-posteriori reference signal. It should be noted that the decision-directed CE algorithms face error propagation at lower SNRs.

\subsubsection{Overview of the DFICE}

[SLS98] presents initial work on ICE in a GSM-like system, and showed performance gain with soft decision feedback. Soft decisions improve the quality of the channel estimates due to less error propagation. More recently, a series of work on SISO-DFICE with MC-CDMA and OFDM system is done in [SRA05], [BA06] and [BA07]. A recent SISO CE using DFICE in SC-FDE system is presented in [LFD08]. It should be noticed that in the presence of inter-user interference the decision-directed approaches become complex and more vulnerable to the error propagation due to the overlapping data of different co-channel users. There is an abundance of research done on ICE with MIMOOFDM systems [CL05], [ZSW06] and [QYS05]. A DFICE, has been presented for the space-time trellis coded (STTrC) OFDM system in [LSA99]. A similar idea has been

generalized to the MIMO-OFDM system in [LWS02]. Due to the high complexity of the matrix inversion encountered in the LS and MMSE estimator, several reduced complexity $\mathrm{CE}$ algorithms, for the MIMO-OFDM system have been proposed [Li02], [XG03] and [LWL02]. Most of the previous work on interference in MIMO-OFDM-DFICE systems involve turbo processing and will be described later in Chapter 7.

\subsubsection{HD-DFICE Algorithm}

The CFR estimates used in the previous chapters are computed from a combination of known pilots and the 2x1D Wiener interpolation filter. Also a Low Pass (LP) smoothing filter is used to further suppress the effect of noise. The CFR estimates of the ICUs can further be improved iteratively by making use of the decisions. These decisions are taken either before the decoding stage in the receiver or coding gain can also be utilized by picking up the decisions after the decoder. In this work we include the coding gain while making the decisions. In this section we will introduce a threshold-based hard decision- 
based DFICE (HD-DFICE). A simple block diagram of the proposed multi-user system employing the HD-DFICE is shown in Figure 6.1.

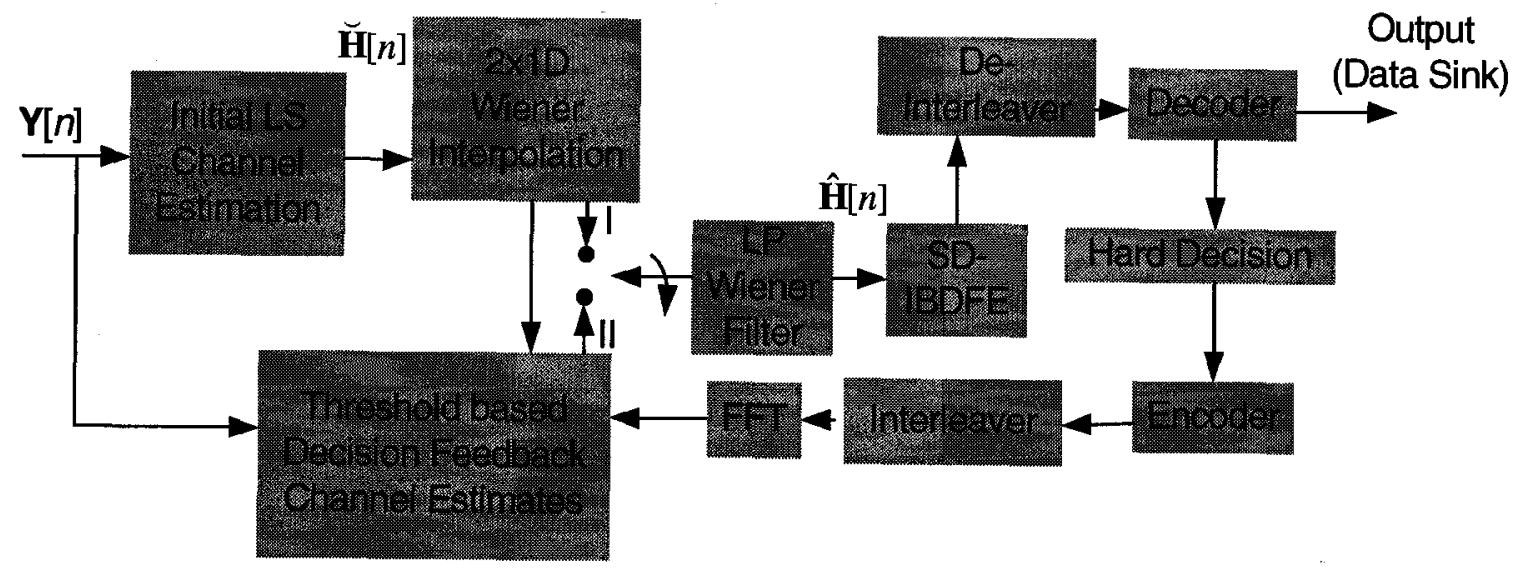

Fig. 6.1. Block diagram of hard decision-based CE (HD-DFICE).

Low Pass (LP) smoothing filter obtains the initial estimates through the position I in Figure 6.1. In LP filtering, the full-length estimated CFR is converted into the time domain by using an IFFT and smoothing is done over the noise caused by the AWGN and the unknown OCIs by setting the IR samples beyond the maximum span of $L$ to zero, where $L$ represents the channel memory. After passing through the iterative equalization (SD-IBDFE) and the Viterbi decoder, hard decisions are available at the receiver. The message information is fed back to the encoder and an interleaver. At this point, decisions on all frequencies become available. An FFT is performed on them and an updated channel estimates for the $k$ th ICUs at $n$th frequency and $j$ th HD-DFICE iteration for $j=1,2, \ldots, I_{\mathrm{ICE}}$ are computed as:

$$
\hat{\mathbf{H}}_{k}^{j}[n]=\frac{\mathbf{Y}[n]}{\tilde{S}_{k}^{j}[n]}
$$

where $I_{\text {ICE }}$ represents the total number of DFICE iterations, $\mathrm{Y}[n] \in C^{M \times 1}$ is the array input and $\tilde{S}_{k}^{j}[n]$ represents the hard decision for the $k$ th ICU after $j$ HD-DFICE iterations at the $n$th frequency such that $n=0,1, \ldots, B-1$. It should be noted that these hard decisions are obtained after several SD-IBDFE iterations $\left(i=0,1, \ldots, I_{\mathrm{E}}-1\right.$, typically $\left.I_{\mathrm{E}}=4\right)$ and the decoder stage. 
In SC-FDE, while invoking (6.1) a possible problem is noise enhancement at frequencies where $\tilde{S}_{k}^{j}[n]$ for $k$ th user has a smaller magnitude. In other words, as the envelope of the frequency response of the decisions is not constant in SC-FDE, there is an issue of noise enhancement while computing the CFR via decisions. This issue is tackled by using a threshold-based Frequency Replacement Technique (FRT) in the HD-DFICE. In FRT the noise enhancement affected CFRs are replaced by either the CFRs obtained in the previous ICE iteration i.e. $\hat{\mathbf{H}}_{k}^{j-1}$ or with the pilot-based initial CFRs i.e. $\hat{\mathbf{H}}_{k}^{0}$. A rationale for the FRT is provided by (7.24) in Chapter 7. An empirically chosen threshold $\lambda$ is set to decide the reliability of the decision, thus if $\left|\tilde{S}_{k}^{j}[n]\right|$ is less than the threshold $\lambda$, we write the estimate update as:

$$
\hat{\mathbf{H}}_{k}^{j}[n]=\left\{\begin{array}{l}
\hat{\mathbf{H}}_{k}^{j-1}[n] \ldots \ldots \ldots \ldots \ldots \ldots \ldots . . . \mathrm{if}\left|\tilde{S}_{k}^{j}[n]\right|<\lambda \\
\hat{\mathbf{H}}_{k}^{j}[n]=\frac{\mathbf{Y}[n]}{\tilde{S}_{k}^{j}[n]} \ldots \ldots \ldots . . . \text { Otherwise }
\end{array}\right.
$$

In Figure 6.1, the input of the LP filter after first HD-DFICE iteration $(j=1)$ is taken from position II. After first HD-DFICE iteration, $\hat{\mathbf{H}}_{k}^{j}[n]$ obtained via (6.1) is fed back to the SD-IBDFE that uses the updated CFR estimates for its internal iterations $\left(i=0,1, \ldots, I_{\mathrm{E}^{-}}\right.$, typically $\left.I_{\mathrm{E}}=4\right)$. Iterative equalizer output is again fed to the decoder and an update of the hard decisions is available for the next HD-DFICE iteration and so on.

The employment of the proposed multi-user HD-DFICE allows us to reduce the number of required pilot symbols to achieve a certain FER performance. It is shown in the later sections of the chapter, where we evaluate interference-prone systems, that the amount of the training (number of short training blocks $N T$ ) that dictates the pilot overhead can significantly be reduced with the help of HD-DFICE at the cost of slightly increased computational complexity.

\subsection{Simulation Results}

To evaluate the performance of the proposed HD-DFICE algorithm we used the parameters and assumptions described in Table 4.1 (Chapter 4). The channel model and the corresponding average power delay profile are given in Table 2.1 (Chapter 2). 
It should be noted that the simulations include any error propagation effects resulting from feedback of incorrect hard decisions.

\subsubsection{HD-DFICE Performance Evaluation with no Multi-User \\ Interference}

Figure 6.2 compares the average BER results with LE, SD-IBDFE and the HD-DFICE with SISO link. For reference purposes PCSI curves are also shown. In these simulations, the number of pilots spread in the first and last data block is 256. Equalizer output is obtained after 4 iterations of SD-IBDFE $\left(I_{E}=4\right)$. Output of the SD-IBDFE is deinterleaved, and after passing through a Viterbi decoder and convolutional encoder, the code bits are again interleaved. By using a simulation-based threshold of $\lambda=13$, channel estimates are computed iteratively. Each transmitted signal arrives at each receiving antenna through an independent $\mathrm{C} 2$ channel with a vehicle speed of $50 \mathrm{~km} / \mathrm{hr}$. A gain of about $1.2 \mathrm{~dB}$ is observed with HD-DFICE over the LE. Results shown in Figure 6.2 are after one HD-DFICE iteration $\left(I_{I C E}=1\right)$. It should be noted that each $I_{I C E}$ includes all $I_{E}$ i.e. each $I_{I C E}$ includes 4 SD-IBDFE iterations $\left(I_{E}=4\right)$.

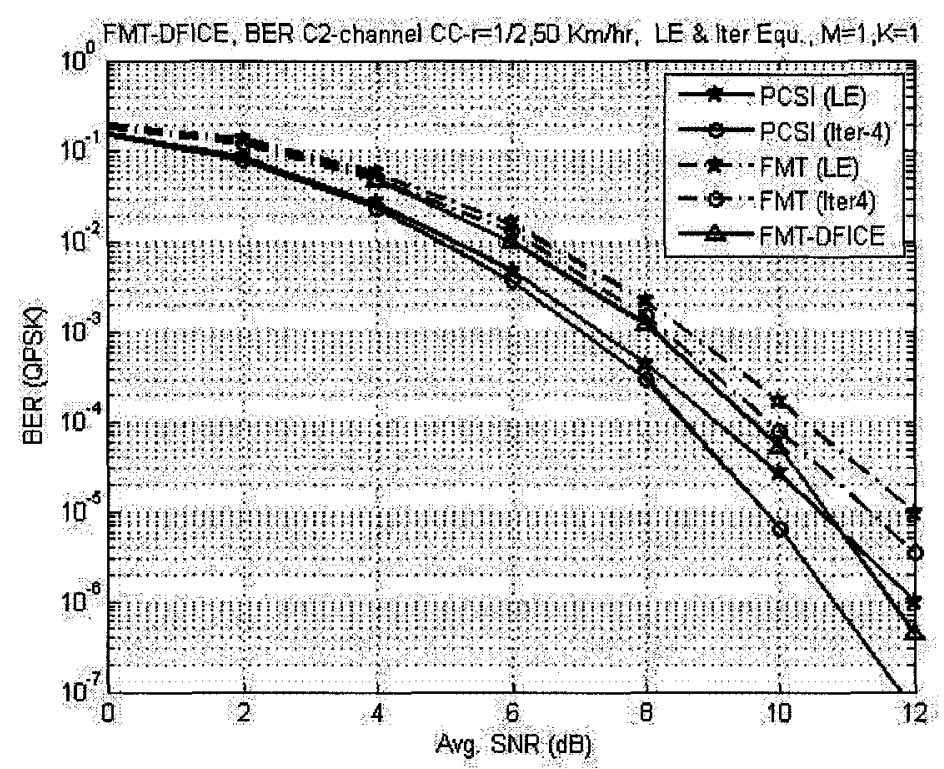

Fig. 6.2. Improvement in average BER via $\mathrm{HD}$-DFICE, $M=K=I_{I C E}=1, I_{E}=4, \mathrm{C} 2-\mathrm{Channel}$, $50 \mathrm{~km} / \mathrm{hr}, N T=1$, results with LE and SD-IBDFE also shown for comparison. 


\subsubsection{HD-DFICE Performance Evaluation with Inter-Cell Interference}

It is illustrated in the previous section that with HD-DFICE based SISO system BER performance can be improved by around $1 \mathrm{~dB}$ (See Figure 6.2). In this section, the HDDFICE is applied to a scenario with no intra-cell interference, but having unknown (uncompensated) OCIs. There is only one ICU of interest and the focus is on the CE aspects of the HD-DFICE algorithm in the presence of the interference caused by several OCIs.

Figures 6.3 and 6.4 illustrate the average MSE at the equalizer output and the average FER results respectively with the employment of the HD-DFICE with one ICU $(K=1)$ and two or three OCIs ( $P=2$ and 3$)$. The average MSE is computed using (5.20). The relative powers of the uncompensated OCIs are assumed to be $15 \mathrm{~dB}$ lower than that of ICU. Two antenna elements $(M=2)$ at the BS are used. The number of short training blocks sent by the ICU prior to the first and last block of the frame is two $(N T=2)$. This means that a total of 4 short blocks per ICU are sent in one frame.

Average MSE performances of pilot-supported LE, pilot-supported SD-IBDFE with $I_{E}=4$, and HD-DFICE-supported SD-IBDFE, with 2 or 3 OCIs, with $I_{I C E}=1$, are compared in Figure 6.3. The crossover at lower SNRs is caused by the error propagation in both SDIBDFE and HD-DFICE. The SD-IBDFE (iter4) in the legend represents one SD-IBDFE cycle with $I_{E}=4$, using only initial CFR estimates derived from pilots. Similarly, the HDDFICE (iter1) means two SD-IBDFE cycles with $I_{E}=4$ each, with second cycle using updated ICE-derived CFR estimates. The notation "nk" means that the desired BS does not have knowledge about the training sequences of the OCIs.

Figure 6.4 illustrates the corresponding average FER. It should be noted that, as the number of OCIs increases, the inter-cell interference makes the array input noisier and the performance of HD-DFICE is expected to deteriorate (as depicted in the FER results). Average FER with PCSI is also plotted for reference purposes. A similar trend is expected with the presence of higher power OCIs. With further increase in the number of OCIs or their relative powers, the performance of HD-DFICE might deteriorate, but will still be better than LE or SD-IBDFE at higher SNR values. Presence of Intra-cell interference will further affect the performance of all these algorithms. Performance of the HD-DFICE in the presence of intra-cell interference is addressed in Section 6.4. 


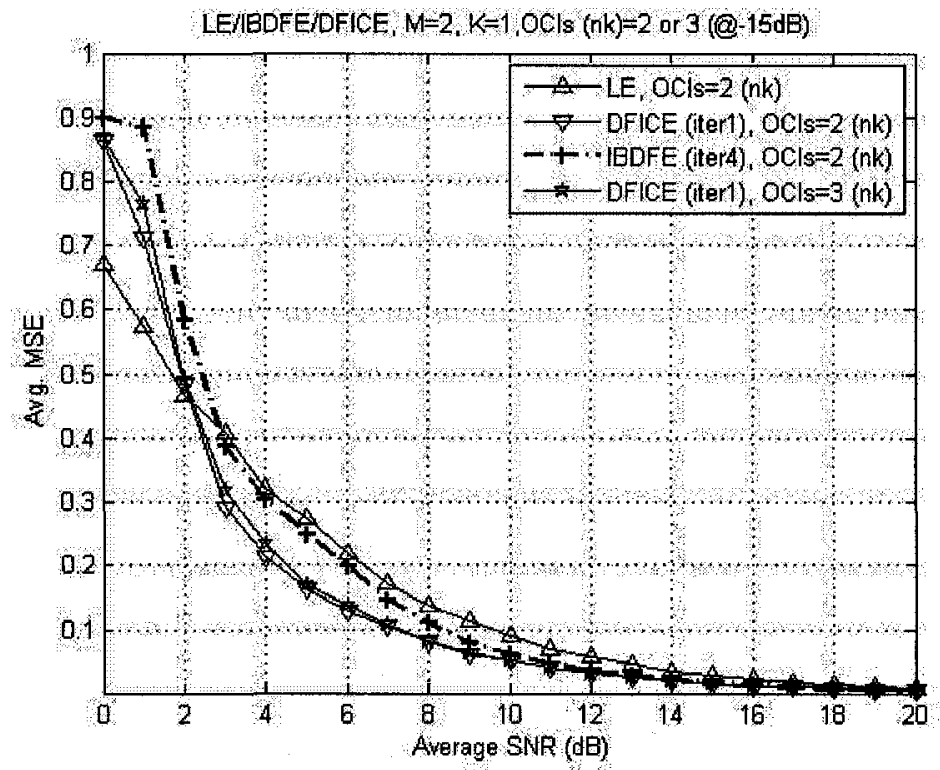

Fig. 6.3. Average MSE with only LE, SD-IBDFE and SD-IBDFE with HD-DFICE

(Uncompensated OCIs no Intra-cell interference) $M=2, K=I_{I C E}=1, I_{E}=4, P=2$ or $3 @-15 \mathrm{~dB}$, C2-Channel, $50 \mathrm{~km} / \mathrm{hr}, N T=2$.

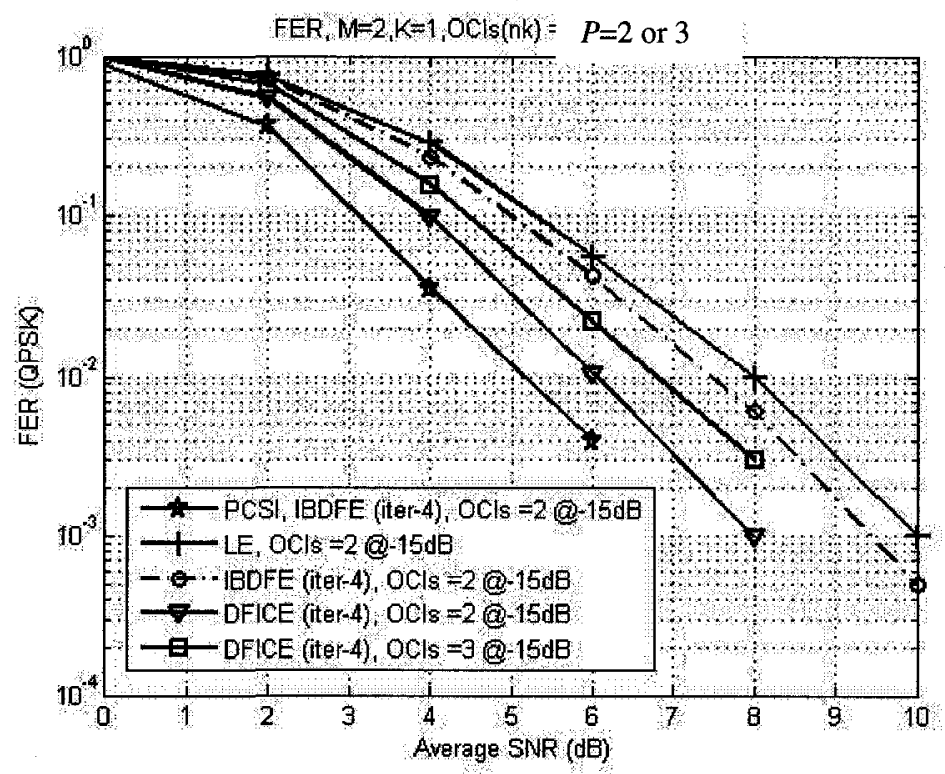

Fig. 6.4. Average FER with only LE, SD-IBDFE and SD-IBDFE with HD-DFICE (uncompensated OCIs no intra-cell interference) $M=2, K=I_{I C E}=1, I_{E}=4, P=2$ or $3 @-15 \mathrm{~dB}$, C2-Channel, $50 \mathrm{~km} / \mathrm{hr}, N T=2$. 
Figure 6.5 depicts the average FER as a function of the number of OCIs. There is only one ICU $(K=1)$, two receive antenna elements $(M=2)$, the average SNR at each antenna element is $6 \mathrm{~dB}$ and the OCIs' received average power is either $-15 \mathrm{~dB}$ or $-16.6 \mathrm{~dB}$. C2 channel with a vehicle speed of $50 \mathrm{~km} / \mathrm{hr}$ is used. OCIs interfere with ICU data and pilot signals, and as mentioned above, there is no attempt to compensate for the OCIs. The number of training blocks is $2(N T=2)$. Following equalization algorithms at the receiver are compared:

- Linear Equalization (LE) with ICU CFR estimated from pilot-aided CE (PACE) with $2 \times 1 D$ Wiener interpolation.

- SD-IBDFE with ICU CFR initially estimated with PACE and in subsequent iterations by HD-DFICE.

It is observed from Figure 6.5 that for an average FER of $10^{-2}$, only one $-16.6 \mathrm{~dB}$ OCI can be tolerated by the $\mathrm{LE}$, while two $-15 \mathrm{~dB}$ or four $-16.6 \mathrm{~dB}$ OCIs can be tolerated by the HD-DFICE-assisted SD-IBDFE. The results demonstrate the effectiveness of HDDFICE. They also show the system's sensitivity to the number of uncompensated OCIs, especially for a fixed amount of pilots and also to the number of receiving antenna elements that is less than the total number of interferers $(M<K+P)$.

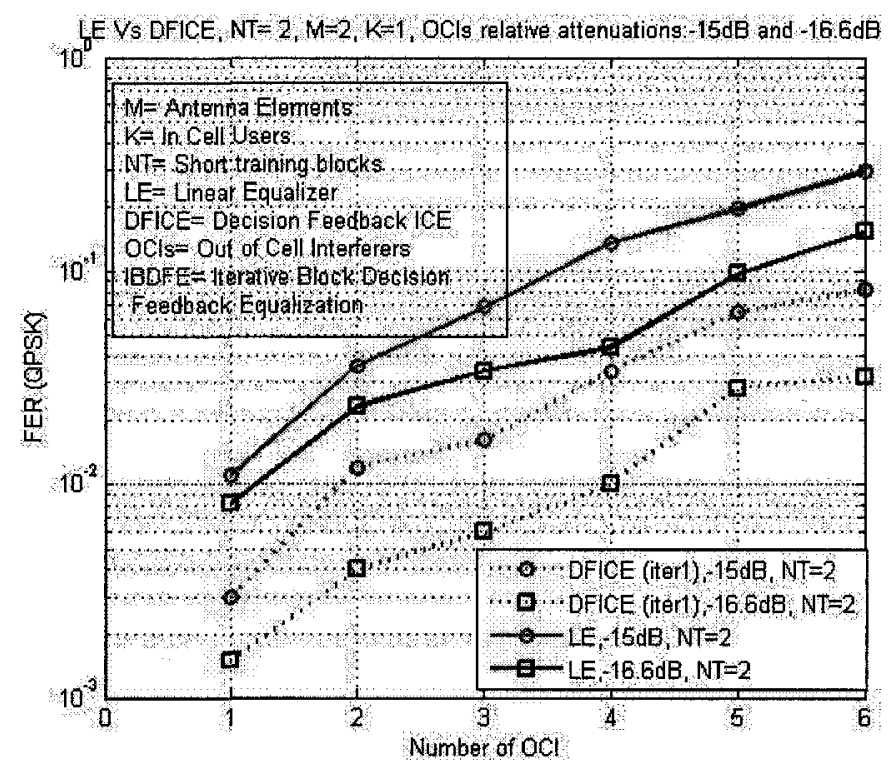

Fig. 6.5. Average FER with only LE and SD-IBDFE with HD-DFICE (uncompensated OCIs with no intra-cell interference @- $15 \mathrm{~dB}$ or $-16.6 \mathrm{~dB}) M=2, K=I_{I C E}=1, I_{E}=4$, C2-

Channel, $50 \mathrm{~km} / \mathrm{hr}, N T=2$. 
Average BER results shown in Figure 6.6 are obtained with fixed number of training blocks $(N T=2)$ and also with the training blocks equal to the sum of in-cell and out of cell users $(K+P)$. It shows less sensitivity to the number of OCIs and additional gain is achievable by sending more training blocks, but this would require a proportional increase in pilot overhead.

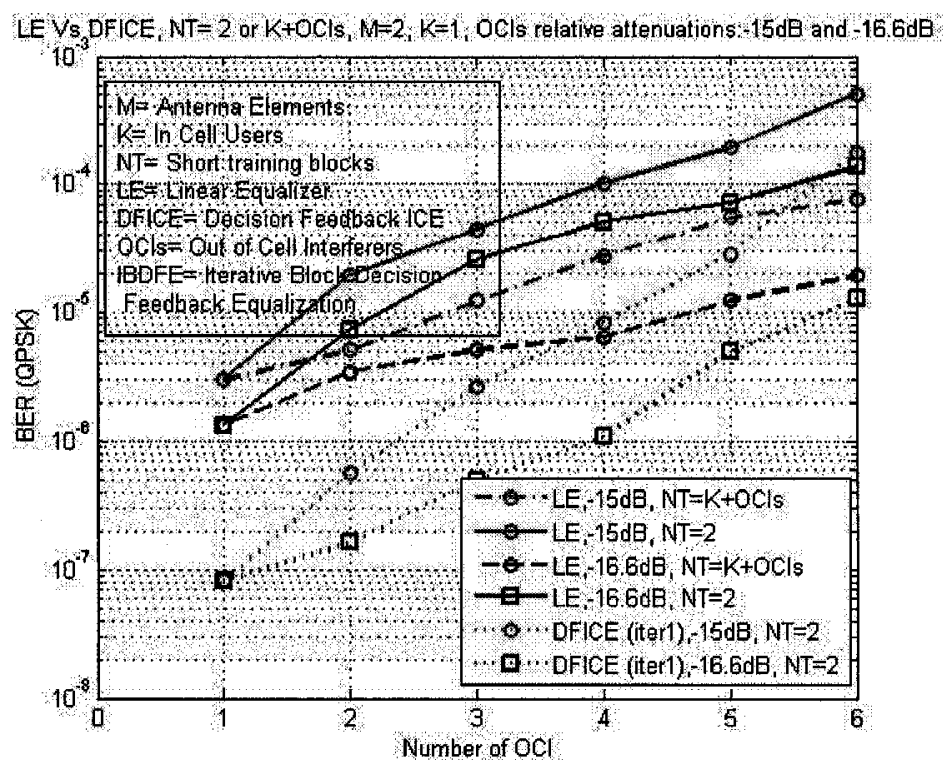

Fig. 6.6. Average BER with only LE and SD-IBDFE with HD-DFICE (uncompensated OCIs with no intra-cell interference $@-15 \mathrm{~dB}$ or $-16.6 \mathrm{~dB}), M=2, K=I_{I C E}=1, I_{E}=4, \mathrm{C} 2$ -

Channel, $50 \mathrm{~km} / \mathrm{hr}, N T=2$ or $K+P$.

\subsection{Parallel Interference Cancellation (PIC) assisted HD- DFICE}

In the previous section, we evaluated the performance of the HD-DFICE with inter-cell interference in which the power levels of the OCIs are much lower than the ICU. In case of intra-cell interference we face relatively higher power or, due to slow power control, equal power interferers. In the presence of intra-cell interference the decision-directed $\mathrm{CE}$ approaches become sensitive to multiple user fed back decision errors. To utilize the proposed HD-DFICE in the presence of the intra-cell interference along with the inter- 
cell interference, the array input should be as much interference-free as possible. This is accomplished by using a well-known process called Parallel Interference Cancellation (PIC).

PIC is a promising way of mitigating the effects of co-channel multi-user interference or, more generally, interference in a SDMA system [MH00]. PIC detects and subtracts the estimates of all interfering signals in parallel before detection of the wanted signal. The obtained interference is subtracted, i.e. canceled from the received signal, and the data detection is performed again with reduced multiple access interference. In ICE the subtracted interference version of the array input is used to obtain the new channel estimates.

\subsubsection{Overview of the PIC}

PIC is not a new interference cancellation technique. The Successive Interference Cancellation (SIC) technique was first proposed for uplink OFDM by [VPE99], and PIC was proposed in the same paper as a future candidate for interference suppression in SDMA systems. SIC and PIC are compared in [SSM99] with coded multi-user OFDM system and used in combination with the other adaptation schemes in [MHOO] and [MH05]. With SC-FDE systems, PIC is used to suppress the interference with perfect channel knowledge in [KDF04].

\subsubsection{PIC-assisted HD-DFICE Algorithm}

As described earlier, PIC can potentially suppress the intra-cell interference, given estimates of intra-cell CFRs, and eventually the channel estimates can be further improved with a subsequent HD-DFICE. We propose an architecture, which combines the iterative equalization of SD-IBDFE, with PIC and HD-DFICE. HD-DFICE is fed with an improved array input of different ICUs filtered through PIC. Decoder hard decisions on interfering in-cell data (obtained after several SD-IBDFE iterations and Viterbi decoding) are used to remove estimated interference, and the HD-DFICE obtains the CFR estimates from the residual. 
PIC is invoked in a very simple way. At the $j$ th HD-DFICE iteration, for the $k$ th ICU, at the $n$th frequency, after $I_{E}$ iterations of SD-IBDFE, the cancellation from all other ICUs is done thusly:

$$
\mathbf{X}_{k}^{j}[n]=\mathbf{Y}[n]-\sum_{\substack{\ell=1, \ell \neq k}}^{K} \hat{\mathbf{H}}_{l}^{j-1}[n] \tilde{S}_{l}^{j}[n]
$$

where $\hat{\mathbf{H}}_{l}^{j-1}[n]$ represents the estimated CFR for the $l$ th user at $(j-1)$ th iteration at $n$th frequency, $\mathrm{Y}[n] \in \mathrm{C}^{M \times 1}$ is the array input having a composite signal from all the interfering sources and $\tilde{S}_{l}^{j}[n]$ represents the hard decision of $l$ th user after $j$ HD-DFICE iterations for $n=0,1, \ldots, B-1$. In the HD-DFICE unit, the updated CFR estimates for the $k$ th ICU are computed as:

$$
\hat{\mathbf{H}}_{k}^{j}=\frac{\mathbf{X}_{k}^{j}[n]}{\tilde{S}_{k}^{j}[n]}
$$

After getting updated CFR estimates via (6.4), a combination of frequency and time domain smoothing over successive FFT blocks as described in Chapter 2 is applied. As mentioned in Section 6.2.2, a possible problem when applying (6.4) is noise enhancement at frequencies $n$ where $\tilde{S}_{k}^{j}[n]$ has a small magnitude. This problem is dealt with by a threshold-based frequency replacement FRT method described in Section 6.2.2. Notice that PIC is sensitive to inaccurate subtraction of ICU interference due to ICU CE and hard decision errors.

\subsection{Simulation Results}

In order to simulate the proposed PIC assisted HD-DFICE algorithm, we use the parameters and assumptions described in Table 4.1 (Chapter 4) and the channel model and the corresponding average power delay profile are given in Table 2.1 (Chapter 2). Also the interference distribution scenario is the 'case 2' described in Section 4.4.3 in Chapter 4. 


\subsubsection{PIC-assisted HD-DFICE Performance Evaluation with Intra and Inter-cell Interference}

Two orthogonal ICUs $(K=2)$ with 4 OCIs $(P=4)$ are considered in the system (see Figure 4.11). 'Case 2' is a load-sharing situation wherein one half of the OCIs' training overlaps one of the ICU and the other half of the OCIs overlaps the other ICU (depicted as $2+2$ in Figure 6.7). Two OCIs' pilots interfere with each of the two ICUs' pilots, and each ICU's data is interfered with by the other ICU's data plus all 4 OCIs' data.

Each OCI has the same average received power, either $-15 \mathrm{~dB}$ or $-18.2 \mathrm{~dB}$. There are 4 receiving antenna elements $(M=4)$ at the BS. Each transmitted signal arrives at each receiving antenna through an independent $C 2$ channel with a vehicle speed of $50 \mathrm{~km} / \mathrm{hr}$.

Also shown are average FER curves with PCSI in two cases:

(1) The channels of all the in-cell and out of cell users are known, and a LE suppresses all the OCIs as well as the in-cell interferer (Labeled PCSI, LE, OCIs@-15dB).

(2) Only the channels of the ICUs are known (the OCIs still just add to the noise), and a LE receiver detects ICUs (Labeled PCSI, ICUs only (OCIs@-15dB)).

The OCIs are at $15 \mathrm{~dB}$ below the ICUs in each of these PCSI cases. With OCIs at this level, it is seen that knowing only the ICUs' channels causes about a $0.4 \mathrm{~dB}$ SNR penalty relative to the case where all channels are known and taken into account. Figure 6.7 also shows a substantial amount of SNR penalty relative to PCSI ( 4.5 to $5 \mathrm{~dB}$ ), for LE when the ICU channels are estimated non-iteratively. This penalty in evidently caused by inaccurate channel estimates which result from interference to pilots from noise and OCIs. LE performance of around 2.7dB SNR degradation with no Inter-cell interference (no OCI) is also shown. 


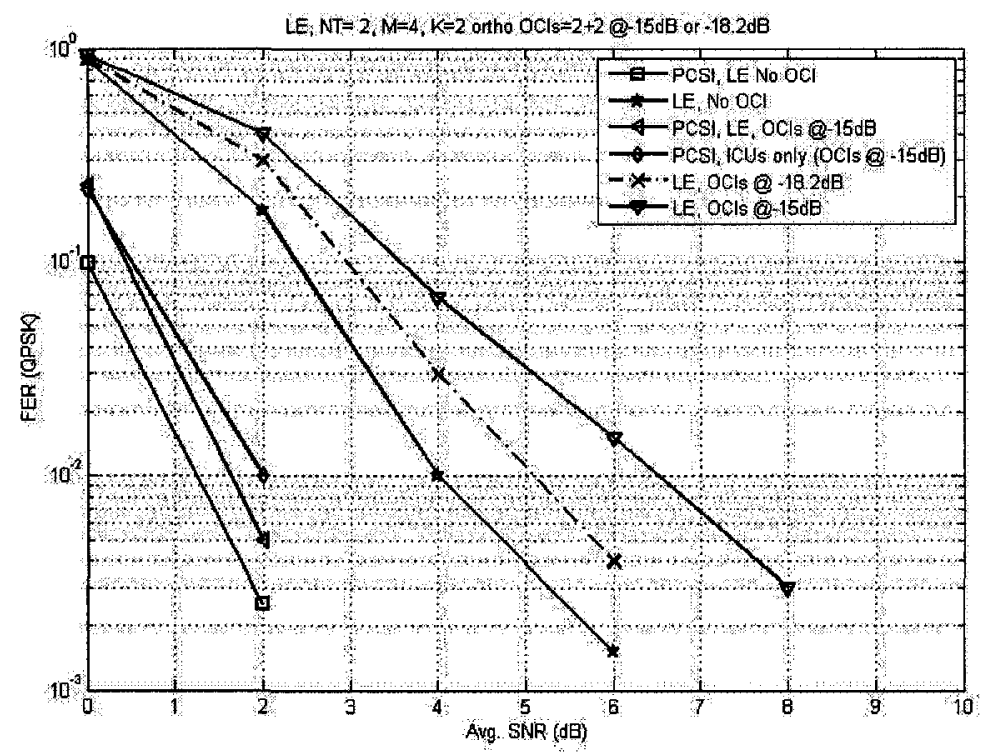

Fig. 6.7. Average FER with LE processing (uncompensated OCIs with inter and intra-cell interference), $M=4, K=2, \mathrm{C} 2-\mathrm{Channel,} 50 \mathrm{~km} / \mathrm{hr}, N T=2, P=4 @-15 \mathrm{~dB}$ and $-18.2 \mathrm{~dB}$.

Figure 6.8 shows average FER results, under the 'case2' interference condition as mentioned above. We evaluate and compare the performance of the following:

1- SD-IBDFE equalization with $I_{E}=4$ using initial pilot-based CFR estimates (Labeled 'IBDFE').

2- With the PIC assisted HD-DFICE supported SD-IBDFE with $I_{I C E}=1$ or 2 and each $I_{I C E}$ contains $I_{E}=4$ (Labeled 'DFICE').

There is an improvement over the LE, non-iterative CE case; the SNR penalty relative to perfect CSI is now about $3 \mathrm{~dB}$ at $10^{-2} \mathrm{FER}$, for the two OCIs per ICU at $-15 \mathrm{~dB}$. Figure 6.8 also shows the case where there is no OCI. The SNR penalty attributable to the presence of the two $-15 \mathrm{~dB}$ OCIs per ICU is about $1.5 \mathrm{~dB}$. The substantial ( $>2 \mathrm{~dB})$ SNR penalty relative to PCSI is due to the effect of noise and OCI interference on the channel estimates that are used in IBDFE equalization with PIC. 


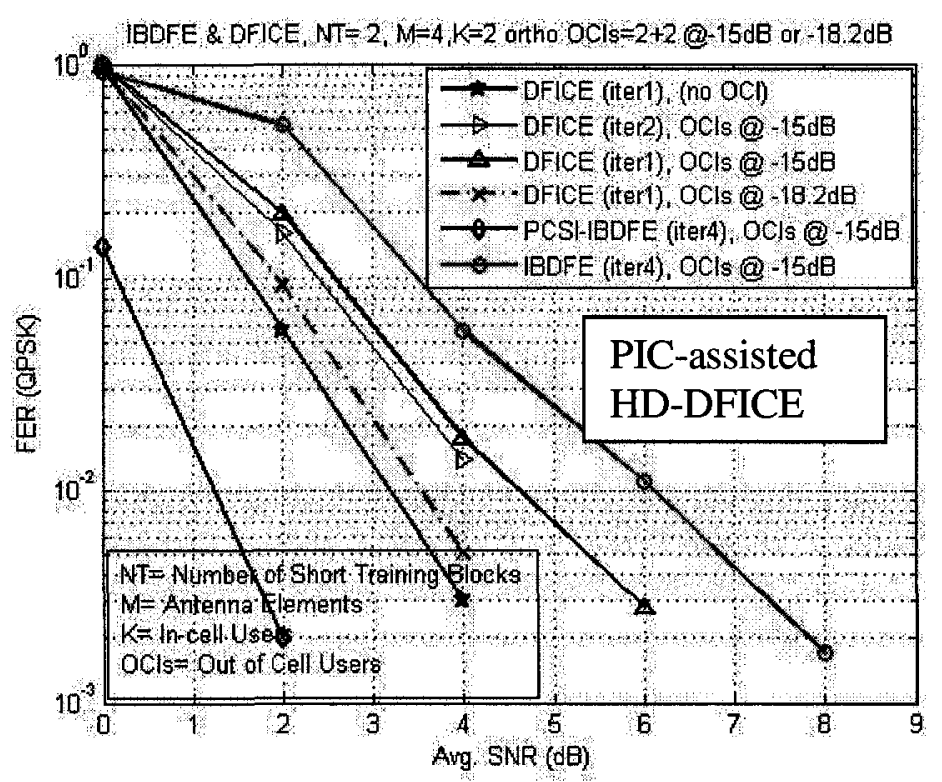

Fig. 6.8. Average FER with SD-IBDFE and PIC assisted HD-DFICE (uncompensated OCIs with inter and intra-cell interference), $M=4, K=2, \mathrm{C} 2$-Channel, $50 \mathrm{~km} / \mathrm{hr}, N T=2$, $P=4 @-15 \mathrm{~dB}$ and $-18.2 \mathrm{~dB}$.

\subsection{Interference Mitigation via LS-IS Algorithm}

In PIC-based HD-DFICE, the main emphasis was on suppressing the intra-cell interference by utilizing the hard decisions. We propose a further step in the attempt to remove the effects of low power OCIs on the CE without explicitly estimating their channels. Estimating the CFRs of the low power OCIs has two problems. First is that the receiver processing complexity would increase per OCI and secondly due to their low powers it is hard to reliably estimate their CFRs. The proposed solution involves the Least Squares (LS) direct adaptation of the forward equalizer taps over a frame interval, assuming channel time variation is slow during this interval. In this algorithm, the LS direct adaptation of the forward filter is done over several FFT blocks, which do not exceed the channel coherence time. We call this technique as Least Squares Interference Suppression (LS-IS) algorithm.

In this technique, the LS processing enhances the desired user signal by using its signatures. These signatures are the improved decisions of each ICU, provided by the updated CFR estimates obtained via a combination of the SD-IBDFE and PIC-assisted 
version of the HD-DFICE. The forward coefficients in terms of ICU channel estimates are replaced by the following LS estimates over $F$ blocks in a frame,

$$
\mathbf{W}_{k}^{j}[n]=\left[\frac{1}{F} \sum_{c=1}^{F}\left(\mathbf{X}_{k}^{j}[n, c] \mathbf{X}_{k}^{j}[n, c]^{\mathrm{H}}\right)\right]^{-1}\left[\frac{1}{F} \sum_{c=1}^{F}\left(\mathbf{X}_{k}^{j}[n, c] \tilde{S}_{k}^{j}[n, c]^{\mathrm{H}}\right)\right]
$$

where $c$ is the block index in a frame, for $c=1,2, \ldots, F$, and $\mathbf{X}_{k}^{j}[n, c]$ is the filtered array input after PIC processing obtained via (6.3) for $k$ th user after $j$ th HD-DFICE iteration for the $c$ th block in a frame and $\tilde{S}_{k}^{j}[n, c]$ represents the updated frequency domain hard decision for $k$ th user in $c$ th block at $n$th frequency after SD-IBDFE and channel decoder. $\mathbf{W}_{k}^{j}[n]$ is applied and an updated set of hard decisions for each of the ICU is obtained $\left(\tilde{S}_{k}^{j}[n]\right)$. In the next ICE iteration, the updated CFR estimates for the $k$ th ICU are computed as:

$$
\hat{\mathbf{H}}_{k}^{j}=\frac{\mathbf{X}_{k}^{j}[n]}{\widetilde{S}_{k}^{j}[n]}
$$

Afterward, a Wiener smoothing filter is used. Figure 6.9 shows a block diagram of the iterative receiver, including the LS processing to mitigate the inter-user interference.

Note that the use of LS-adapted equalizer coefficient vectors amounts to linear MMSE equalization after ICU interference removal. However the usual LE performance loss relative to IBDFE on frequency-selective channels is not significant due to the diversitycombining property of the $M$ receiving antenna elements.

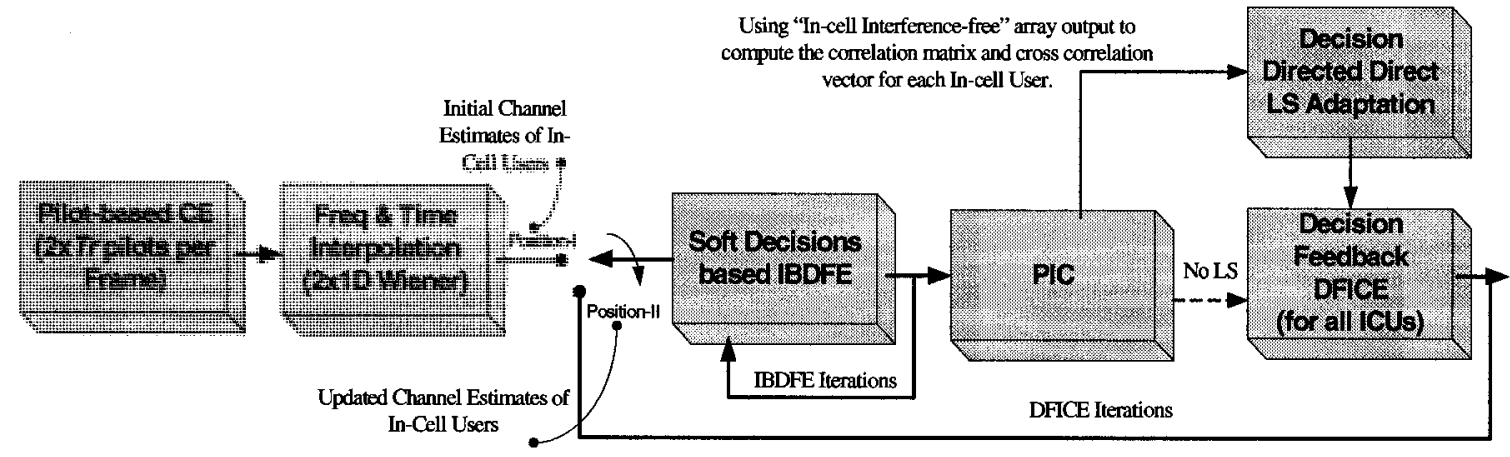

Fig. 6.9. Block diagram of iterative receiver for mitigating intra and inter-cell interference using LS forward filtering (LS-IS algorithm). 


\subsection{Simulation Results}

Figure 6.10 illustrates the LS-IS average FER performance. There are two equal power orthogonal ICUs $(K=2)$ and four OCIs each at $15 \mathrm{~dB}$ average received power below the ICU ( $P=4 @-15 \mathrm{~dB})$. Four receiving antenna elements $(M=4)$ at the BS are assumed. Following scenarios are depicted for comparison:

1. CE results with SD-IBDFE having $I_{E}=4$ using initial pilot-assisted CFR estimates (Labeled 'IBDFE').

2. LS-IS algorithm (updating of forward equalizer) with HD-DFICE results having one or two ICE iterations ( $I_{I C E}$, iter=1 and 2) (Labeled 'DFICE').

3. PCSI with SD-IBDFE having ( $I_{E}$, iter=4) (Labeled 'PCSI-IBDFE').

Two 256-symbol short training blocks per ICU are used ( $N T=2$ placed prior to the first and last symbol in a frame). Rate $1 / 2$, constraint length 7 convolutional code, QPSK modulation and independent time-varying channel on each user to antenna link with a mobility of $50 \mathrm{~km} / \mathrm{hr}$ are considered. In comparison to Figure 6.8, the improvement with the proposed LS-IS algorithm is depicted in Figure 6.10. An SNR improvement of 0.5 to $1 \mathrm{~dB}$ with two OCIs at $-15 \mathrm{~dB}$ each is achievable. It should be noted that while enhancing the signal of the desired ICU, the proposed LS approach also suppresses the intra-cell interference by about $0.8 \mathrm{~dB}$ (in the no OCI case). 


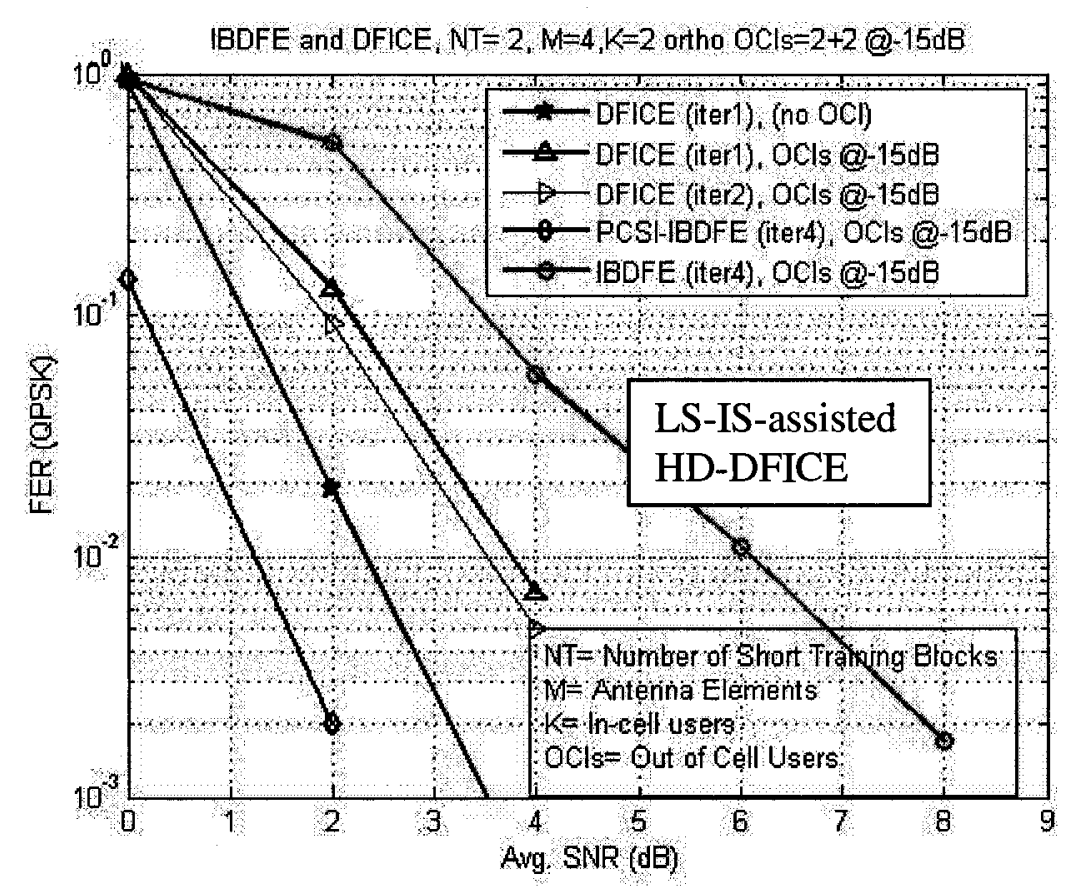

Fig. 6.10. Average FER with SD-IBDFE and LS-IS assisted HD-DFICE (uncompensated OCIs with inter and intra-cell interference), $M=4, K=2, \mathrm{C} 2-\mathrm{Channel,} 50 \mathrm{~km} / \mathrm{hr}$,

$$
N T=2, I_{\mathrm{ICE}}=1 \text { and } 2, P=4 @-15 \mathrm{~dB} \text {. }
$$

\subsection{Optimizing Pilot Overhead with LS-IS algorithm}

Figure 6.6 illustrated the performance improvement obtained incurred by a large amount of pilot overhead $(N T=K+P)$. Also in most of the simulation results presented above we use at least $N T=2$. More $N T$ costs a proportional pilot overhead. Utilizing iterative processing can reduce high pilot overhead. The idea is that more HD-DFICE iterations ( $\left.I_{\text {ICE }}\right)$ may compensate the worse initial CFR estimates caused by the lesser NT.

In this section we perform simulations keeping all the parameters the same as in above simulations other than $N T$, which is now one per user. 


\subsubsection{Simulation Scenarios}

Following equalization and $\mathrm{CE}$ algorithms are evaluated with $N T=1$ and compared with $N T=2$ (OCIs@-15dB) and shown in Figures 6.11a, 6.11b and 6.11c.

1. LE with 4 OCIs (non-iterative CE).

2. LE with no OCI (non-iterative $\mathrm{CE}$ ).

3. PIC assisted HD-DFICE with no OCI and $I_{I C E}=1$ (no LS-IS).

4. PIC assisted HD-DFICE with no OCI and $I_{I C E}=3$ (no LS-IS).

5. PIC assisted HD-DFICE with 4 OCI and $I_{I C E}=1$ (no LS-IS).

6. PIC assisted HD-DFICE with 4 OCI and $I_{I C E}=3$ (no LS-IS).

7. LS-IS based HD-DFICE with no OCI and $I_{I C E}=1$.

8. LS-IS based HD-DFICE with no OCI and $I_{I C E}=3$.

9. LS-IS based HD-DFICE with 4 OCI and $I_{I C E}=1$.

10. LS-IS based HD-DFICE with $4 \mathrm{OCI}$ and $I_{I C E}=3$. 


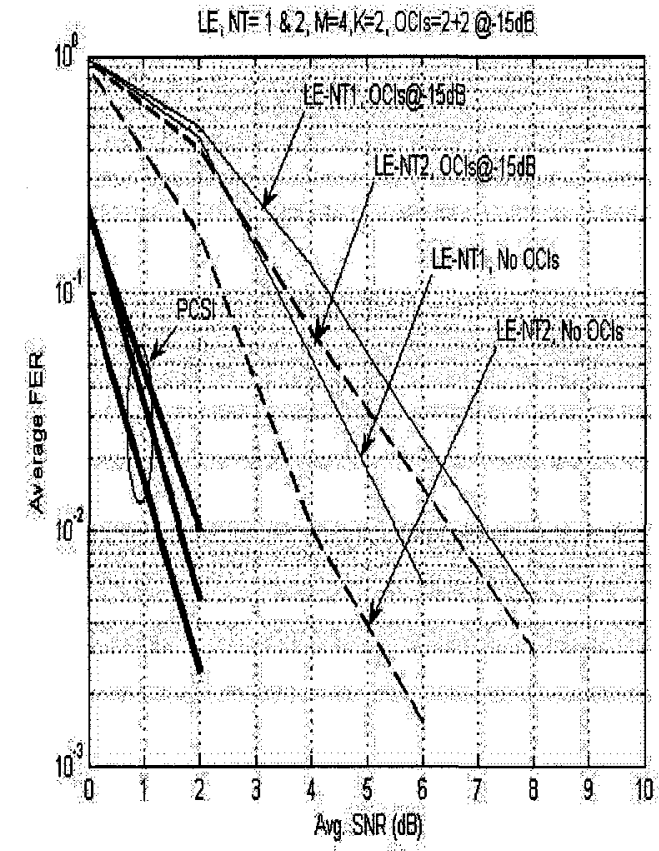

a)

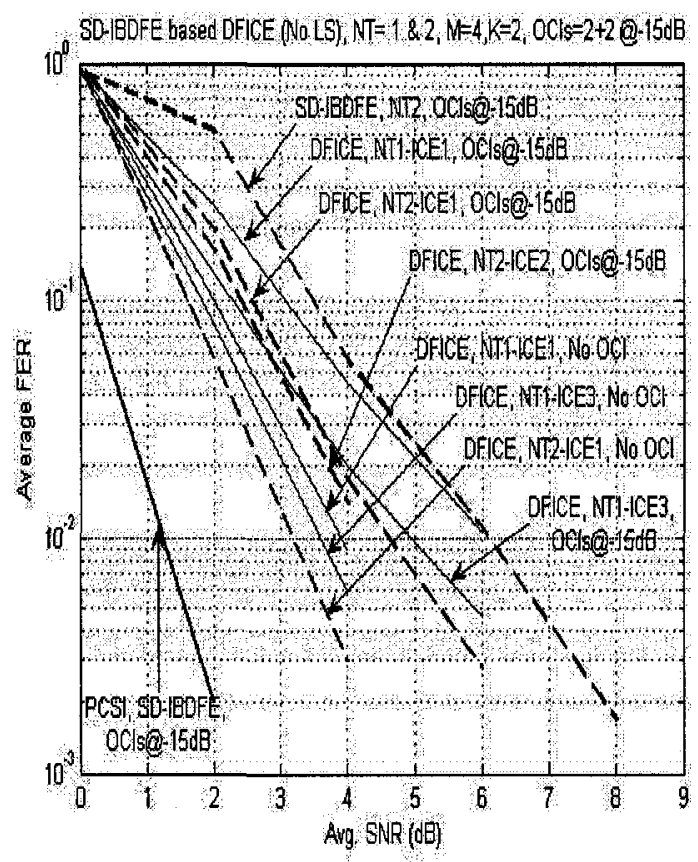

b)

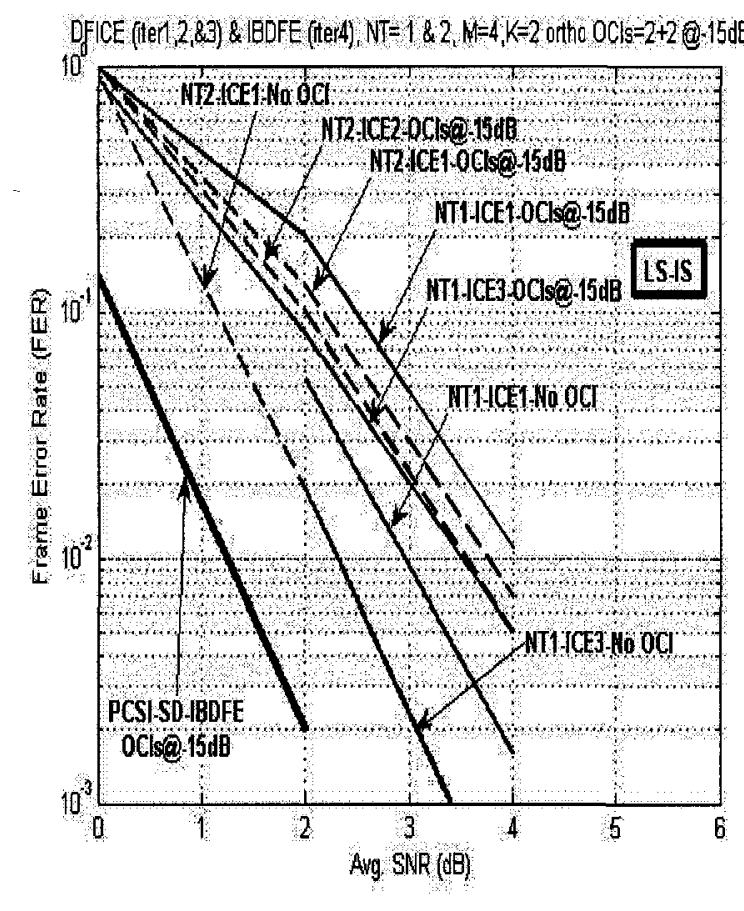

Fig. 6.11. Average FER with SD-IBDFE and LS-IS assisted HD-DFICE (uncompensated OCIs with inter and intra-cell interference), $M=4, K=2, \mathrm{C} 2-\mathrm{Channel,} 50 \mathrm{~km} / \mathrm{hr}, N T=1$ and 2, $P=4 @-15 \mathrm{~dB}$, a) LE with 0 and 4 OCIs (non-iterative CE), b) PIC assisted HD-DFICE with 0 and 4 OCI and $I_{I C E}=1$ or 3 (no LS-IS), c) LS-IS based HD-DFICE with 0 and 4 OCI and $I_{I C E}=1$ and 3 . 
We observe that by increasing $I_{I C E}$, all algorithms perform better in terms of SNR degradation. The proposed LS-IS algorithm significantly reduces SNR degradation and the average FER with $N T=1$ becomes equal to that of $N T=2$ and this is true in the presence or absence of the inter-cell interference (see Table 6.1, last column). Hence if HD-DFICE is provided with a good set of decisions (via LS-IS), the overhead penalty $(N T=2)$ can be removed.

\subsection{Overall Comparison of the Algorithms}

In this section we review the algorithms proposed and evaluated in this chapter. Table 6.1 summarizes some new results and some of the selected results from the chapter and presents a comparison in terms of SNR degradation between non-iterative and iterative CE algorithms at an average FER of $10^{-2}$ with $N T=1$ and 2 per ICU. 'All pilot transmission' results with or without inter-cell interference are also given for reference purposes. In 'All pilot transmission', we assume a transmission of a frame with all symbols known at the receiver. 
Table 6.1. Summary of the comparison between different algorithms.

\begin{tabular}{|c|c|c|c|c|}
\hline \multirow{2}{*}{$\begin{array}{l}M=4, K=2, C 2 \text {-channel } \\
50 \mathrm{~km} / \mathrm{hr} \text {., Parameters } \\
\text { and assumptions given } \\
\text { in Table } 4.1 \text { (Chapter } 4 \text { ) }\end{array}$} & \multirow{2}{*}{$\begin{array}{l}\text { SNR for } \\
\text { PCSI (dB) for } \\
10^{-2} \text { FER }\end{array}$} & \multicolumn{3}{|c|}{$\begin{array}{l}\text { SNR with CE (dB) (SNR degradation due to } \\
\text { non-perfect CSI shown in bold) }\end{array}$} \\
\hline & & $\begin{array}{l}\text { Non-iterative } \\
\text { CE (SD- } \\
\text { IBDFE) }\end{array}$ & $\begin{array}{l}\text { PIC-assisted } \\
\text { DFICE (no } \\
\text { LS forward } \\
\text { filter) }\end{array}$ & $\begin{array}{l}\text { LS-IS } \\
\text { assisted } \\
\text { DFICE }\end{array}$ \\
\hline No OCIs $(N T=1)$ & 0.3 & 4.2 (3.9) & $3.6(3.3)$ & $2.3(\mathbf{2 . 0})$ \\
\hline No OCIs $(N T=2)$ & 0.3 & $3.7(3.4)$ & $3.2(2.9)$ & $2.3(\mathbf{2 . 0})$ \\
\hline $\begin{array}{c}4 \text { OCIs, each @ }-15 \mathrm{~dB} \\
(N T=1)\end{array}$ & 1.3 & $6.3(5.0)$ & $5.0(3.7)$ & $3.5(2.2)$ \\
\hline $\begin{array}{l}4 \text { OCIs, each @ }-15 \mathrm{~dB} \\
(N T=2)\end{array}$ & 1.3 & $6.1(4.8)$ & $4.3(3.0)$ & $3.5(2.2)$ \\
\hline No OCIs $(N T=2)$ & \multicolumn{4}{|c|}{ All Pilot transmission $0.6(\mathbf{0 . 3})$} \\
\hline $\begin{array}{c}4 \text { OCIs, each @ }-15 \mathrm{~dB} \\
(N T=2)\end{array}$ & \multicolumn{4}{|c|}{ All Pilot transmission $1.5(\mathbf{0 . 2})$} \\
\hline
\end{tabular}

\section{Observations:}

- HD-DFICE performance improves with the quality of the hard decisions (See last two columns of Table 6.1.

- HD-DFICE reduces SNR degradation significantly in the presence of OCIs.

- In the presence of OCIs, doubling pilot overhead by changing $N T$ from 1 to 2 (thereby enhancing averaging over noise and OCI interference) improves initial pilot-generated CFR estimates by 0.2 to $0.7 \mathrm{~dB}$ for non-iterative and PIC-assisted HD-DFICE.

- With LS-IS algorithm the SNR degradation with $N T=1$ becomes equal to that of $N T=2$ with or without OCIs.

- The presence of 4-15 dB OCIs degrades SNR for PCSI by only $1 \mathrm{~dB}$, but degrades SNR with CE by several dBs.

- All pilot transmission results dictate that a further improvement room with the improvement of the decisions via better CE is still present. This could be achieved 
by using soft decision in DFICE with turbo processing. The Soft Decision-based DFICE (SD-DFICE) is addressed in Chapter 7.

\section{SNR degradation due to the following reasons:}

- HD-DFICE is sensitive to multiple user fed back decision errors.

- The sensitivity of the equalizer output to errors in multiple interferers' channels.

- Uncompensated out of cell interference to data (unless LS-IS is used).

- HD-DFICE combined with PIC in the presence of OCIs is sensitive to inaccurate subtraction of ICU interference due to the $\mathrm{CE}$ and hard decision errors.

- At the low SNR levels in the simulations, initial and iterative CFR estimates are noisy.

\subsection{Channel Estimation over Multiple Frames}

The time averaging is involved in the initial pilot-based $\mathrm{CE}$ as well as in the proposed LS-IS algorithm to suppress the inter-user interference. The performance of such schemes could further be improved at slight complexity increase if the time averaging is performed over larger number of SC-FDE blocks or in general blocks per frame or over multiple frames. From initial pilot-based CE point of view, the interpolation and smoothing of channel estimates over more than one frame also has the potential of improving the performance, as long as the number of frames does not exceed the user channels' coherence times. We denote the total number of consecutive frames (by considering a full duplex communication system) as $F$ r. $F r=1$ means averaging over one frame and $F \mathrm{r}=2$ means averaging over two consecutive frames, while each frame consists of 12 blocks. Figure 9.1 shows a scenario with $F_{\mathrm{r}}=2$. 


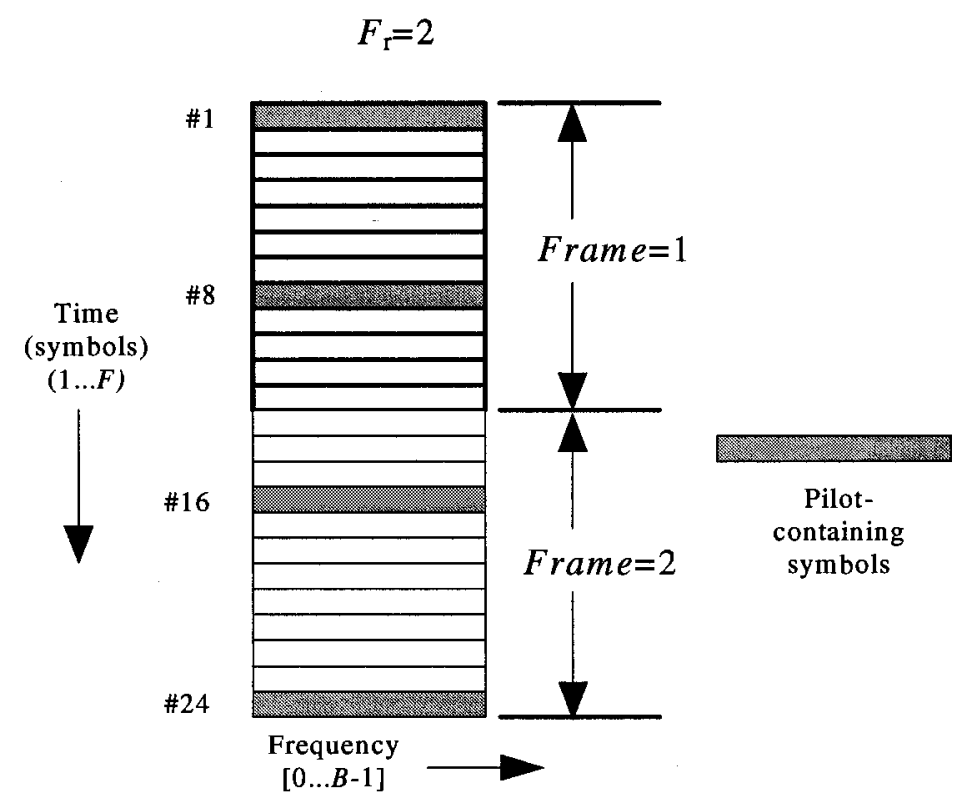

Fig. 6.12. Multi-frame (full-duplex) $\mathrm{CE}$ structure $\left(F_{\mathrm{r}}=2\right)$.

Block number 1, 8, 16 and 24 are the pilot carrying blocks. There is no difference in the frequency axis interpolation, while in time direction linear interpolation is carried out between the blocks. Each frame consists of one code block.

It is illustrated in Figure 6.13 that an improvement of about $0.5 \mathrm{~dB}$ is obtained by exploiting the multi-frame $C E$ technique with $F_{\mathrm{\Gamma}}=2, M=4, K=2$, C2-Channel, $50 \mathrm{~km} / \mathrm{hr}$, $N T=2, P=4 @-15 \mathrm{~dB}$ and $I_{I C E}=2$. SNR degradation with $F_{\mathrm{r}}=1$ and $F_{\mathrm{r}}=2$ is compared in Table 6.2. Extra frames are effective at slow vehicular speeds. At higher speeds CE over several frames might not perform better than one frame case. 


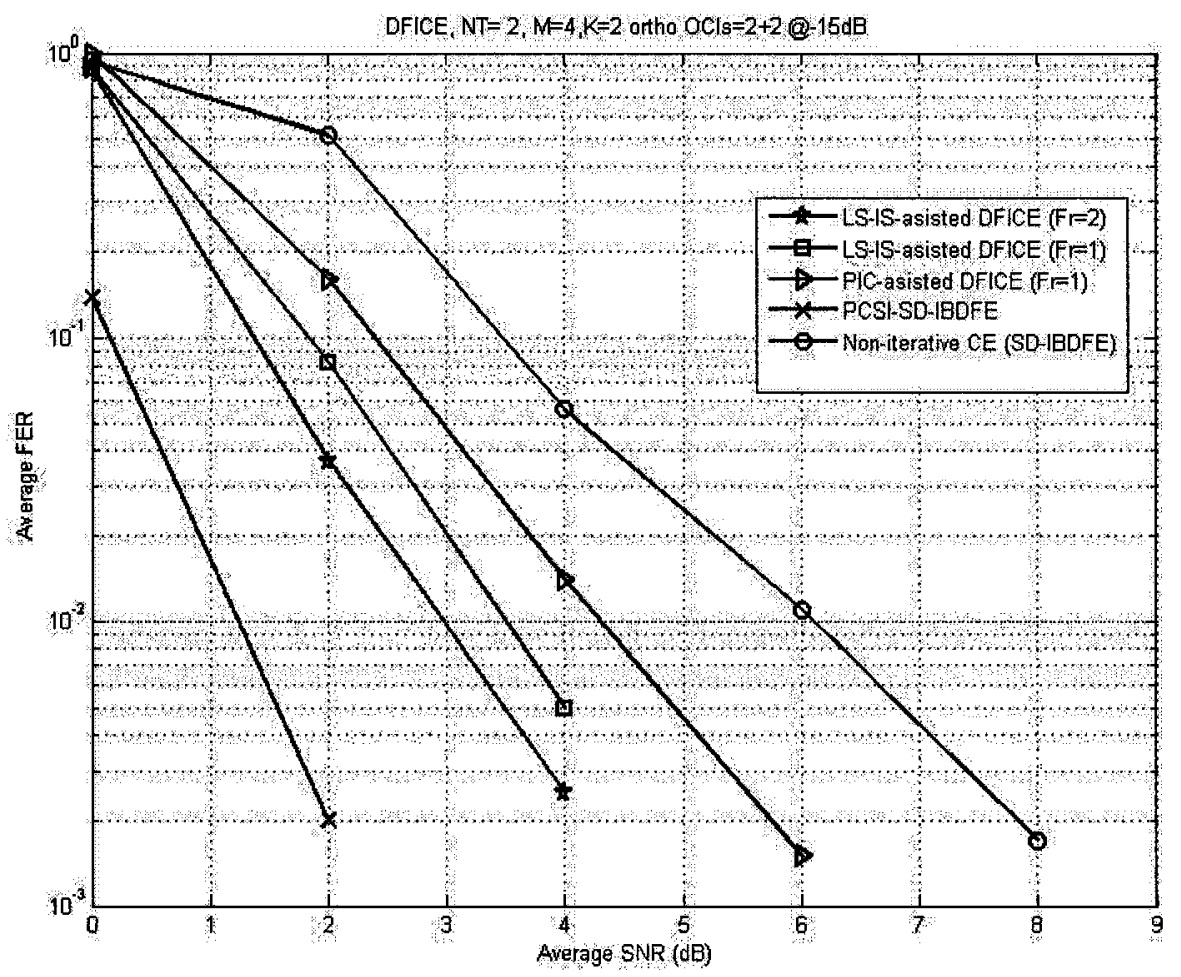

Fig. 6.13. Average FER comparison with $F_{\mathrm{r}}=1$ or 2 , non-iterative CE SD-IBDFE and LSIS assisted HD-DFICE , $M=4, K=2, I_{I C E}=2$, C2-Channel, $50 \mathrm{~km} / \mathrm{hr}, N T=2, P=4 @-15 \mathrm{~dB}$.

Table 6.2. Improvement via CE over multiple frames (LS-IS).

\begin{tabular}{|c|c|c|c|c|}
\hline \multirow{2}{*}{$\begin{array}{l}M=4, K=2, \text { C2-channel } \\
50 \mathrm{~km} / \mathrm{hr} ., \text { Parameters } \\
\text { and assumptions given } \\
\text { in Table } 4.1 \text { (Chapter 4) }\end{array}$} & \multirow{2}{*}{$\begin{array}{c}\text { SNR for } \\
\text { PCSI (dB) for } \\
10^{-2} \text { FER }\end{array}$} & \multicolumn{3}{|c|}{$\begin{array}{l}\text { SNR with CE (dB) (SNR degradation due to } \\
\text { non-perfect CSI shown in bold) }\end{array}$} \\
\hline & & $\begin{array}{l}\text { Non-itera tive } \\
\text { CE (SD- } \\
\text { IBDFE) }\end{array}$ & $\begin{array}{l}\text { PIC-assisted } \\
\text { DFICE (no } \\
\text { LS forward } \\
\text { filter) }\end{array}$ & $\begin{array}{l}\text { LS-IS } \\
\text { assisted } \\
\text { DFICE }\end{array}$ \\
\hline $\begin{array}{l}4 \text { OCIs, each @ }-15 \mathrm{~dB} \\
(N T=2), F \mathrm{r}=1\end{array}$ & 1.3 & $6.1(4.8)$ & $4.3(3.0)$ & $3.5(2.2)$ \\
\hline $\begin{array}{l}4 \text { OCIs, each @ }-15 \mathrm{~dB} \\
(N T=2), F \mathrm{r}=2\end{array}$ & \multicolumn{4}{|c|}{$\begin{array}{c}\text { CE over multiple frames (LS-IS assisted DFICE) } \\
3.0(\mathbf{1 . 7})\end{array}$} \\
\hline
\end{tabular}

\subsection{Summary}

In this chapter, we developed iterative receiver processing techniques for the improvement of the desired ICUs' channel estimates in the presence of intra-cell and 
inter-cell interference. To deal with intra-cell interference, PIC is used in coordination with the SD-IBDFE. Moreover, a new PIC-assisted hard decision-based DFICE receiver structure is introduced, which utilizes the LS adaptation to feed the DFICE with decisions having lesser impact of the OCIs. It is also shown that HD-DFICE based on better decisions provided by the LS-IS can improve the channel estimates in the presence of inter-user interference at lower pilot overheads. Also, iterative CE over multiple frames gives some additional SNR gain. 


\section{Chapter 7}

\section{Turbo Equalization based Iterative Channel Estimation}

\subsection{Introduction}

In this chapter, we modify the threshold-based HD-DFICE technique by replacing hard decisions with the soft estimates of the symbols derived from a Low-Density ParityCheck (LDPC) code-based channel decoder. In Soft Decision-based DFICE (SD-DFICE) the extrinsic information is used in the soft mapping of the symbols. An Expectation Maximization (EM) algorithm is used to derive the SD-DFICE algorithm, which also provides a rationale to justify the use of FRT algorithm presented in Chapter 6. The multi-user frequency domain adaptive soft interference cancellation detector SD-IBDFE is modified to work with the turbo processing. The effect of OCIs is suppressed by using a Sub-Space (SS) technique in which the correlation matrices of the OCIs are estimated. An average FER comparison between the hard and soft decision-based DFICE is presented. Finally, a brief computational complexity analysis of the DFICE and interference suppression algorithms is presented.

\subsection{Overview of Frequency Domain Turbo Equalization}

Separating the channel equalization (SD-IBDFE) and decoding is a sub-optimal way of equalizing the channel. Turbo Equalization (TE) is an iterative receiver processing in which equalization and decoding tasks are repeated on the same set of received vectors. The concept of turbo decoding can be merged with the equalization process in order to improve the equalization performance with the aid of soft outputs provided by an equalizer. A turbo decoder consists of two Soft-In Soft-Out (SoISoO) decoders creating two dimensional (2-D) memory. In case of the TE, the 2-D memory is provided by the 
channel encoder at the transmitter and the ISI caused by the multi-path Rayleigh fading channel. The soft information generated by either equalizer or the decoder is used as $a$ priori information by the other component. TE achieves reliable communication over ISI channels and shows low BER at low and medium SNRs with iterative decoding. [TKS02] and references therein describe a theoretical and historical background on frequency domain TE. With TE in SISO link, [TKS02] shows a BER performance improvement. An iterative time domain soft ISI cancellation is presented in [ATM03] utilizing the TE in MIMO channels, in the presence of unknown co-channel users. [ATM03] estimates the correlation matrices of the unknown users by time averaging over several OFDM blocks. [RW02] proposes a blind suppression of the unknown Multiple Access Interference (MAI) in synchronous and asynchronous CDMA systems with TE. A SC-FDE system is considered in [KHS03], with the inclusion of a so-called turbo-like algorithm to improve the BER performance. In that work, DFE is implemented in the time domain with soft decision only taken in the first feedback step. A comparison with turbo processing between MIMO OFDM and MIMO SC-FDE with coded and spatially multiplexed data is presented in [YSSO4]. It is an extension of a SISO TE approach described in [TSK02]. [SYS03] describes the CE aspects, by using RLS adaptation-based TE in MIMO-OFDM. Recent work on frequency domain TE, with SC-FDE signals is presented in [NLF07]. In that work, different equalization and CE approaches with turbo processing are evaluated and compared.

\subsubsection{Low-Density Parity-Check (LDPC) code-based Turbo}

\section{Equalization}

A frequency domain TE comparison between Low-Density Parity-Check (LDPC) codebased TE and the convolutional code-based TE is given in [SF06]. There is an error floor at higher SNRs with the convolutional code-based TE and this motivates the use of LDPC code-based TE. Therefore, in a TE-based receiver, we will be using Low-Density Parity-Check (LDPC) codes for TE. LDPC codes are recently re-discovered error correcting linear block codes [Gal62]. LDPC code can be fully described by the associated sparse parity-check matrix. The decoding process is done iteratively. If the 
number of iterations is too small, the algorithm may not converge. The LDPC coder/decoder does not require any interleaver/de-interleaver, which reduces the complexity of the LDPC code-based TE. Unlike other codes, a LDPC decoder cannot be directly connected to the output of an LDPC encoder, as the decoder requires LogLikelihood Ratios (LLRs). Therefore, a demodulator or a SoISoO equalizer (described in Section 7.3) is needed with LDPC decoder. [KFS06] describes the MIMO OFDM CE aspects with TE using LDPC coding.

\subsection{Soft-In Soft-Out IBDFE-based TE}

The receiver comprises a modified version of SD-IBDFE presented in Chapter 5 named Soft-In Soft-Out (SoISoO) IBDFE. In SoISoO-IBDFE the SD-IBDFE is altered and improved to fit the requirements of the turbo processing. SoISoO-IBDFE generates Log Likelihood Ratio(s) (LLR(s)) to be fed into the LDPC decoder and also uses a-priori LLRs from the LDPC decoder in iterations $i>1$. The SoISoO-IBDFE generates LLRs for each of the code bits for the $k$ th desired ICU. It should be noted that receiver processing including frequency domain TE is performed for the detection and CFR estimation of the ICUs' only since the OCIs may be at low power levels.

Let the $k$ th ICU's transmitted QPSK symbol $s^{k}(q)=a^{k}(q)+j b^{k}(q)$. By exploiting the concept of LLRs for the Binary Phase Shift Keying (BPSK) transmission [ATM03] and [TKS02], the SoISoO-IBDFE computes the a-posteriori LLRs $\vec{\Omega}_{e}\left[a^{k}(q)\right]$ of the real part of the $k$ th ICU's transmitted symbol, 1 and 0 as:

$$
\vec{\Omega}_{e}\left[a^{k}(q)\right]=\ln \left(\frac{P\left[a^{k}(q)=1 \mid \mathbf{y}(q)\right]}{P\left[a^{k}(q)=0 \mid \mathbf{y}(q)\right]}\right)
$$

where $k=1,2, \ldots, K$ is the ICU index. The magnitude of $\vec{\Omega}_{c}\left[a^{k}(q)\right]$ drives the reliability of the information about the specific bit. With the help of Bayes' rule (7.1) can be written as:

$$
\vec{\Omega}_{c}\left[a^{k}(q)\right]=\ln \left(\frac{P\left[\mathbf{y}(q) \mid a^{k}(q)=1\right]}{P\left[\mathbf{y}(q) \mid a^{k}(q)=0\right]}\right)+\ln \left(\frac{P\left[a^{k}(q)=1\right]}{P\left[a^{k}(q)=0\right]}\right)
$$


The first part of (7.2) is the current extrinsic information based on the received vector $\mathbf{y}(q)$, and the second part is the a-priori LLR of the code bit $a^{k}(q)$ from the previous iteration of the decoder. As shown in Figure 7.1, (7.2) can be written as:

$$
\vec{\Omega}_{e}\left[a^{k}(q)\right]=\ddot{\Delta}_{1}\left[a^{k}(q)\right]+\ddot{\Delta}_{2}^{p r u t}\left[a^{k}(q)\right]
$$

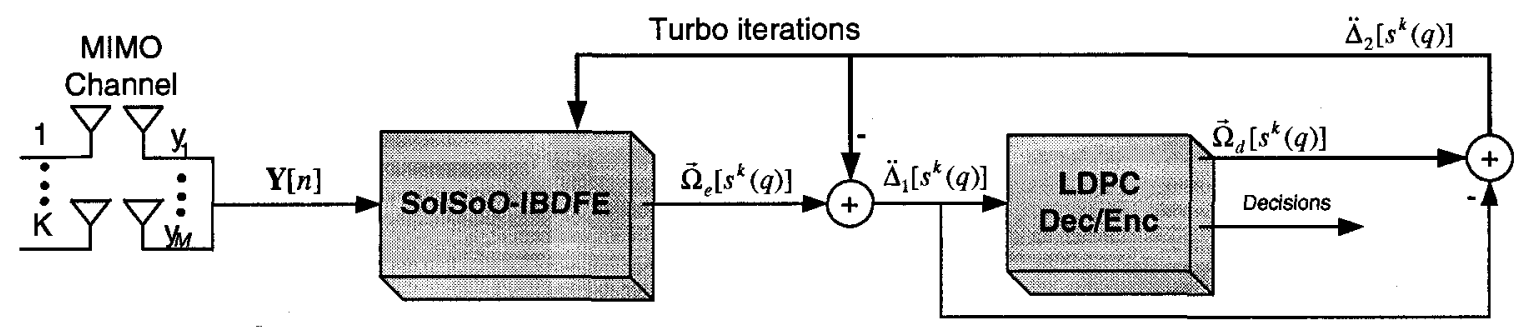

Fig. 7.1. Simplified Block diagram of LDPC code-based TE.

$\ddot{\Delta}_{1}\left[a^{k}(q)\right]\left(1^{\text {st }}\right.$ part of (7.3)) denotes the extrinsic information regarding the $k$ th ICU generated by the SoISoO-IBDFE and is fed to the LDPC decoder. $\ddot{\Delta}_{2}^{p r v}\left[a^{k}(q)\right]$ (2nd part of (7.3)) is the a-priori information at the output of the decoder to be fed to the SoISoOIBDFE in the iterative processing. The superscript " "prev" stands for the previous iteration. By approximating the equalizer output as a Gaussian random variable, the extrinsic information fed to the decoder can be derived as [RW02]:

$$
\ddot{\Delta}_{1}\left[a^{k}(q)\right]=\ln \left(\frac{P\left[\mathbf{y}(q) \mid a^{k}(q)=1\right]}{P\left[\mathbf{y}(q) \mid a^{k}(q)=0\right]}\right)
$$

$\ddot{\Delta}_{1}\left[a^{k}(q)\right]$ can be computed by finding the mean and variance at the equalizer output after each SoISoO-IBDFE iteration. Soft interference cancellation is done in the SoISoOIBDFE as described in Chapter 5. The SoISoO LDPC decoder computes the a-posteriori LLR using the prior information for each coded bit $(q=0,1, \ldots B-1)$ given by:

$$
\vec{\Omega}_{d}\left[a^{k}(q)\right]=\ln \left(\frac{P\left[a^{k}(q)=1 \mid \ddot{\Delta}_{1}^{p r v}\left[a^{k}(q)\right]\right.}{P\left[a^{k}(q)=0 \mid \ddot{\Delta}_{1}^{p r v}\left[a^{k}(q)\right]\right.}\right)
$$

or

$$
\vec{\Omega}_{d}\left[a^{k}(q)\right]=\ddot{\Delta}_{2}\left[a^{k}(q)\right]+\ddot{\Delta}_{1}^{p r v}\left[a^{k}(q)\right]
$$


In (7.6), $\ddot{\Delta}_{2}\left[a^{k}(q)\right]$ is the extrinsic information fed back to the SoISoO-IBDFE and $\ddot{\Delta}_{1}^{p r v}\left[a^{k}(q)\right]$ is the a-priori information fed to the decoder from the equalizer. In a similar way LLR for the imaginary part $b^{k}(q)$ is computed. The LDPC decoder also computes $a$ posteriori LLR for every information bit so that the final hard decision can be made after the final iteration. These hard decisions are comparatively better than the ones with the non-turbo receivers, at the cost of the complexity associated with the TE.

For each ICU the soft feedback information derived from the channel decoder output can be exploited in order to derive the estimates of transmitted data symbols. We here focus at first on the computation of the data symbol estimates for each ICU. The soft information in the form of LLRs is converted into symbol estimates through soft mapping [LGL01]. Eventually the ICE techniques presented later in the chapter make use of hard decisions or the data symbol estimates derived from the output of the channel decoder in addition to the pilot symbols. Finally, these updated estimated CFRs are fed into the proposed TE assisted LS-IS and SS-IS algorithms.

The objective is to derive the estimated value of the $k$ th ICU's transmitted QPSK symbol $\hat{s}^{k}(q)=\hat{a}^{k}(q)+j \hat{b}^{k}(q)$ from coded data LLRs provided by the channel decoder. The estimated $\hat{a}^{k}(q)$ is approximated by its mean value [LGL01] given by:

$$
\hat{a}^{k}(q)=E\left(\hat{a}^{k}(q) \mid \ddot{\Delta}_{1}\left[a^{k}(q)\right]\right)
$$

The $\hat{a}^{k}(q)$ can be expressed by:

$$
\hat{a}^{k}(q)=E\left[\hat{a}^{k}(q) \mid \ddot{\Delta}_{1}\left[a^{k}(q)\right]=P\left\{\hat{a}^{k}(q)=1 \mid \ddot{\Delta}_{1}\left[a^{k}(q)\right\}-P\left\{\hat{a}^{k}(q)=0 \mid \ddot{\Delta}_{1}\left[a^{k}(q)\right\}\right.\right.\right.
$$

The extrinsic information of the independent and independent and identically distributed coded data can be written as:

$$
\ddot{\Delta}_{2}\left[a^{k}(q)\right]=\ln \left(\frac{P\left[a^{k}(q)=1 \mid \ddot{\Delta}_{1}\left[a^{k}(q)\right]\right.}{1-P\left[a^{k}(q)=1 \mid \ddot{\Delta}_{1}\left[a^{k}(q)\right]\right.}\right)
$$

The probability of having $a^{k}(q)=1$ is given by:

$$
P\left[a^{k}(q)=1 \mid \ddot{\Delta}_{1}\left[a^{k}(q)\right]=\frac{\exp \left(\ddot{\Delta}_{2}\left[a^{k}(q)\right]\right)}{1+\exp \left(\ddot{\Delta}_{2}\left[a^{k}(q)\right]\right)}\right.
$$

Similarly, 
$P\left[a^{k}(q)=0 \mid \ddot{\Delta}_{1}\left[a^{k}(q)\right]=1-P\left[a^{k}(q)=1 \mid \ddot{\Delta}_{1}\left[a^{k}(q)\right]=\frac{1}{1+\exp \left(\ddot{\Delta}_{2}\left[a^{k}(q)\right]\right)}\right.\right.$

(7.8) becomes,

$$
\begin{aligned}
\hat{a}^{k}(q)= & P\left\{a^{k}(q)=1 \mid \ddot{\Delta}_{1}\left[a^{k}(q)\right\}-P\left\{a^{k}(q)=0 \mid \ddot{\Delta}_{1}\left[a^{k}(q)\right\}\right.\right. \\
& =\frac{\exp \left(\ddot{\Delta}_{2}\left[a^{k}(q)\right]\right)}{1+\exp \left(\ddot{\Delta}_{2}\left[a^{k}(q)\right]\right)}-\frac{1}{1+\exp \left(\ddot{\Delta}_{2}\left[a^{k}(q)\right]\right)} \\
& =\frac{\exp \left(\ddot{\Delta}_{2}\left[a^{k}(q)\right]\right)-1}{\exp \left(\ddot{\Delta}_{2}\left[a^{k}(q)\right]\right)+1}=\tanh \left(\frac{\ddot{\Delta}_{2}\left[a^{k}(q)\right]}{2}\right)
\end{aligned}
$$

where $\tanh (x)$ represents the tangent hyperbolic function of $x$. Similarly soft value for the imaginary part $\hat{b}^{k}(q)$ can be found.

The above proposes a solution to convert the binary decoder output into a soft estimate of the symbol. Hence the modification in the SD-IBDFE in order to make it SoISoO-IBDFE is a slight change in the soft decision computation (5.10). The soft decisions will now be computed using the a-priori LLRs, instead of the conditional MSE from the equalizer's output.

\subsection{TE with ICE and Interference Suppression}

In this section, we apply the threshold-based HD-DFICE presented in Chapter 6 in a TEbased receiver. The TE-based HD-DFICE performance is expected to improve because of the soft information transfer between SoISoO-IBDFE and the decoder. The exchange of extrinsic information between SoISoO-IBDFE and the LDPC decoder is repeated iteratively and helps to improve the final hard decisions. Channel estimates of the cochannel ICUs with these decisions are used to cancel the intra-cell interference with PIC processing in a similar way as described in Chapter 6. The LS-IS desired signal enhancement algorithm further reduces the effects of the OCIs. Decisions obtained after PIC and LS-IS algorithm are used in the HD-DFICE. Also a Sub-Space (SS) approach is used to further suppress the effects of the OCIs at the array output [RW02]. We name this Sub-Space Interference Suppression (SS-IS) approach. The process of TE-assisted HDDFICE is repeated until reliable estimates are obtained. 
Moreover, we introduce a Soft Decision-based DFICE (SD-DFICE) in the SC-FDE multi-user system. It is shown in [Win04] that in a SISO-OFDM system the a-posteriori probability-based SD-DFICE gives better performance in the mean squared error (MSE) sense compared to the extrinsic information-based SD-DFICE. But there is not much difference in the average FER results. In this work we will be using extrinsic information in the SD-DFICE algorithm. Later in this chapter we show that soft decision based DFICE exhibits better error propagation performance and outperforms the hard decisionsbased DFICE in an interference-prone SC-FDE system. A simplified implementation block diagram of DFICE in general is shown in Figure 7.2.

It is mentioned in Chapter 6 that in SC-FDE, the envelope of the frequency response of the decisions is not constant, which creates noise enhancement at certain frequencies while computing the CFR via decisions. A threshold based frequency replacement solution named FRT algorithm was introduced in which the CFR obtained from decisions at noise enhancement frequencies are replaced by the CFR estimates of the previous ICE iteration. While deriving the SD-DFICE via an Expectation Maximization (EM) algorithm we also provide a rationale to justify the use of FRT algorithm. It addresses and solves the problem of noise enhancement in SC-FDE HD-DFICE and SD-DFICE channel estimates due to data sequence frequency selectivity.

\subsection{Frequency Domain TE-based HD-DFICE}

The performance of the decision-directed iterative processing heavily depends on the quality of the decisions. Especially in an interference prone system the decisions are not only affected by the noise but also by the inter-user interference. LS-IS proposed in Chapter 6 uses PIC to filter out the effects of ICUs with the help of the decisions made in the preceding iteration. Similarly the LS processing which was included to remove the effects of the OCIs also utilizes the decisions of the desired user. Hence the quality of the decisions influences the performance of the LS-IS algorithm. Here we attempt to improve the decision-quality and eventually the performance of the LS-IS algorithm by using TE. In the TE based receiver, a hard symbol decision can be made by decoding the equalizer output to obtain the decisions on the information bits and then re-encode to get the coded bits for symbol mapping. Then these hard decisions are used in HD-DFICE. With HD- 
DFICE, error propagation may occur in the iterations when wrong decisions are made. Even if decisions are correct, undesirable noise enhancement occurs at frequencies where $\tilde{S}_{k}^{j}[n]$ is small. On the other hand, using soft decisions in which the estimator is derived from an EM principle [LWL07] can reduce error propagation and improve the DFICE performance (as given in Section 7.8).

A brief description of the LS-IS assisted HD-DFICE with TE is summarized as:

1. Get the initial pilot-aided CFR estimates for all the ICUs (Using orthogonal TMT or FMT pilots).

2. Perform the $2 \times 1 \mathrm{D}$-Wiener filter interpolation to estimate channels at non-pilot frequencies and times.

3. Use the SoISoO-IBDFE to separate the ICUs and obtain the hard decisions $\tilde{s}^{k}(i)$ for each ICU with LDPC decoding, while ignoring the OCIs.

4. Using PIC, parallel cancellation of the in-cell interference is performed using incell hard decisions.

5. LS adaptation of SDMA equalization for residual signals to suppress the OCIs.

6. Improving the channel estimates with HD-DFICE and repetition of the above steps.

\subsubsection{TE-Modified WINNER Frame Structure, Simulation}

\section{Assumptions and System Parameters}

The LDPC code-based TE is applied to a system with intra and inter-cell interference. The FFT size is 1152 data symbols $(B=1152)$. The signal is generated in the frequency domain by a larger IFFT (2048), so as to be over-sampled. The symbol rate is 45 Msymbols/s. Each transmitted signal is QPSK with rate $1 / 2$ LDPC coding. Two ICUs are considered $(K=2)$. The average received powers of the ICUs, which transmit simultaneously in a common bandwidth, are assumed equal as a result of the application of slow-acting power control. As well, there are 4 OCIs $(P=4)$ transmitting to other BSs. The average received power of the OCIs is significantly below that of the ICUs (at - $15 \mathrm{~dB}$ and at $-18.2 \mathrm{~dB}$ ). This assumption is consistent with the use of a frequency reuse partitioning or dynamic channel assignment strategy to reduce average OCI power. Each 
transmission path is modeled as an independent complex Gaussian random variable with Jakes model Doppler variations. Each transmitted signal arrives at each receiving antenna through an independent $\mathrm{C} 2$ channel with a vehicle speed of $50 \mathrm{~km} / \mathrm{hr}$. An assumed carrier frequency of $3.7 \mathrm{GHz}$ yields a maximum Doppler frequency of about $170 \mathrm{~Hz}$. The average SNR is signal to noise ratio per receive antenna. It does not include OCI power. The simulations include any error propagation effects resulting from feedback of incorrect hard decisions.

All users' 12-block frames $(F=12)$ are assumed to be perfectly synchronized. To the first and twelfth block ( $F=1$ or 12) of each frame is added $N T$ short training blocks per in-cell user, containing a Chu sequence of $T_{\mathrm{r}}$ pilot symbols. The resulting pilot waveform is therefore uniform in amplitude and spectrum. All in-cell pilots are orthogonal and do not interfere with one another. The interference distribution is a load-sharing situation when one half of the OCIs' training overlaps one of the ICU and the other half of the OCIs overlaps the other ICU. The frame size is not equal to the code block size, rather three code blocks constitute a frame. In simulation results, inside each ICE iteration $\left(I_{\mathrm{ICE}}\right)$, the receiver performs several (typically 4) turbo equalization/decoding iteration $\left(I_{\mathrm{E}}\right)$. Average power delay profile of the C2 Urban Macro multi-path time-varying channel is given in Table 2.1. The simulation parameters are summarized in Table 7.1. 
Table 7.1. WINNER Frame structure, simulation assumptions and system parameters.

\begin{tabular}{|c|c|}
\hline $\begin{array}{c}\text { Number of sub-carriers in a block or } \\
\text { FFT size }(X)\end{array}$ & 2048 \\
\hline Modulation Scheme & QPSK \\
\hline Carrier frequency & $3.7 \mathrm{GHz}$ \\
\hline Signal BW & $45 \mathrm{MHz}$ \\
\hline Sub-Carrier spacing & $39.0625 \mathrm{KHz}$ \\
\hline Number of blocks per frame & 12 \\
\hline Used sub-carriers $(B)$ & $1152(45 \mathrm{MHz} / 39.0625 \mathrm{KHz})$ \\
\hline $\begin{array}{l}\text { Short training blocks for time averaging } \\
(N T) \text { per user }\end{array}$ & 1 or 2 \\
\hline $\begin{array}{c}\text { Number of pilot symbols per training } \\
\text { block }\left(T_{r}\right)\end{array}$ & $T_{r}=384$ or 288 \\
\hline Number of pilots/frame & $T_{r}^{*} N T$ \\
\hline Number of blocks/Frame & $F=12$ \\
\hline Number of In-Cell users & $K=2$ (Orthogonal training) \\
\hline Number of receiving elements & $M=3$ \\
\hline Number of OCIs & $2+2$ \\
\hline Code Block Length & 4608 \\
\hline Turbo Iterations $\left(I_{\mathrm{E}}\right)$ & 4 \\
\hline DFICE Iterations $\left(I_{\mathrm{ICE}}\right)$ & 1 or 2 \\
\hline Reliability Threshold in DFICE $(\lambda)$ & 13 \\
\hline LDPC Decoder Iteration & 6 \\
\hline LDPC Decoder Type & $\begin{array}{l}\text { rate- } 1 / 2 \text {, regular(3,6), Sparse parity } \\
\text { check matrix dimension }=2304 \times 4608\end{array}$ \\
\hline
\end{tabular}




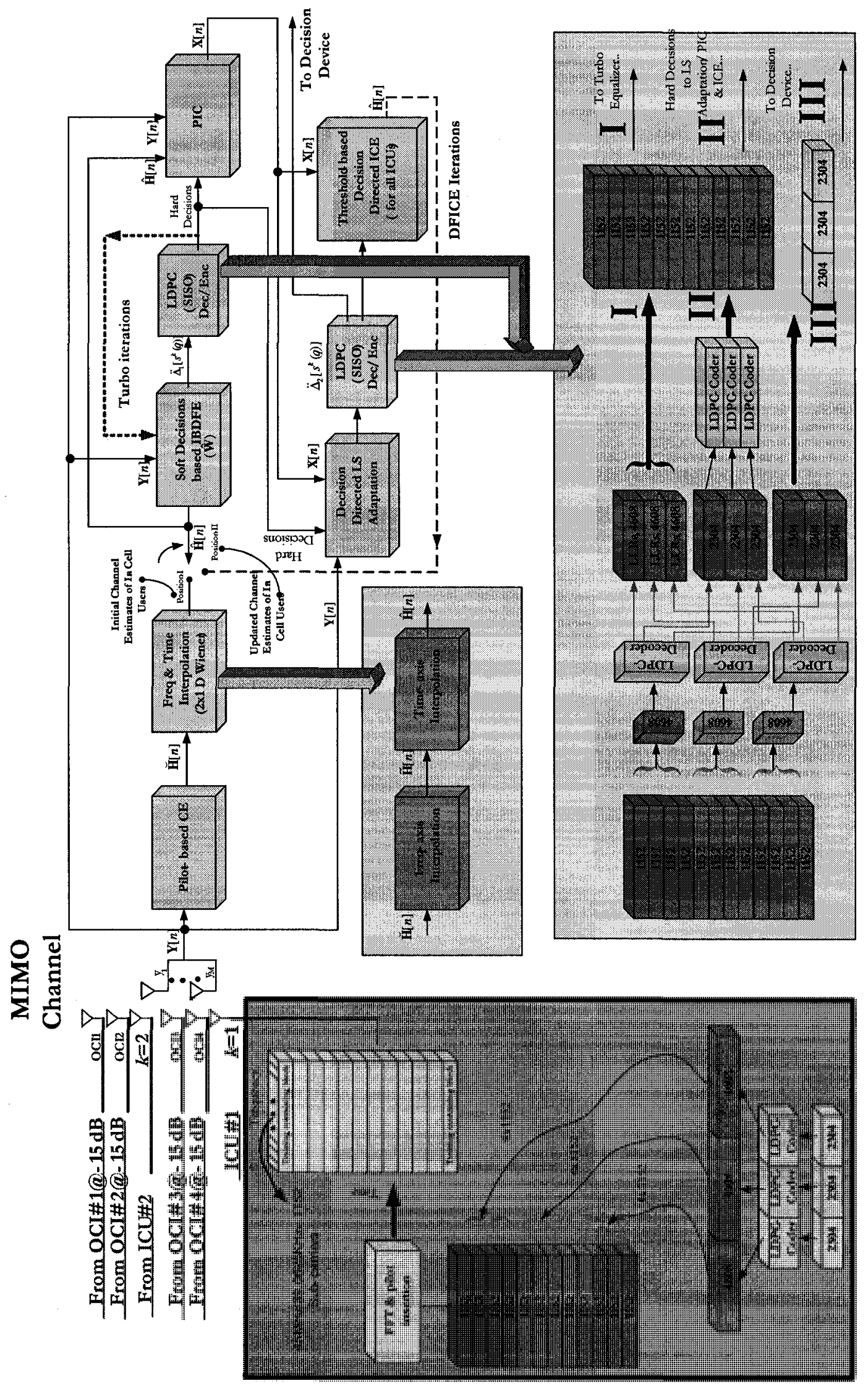

䓪 กิ่ 


\subsubsection{Simulation Results}

In this section, we evaluate the average FER performance of the proposed LS-IS algorithm with LDPC code-based TE. Figure 7.3 shows average FER comparison between simple SoISoO-IBDFE with four iterations $\left(I_{\mathrm{E}}=4\right)$ (Labeled 'only IBDFE') and the LS-IS algorithm with TE (Labeled 'LS'), with and without HD-DFICE. There are four OCIs and two ICUs in the system. For OCIs, an average received power level of $15 \mathrm{~dB}$ below the ICUs are considered. The varying parameter in LS-IS with TE is the number of short training blocks per user, which directly affects the quality of the initial channel estimates. As with TE, we work at lower levels of SNRs therefore, reliable initial estimates are crucial. Initial channel estimates are improved by sending more short training blocks while the channel estimates are further improved with the HD-DFICE due to the proposed LS-IS adaptation. An improvement of around $0.5 \mathrm{~dB}$ is achieved with two short training blocks compared to the single block. Three BS antenna elements and one HD-DFICE iteration $\left(I_{\mathrm{ICE}}=1\right)$ is considered. It should be noted that $I_{\mathrm{ICE}}$ iterations are the outer iterations and include the inner $I_{\mathrm{E}}$ iterations. PCSI with SoISoO-IBDF TE curve, is also presented for reference purposes. The "Only IBDFE" uses pilot-based estimated CFR utilizing the $2 \times 1 \mathrm{D}$ Wiener interpolation. 


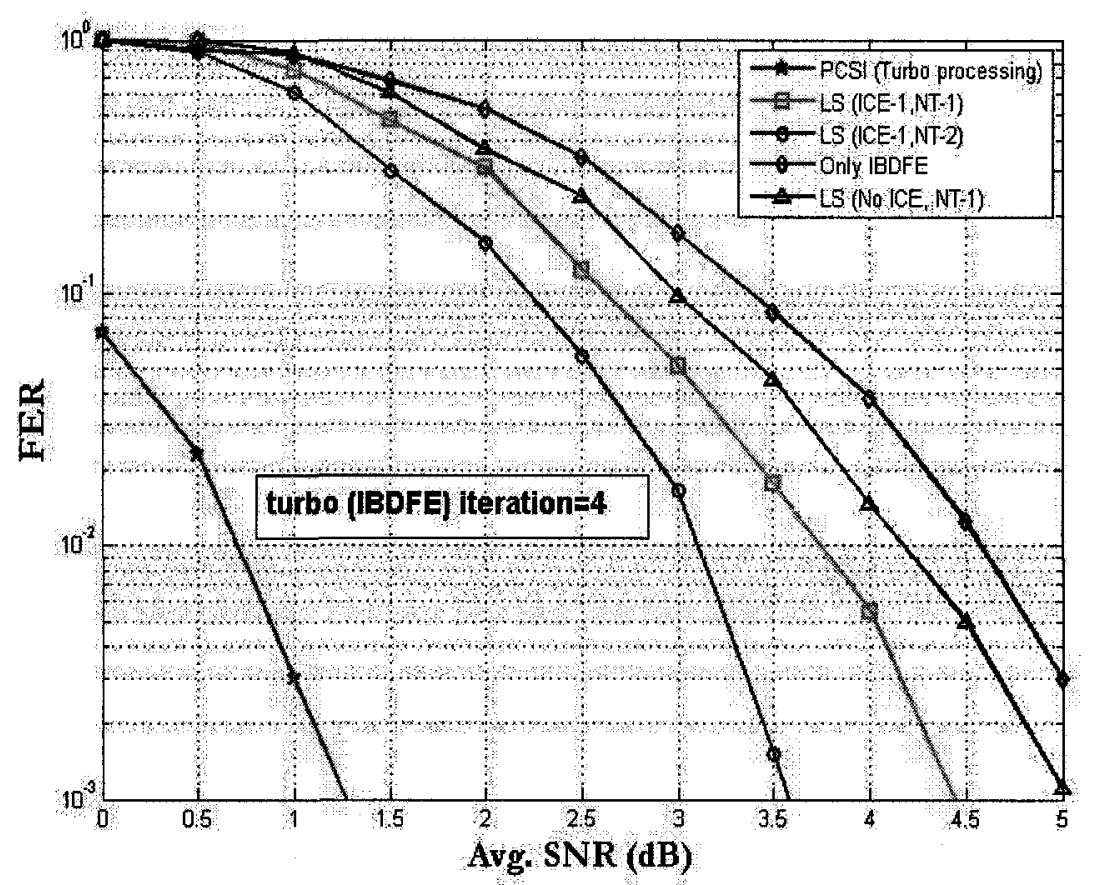

Fig. 7.3. Average FER-Comparison of different algorithms with $\mathrm{TE}$ with $I_{\mathrm{ICE}}=1, I_{\mathrm{E}}=4$, $K=2, P=4 @-15 \mathrm{~dB}, N T=1$ and $2, M=3$ and $T_{r}=384$.

Figure 7.4 shows the effect of more HD-DFICE iterations on the FER performance. Error propagation causes worse performance of HD-DFICE with 2 iterations at low SNR levels. A crossover happens at around $3.6 \mathrm{~dB}$. This trend shows that further iterations may help at or after $3.6 \mathrm{~dB}$ of SNR. 


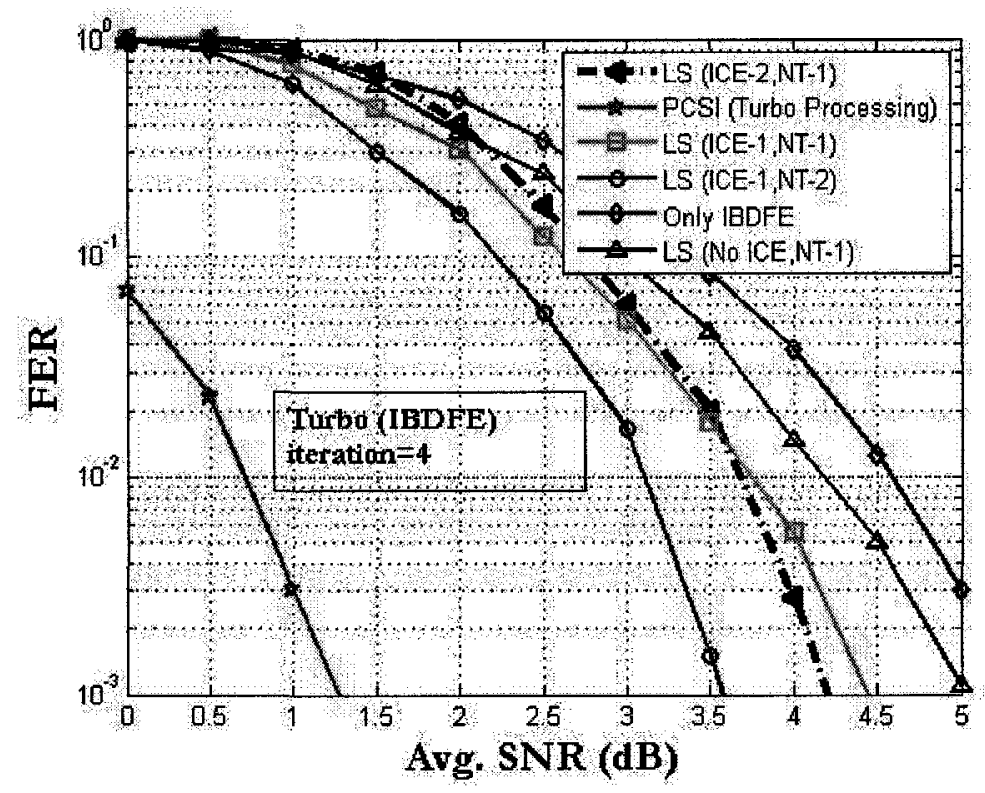

Fig. 7.4. Average FER-Comparison of different algorithms with TE with $I_{\mathrm{ICE}}=1$ and $2, I_{\mathrm{E}}$

$$
=4, K=2, P=4, N T=1 \text { and } 2, M=3 \text { and } T_{r}=384 \text {. }
$$

The fraction of pilot overhead is computed as:

$$
\text { Pilot Overbead }=\left(\frac{\# \text { of training }- \text { carrying blocks } / \text { frame } * \operatorname{Tr}^{*} N T^{*} K}{1152 \text { symbols } / \text { block } * 12 \text { blocks } / \text { frame }}\right)
$$

Figure 7.5 illustrates the effect of reducing the pilot overhead. With $N T=2$ around $16.7 \%$ ( $\operatorname{Tr}=288, B=1152$ ) overhead consumed by the pilots, compared with the previous $22.2 \%$ ( $\operatorname{Tr}=384, B=1152$ ) case. It is observed, that still performance of within $2.5 \mathrm{~dB}$ SNR degradation with TE, and interference suppression via LS adaptation compared to the PCSI, is achievable. 


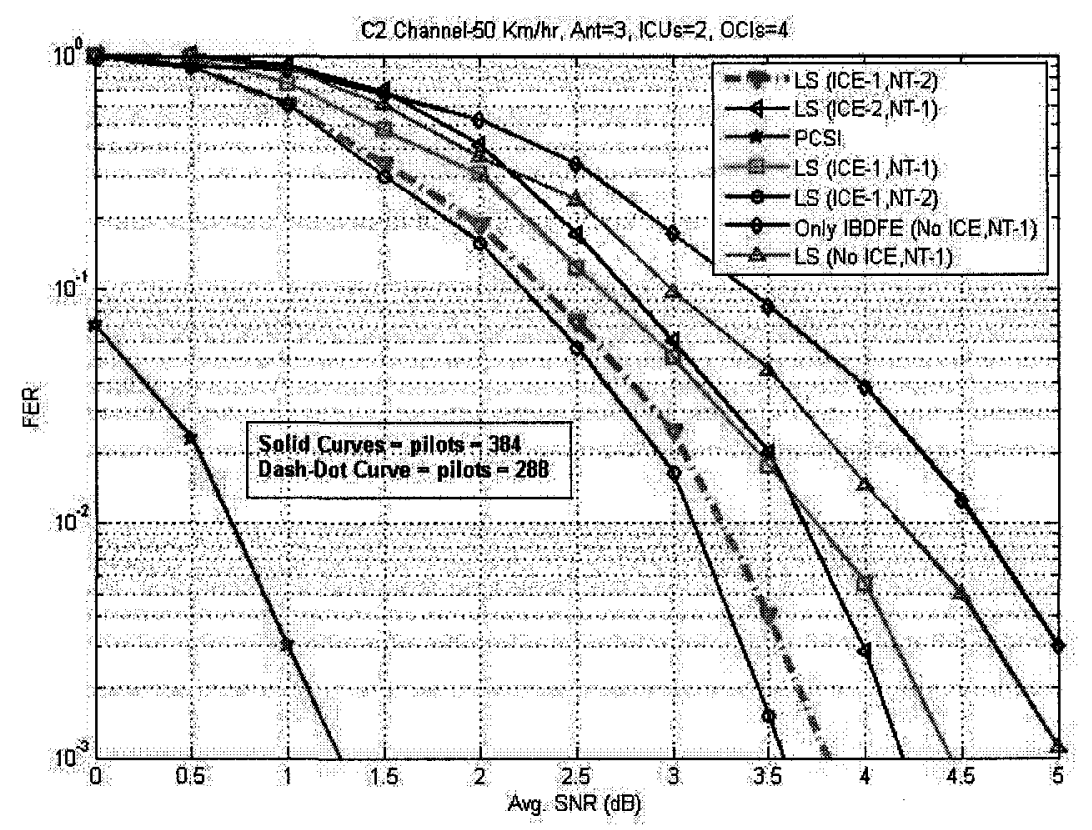

Fig. 7.5. Average FER-Comparison of different algorithms with TE with $I_{\mathrm{ICE}}=1$ and $2, I_{\mathrm{E}}$ $=4, K=2, N T=1$ and $2, P=4, M=3$ and $T_{r}=288$ and 384 .

Increased diversity gain improves the decisions and an SNR improvement of around $1.6 \mathrm{~dB}$ is achieved by increasing the number of elements at the BS from $M=3$ to $M=4$ with 16.7\% pilot overhead. 'M3' and 'M4' in Figure 7.6 represent 3 and 4 BS antenna elements respectively. The distant OCIs with reduced received relative power levels (@$18.2 \mathrm{~dB}$ ) reduce the noisy effect on the composite received signal and the CE performance improves. The average FER performance with such low power OCIs is also shown in Figure 7.6 (dashed lines). 


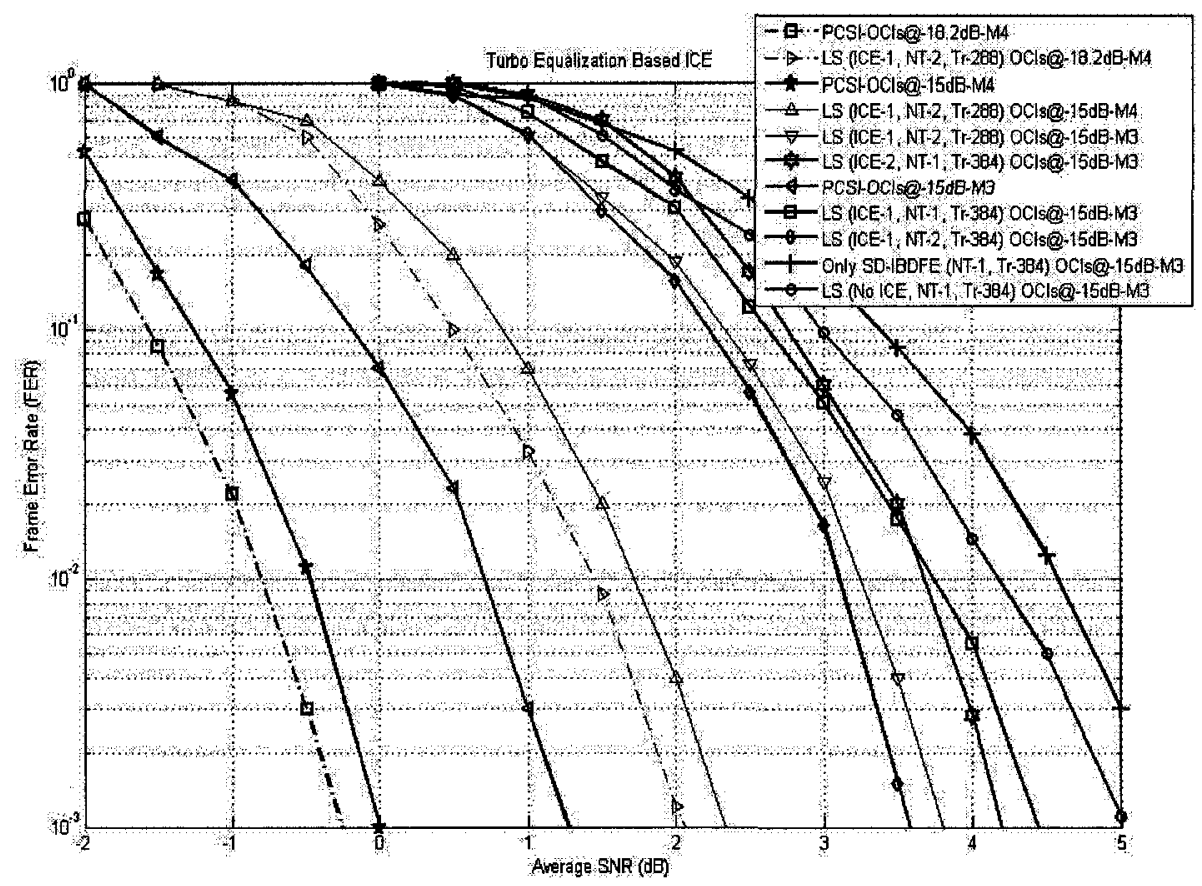

Fig. 7.6. Average FER-Comparison of different algorithms with TE with $I_{\mathrm{ICE}}=1$ and $2, I_{\mathrm{E}}$ $=4, K=2, P=4, M=3$ and 4 and $T_{r}=288$ and 384 .

\subsection{Concluding Remarks on TE with HD-DFICE}

Table 7.2 summarizes the TE with HD-DFICE performance at a FER of $10^{-2}$ with $M=4$, $K=2, I_{\mathrm{ICE}}=1$ or $2, N T=1$ or 2 and with $(P=4 @-15 \mathrm{~dB})$ or without OCIs $(P=0)$. The table contains results from Figure 7.6 and also some new results, which are not shown graphically in any figure. Numbers (not bold) shows the absolute SNR while numbers shown in (bold) represents the SNR degradation caused by the imperfect CE. The second column in the table represents the absolute SNR with PCSI with and without the presence of the OCIs. The last three columns are dedicated to the $\mathrm{CE}$ algorithms. The first $\mathrm{CE}$ column shows the results with the receiver utilizing only non-iterative $\mathrm{CE}$ based on SoISoO-IBDFE. The second and third CE columns show the results with HD-DFICE without and with LS-IS respectively.

It is observed that compared to the LS-IS algorithm presented in Chapter 6, the addition of TE reduces required SNR by 1 to $3 \mathrm{~dB}$. SNR degradations due to non-perfect CSI remain at $2-4 \mathrm{~dB}$. 
Table 7.2. Summary of the comparison between different algorithms with TE, $M=4, K=2, P=4, N T=1$ and $2, I_{\mathrm{ICE}}=1$ and 2 .

\begin{tabular}{|c|c|c|c|c|}
\hline \multirow{2}{*}{$\begin{array}{l}\text { Turbo-Results } \\
B=1152 \\
T_{r}(1 \quad \& 12 \text { symbol })=288 \\
\text { If } N T=1, I_{I C E}=2 \\
\text { If } N T=2, I_{I C E}=1\end{array}$} & \multirow{2}{*}{$\begin{array}{l}\text { SNR for PCSI } \\
\text { (dB) for } 10^{-2} \\
\text { FER }\end{array}$} & \multicolumn{3}{|c|}{$\begin{array}{l}\text { SNR with channel estimation (dB) (SNR } \\
\text { degradation due to non-perfect CSI shown in } \\
\text { bold) }\end{array}$} \\
\hline & & $\begin{array}{c}\text { Non-iterative } \\
\text { CE } \\
\text { SoISoO- } \\
\text { IBDFE }\end{array}$ & $\begin{array}{c}\text { DFICE } \\
\text { No LS-IS }\end{array}$ & $\begin{array}{l}\text { DFICE with } \\
\text { LS-IS }\end{array}$ \\
\hline No OCIs $(N T=1)$ & -0.8 & $2.9(3.7)$ & $1.9(2.7)$ & $1.6(2.4)$ \\
\hline No OCIs $(N T=2)$ & -0.8 & $1.9(\mathbf{2 . 7})$ & $1.2(\mathbf{2 . 0})$ & $0.9(\mathbf{1 . 7})$ \\
\hline $\begin{array}{c}4 \text { OCIs, each at }-15 \mathrm{~dB} \\
(N T=1)\end{array}$ & -0.4 & 3.7 (4.1) & $2.9(\mathbf{3 . 3 5})$ & $2.25(2.65)$ \\
\hline 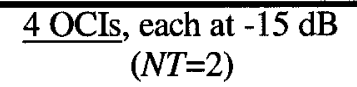 & -0.4 & $2.7(3.1)$ & $2.1(2.5)$ & $1.7(\mathbf{2 . 1})$ \\
\hline
\end{tabular}

\subsection{TE-assisted Sub-Space Interference Suppression (SS-IS)}

Unknown out of cell interference can be suppressed with a combination of a sub-spacebased (SS) method and PIC technique [RW02]. Here we are proposing that in a SC-FDE uplink SDMA system, out of cell interference can be filtered out by a Sub-Space (SS) technique with the help of the proposed TE-assisted LS adaptation while PIC suppresses the in-cell Interference. This can also be combined with a subsequent DFICE. We name this Sub-Space IS (SS-IS) approach. In the SS-IS method, an unknown interference signal plus noise sub space is estimated, and the estimate is projected onto the SS to get a cleaner version of the known users. In SS-IS we will be utilizing the previously proposed interference suppression techniques along with the SS-IS method to suppress the OCIs.

\subsubsection{SS-IS Algorithm}

In this algorithm, the objective is to improve the channel estimates of the $K$ ICUs. Decoder hard decisions on interfering ICUs' data (obtained after several SoISoO-IBDFE 
iterations) are used to remove the interference. From hard decisions of the ICUs and the estimated CFRs, a preliminary estimate of the overlapping unknown OCIs is obtained. At the $j$ th DFICE iteration such that $j=1,2, \ldots, I_{\text {ICE }}$, at $n$th frequency and after $I_{E}$ iterations of SoISoO-IBDFE such as $i=0,1,2, \ldots, I_{\mathrm{E}}-1$, the effect of OCIs at the array output can be estimated as [RW02]:

$$
\nabla^{j}[n]=\mathbf{Y}[n]-\sum_{k=1}^{K} \hat{\mathbf{H}}_{k}^{j-1}[n] \tilde{S}_{k}^{j}[n]
$$

where, $\nabla^{j}[n] \in \mathrm{C}^{M \times 1}$ and $\mathrm{Y}[n]$ is the array input. Note that the summation is over all the ICUs $(K) . \nabla^{j}[n]$ contains a rough estimate of the OCIs plus the additive noise. Because of the CE and decision errors of the ICUs, there is some residual ICUs' effect left in $\nabla^{j}[n]$. Ideally, with perfect decisions, $\nabla^{j}[n]$ will only contain OCIs effects and additive noise. The correlation matrix of the OCIs' plus noise is given by: $\Gamma[n]=E\left[\nabla^{j}[n] \nabla^{j}[n]^{\mathrm{H}}\right]$, where $\Gamma[n] \in C^{M \times M}$. In practice, $\Gamma[n]$ is estimated by time averaging over several SC-FDE blocks (over $F$ blocks per frame, $c=1,2, \ldots, F$ ), that is:

$$
\hat{\Gamma}[n]=\frac{1}{F}\left[\sum_{c=1}^{F} \nabla^{j}[n, c] \nabla^{j}[n, c]^{H}\right]
$$

The eigenvalue decomposition of the estimated time averaged OCIs plus noise correlation matrix $\hat{\Gamma}[n]$ gives a unitary matrix $\mathbf{U}_{\mathrm{OCI}}$ with the $P$ largest eigenvectors of the

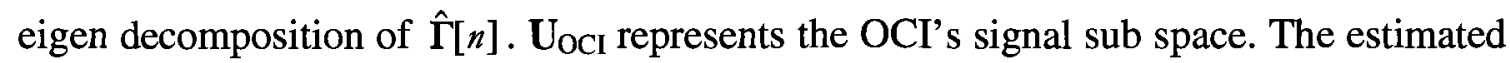
vector of the OCIs, $\overrightarrow{\mathbf{Q}}_{\text {oCl }}$, is obtained by projecting $\nabla^{j}[n]$ onto $\mathbf{U}_{\text {ocl }}$.

$$
\overline{\mathbf{Q}}_{O C I}[n]=\mathbf{U}_{O C I} \mathbf{U}_{O C I}^{H} \nabla^{j}[n]
$$

Estimation of the OCI free array input is established by subtracting OCI's estimates from the received vector:

$$
\boldsymbol{\Psi}^{j}[n]=\mathbf{Y}[n]-\overrightarrow{\mathbf{Q}}_{O C I}[n]
$$

$\boldsymbol{\Psi}^{j}[n]$ is an estimate of the 'out of cell interference free' array input. Then PIC is applied to remove in-cell interference:

$$
\mathbf{X}_{k}^{j}[n]=\Psi^{j}[n]-\sum_{\substack{\ell=1, \ell \neq k}}^{K} \hat{\mathbf{H}}_{l}^{j}[n] \widetilde{S}_{l}^{j}[n]
$$


where $\mathbf{Y}[n]$ is the array output and $\tilde{S}$ denotes hard decisions. The quality of the $\Psi^{j}[n]$ highly depends on the decisions and eventually the estimated CFRs of the ICUs. $\mathbf{X}_{k}^{j}[n]$ obtained in (7.17) is used to update the CFR estimates.

\subsubsection{Simulation Results}

The simulation parameters and the interference distribution considered are the same as described in Section 7.5.1. Simulations are carried out with four antenna elements, four OCIs and two ICUs. For OCIs, two different received average power levels of $15 \mathrm{~dB}$ and $18.2 \mathrm{~dB}$ below the ICUs are considered. SS-IS algorithm is applied and results from LSIS with TE are also plotted and marked as LS-IS. Figure 7.7 illustrates that there is a 0.25 $\mathrm{dB}$ improvement beyond the LS-IS results of the previous section, with the employment of the SS-IS. The performance of SS-IS with OCIs at $-15 \mathrm{~dB}$ is comparable to the LS-IS with TE performance with OCIs at $-18.2 \mathrm{~dB}$ at a FER of $10^{-2}$. The PCSI and no-OCI case with LS-IS with TE plots are also shown for comparison purposes.

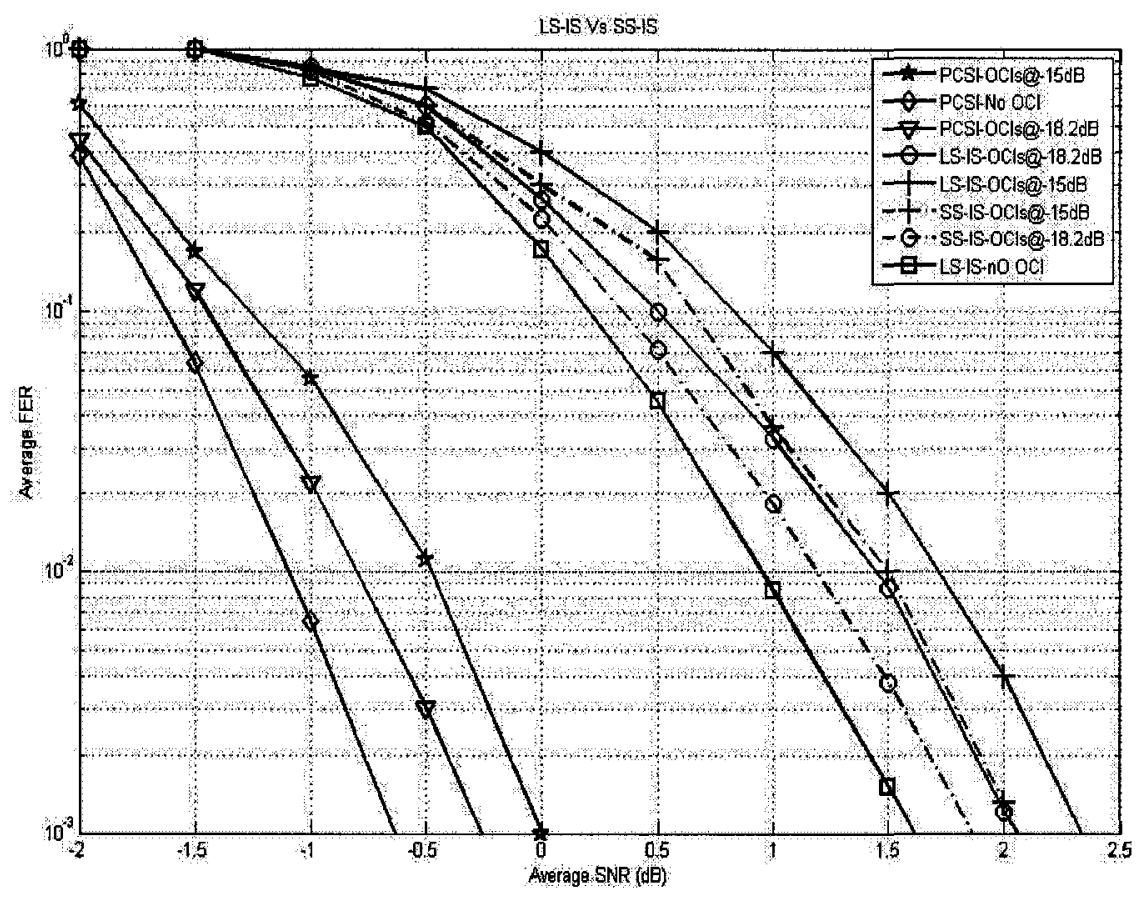

Fig. 7.7. Average FER-Comparison of LS-IS and SS-IS with TE and DFICE, $I_{\mathrm{E}}=4, I_{\mathrm{ICE}}$

$$
=1, N T=2, M=4 \text { and } T_{r}=288 \text {. }
$$




\subsection{Frequency Domain TE-based SD-DFICE}

So far the hard decisions from the decoder output are used in DFICE. Although the use of the hard decisions in DFICE gives a low complexity solution for ICE it is sub-optimum. Instead DFICE driven by the extrinsic information (LLRs) or soft decisions may improve the CE performance because it takes the reliability of the decisions into account. Moreover, results with the 'all pilot transmission' in Chapter 6 also suggest that there is still considerable room for improvement in the decision directed algorithms like PIC, DFICE or LS adaptation. In this section we apply soft decisions in the proposed threshold-based DFICE with the turbo processing and denote it as Soft Decision-based DFICE (SD-DFICE). A simplified block diagram is shown in Figure 7.8.

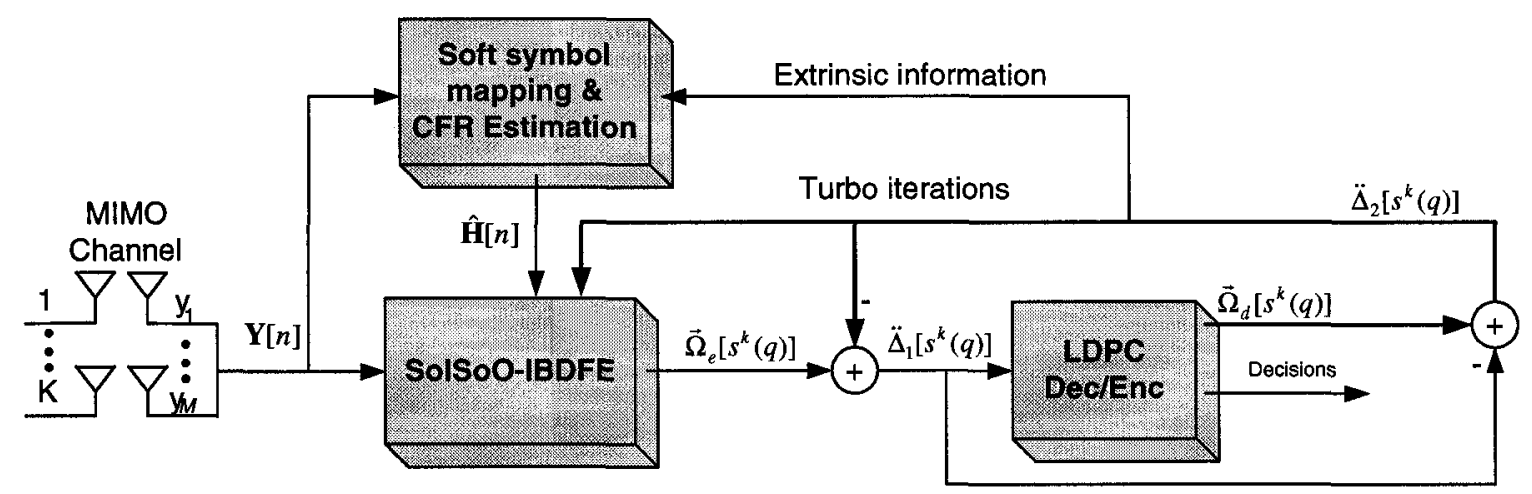

Fig. 7.8. Simplified block diagram of soft decision based DFICE.

In DFICE approaches the use of soft decisions and the incorporation of the code structure are two useful components, which can be utilized to mitigate the error propagation. The LDPC decoder can supply soft decisions and implement the code structure at the same time. The LDPC code uses an iterative decoder, and hence combines more naturally with ICE. In the next section, we present a SC-FDE receiver that combines LDPC-based turbo decoding with ICE via the Expectation Maximization (EM) algorithm [ZP99]. 


\subsubsection{Derivation of SD-DFICE using EM Algorithm}

Using soft decisions in DFICE, in which the estimator is derived from Expectation Maximization (EM) principle [KV95] can reduce error propagation and improve the DFICE performance. We consider the EM algorithm [KV94] for Soft Decision-based DFICE (SD-DFICE) for the $k$ th ICU. Before the decoding process is completed, $S_{k}$ is unknown, where, $S_{k}$ represents the $k$ th user's data symbol; however we have some $a$ priori knowledge on the unknown $S_{k}$. The EM algorithm is a suitable choice to exploit the a-priori knowledge and if it converges is close to be the ML solution. Maximizing a likelihood function approaches the ML solution, but since the $S_{k}$ in the function is unknown, the two-step EM algorithm "maximizes" the "expectation" of the loglikelihood function instead, over all possible $S_{k}$. It starts from a pilot-based initial channel estimates to estimate $S_{k}$, which is then used to further refine the channel. In each iteration the a-priori constraints are enforced in the step of estimating $S_{k}$. When only the probabilities, not the exact symbols are available the channel estimates are still close to a ML solution and can be written as:

$$
\hat{\mathbf{H}}_{k_{-}}=\arg \max _{\mathbf{H}_{k}} \log p\left(\mathbf{H}_{k} \mid S_{k}, \mathbf{Y}\right)
$$

where $p(x)$ denotes probability of an event $x$. As $S_{k}$ is not known, there is no closed form solution to (7.18) and the iterative EM algorithm, which maximizes instead an averaged form of the likelihood function using expectation-maximization is well suited for this type of problem. In EM terminology if both $\mathbf{Y}$ and $S_{k}$ are known a set $\mathbf{U}_{k}=\left\{\mathbf{Y}, S_{k}\right\}$ is known as a "complete data set" and the parameter set of interest is $\hat{\mathbf{H}}_{k}$. At the $j$ th EM or DFICE iteration, the maximization of the expectation procedure is expressed as:

$$
\hat{\mathbf{H}}_{k}^{j+1}=\arg \max _{\mathbf{H}_{k}}\left[\left(\log p\left(\mathbf{H}_{k}\right)+E_{S_{k} \mid \mathbf{Y}, \mathbf{H}_{k}^{j}}\left[\log p\left(\mathbf{Y} \mid S_{k}, \mathbf{H}_{k}\right)\right]\right.\right.
$$

Assuming the CFR at frequency $n$ for $k$ th user is a complex Gaussian random variable with mean given by the pilot-based estimate and variance $\sigma_{H_{k}}^{2}$, its density function is

$$
p\left(\mathbf{H}_{k}\right)=\frac{1}{\pi \sigma_{H_{k}}^{2}} \exp \left(-\frac{1}{\sigma_{H_{k}}^{2}}\left|\mathbf{H}_{k}-\hat{\mathbf{H}}_{k}^{0}\right|^{2}\right)
$$


In these derivations frequency index $n$ is omitted for simplicity. In (7.20), $\sigma_{H_{k}}^{2}$ is the variance of the complex $\hat{\mathbf{H}}_{k}$ and $\hat{\mathbf{H}}_{k}^{0}$ is the $k$ th user initial pilot-based channel estimate. Also,

$$
E_{S_{k} \mid \mathbf{Y}, \mathbf{H}_{k}^{\prime}}\left[\log p\left(\mathbf{Y} \mid S_{k}, \mathbf{H}_{k}\right)\right]=\log \left(\frac{1}{\pi \sigma_{n}^{2}}\right)-\frac{1}{\sigma_{n}^{2}} \sum_{\text {All } S_{k}}\left|S_{k} \mathbf{H}_{k}-\mathbf{Y}\right|^{2} p\left(S_{k} \mid \mathbf{Y}, \mathbf{H}_{k}\right)
$$

By discarding all the terms that do not depend on the $\mathbf{H}_{k}$, we obtain

$$
\hat{\mathbf{H}}_{k}^{j+1}=\arg \max _{\mathbf{H}_{k}}\left[-\frac{1}{\sigma_{\mathbf{H}_{k}}^{2}}\left|\mathbf{H}_{k}-\hat{\mathbf{H}}_{k}^{0}\right|^{2}-\frac{1}{\sigma_{n}^{2}} E_{S_{k} \mid \mathbf{Y}, \mathbf{H}_{k}^{\prime}} \sum_{A \mu \mathbf{S}_{k}}\left|\sum_{m \neq k} S_{m} \hat{\mathbf{H}}_{m}+S_{k} \hat{\mathbf{H}}_{k}-\mathbf{Y}\right|^{2}\right](7 .
$$

By setting the gradient with respect to $\mathbf{H}_{k}$ equal to zero, we get

$$
-\frac{1}{\sigma_{\mathbf{H}_{k}}^{2}}\left(\hat{\mathbf{H}}_{k}^{j+1}-\hat{\mathbf{H}}_{k}^{0}\right)-\frac{\hat{\mathbf{H}}_{k}^{j+1}}{\sigma_{n}^{2}} E_{S_{k} \mid \mathbf{Y}, \mathbf{H}_{k}^{j}} \sum_{m \neq k}\left|S_{k}\right|^{2}+\frac{1}{\sigma_{n}^{2}} E_{\mathbf{S}_{k} \mid \mathbf{Y}, \mathbf{H}_{k}^{\prime}}\left[\mathbf{S}_{k}^{*}\right]\left(\mathbf{Y}-\sum_{m \neq k} S_{m} \hat{\mathbf{H}}_{m}\right)=0(7
$$

Which gives us

$$
\hat{\mathbf{H}}_{k}^{j+1}=\frac{\hat{\mathbf{H}}_{k}^{0}+\frac{\sigma_{H_{k}}^{2}}{\sigma_{n}^{2}} E_{\mathbf{s}_{k} \mid \mathbf{Y}, \mathbf{H}_{k}^{j}}\left[S_{k}^{*}\right]\left(\mathbf{Y}-\sum_{m \neq k} S_{m} \hat{\mathbf{H}}_{m}\right)}{1+\frac{\sigma_{H_{k}}^{2}}{\sigma_{n}^{2}} E_{S_{k} \mid \mathbf{Y}, \mathbf{H}_{k}^{j}}\left[\left|S_{k}\right|^{2}\right]}
$$

The denominator of (7.24) contains the autocorrelation (second moment) of the sequence $S_{k}$, i.e. $E\left(\left|S_{k}\right|^{2}\right)$, and in the numerator we have the expectation (first moment) $E\left(S_{k}^{*}\right)$ of the sequence, often called the soft decision. The expectation is taken over all possible $S_{k}$ that are constrained by the code structure.

The problem of noise enhancement in SC-FDE systems while computing the CFR via decisions is dealt with the FRT algorithm described in Chapter 6. In FRT the noise enhancement affected CFRs are replaced by either the CFRs obtained in the previous ICE iteration i.e. $\hat{\mathbf{H}}_{k}^{j-1}$ or with the pilot-based initial CFRs i.e. $\hat{\mathbf{H}}_{k}^{0}$. A rationale of the FRT is provided by (7.24). The estimate obtained from (7.24) tends to avoid the noise enhancement problem inherent in SC-FDE due to frequency selectivity in $S_{k}$. For small values of $S_{k}$, the estimate in (7.24) is dominated by $\hat{\mathbf{H}}_{k}^{0}$.

The iterative channel estimator can use either hard decisions with FRT using (6.4 with no LS-IS), (6.6 with LS-IS) or soft decisions where the soft decision based estimator is 
derived from the EM principle using (7.24). Both hard and soft decisions-based estimators use the pilots and 2x1D interpolation estimators to obtain the initial channel estimates. The full-length estimated CFR is passed through a low pass smoothing filter described in Chapter 2 to reduce the effects of noise and OCIs. In the smoothing process the full length CFR is converted into the time domain by using an IFFT and smoothing is done by setting to zero all the impulse response samples beyond the maximum span of $L$, where $L$ is the channel memory length.

\subsubsection{Simulation Results}

The tradeoff between complexity and the decoded FER performance is characterized in simulations by comparing the hard and soft decision-based iterative channel estimator results.

Figures 7.9 and 7.10 illustrate the average FER results with Soft Decision-based DFICE (SD-DFICE) using (7.24) while HD-DFICE results are also shown for comparison purposes. Figure 7.9 illustrates results with no inter-cell interference while Figure 7.10 includes the OCIs' effect. Simulations are carried out with 4 antenna elements $(M=4), 2$ ICUs $(K=2), 4$ or zero OCIs $(P=4$ or 0$), 4$ SoISoO-IBDFE iterations $\left(I_{\mathrm{E}}=4\right)$, upto 3 DFICE iterations ( $I_{\mathrm{ICE}}=1,2$, and 3) with $2000 \mathrm{C} 2$-channel realizations. An SNR gain of about $1.5 \mathrm{~dB}$ is achieved over the hard decision-based DFICE in a system with 4 low power $(-15 \mathrm{~dB})$ OCIs. With 3 SD-DFICE iterations, at the FER of $10^{-2}$, the SNR degradation with $\mathrm{CE}$ is only about $0.6 \mathrm{~dB}$ with respect to the PCSI. Almost the same amount of SNR gain is achieved with the scenario of having only in-cell interference. In comparison with Figures 7.6 and 7.7, Figures 7.9 and 7.10 illustrate that the soft decisions based on the extrinsic information can be utilized in the DFICE to obtain enhanced $\mathrm{CE}$ performance.

Hence the SD-DFICE algorithm could be considered as the "ultimate" equalization and CE technique developed and evaluated in the thesis. Referring to Figure 7.10, it exceeds the next-best (HD-DFICE) performance by $\sim 1.5 \mathrm{~dB}$ and it comes within about $0.5 \mathrm{~dB}$ of TE with PCSI at low (0 to $0.5 \mathrm{~dB})$ SNRs per antenna. 


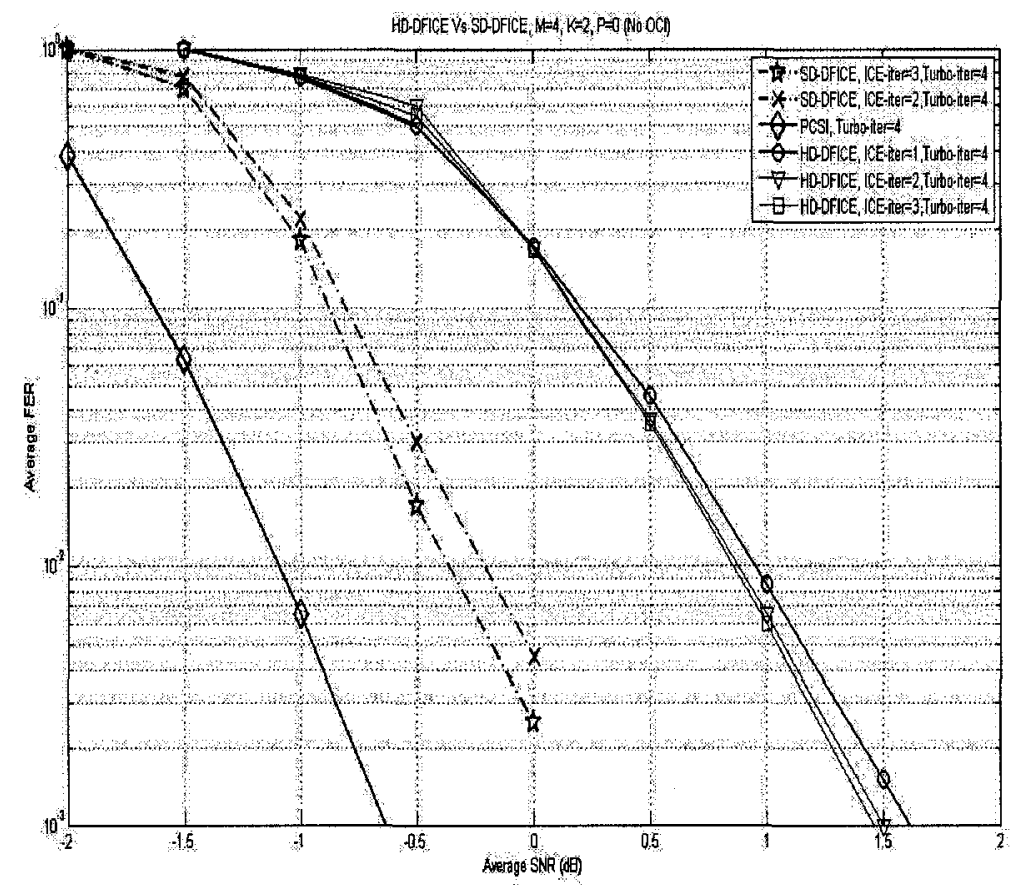

Fig. 7.9. Average FER-Comparison of hard decision and soft decision-based DFICE, SSIS and LS-IS with TE, $I_{\mathrm{ICE}}=1,2$ and $3, N T=2, F_{\Gamma}=1, M=4=I_{\mathrm{E}}, P=0$ and $T_{r}=288$.

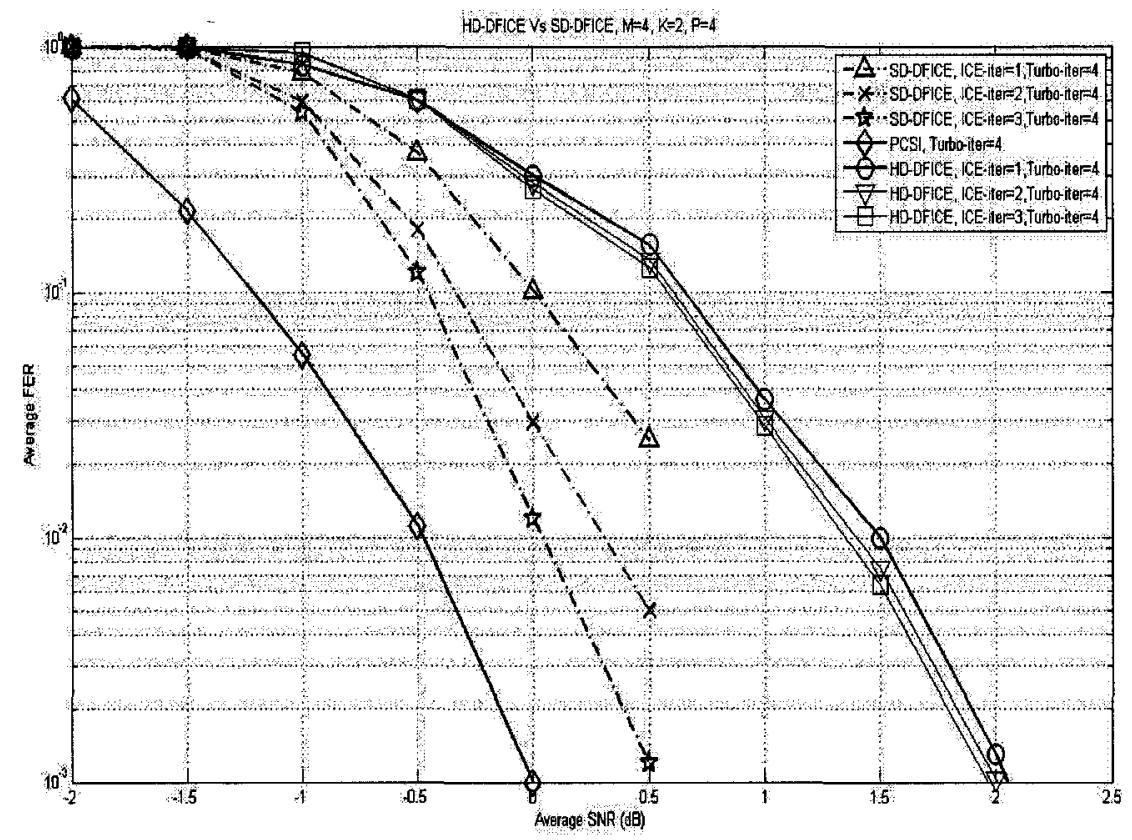

Fig. 7.10. Average FER-Comparison of hard decision and soft decision-based DFICE, SS-IS and LS-IS with TE, $I_{\mathrm{ICE}}=1,2,3$ and $4, N T=2, I_{\mathrm{E}}=4$ and $5, F_{\mathrm{r}}=1, M=P=4 @-15 \mathrm{~dB}$ and $T_{r}=288$. 


\subsection{Complexity Analysis}

In ICE and interference suppression processes, there are three iteration cycles involved. First is the LDPC decoder internal iterations, second is the turbo (IBDFE) iterations and the last is the ICE iterations. These three types of iterations drive the complexity of the overall ICE process. The overall complexity of the receiver processing in terms of the number of complex multiplications is broken into different stages of the CE process. The complexity associated with the matrix inversion highly depends on the numerical method used to compute the inversion. For a matrix of size $M$, associated complexity with efficient matrix inversion is around $M^{3}$. (freq) ${ }^{3}$ and (time) ${ }^{3}$ denote the complexity associated with the matrix inversion of the pre-computed interpolation matrices in frequency and time respectively. The dimension of these matrices depends on the number of pilots in the sliding window used in the time-frequency interpolation.

There is one FFT required for computing the frequency domain CFR. For PACE and interpolation, two matrix inversions (in time and frequency axis) are required to be computed. But these matrices can be pre-computed and do not contribute in the on-line computational complexity. SoISoO-IBDFE involves one matrix inversion, two FFTs and three multiplications. Similarly, PIC needs one multiplication. With LS-IS, one additional matrix inversion, two multiplications and one FFT/IFFT are added in the overall complexity equation. SS-IS adds two multiplications in the overall complexity. DFICE also contributes in the overall complexity with additional one multiplication and one FFT/IFFT. Table 7.3 summarizes the complexity associated with different algorithms.

Table 7.3. Number of complex multiplications per ICE iteration $\left(I_{\text {ICE }}\right)$ per ICU.

\begin{tabular}{|c|c|}
\hline Process/Algorithm & Associated Computational Complexity \\
\hline $\begin{array}{c}\text { Frequency Domain Channel Transfer } \\
\text { Function }\end{array}$ & $B\left[\log _{2}(B)\right]$ \\
\hline Initial CE/Interpolation & $(\text { freq })^{3}+(\text { time })^{3}+B+B\left[(M)^{3}+1\right]$ \\
\hline SoISoO-IBDFE & $I_{\mathrm{E}} B\left[(M)^{3}+2 \log _{2}(B)+3\right]$ \\
\hline PIC & $B$ \\
\hline LS-IS Forward Processing & $B\left[(M)^{3}+2+\log _{2}(B)\right]$ \\
\hline SS-IS Forward Processing & $2 B$ \\
\hline DFICE processing & $B\left[1+\log _{2}(B)\right]$ \\
\hline
\end{tabular}




\subsection{Summary}

In this chapter, interference mitigation algorithms presented in Chapter 6 are modified with the inclusion of turbo processing. A turbo equalization-assisted Soft Decision-based, iterative multi-user CE technique (SD-DFICE) is presented. We use orthogonal pilots, a 2x1D interpolator and a smoothing filter to obtain reasonable initial channel estimates. On the other hand in the decision directed mode, soft symbols obtained via turbo processing are much more effective in the DFICE process compared to the hard decisions. The proposed SD-DFICE scheme is supported by a combination of adaptation and interference suppression algorithms. Moreover, a Sub-Space based method (SS-IS) is also introduced to suppress the OCIs explicitly. It is shown that PIC assisted SoISoOIBDFE suppresses the intra-cell interference along with the ISI, while the LS-IS and SSIS algorithms are useful in suppressing the effects of OCIs. An EM-based justification of the proposed multi-user threshold based frequency replacement DFICE technique is also presented. As in Figure 7.10, the SD-DFICE technique performs the best and exceeds the HD-DFICE algorithm by around $1.5 \mathrm{~dB}$ and the SNR degradation with respect to the PCSI is only $0.5 \mathrm{~dB}$. 


\section{Chapter 8}

\section{Chunk-Based Transmission in SC-FDE}

\section{SDMA systems}

\subsection{Introduction}

In this chapter, the multi-user SD-IBDFE and HD-DFICE is applied to suppress the intracell and inter-cell interference in a split-spectrum or chunk-based SC-FDE system. A chunk-based system introduced under the WINNER concept, named Block Interleaved Frequency Division Multiple Access (B-IFDMA), is described. For comparison purposes the B-IFDMA is compared with the Full Bandwidth (FBW) system as well as with socalled Local Frequency Division Multiple Access (L-FMDA) system. In a chunk-based system the performance of the CE with orthogonal ICUs' pilots and overlapping OCIs is evaluated under different pilot overheads. The chapter is divided into two parts. The first part describes the general SC-FDE based B-IFDMA signal and system model and its CE performance. The second part addresses the multi-user CE aspects of the L-FDMA system. In the end, results with both systems are compared in a tabular form.

\subsection{B-IFDMA}

Chunk-based communication and relevant radio design is recently receiving interest. A chunk in the WINNER concept is defined as the smallest time-frequency unit, which could be allotted to a user [Win04]. Chunk-based communication allows for flexible use of radio spectrum, and also for enhanced frequency diversity if chunks are separated in frequency by more than the channel's coherence bandwidth. Block Interleaved Frequency Division Multiple Access (B-IFDMA) is a way of resource allocation with equi-distant chunks, recently introduced in WINNER system [SFF07] for non-adaptive uplink transmission for users where instantaneous bit rates are much lower than the total system 
bandwidth. In non-adaptive B-IFDMA the resource/chunk allocation for each user is fixed. B-IFDMA is claimed to be a power-efficient version of a chunk-based communication with reduced frequency offset problem compared to its counter-part, Interleaved Frequency Division Multiple Access (IFDMA) [SBS97]. Power efficiency comes from an intra-chunk sleep mode in which the transmitter is turned off to save power. In B-IFDMA, the usable frequency chunks are called "blocks". The block size is chosen in such a way that it experiences flat fading in its Time-Frequency grid. In this chapter, we will focus on the non-adaptive chunk-based system and its CE aspects.

\subsubsection{Salient features of B-IFDMA}

There are a few advantages claimed for the B-IFDMA system in comparison with the other available access schemes. Some of them are [SFF07]:

- Better frequency diversity compared to the Full Band Width (FBW) and similar frequency diversity provided by the IFDMA.

- Less sensitivity in terms of carrier frequency offset and phase noise compared to the IFDMA.

- Intra-chunk sleep mode is claimed to provide better power efficiency.

The other issues associated with the chunk-based transmission are the following; Keeping the PAPR of the transmitted waveform low, time variation of the chunk-based channel, arrangements and selection of proper training sequences for $\mathrm{CE}$, synchronization, cooperation and competition of multi-users (contention) and multi-user interference management (synchronous and asynchronous ICUs and OCIs). We will be focusing on the $\mathrm{CE}$ aspects of the chunk-based communication in an interference-prone SC-FDE system. For this purpose we apply iterative equalization and CE algorithms described in previous chapters to a B-IFDMA system in inter and intra-cell interference environment with different pilot overheads. Another chunk-based system called Local Frequency Division Multiple Access (L-FMDA) is also considered and compared with B-IFDMA in terms of average FER via computer simulations. 


\subsection{B-IFDMA Structure and System Parameters}

The basic structure of the B-IFDMA proposed in the WINNER system is depicted in Figure 8.1. The system is required to use multiple blocks per user at equally spaced frequency intervals exceeding the maximum expected channel coherence BW to fulfill the data rate requirements. Also in Figure 8.1 'FNU' stands for frequencies not used. In the WINNER Frequency Division Duplex (FDD) wide area transmission mode a chunk is defined as a time-frequency piece of 8 frequencies and 12 blocks $(F=12)$. In previous chapters a FFT block was constituted by a full 416 or 1024 frequencies. In B-IFDMA terminology, "block" is a short time-frequency plane. As shown in Figure 8.1, in basic BIFDMA a block is constituted by 4 frequencies and 3 sub-blocks. Here we define a "subblock" as a block of samples whose time duration is that of a full FFT block (e.g. $25.3 \mu \mathrm{sec}$ in a full system BW of $40 \mathrm{MHz}$ ) but in which only 4 sub-carriers are assigned. A sleep mode consisting of nine sub-blocks is inserted between frames. A sleep mode is the state in which the terminal goes into a power-saving mode by not transmitting. In general the number of symbols per user per frame is given as:

\# of symbols/user $/$ frame $=\#$ of sub-blocks* \# of frequencies $/$ block $*$ \# of blocks $/$ user $/$ frame Assuming an inter-block spacing of 32, the total number of useful symbols per frame will be $3 \times 4 \times 32=384$.

\subsubsection{Pilot-assisted CE in B-IFDMA}

ICUs' frequency-multiplexed pilots are placed orthogonal to each other in the block containing 4 frequencies and 3 sub-blocks $(4 \times 3)$ for initial CE. The pilots of the OCIs overlap the ICUs' pilots. Sleep mode (lasts for nine sub-blocks in this case) concept is also depicted in Figure 8.1. The pilot overhead depends on the number of pilots per block, number of ICUs and the number of training blocks per ICU (NT). The pilot overhead is computed as:

Overbead $=\left(\frac{\# \text { of pilot carying sub-blocks * \# of pilotsymbols per ICU * \# of orthogonal ICUs*\# of short training blocks }}{\text { Total\# of coded symbols / block }}\right)$

Initially the assumption is that each ICU has only one orthogonal pilot per block. The notations $\mathrm{u} 1$ and $\mathrm{u} 2$ in Figure 8.1 represent the orthogonal pilots of the two ICUs (user1 
and user2) in the desired cell. With one pilot in a block, no interpolation is possible in either in time or in frequency axis, and the CFR estimate obtained at the single pilot position for each orthogonal ICU is used for the whole block. With two orthogonal ICUs and $N T=1$, the pilot overhead will be $16.7 \%$ for each ICU. We call this arrangement of pilots with $F=12, F 1-12$.

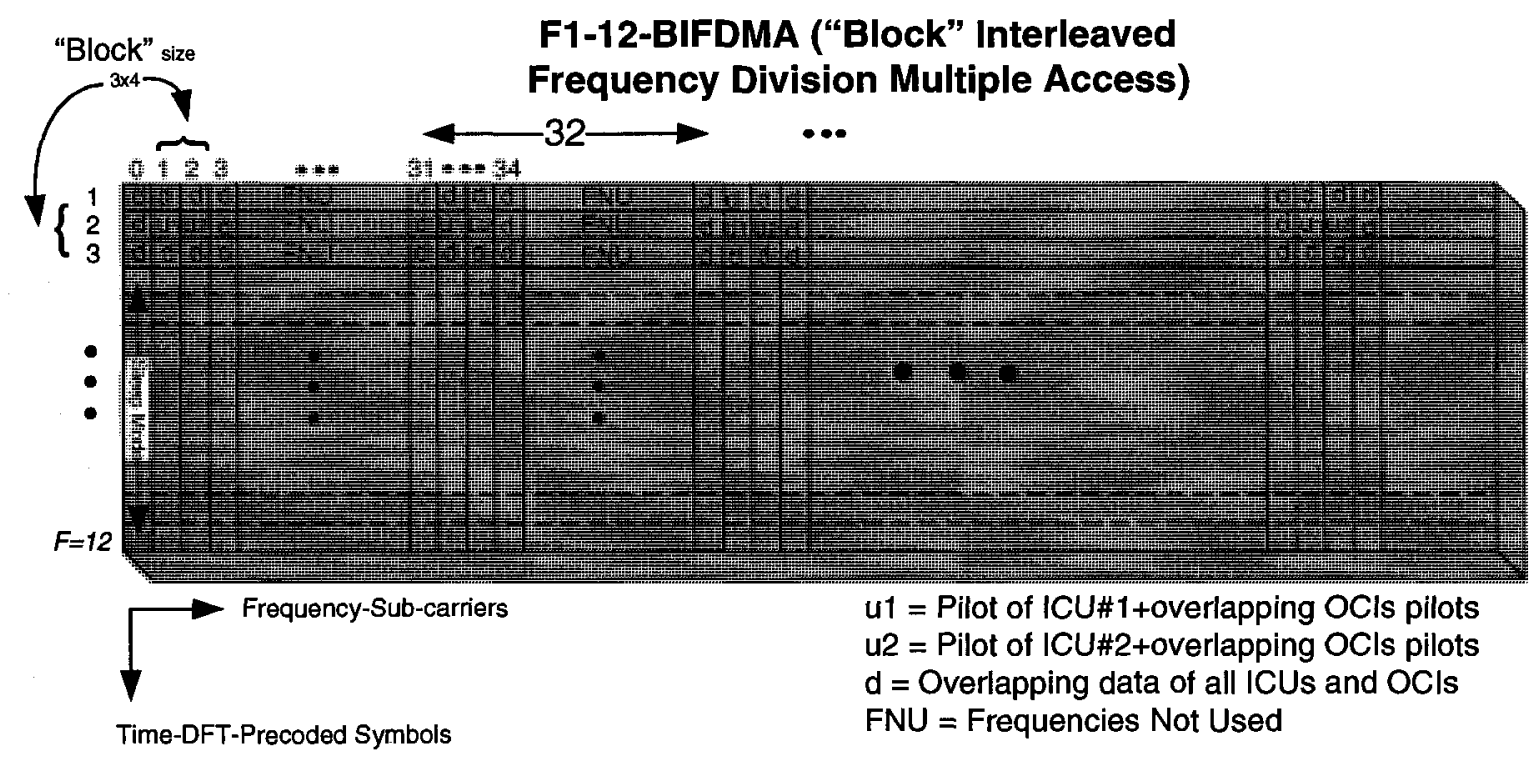

Fig. 8.1. Pilot arrangement for a B-IFDMA chunk-based system with one pilot per user per block-(F1-12).

Figure 8.2 illustrates a comparison of the actual CFR and the estimated CFR with only one pilot per ICU per block. CFR for each ICU is estimated based on only one pilot and due to the wide frequency spacing between blocks and hence the reduced opportunity for frequency domain interpolation, it does not follow the frequency correlation of the actual CFR. From Figure 8.2 we predict that CFR estimated with F1-12 will suffer a large SNR degradation with respect to the PCSI. 


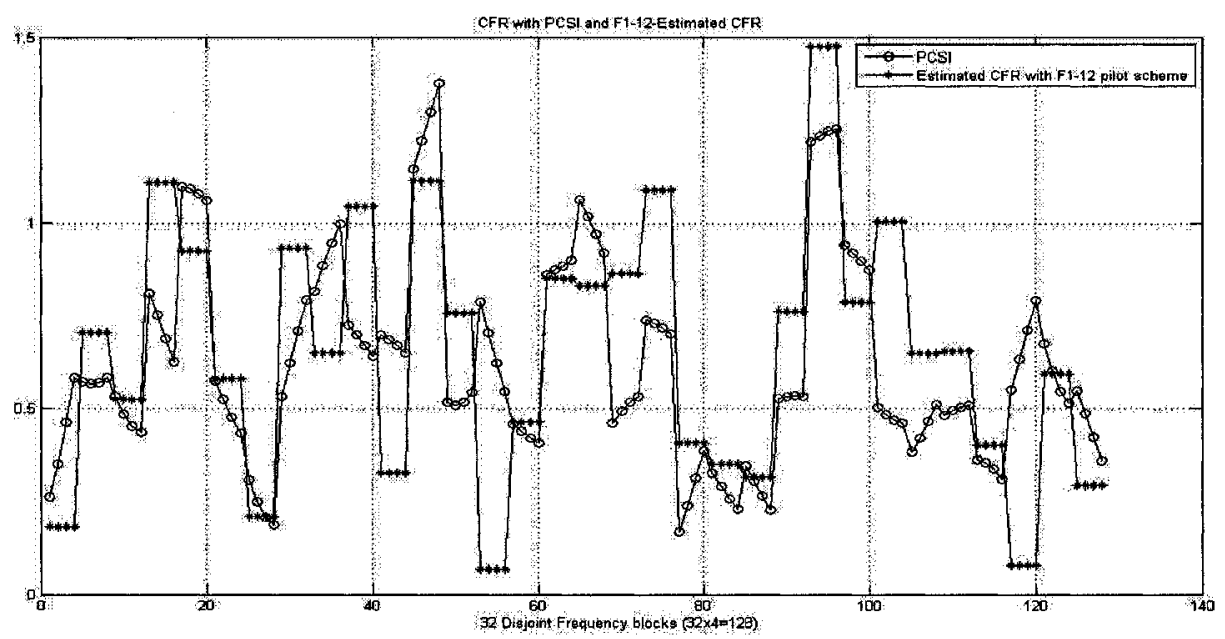

Fig. 8.2. Actual Vs estimated disjoint CFR (straight horizontal lines represent the estimation using F1-12 pilot scheme).

Figure 8.3 shows an attempt to improve the initial CFR estimate at the cost of an additional orthogonal pilot per ICU. Two orthogonal pilots per ICU per block are placed at the edges of the sub-block. Here again we consider two ICUs. First user uses the first sub-block to send its pilots, while the second user sends its pilots in the second sub-block. It should be noted that these pilots are placed orthogonal to each other by placing zeros at the sub-carriers being used as pilots by the other user. The pilot overhead in this case will be $33.33 \%$ for each ICU.

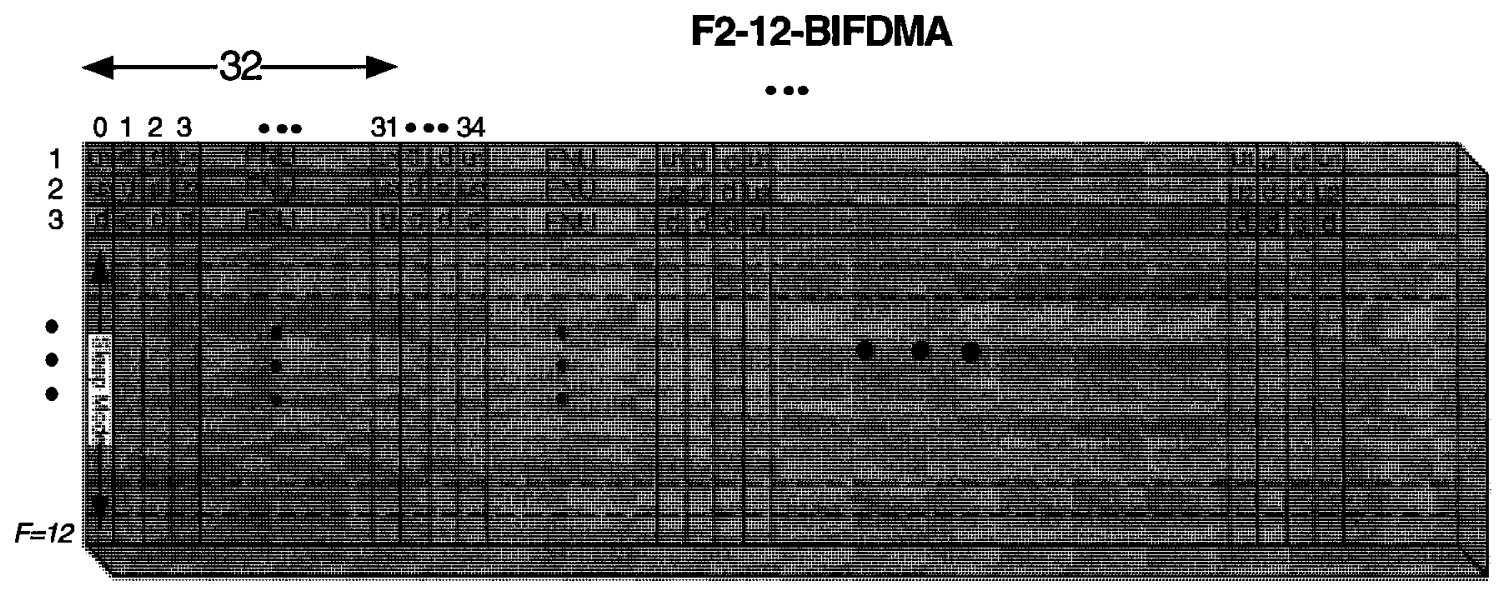

Fig. 8.3. Pilot arrangement for a B-IFDMA chunk-based system with two pilot per user per block -(F2-12). 
Linear Interpolation (LI) is carried out in the frequency direction, and the CFR estimates for the pilot-bearing sub-blocks are obtained. LI for finding the response at any frequency $n$ and at antenna element $m$ is given by:

$$
\hat{H}_{m}^{k}(n)=\hat{H}_{m}^{k}\left(p_{i}\right)+\frac{\hat{H}_{m}^{k}\left(p_{i}+1\right)-\hat{H}_{m}^{k}\left(p_{i}\right)}{p_{i+1}-p_{i}}\left(n-p_{i}\right)
$$

for $p_{i} \leq n \leq p_{i+1}$.

where $\hat{H}_{m}^{k}(n)$ represents the linearly interpolated CFR at the $n$th tone while $\hat{H}_{m}^{k}\left(p_{i}\right)$ denotes the estimated CFR at the $p_{i}$ th pilot tone. With the assumption of slow time variations, the same CFR estimates are used in the remaining two sub-blocks. This pilot placement scheme, named F2-12, is shown in Figure 8.3.

For initial CE, FMT is used and the time averaging to average out the effects of noise and OCIs is obtained by sending more pilot-containing sub-blocks. The proposed pilot arrangement for B-IFDMA with $4 \times 3$ blocks in WINNER is the FMT arrangement of Figure 8.1; i.e. F1-12. For NT>3, a combination of FMT and TMT could be used. As an example, Figure 8.4 depicts the concept of $N T=4$. A short training block is sent prior to the sub-block in TMT-fashion, while pilot symbols are also inserted in the sub-blocks along with the data symbols in FMT-fashion.

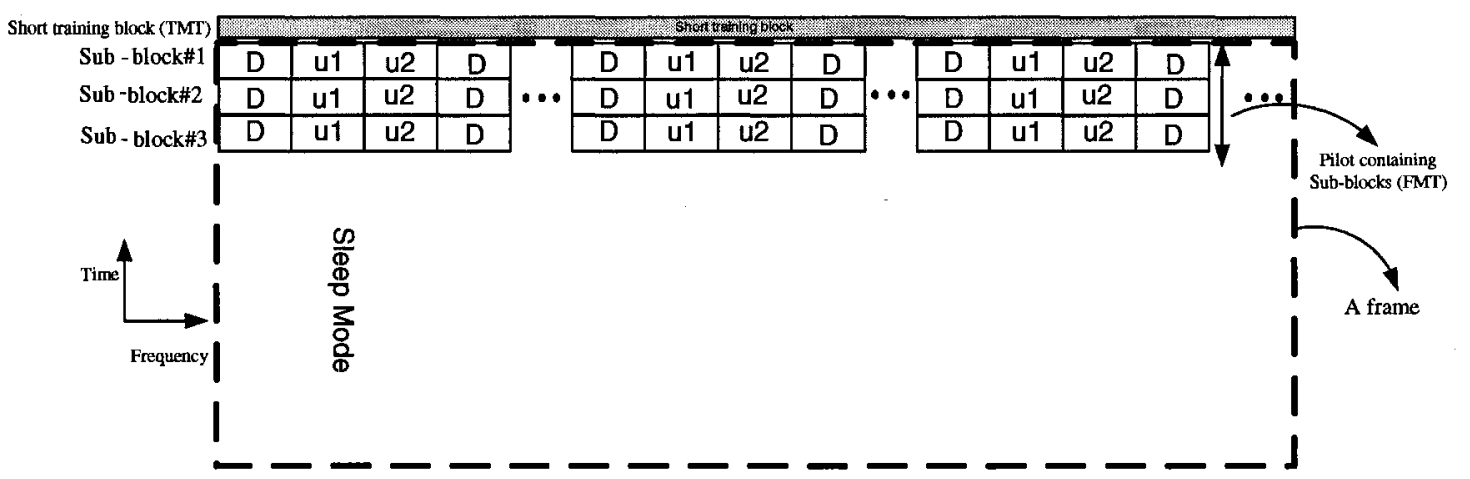

Fig. 8.4. Pilot distribution in a frame of B-IFDMA (concept of $N T=4$, a combination of TMT and FMT). 


\subsection{SD-IBDFE Performance Evaluation}

SD-IBDFE assisted non-adaptive SC-FDE B-IFDMA system is evaluated. SD-IBDFE uses the pilot-assisted estimated CFRs of the ICUs. The system parameters are summarized in Table 8.1.

Table 8.1. B-IFDMA parameters.

\begin{tabular}{|c|c|}
\hline Number of sub-carriers per block & 4 \\
\hline Number of SC-FDE sub-blocks in a block & 3 \\
\hline Used sub-carriers & $32 \times 4=128$ \\
\hline Number of sleep sub-blocks/frame & 9 \\
\hline Mobility in the channel & $50 \mathrm{~km} / \mathrm{hr}$ \\
\hline Inter-block spacing & 32 sub-carriers \\
\hline Number of sub-blocks/Code Frame $(F)$ & $K=2$ \\
\hline Number of ICUs & $P=4$ \\
\hline Number of Out of Cell users & $M=4$ \\
\hline Number of receiving antenna elements & 1 or 2 \\
\hline Number of pilots per block per user & $N T=1$ or 2 \\
\hline
\end{tabular}

The performance is evaluated with a block size of $4 \times 3$ (sub-carriers $\times$ DFT-pre-coded OFDM sub-blocks). We assume four antenna elements at the desired BS with two orthogonal ICUs and four overlapping OCIs at an average received power levels of $15 \mathrm{~dB}$ and 18.2dB below the ICUs. Either one or two FMT pilots per block per ICU are used, termed as F1-12 and F2-12 respectively. As the number of pilots per block is constrained by the block size, initial pilot-based estimates will either experience significant performance degradation and/or a large pilot overhead. In the simulations, the channel encoder is a memory 6 Convolutional Code (CC) with generator polynomials $(133,171)$ in octal form. In F1-12, no interpolation is possible, while in F2-12 a Linear Interpolator (LI) is used to get the estimates of the CFR at the remaining 2 frequencies per sub-block.

In Figure 8.5, the average FER performance with different pilot overheads using pilots for $\mathrm{CE}$, without using ICE, with $4 \times 3$ block size and 32 inter-block spacing is compared. A frequency spacing of 32 sub-carriers $=32 \times 39.0625=1.25 \mathrm{MHz}$ is on the same order of magnitude as the coherence BW of the C2 channel. It is observed that for PCSI, the performance of B-IFDMA is very good. However, if CE is taken into account, the 
performance of B-IFDMA severely degrades. F1-12 with $N T=2$ scheme with an overhead of $33.33 \%$, using SD-IBDFE, the performance degradation exceeds $8 \mathrm{~dB}$. With a greater pilot overhead of F1-12 with $N T=4$, the FER is improved by about $2 \mathrm{~dB}$. With two pilots per block F2-12 with $N T=4$, the SNR degradation gap reduces to 5.2dB. Results with noOCI case and lower average power OCIs (@-18.2dB) are also shown.

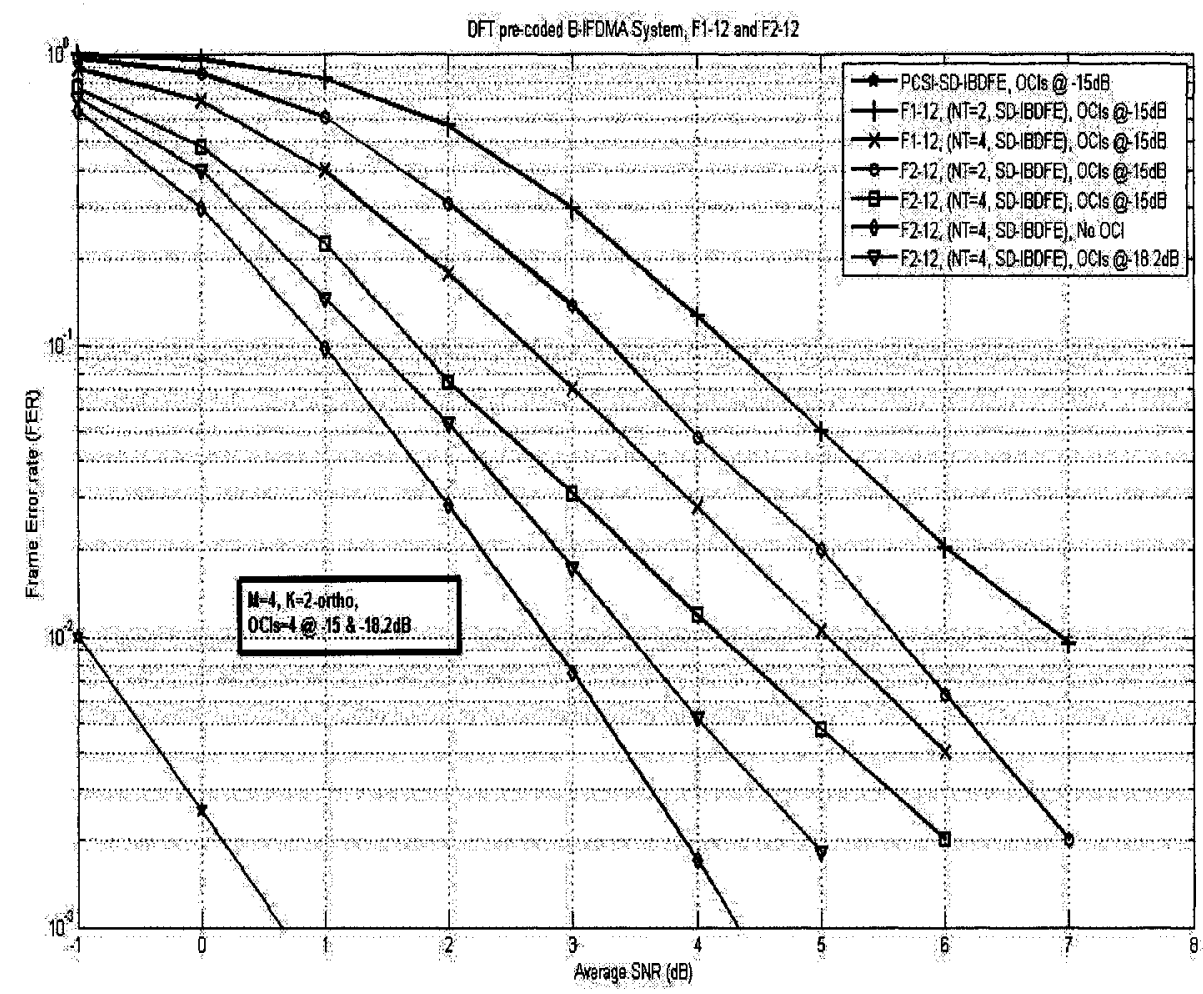

Fig. 8.5. Average FER-Comparison for B-IFDMA with $4 \times 3$ block size, CE using pilots and $2 \times 1 \mathrm{D}$ interpolation, SD-IBDFE with no DFICE, $M=4, N T=2$ and 4.

\subsection{DFICE-Smoothing via Averaging}

We invoke (6.1) to obtain the hard decision-based $\left(\tilde{S}_{k}^{j}[n]\right)$ decision-directed raw CFR estimates for the whole block. Smoothing of the estimates over four frequencies is performed in each sub-block by means of simple averaging over the four frequencies in each sub-block.

Figure 8.6 illustrates the comparison of the HD-DFICE scheme performance with the receiver having only SD-IBDFE and pilot-based CE. An improvement of $1 \mathrm{~dB}$ with F2-12 
with $N T=2$ is achievable with the employment of the HD-DFICE. Similarly, with $N T=4$, a further improvement of around $0.8 \mathrm{~dB}$ is observed. Improvement with the lower relative average power OCIs $(-18.2 \mathrm{~dB})$ and with no-OCI case is $0.6 \mathrm{~dB}$. The smaller difference is due to the better initial pilot-assisted CFR estimates in case of lower power or no-OCI case.

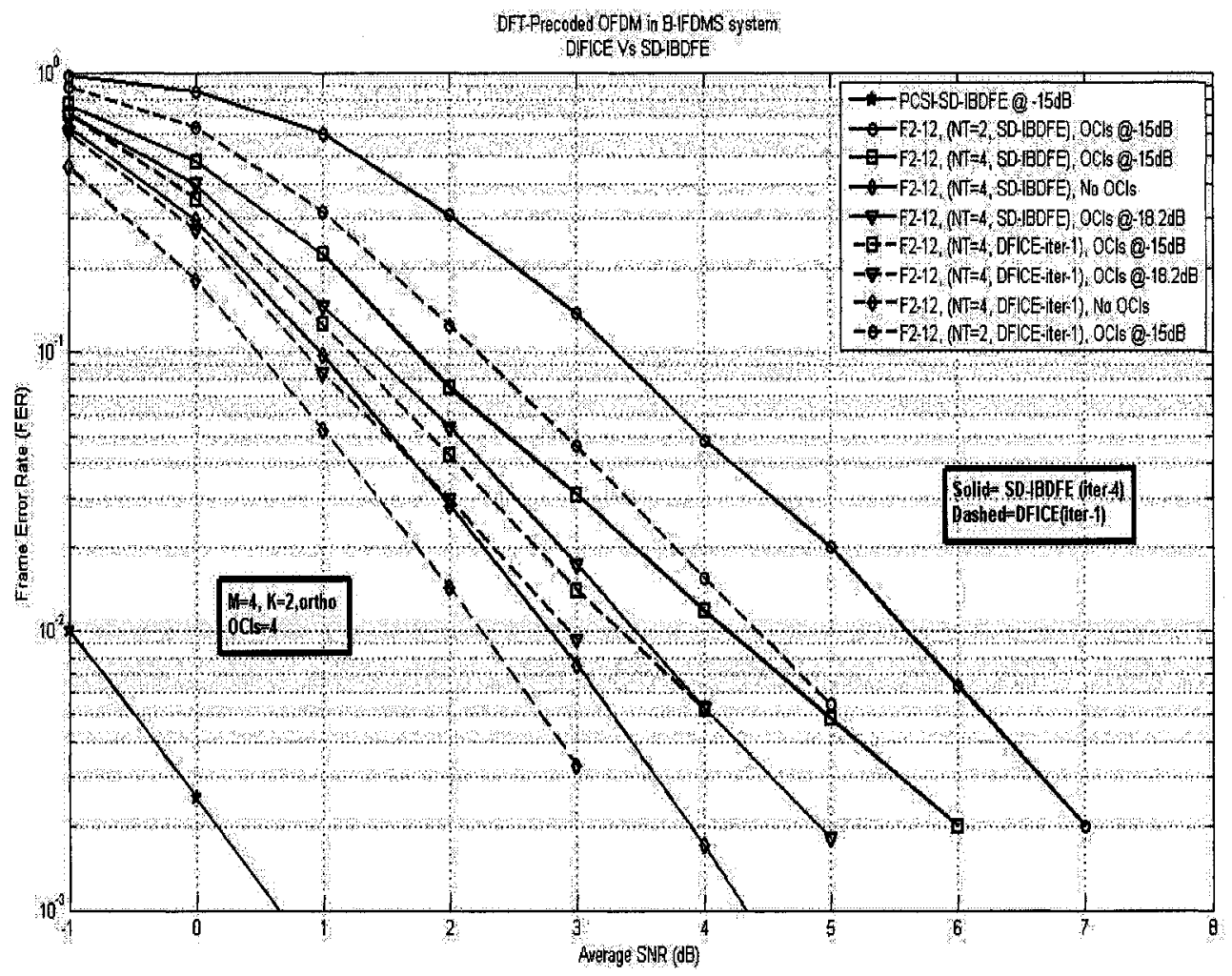

Fig. 8.6. Average FER-Comparison for B-IFDMA with $4 \times 3$ block size.

Although the HD-DFICE employs smoothing by averaging and achieves an improvement of about $1 \mathrm{~dB}$, but still B-IFDMA has a huge SNR degradation with estimated CFRs. The cause of the SNR degradation gap of SD-IBDFE is twofold: first, the poor initial channel estimates, since there is a lack of 2x1D Wiener interpolation, which is only possible in full BW case; second, in B-IFDMA, HD-DFICE there is a lack of smoothing filtration on the decision-based CFR estimates over the four frequencies in a sub-block. Moreover, there is degradation due to no OCI mitigation by LS-IS. The addition of LS-IS processing does not reduce SNR degradation for B-IFDMA, as there are only 3 sub-blocks per frame that can be used for time averaging. On the other hand, very good performance of the 
PCSI is a result of frequency diversity and the size of the codeword. The number of coded symbols per frame in different systems is given as:

1- Full BW is $1024 \times 12$ (sub-carriers $x$ sub-block) $=12288$ coded symbols per frame.

2- B-IFDMA with sleep mode is $4 \times 32 \times 3$ (sub-carriers $\times$ chunks $\times$ sub-block) $=384$ coded symbols per frame.

B-IFDMA with sleep mode has 32 times shorter code block compared to the FBW case. To confirm this reasoning, the case of B-IFDMA with no sleep mode is simulated, having a slightly larger code block length compared to the sleep mode case. The corresponding number of symbols per frame will be $4 \times 32 \times 12=1536$ coded symbols per frame.

B-IFDMA with no sleep mode has 4 times larger frame length compared to the case of BIFDMA with sleep mode. As illustrated in Figure 8.7, the PCSI curve with B-IFDMA with no sleep mode is between the other two cases. Hence, due to the short frame lengths, the PCSI FER of B-IFDMA performs much better than the FBW scenario. It should be noted that short frame length results in less FER, but the difference in the BER will not be considerable.

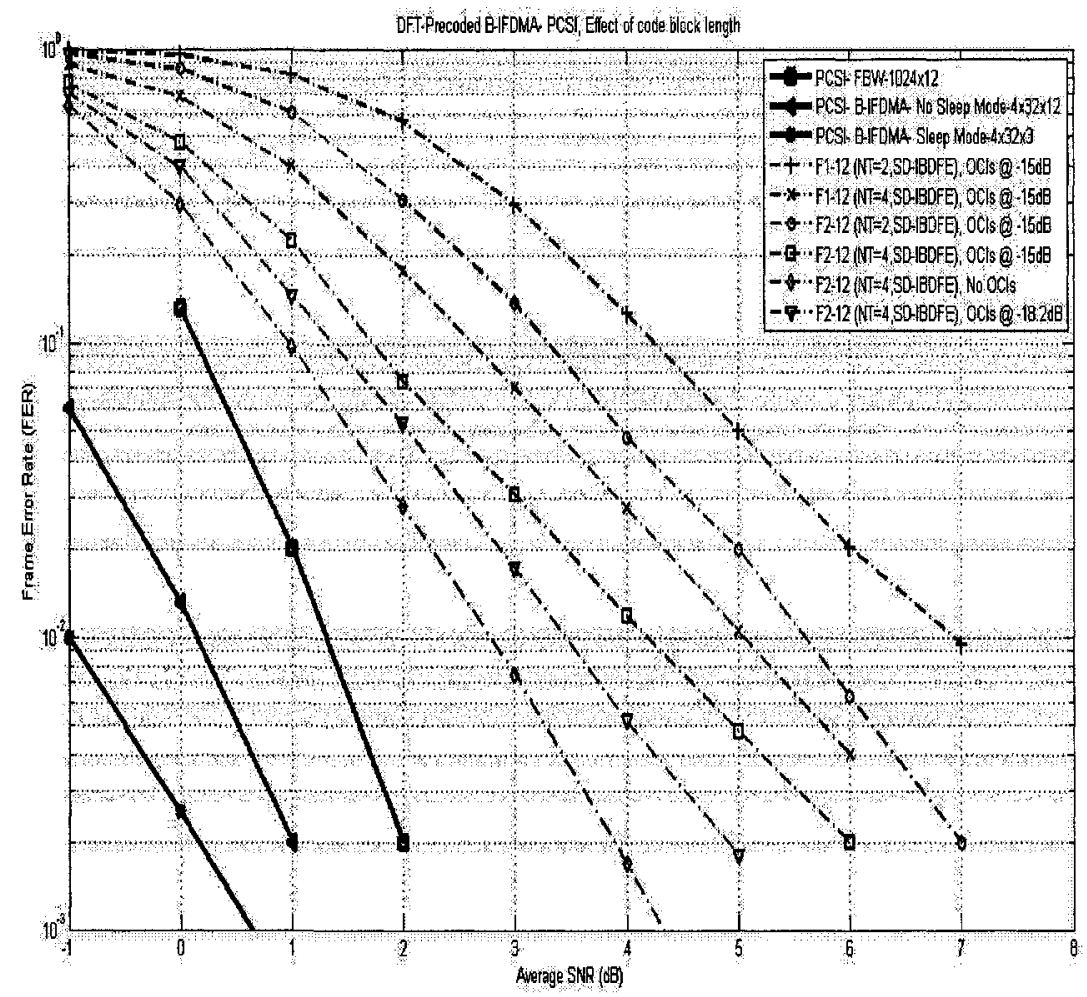

Fig. 8.7. Effect of code block size on average FER. 


\subsubsection{Results with $N T=1$}

Higher values of $N T$ average out the noise and the unknown OCIs and provide the SDIBDFE a better set of ICUs' CFR estimates. Figures 8.5 and 8.6 show results with $N T=2$ or more. More $N T$ costs a proportional pilot overhead. In this section we simulate the HD-DFICE performance with $N T=1$. Figure 8.8 shows the results with $M=4$ (antenna elements), $K=2$ (orthogonal ICUs), $N T=1$, with $P=4$ (OCIs) and $P=0$ respectively. Results with $N T=2$ or more from Figure 8.6 are also shown (dashed lines) for comparison purposes.

We concluded in Chapter 6 that worse initial CFR estimates because of the lesser NT could be covered by utilizing more DFICE iterations $\left(I_{\mathrm{ICE}}\right)$ with FBW. In contrast, with B-IFDMA although more $I_{\mathrm{ICE}}$ improves the FER (absolute $\mathrm{SNR}=5 \mathrm{~dB}$ with $I_{\mathrm{ICE}}=3, N T=1$ ) it could not give performance equivalent to that provided by the $N T=2$ case (absolute $\mathrm{SNR}=4.5 \mathrm{~dB}$ with $I_{\mathrm{ICE}}=1, N T=2$ ). This is mainly due to the absence of LS-IS processing and smoothing in HD-DFICE in B-IFDMA.

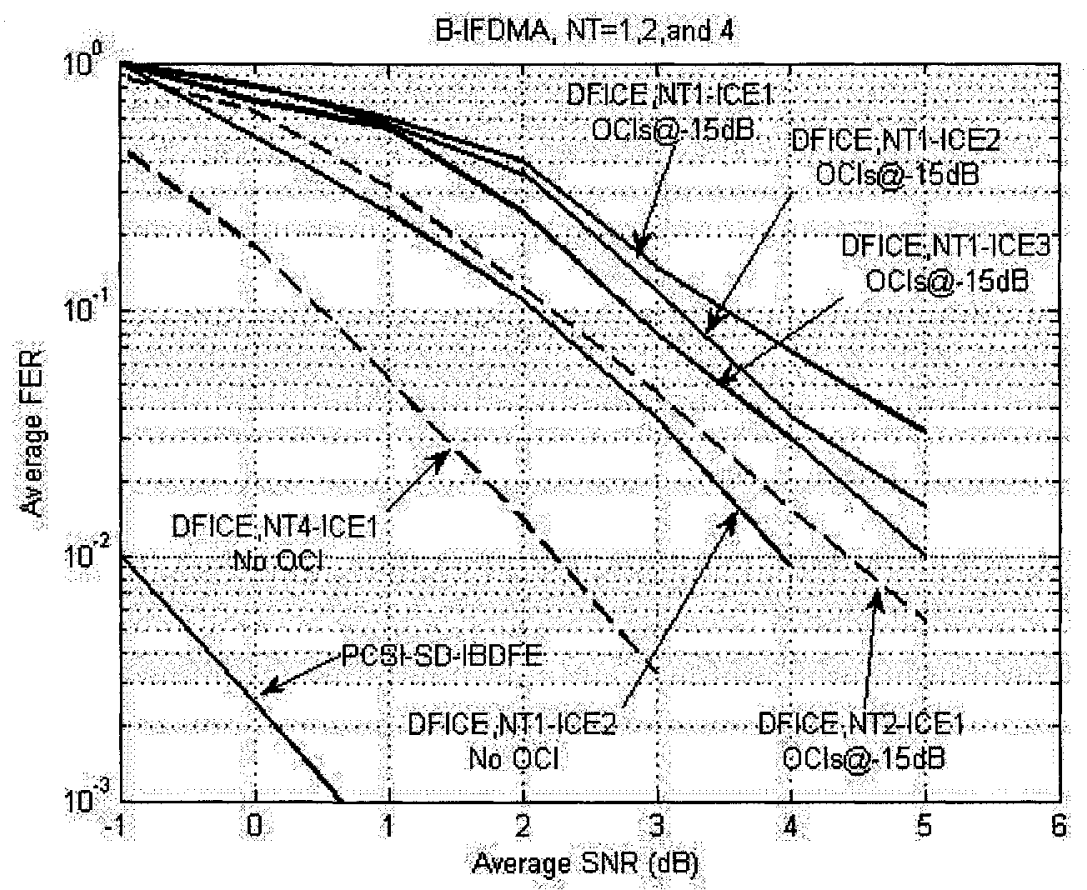

Fig. 8.8. Average FER results with B-IFDMA - with or without OCIs @-15dB $(N T=1,2$ and 4). 
Some more results that are not shown graphically in the figures are given in Table 8.2 and Table 8.3 in Section 8.8 where we present the concluding remarks and discussions on the CE performance of two chunk-based systems. For example a block size of $8 \times 6$ is also simulated. There are 4 pilots per block per user; the corresponding total pilot overhead is 16.6\%. Figures 8.9 and 8.10 depict results with B-IFDMA of $8 \times 6$ block size, with only PCSI and with CE respectively. Comments on these results are given in the Section 8.8.

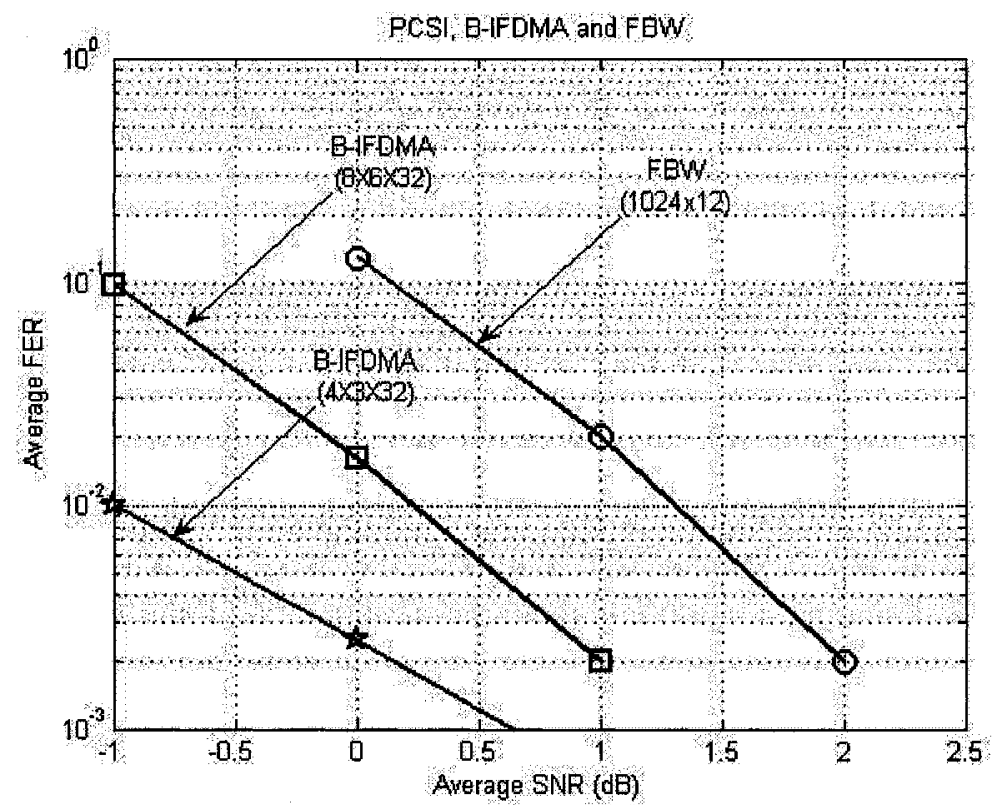

Fig. 8.9. Average FER results with B-IFDMA - with or without OCIs @-15dB. 


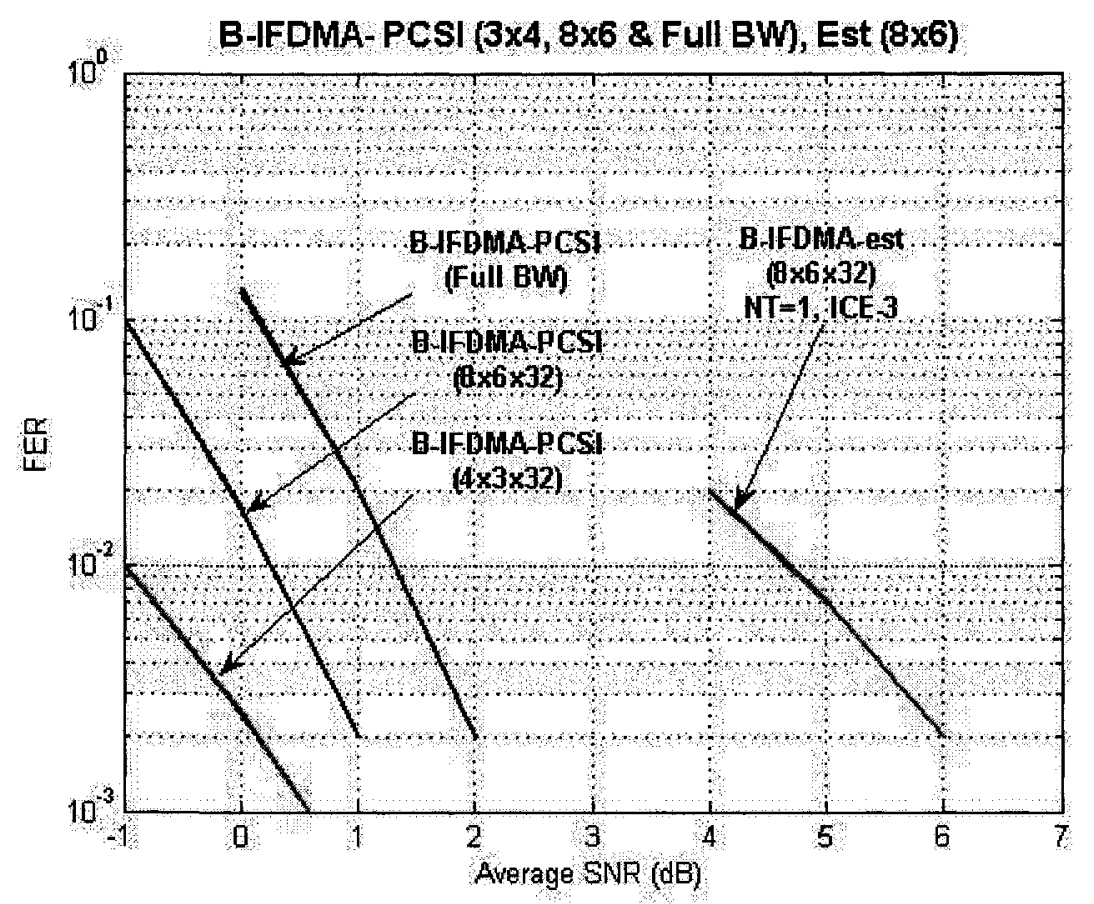

Fig. 8.10. Average FER results with B-IFDMA - with or without OCIs @-15dB $(N T=1)$.

\subsection{CE over Multiple Frames}

With FBW system, we have shown in Chapter 6 that some performance gain is achievable with CE over multiple frames. In a similar way, in B-IFDMA we denote the total number of consecutive frames as $F$ r. Figure 8.11 shows a scenario with $F r=2$. 


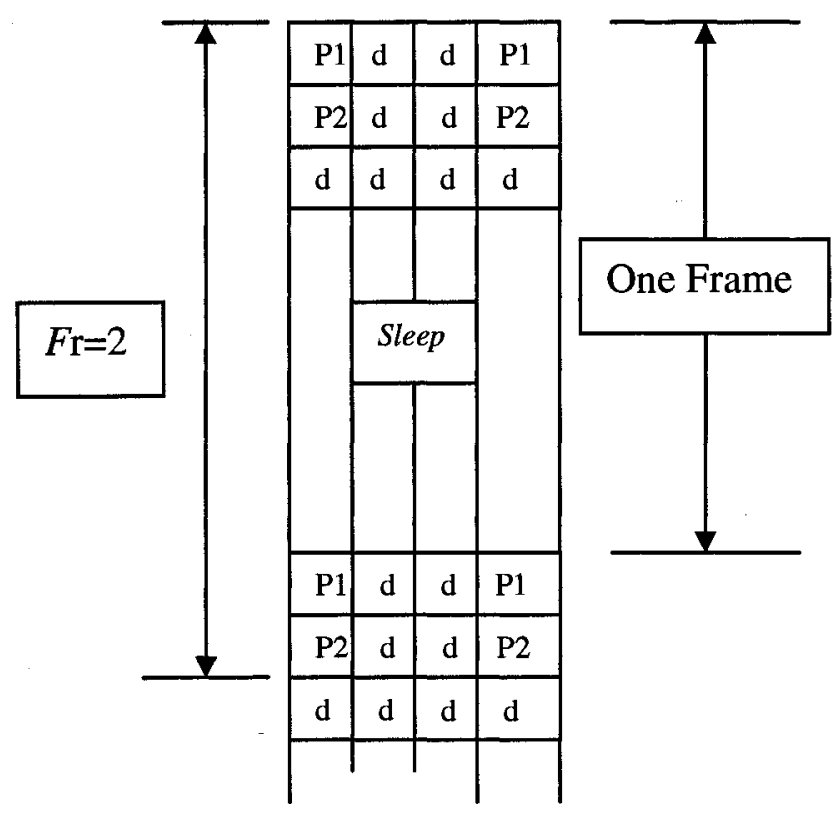

Fig. 8.11. Channel estimation over multiple frames in B-IFDMA.

There is no difference in the frequency axis $\mathrm{CE}$, while with $\mathrm{Fr}=2$ as shown in Figure 8.11 , in time direction the $\mathrm{CE}$ is carried out with linear interpolation between the first and $13^{\text {th }}$ sub-block. It should be noted that similar to FMW system each frame consists of one code block. About $1 \mathrm{~dB}$ improvement with $F \mathrm{r}=2$ over $F \mathrm{r}=1$ is achieved. Results are given in Table 8.2, in Section 8.8.

\subsection{L-FDMA}

It is evident from above results that B-IFDMA CE performance is much worse than the FBW system. It would be fairer to compare the performance of the equal block length systems. One of the candidates is a partial-band system or so-called Local Frequency Division Multiple Access (L-FDMA) system. The concept of L-FDMA is exactly as BIFDMA, but instead of $324 \times 3$ blocks per frame, at regular 32 sub-carrier intervals, there is only one $32 \times 12$ block per frame. Just as for B-IFDMA, each user has $32 \times 12=384$ QPSK coded symbols per frame. However, the B-IFDMA spectrum spans $128 \times 39.0625$ $\mathrm{KHz}=5 \mathrm{MHz}$, while the L-FDMA signal spans only $32 \times 39.0625 \mathrm{KHz}=1.25 \mathrm{MHz}$. LFDMA frame structure is depicted in Figure 8.12. 


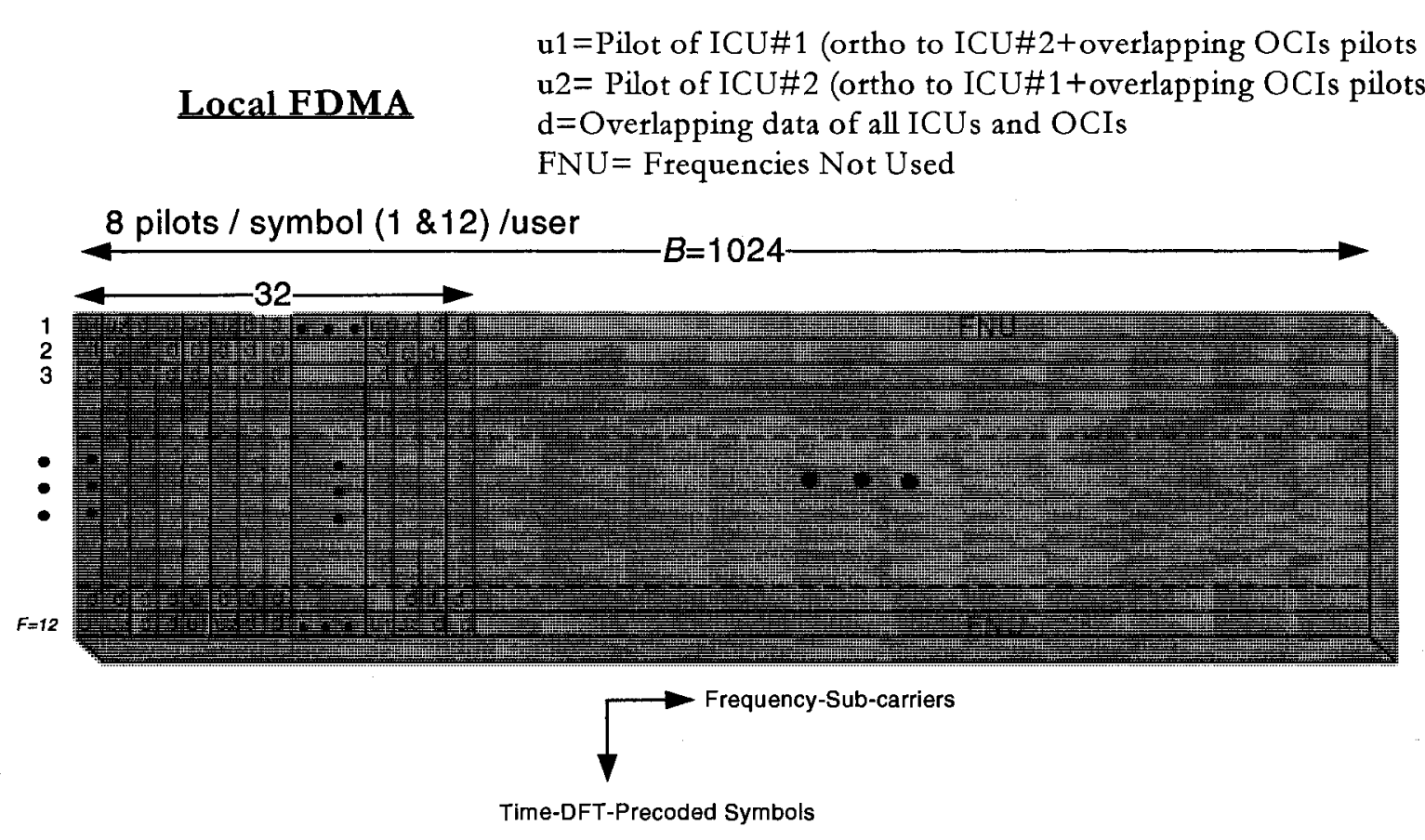

Fig. 8.12. Pilot arrangement for an L-FDMA chunk-based system.

\subsubsection{Pilot Arrangements and DFICE Performance}

The pilot arrangement or spreading in L-FDMA is performed in a similar way as was done in the FBW system described in Chapter 6. Pilots are multiplexed in FMT manner; 8 pilots are spread in the first and the $12^{\text {th }}$ block and a block contains 32 frequencies. ICUs' pilots are kept orthogonal while the OCIs' pilots overlap the ICUs' pilots. All twelve blocks are used (i.e. no sleep mode) to transmit data. Interpolation in both time and frequency axes is possible with L-FDMA. Moreover, in contrast to the B-IFDMA, LFDMA allows smoothing filtration in the DFICE process. We expect the L-FDMA signal to have worse performance than B-IFDMA with PCSI, since L-FDMA benefits from much less frequency diversity, but its CE SNR degradation from the PCSI may be less than that of B-IFDMA.

\subsubsection{Simulation Results}

In this section we evaluate the average FER performance of the L-FDMA system. Figure 8.13 illustrates the L-FDMA FER performance curves with PCSI and with FMT based LS-IS assisted HD-DFICE. In comparison to Figure 8.6, it is observed that with $M=4$, 
$K=2$ and $P=4 @-15 \mathrm{~dB}$ L-FDMA performs around $2.5 \mathrm{~dB}$ worse than B-IFDMA with PCSI. This is mainly due to the lack of frequency diversity in L-FDMA. But the SNR degradation with L-FDMA due to the CE with $N T=1$ (overhead=8.3\%) is around $4.5 \mathrm{~dB}$ which is $1 \mathrm{~dB}$ less than that of B-IFDMA whose overhead=33.33\%. As far as the absolute SNR with CE is concerned L-FDMA is still worse than B-IFDMA.

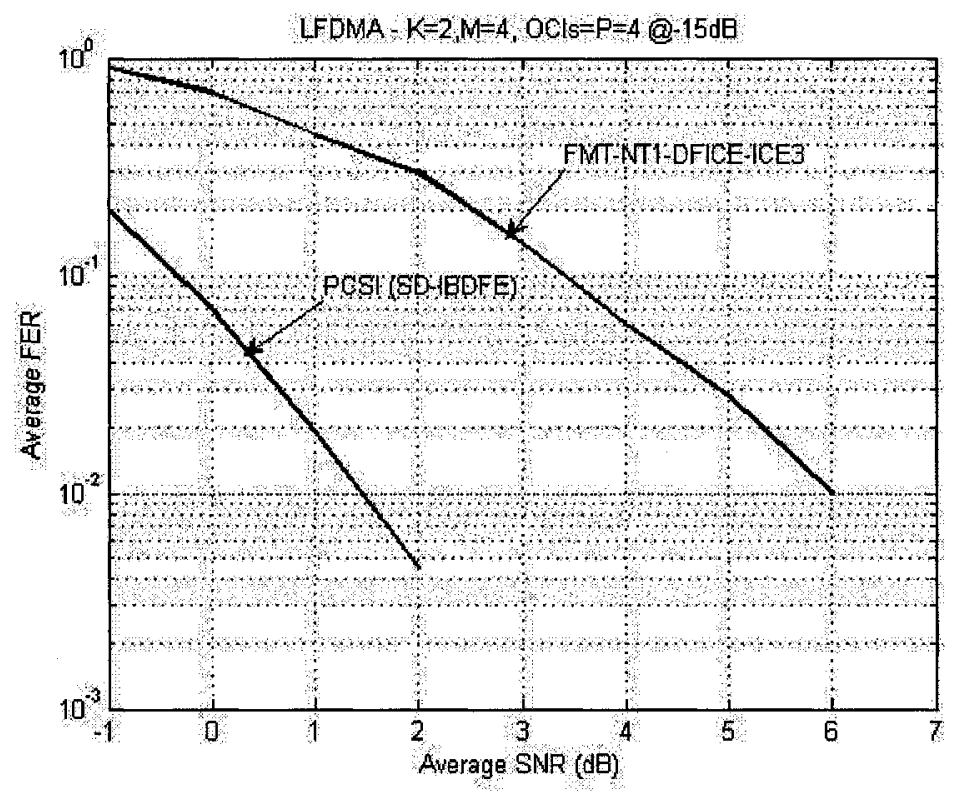

Fig. 8.13. Average FER performance, L-FDMA, $M=4, K=2, P=4$.

One interesting aspect with L-FDMA and B-IFDMA could be to observe their performances at lower instantaneous baud rates. Figure 8.14 illustrates such comparison between B-IFDMA and L-FDMA with the following simulation assumptions:

- Single Input Single Output (SISO) link i.e. $M=K=1$,

- Number of training blocks, $N T=1$,

- Number of OCIs, $P=0$,

- C2-channel with mobility of $50 \mathrm{~km} / \mathrm{hr}$.

- B-IFDMA with $4 \times 3$ blocks, 2 pilot per block (16.67\% overhead).

- L-FDMA with 4 (2 pilots per pilot carrying symbol) pilots per chunk $(4.16 \%$ overhead).

Three different instantaneous data symbol rates are simulated given as: 


\section{Case-I (Labeled as -32)}

- 1.25 Mbaud instantaneous data symbol rate i.e. 384 coded data symbols per frame in 32 chunks for B-IFDMA and 4 chunks for L-FDMA.

\section{Case-II (Labeled as -64)}

- $2.50 \mathrm{Mbaud}$ instantaneous data symbol rate i.e. 768 coded data symbols per frame in 64 chunks for B-IFDMA and 8 chunks for L-FDMA.

\section{Case-III (Labeled as -128)}

- 5.0 Mbaud instantaneous data symbol rate i.e. 1536 coded data symbols per frame in 128 chunks for B-IFDMA and 16 chunks for L-FDMA.

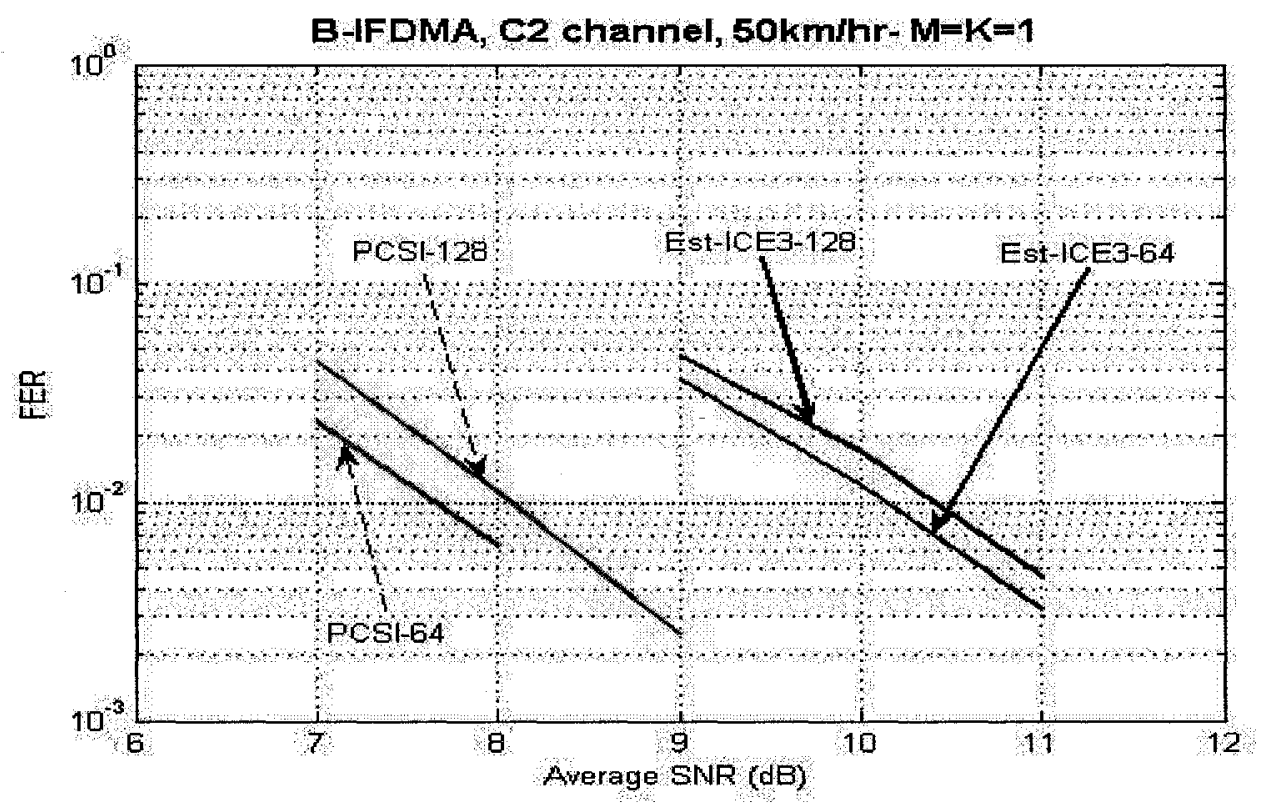

a) 


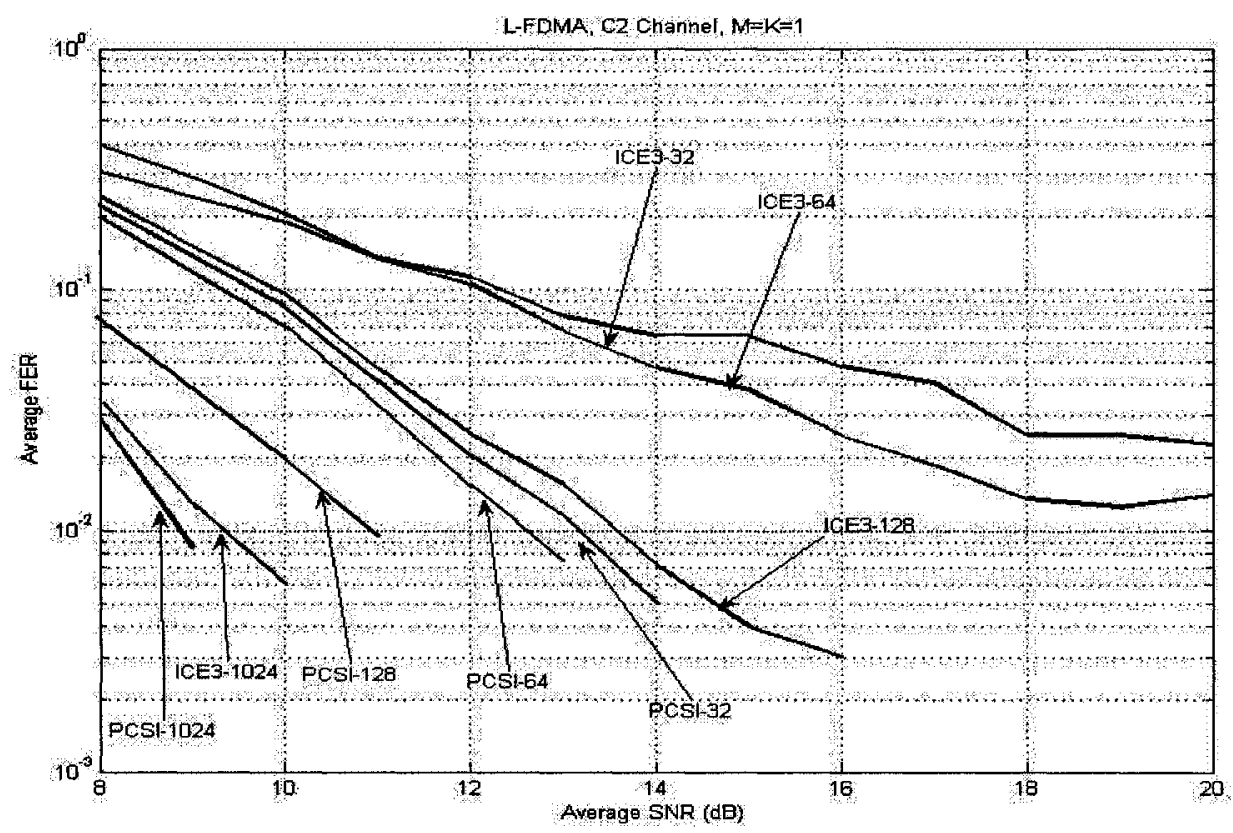

b)

Fig. 8.14. Average FER comparison between B-IFDMA-64, B-IFDMA-128 and LFDMA-32, L-FDMA-64, L-FDMA-128, $M=K=N T=1$, a) B-IFDMA b) L-FDMA.

Figure 8.14a shows the simulation results of B-IFDMA while Figure 8.14b illustrates the L-FDMA results with three different instantaneous baud rates. The performance of PCSI with B-IFDMA-128 (FER=0.02 @ 8.1dB) is better than the L-FDMA-128 (FER=0.02 @ 10.9dB). We get an error floor with CE in case of L-FDMA-32 and L-FDMA-64. With L-FDMA the performance improves with chunk size from $32 \times 12$ to $64 \times 12$ and then to $128 \times 12$ but the FER performance of B-IFDMA-128 never crosses (always better than) the L-FDMA-128. Hence we conclude that B-IFDMA offers superior performance compared to L-FDMA, at least for symbol rates at and below 5.0 Mbaud, with or without perfect CSI, although it has higher pilot overhead.

\subsection{Concluding Remarks}

Following are some concluding remarks about the B-IFDMA and L-FDMA simulation results. Also results at an average FER of $10^{-2}$ are summarized in tabular form in Table 8.2 and 8.3. 
Table 8.2. Summary of the comparison between B-IFDMA (4x3 and 8x6) and L-FDMA $M=4, K=2, P=4, N T=1$ and 2.

\begin{tabular}{|c|c|c|c|c|c|}
\hline & & \multirow{2}{*}{$\begin{array}{l}\text { SNR for } \\
\text { ideal CSI } \\
\text { (dB) for } 10^{-2} \\
\text { frame error } \\
\text { rate }\end{array}$} & \multicolumn{3}{|c|}{$\begin{array}{l}\text { SNR with channel estimation (dB) (SNR } \\
\text { degradation due to non-perfect CSI shown } \\
\text { in bold) }\end{array}$} \\
\hline & & & $\begin{array}{l}\text { Non- } \\
\text { iterative } \\
\text { channel } \\
\text { estimation }\end{array}$ & $\begin{array}{l}\text { Iterative } \\
\text { channel } \\
\text { estimation }\end{array}$ & $\begin{array}{l}\text { Iterative } \\
\text { channel } \\
\text { estimation } \\
\text { plus least } \\
\text { squares }\end{array}$ \\
\hline \multirow{4}{*}{$\begin{array}{l}\text { B-IFDMA with } \\
4 \times 3 \text { blocks, } 32 \\
\text { subcarrier spacing } \\
\text { between blocks. } \\
2 \text { pilots per block } \\
\text { per user ( } 33.3 \% \\
\text { total pilot } \\
\text { overhead for } 2 \text { in- } \\
\text { cell users). }\end{array}$} & $\begin{array}{l}\text { No OCIs } \\
\text { NT=1, } E=1\end{array}$ & -1.5 & $5.8(7.3)$ & $3.9(5.4)$ & $3.9(5.4)$ \\
\hline & $\begin{array}{l}\text { No OCIs } \\
\underline{N T=2, F}=1\end{array}$ & -1.5 & $4.0(5.5)$ & $2.7(4.2)$ & $2.7(4.2)$ \\
\hline & $\begin{array}{l}\frac{4 \text { OCIs } \text {, each at - }}{15 \mathrm{~dB}} \\
N T=1, F_{\mathrm{r}}=1\end{array}$ & -1.0 & $7.5(8.5)$ & $5.0(6.0)$ & $5.0(6.0)$ \\
\hline & $\begin{array}{l}\frac{4 \text { OCIs }}{15 \mathrm{~dB}} \text { each at }- \\
N T=2, F_{\mathrm{r}}=1\end{array}$ & -1.0 & $5.6(6.6)$ & $4.5(5.5)$ & $4.5(5.5)$ \\
\hline $\begin{array}{l}\text { B-IFDMA with } \\
\text { 4x3 blocks, } 32 \\
\text { subcarrier spacing } \\
\text { between blocks. } \\
2 \text { pilots per block } \\
\text { per user ( } 33.3 \% \\
\text { total pilot } \\
\text { overhead for } 2 \text { in- } \\
\text { cell users). }\end{array}$ & $\begin{array}{l}\frac{4 O C I s}{15 \mathrm{~dB}} \text { each at - } \\
N T=1, F_{\mathrm{r}}=2\end{array}$ & & - & $4.0(5.0)$ & \\
\hline $\begin{array}{l}\text { L-FDMA using } 4 \\
\text { adjacent chunks. } \\
8 \text { pilots per user } \\
\text { per chunk ( } 8.3 \% \\
\text { total pilot } \\
\text { overhead for } 2 \text { in- } \\
\text { cell users) }\end{array}$ & $\begin{array}{l}\frac{4 \text { OCIs }}{15 \mathrm{~dB}} \text { each at - } \\
N T=1, F_{\mathrm{r}}=1\end{array}$ & 1.5 & & $6.0(4.5)$ & \\
\hline $\begin{array}{l}\text { B-IFDMA with } \\
\text { 8x6 blocks, } 32 \\
\text { subcarrier spacing } \\
\text { between blocks. } \\
\text { ( } 16.6 \% \text { total pilot } \\
\text { overhead for } 2 \text { in- } \\
\text { cell users). }\end{array}$ & $\begin{array}{l}\frac{4 \text { OCIs }}{15 \mathrm{~dB}} \text { each at - } \\
N T=1, F_{\mathrm{r}}=1\end{array}$ & 0.3 & $6.8(6.5)$ & $5.4(5.1)$ & $4.7(\mathbf{4 . 4})$ \\
\hline
\end{tabular}


Table 8.3. Summary of the comparison between B-IFDMA (4x3) and L-FDMA

$$
M=K=N T=1 \text {. }
$$

\begin{tabular}{|c|c|c|c|}
\hline \multirow{2}{*}{\multicolumn{2}{|c|}{ Pilot schemes }} & \multicolumn{2}{|c|}{$\begin{array}{l}\text { SNR with channel estimation (dB) (SNR } \\
\text { degradation relative to perfect CSI shown in } \\
\text { bold) }\end{array}$} \\
\hline & & $\begin{array}{l}\text { SNR for perfect CSI } \\
\text { (dB) for } 10^{-2} \text { frame } \\
\text { error rate }\end{array}$ & $\begin{array}{l}\text { Iterative channel est. } \\
\text { with decoding in } \\
\text { iteration loop }\end{array}$ \\
\hline \multirow{2}{*}{$\begin{array}{l}768 \text { coded data } \\
\text { symbols per frame } \\
\text { in } 64 \text { chunks for B- } \\
\text { IFDMA and } 8 \\
\text { chunks for L-FDMA } \\
(2.50 \text { Mbaud } \\
\text { instantaneous data } \\
\text { symbol rate) }\end{array}$} & $\begin{array}{l}\text { B-IFDMA with } \mathbf{4 x 3} \\
\text { blocks, } 1 \text { pilot per } \\
\text { block }(8.3 \% \\
\text { overhead })\end{array}$ & 7.7 & $10.2(2.5)$ \\
\hline & $\begin{array}{l}\text { L-FDMA with } 4 \\
\text { pilots per chunk } \\
(4.1 \% \text { overhead })\end{array}$ & 12.5 & FER floor above $10^{-2}$ \\
\hline \multirow{2}{*}{$\begin{array}{l}\text { 1536 coded data } \\
\text { symbols per frame } \\
\text { in } 128 \text { chunks for B- } \\
\text { IFDMA and } 16 \\
\text { chunks for L-FDMA } \\
\text { ( } 5.0 \text { Mbaud } \\
\text { instantaneous data } \\
\text { symbol rate) }\end{array}$} & $\begin{array}{l}\text { B-IFDMA with } \mathbf{4 x 3} \\
\text { blocks, } 1 \text { pilot per } \\
\text { block }(8.3 \% \\
\text { overhead })\end{array}$ & 8.1 & $10.4(2.3)$ \\
\hline & $\begin{array}{l}\text { L-FDMA with } 4 \\
\text { pilots per chunk } \\
(4.1 \% \text { overhead })\end{array}$ & 10.9 & $13.6(2.7)$ \\
\hline
\end{tabular}

\section{B-IFDMA:}

- B-IFDMA offers superior performance compared to L-FDMA, at least for rates at and below 5.0 Mbaud, with or without perfect CSI, although it has higher pilot overhead.

- With B-IFDMA doubling pilot overhead by changing $N T$ from 1 to 2 improves initial pilot-generated CFR estimates by almost $2 \mathrm{~dB}$ for noniterative $\mathrm{CE}$, and by 0.5 to $1.2 \mathrm{~dB}$ for DFICE.

- With B-IFDMA the presence of 4-15 dB Out of Cell Interferers (OCIs) degrades SNR for PCSI by only $0.5 \mathrm{~dB}$ (from $-1.5 \mathrm{~dB}$ to $-1.0 \mathrm{~dB}$ ), but degrades SNR with CE by several dBs.

- DFICE via averaging reduces SNR degradation significantly in the presence of OCIs. 
- Addition of LS processing does not reduce SNR degradation for B-IFDMA with a block size of $4 \times 3$, probably since there are fewer (only 3) DFTprecoded OFDM symbols used per frame that can be used for LS averaging.

- The 8x6-BIFDMA has 1.3dB higher required SNR for PCSI (which is due to its slightly lower frequency diversity), its SNR degradation with DFICE and LS processing is $1.6 \mathrm{~dB}$ less than that for the $4 \times 3$ case. One of the reasons for this is the use of 4 pilots instead of 2 in $8 \times 6$ case, which allows better interpolation in the time direction. Moreover, 8x6-BIFDMA performs better because of the effectiveness of the LS processing which provides a further gain of $0.7 \mathrm{~dB}$.

\section{L-FDMA:}

- New comparisons of B-IFDMA with equivalent-rate L-FDMA are conducted.

- 1.25 Mbaud L-FDMA suffers $2.5 \mathrm{~dB}$ loss relative to B-IFDMA for perfect CSI.

- In the presence of 4 OCIs $(P=4)$, L-FDMA has $4.5 \mathrm{~dB}$ loss due to non-perfect CSI, while B-IFDMA has a corresponding $6.0 \mathrm{~dB}$ loss.

- As a result, 1.25 Mbaud L-FDMA with DFICE has only a $1 \mathrm{~dB}$ loss compared to 1.25 Mbaud B-IFDMA.

- The pilot overhead for L-FDMA is much less than that of B-IFDMA.

- Low bit rate L-FDMA has worse performance than B-IFDMA for perfect CSI, but its SNR degradation due to CE with non-perfect CSI is somewhat less.

- In the case of B-IFDMA and IFDMA, channel frequency correlation cannot be effectively exploited to interpolate to non-pilot frequencies and to smooth noise.

\subsection{Summary}

In this chapter, we evaluated the CE performance in a multi-user chunk-based B-IFDMA system. It is observed that due to frequency diversity and shorter frame size we get better FER performance with PCSI while on the other hand due to the lack of the smoothing over four frequency sub-blocks and 2x1D Wiener interpolation, poor multi-user channel 
estimates are obtained. This causes a huge increase in the SNR degradation gap between the PCSI and the B-IFDMA CE curves. HD-DFICE improves the SNR degradation by an amount of 0.6-1 dB. L-FDMA system with the same instantaneous baud rates is also simulated. L-FDMA suffers worse CE performance due to the lack of frequency diversity. Overall the B-IFDMA offers superior performance compared to L-FDMA, at least for rates at and below 5.0 Mbaud. Hence the use of such chunk-based systems should be directed by the compromise between the gains from frequency diversity and power saving versus the worse CE performance. 


\section{Chapter 9}

\section{Conclusions and Future Work}

\subsection{Introduction}

In this chapter, conclusions and possible future work towards the suppression of multiuser interference are briefly described.

\subsection{Conclusions}

In this thesis we addressed the issue of multi-user channel estimation problem in an interference-prone multi-cellular uplink SC-FDE environment. The problem of multi-user interference is too large in scope to be handled with a single algorithm. A practical approach is to use a combination of several techniques to achieve a reasonable $\mathrm{CE}$ results. In this thesis we apply several techniques to improve the multi-user channel estimates. Following are few conclusions:

- With interference management techniques, it is shown that reasonably good initial channel estimates can be obtained by orthogonal pilot multiplexing either in time or frequency among ICUs.

- Separate time-frequency resources can be reserved for interfering OCIs to achieve better in-cell CE performance. But as the number of OCIs' streams increases, it is not possible to mitigate them with spatial signal processing; instead, it is more practical to treat OCIs as additive noise.

- Iterative equalization based on soft symbol estimates (SD-IBDFE) shows superior performance at higher SNRs compared with the LE and helps in improving the channel estimates in iterative CE process. This gives a notion that with improved channel estimates via any decision-directed approach, the proposed SD-IBDFE has ability to further suppress the interference. The block-based SD-IBDFE assists to remove ISI and suppresses the co-channel interference. As the feedback 
coefficients update involves soft decisions, the possibility of error propagation at higher SNR is reduced. As both feed forward and feedback filters are employed with efficient FFT implementation, the processing complexity is much lower compared to the DFEs based on the time domain feedback processing, especially in the case of larger channel memory.

- A threshold-based DFICE is proposed. Multi-user decision-directed iterative CE needs efficient in-cell and out of cell interference suppression from the received composite signal. It was found that this suppression can be achieved with the help of the proposed SD-IBDFE, PIC, LS adaptation, utilizing OCIs' sub-space and CE over multiple frames.

- With turbo equalization, an EM algorithm-based SD-DFICE algorithm is developed to improve the estimation performance. The SD-DFICE algorithm could be considered as the "ultimate" equalization and $\mathrm{CE}$ technique developed and evaluated in the thesis. Referring to Figure 7.10, it exceeds the next-best (HDDFICE) performance by $\sim 1.5 \mathrm{~dB}$ and it comes within about $0.5 \mathrm{~dB}$ of TE with PCSI at low (0 to $0.5 \mathrm{~dB})$ SNRs per antenna.

- CE aspects of a new chunk-based system called B-IFDMA are explored and the CE techniques developed for the full bandwidth SC-FDE systems are applied to such systems. The use of such chunk-based systems should be directed by the compromise between the gains from frequency diversity and power saving versus the worse CE performance.

- It is also found (See Appendix B) that the effect of asynchronous low-power OCIs is not severe and can be neglected. Although necessary signal processing is needed if the BS experiences higher power levels of the asynchronous OCIs.

\subsection{Future Work}

\subsubsection{Simulation Environment}

One of the extension of our current work is to test the algorithms in an environment with correlated channels, log-normal shadowing effects, frequency offset and phase noise effects in both full bandwidth and chunk-based B-IFDMA systems. 


\subsubsection{Comparison of Proposed SS-IS and LS-IS with more Complex Optimization Algorithms}

More complex optimization algorithms to suppress the inter-user interference such as the Genetic Algorithm with turbo equalization can be investigated as a future work. It is considered that the genetic algorithm is complex, and that its stability and convergence highly depends on the process parameters [HK06]. Within WINNER, there is a simulation attempt to use the genetic algorithm to estimate the channel along with the turbo processing [Win03]. In those simulations no OCIs was considered. It will be worthwhile to compare such algorithms in the presence of inter-cell interference. Results in terms of SNR degradation should be compared with the proposed LS-adaptation based SD-DFICE results. The obvious advantage of the proposed schemes over the genetic algorithm is their lower complexity.

\subsubsection{Transmitter Adaptation}

We have used some advanced techniques implemented at the receiver to suppress the interference but we have not applied any transmitter adaptation or pre-coding technique to deal with the interference. Although they need channel information at the transmitter, exploration of such techniques with estimated channels fed back to the transmitter is one possible area of future research.

\subsubsection{Cross-Layer Efforts to Reduce Interference}

A marriage of the physical layer processing and the higher layer protocols is necessary to suppress the OCI effects. This could be done by putting more emphasis on interference avoidance via cell-sectoring, distributed antennas and slow frequency hopping like in GSM. Eventually the OCIs can be controlled by adjusting the traffic load in each interfering cell. Such cross-layer performance improvement efforts could be one of the extensions of this work. 


\subsubsection{Multi-User BERT Chart Analysis for TE}

Extrinsic Information Transfer (EXIT) chart and Bit Error Rate Transfer (BERT) chart are the analysis tools used to evaluate the iterative processing performance. The EXIT chart was introduced in [Bri01] and the BERT chart was introduced in [LS05 and references therein]. By using these analyses we may avoid the cumbersome and time consuming computer simulations which involve simulating the TE. Either of these tools has its own advantages and disadvantages. Exploring these tools, especially BERT chart analysis (due to its simplicity), and applying it in the multi-user scenario is one of the potential future works.

\subsubsection{BER Expression in the Presence of Interference}

Computing a closed form expression of BER or the outage probability for an interference-prone multi-user system is always a challenging topic. Researchers have been working on finding the exact or approximate (bounds) evaluation of the average BER in interference-prone flat or multi-path fading channels, limiting the number of interferers to one or two. Exploring such analytical expressions is also a potential future work as a continuation of the efforts in reducing the computer simulation burden and also verifying the simulation results and obtaining insight for examining the influence of various parameters on system performance. 


\section{Appendix A}

\section{A.1. Proposed Uplink Interference Simulation Scenario}

In this Appendix, a model for simulation of interference in wide area uplink multicellular system is suggested. Figure A.1 illustrates a system with three-sectored hexagonal cells with maximal radius $R$. The inner circles of the hexagonal cells with radius $0.7 R$ have three sectors named $\mathrm{A}_{1}, \mathrm{~A}_{2}$ and $\mathrm{A}_{3}$. Inner circles represent areas with frequency reuse 1 . The outer hexagonal areas labeled $B_{1}$, etc. have frequency reuse factor 3 , since they would otherwise be more vulnerable to interference from adjacent cells. The resources labeled $\mathrm{A}_{1}, \mathrm{~B}_{1}, \mathrm{~A}_{2}, \mathrm{~B}_{2}$ etc. are disjoint.

We assume that in a SDMA scenario, up to $K(K=1,2, \ldots)$ ICU terminals share the same transmission resources, and therefore interfere with one another in any given sector. Thus each user signal has up to $K$-1 equal-power in-cell interferers. For uplink transmission the average received power from ICUs is assumed to be equal, as a result of power control, to the power of a user on the edge of a cell or inner circle. ICUs in inner area (e.g. area $A_{1}$ shown in Figure A.1) also suffer out-of-cell interference from two nearby sectors (also labeled $\mathrm{A}_{1}$ ). The maximum number of OCIs from these two sectors is $2 \mathrm{~K}$. The minimum

distance of each of these OCIs to the BS is $\sqrt{3} R$. With WINNER urban coverage propagation exponent of 3.84 , the average received power of a minimum distance OCI relative to the average power of any ICU is given by:

$$
38.4\left[\log _{10}(0.7 / \sqrt{3})\right]=-15 \mathrm{~dB}
$$

The maximum OCI distance in this case is $2.7 R$. The corresponding relative average power is computed as:

$$
38.4\left[\log _{10}(0.7 / 2.7)\right]=-22.5 \mathrm{~dB}
$$




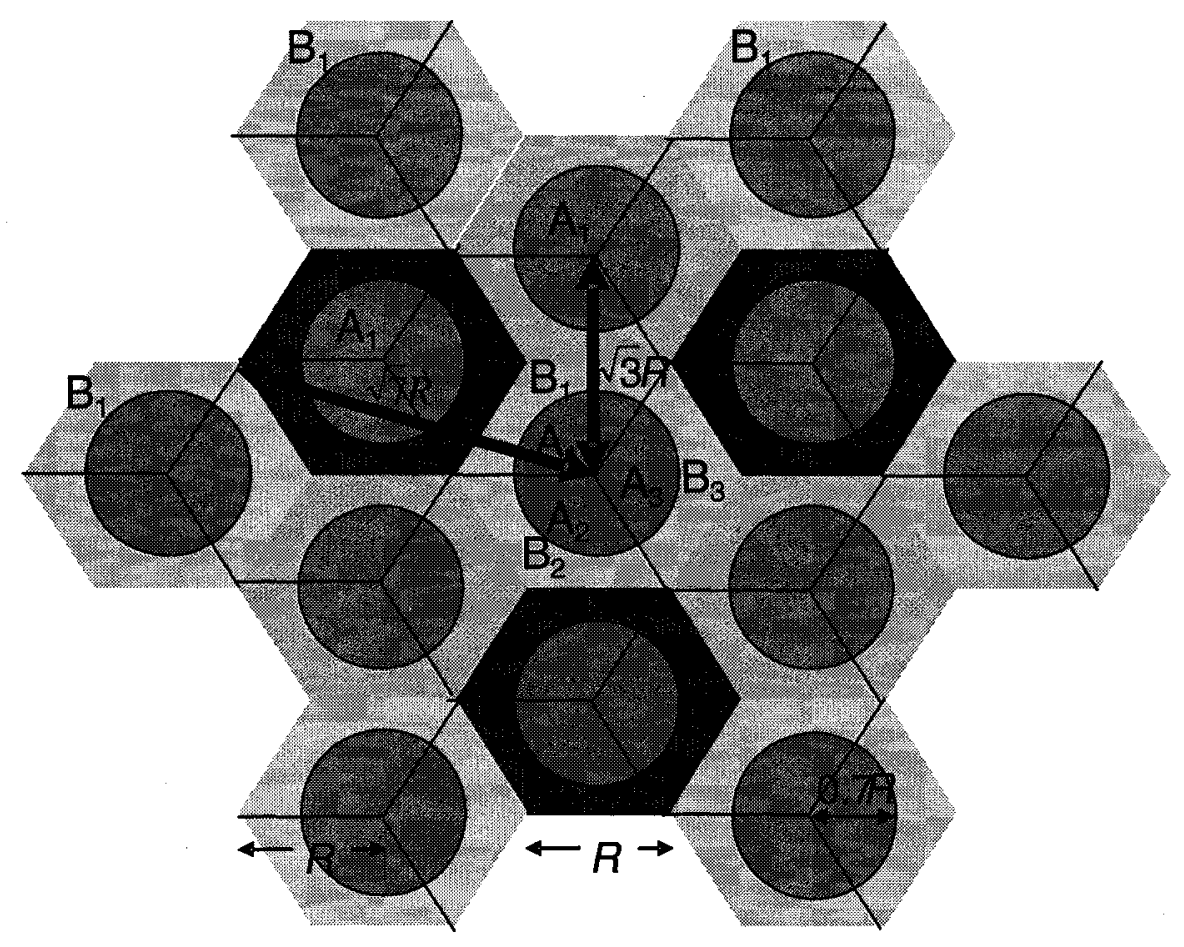

Fig. A.1. Cellular layout, showing frequency reuse zones

Similarly, for an ICU in region $B_{1}$, the nearest out-of-cell $B_{1}$ interferer appearing at the BS is at a distance of $\sqrt{7} \mathrm{R}$. The corresponding average power relative to an ICU signal is given by:

$$
38.4\left[\log _{10}(1 / \sqrt{7})\right]=-16 \mathrm{~dB}
$$

The maximum OCI distance for $\mathrm{B}_{1}$ is $4 R$ and similar to (A.2) the corresponding relative power is computed as:

$$
38.4\left[\log _{10}(1 / 4)\right]=-23 \mathrm{~dB}
$$

The maximum number of OCIs from the three nearest $B_{1}$ sectors is $3 K$.

\section{A.1.1. Suggested OCI Power Levels}

As a conclusion, with 3-sector hexagonal cells with center reuse-1 circles of radius $0.7 R$, and outer reuse- 3 areas with radius $R$, the ranges of maximum and minimum average $\mathrm{OCI}$ powers are the same, to within $1 \mathrm{~dB}$. For the purpose of link level simulations and 
evaluation of uplink SDMA channel estimation performance and pilot assignment, following interference scenarios can be suggested:

- Number of in-cell interferers (reflecting the degree of spatial multiplexing) $K$ 1 , all with average powers equal to that of the desired signal.

- Number of OCIs with average powers relative to the desired ICU uniformly spaced from $-15 \mathrm{~dB}$ to $-23 \mathrm{~dB}$. For example with 6 OCIs, the average powers would be: $-15 \mathrm{~dB},-16.6 \mathrm{~dB},-18.3 \mathrm{~dB},-19.8 \mathrm{~dB},-21.4 \mathrm{~dB}$ and $-23 \mathrm{~dB}$. We will be using OCIs' relative powers from this range like $-15 \mathrm{~dB},-16.6 \mathrm{~dB}$ and $-18.2 \mathrm{~dB}$.

- The channels between each transmitting antenna and each receiving BS antenna are independent, and they are scaled in relative power as indicated above. 


\section{Appendix B}

\section{B.1. Asynchronous Out of Cell Interferers (AOCIs)}

Signals from the ICUs are block-synchronized to the receiver, but as far as OCIs are concerned, this might not be the case. In multi-user SC-FDE systems, different propagation distances between ICUs and OCIs may cause asynchronous interference. This creates an additional source of Inter-Carrier Interference (ICI) when the delay differences between users are sizeable. The ICI contribution imposed on each sub-carrier can be viewed as an additional noise, which becomes near-Gaussian distributed as a consequence of the Central Limit Theorem (CLT) [HK06]. It would be worthwhile to explore the effects of the asynchronism of the OCIs on the overall system performance. A delayed OCI causing ICI is shown in Figure B.1.

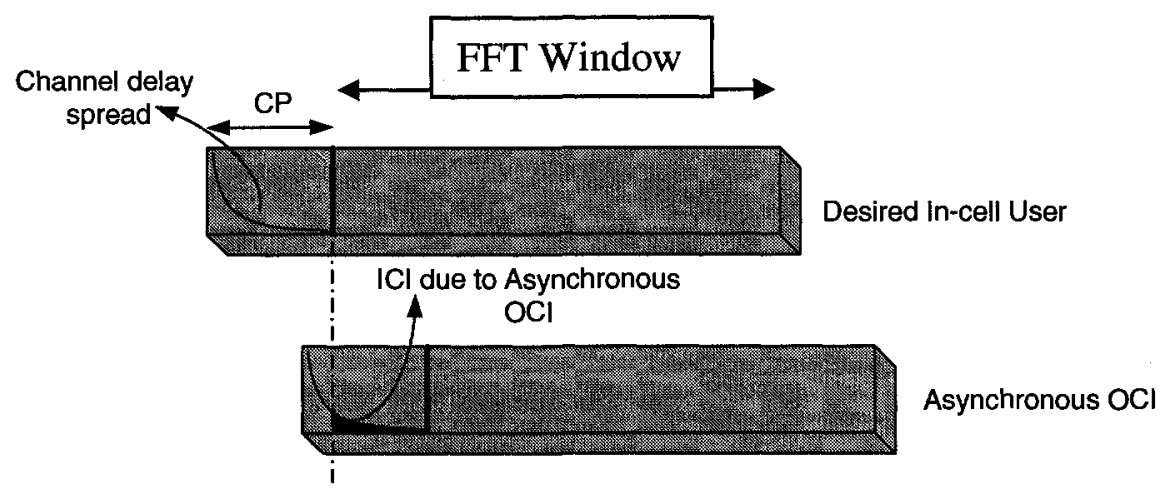

Fig. B.1. ICI caused by asynchronous OCI.

The CP enables the use of low complexity receiver spatial combining algorithms assuming that the signal is synchronized and the channel impulse response length is no longer than the CP length. When an interfering asynchronous OCI (AOCI) signal is present, the signal's CP may not align in time with that of the desired ICU's signal especially because of the differing propagation delays from the desired and interfering signals. Traditional frequency domain antenna combining techniques, such as linear MMSE combining, cannot perfectly suppress the interferer when its channel impulse response plus delay is greater than the CP length, since the DFT representation of the interferer, with uncoupled frequency components, no longer applies. As shown in Figure B.1, in a CP-based communication system, an asynchronous interferer is one whose CP is 
not aligned with the desired ICU's CP. This restricts the modeling of the asynchronous OCI in the frequency domain as the OCIs' channel on the tone multiplied by the data symbol. Eventually, the frequency domain weighing coefficients which are based on the assumption of cyclic convolution, are no longer capable of suppressing the asynchronous interferers. In fact, to completely suppress the AOCI using frequency domain cyclic convolution-based weights, all of the components of OCIs' impulse response must be suppressed on each tone [TV03].

\section{B.1.1. Effect of Asynchronism}

Before attempting to explore and apply the asynchronous interference suppressing techniques, let us look at the amount of ICI caused by the AOCIs in a multi-user SCEFDE system. It is predictable that very low relative power levels of the AOCIs might diminish the ICI part caused by the asynchronism. We consider a system with $K$ ICUs and $Z$ AOCIs with a receiver with $M$ antenna elements. We denote the delay of the AOCI's CP relative to the beginning of the desired in-cell signal's CP as $d_{O C I}$ and model it as $d_{O C I}$ zeros at the beginning of the IR for the channels from the interfering OCU. The CP length for both types of users is $L_{C P}$.

A SC-FDE QPSK modulated uncoded system is simulated. Simulations are carried out with two antenna elements $(M=2)$ with one ICU $(K=1)$ and one synchronous OCI $(P=1)$ or one asynchronous OCI $(Z=1)$. The three-path SUI-5 channel model [SUI01] with average power-delay profile depicted in Figure B.2 is used. A block length of 256 is assumed. In terms of the samples the channel impulse response spans 60 samples while $L_{C P}$ is 64 samples. PCSI is assumed. Figure B.3a shows the average BER linearly equalized curves with synchronous OCI at different average received power levels $(0,5$, 10,15 and $20 \mathrm{~dB}$ below the ICU). Figure B.3b shows results with a corresponding uncoded OFDM system.

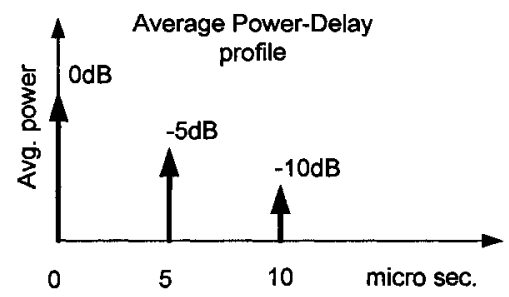

Fig. B.2. Average power-delay profile for SUI-5 model. 


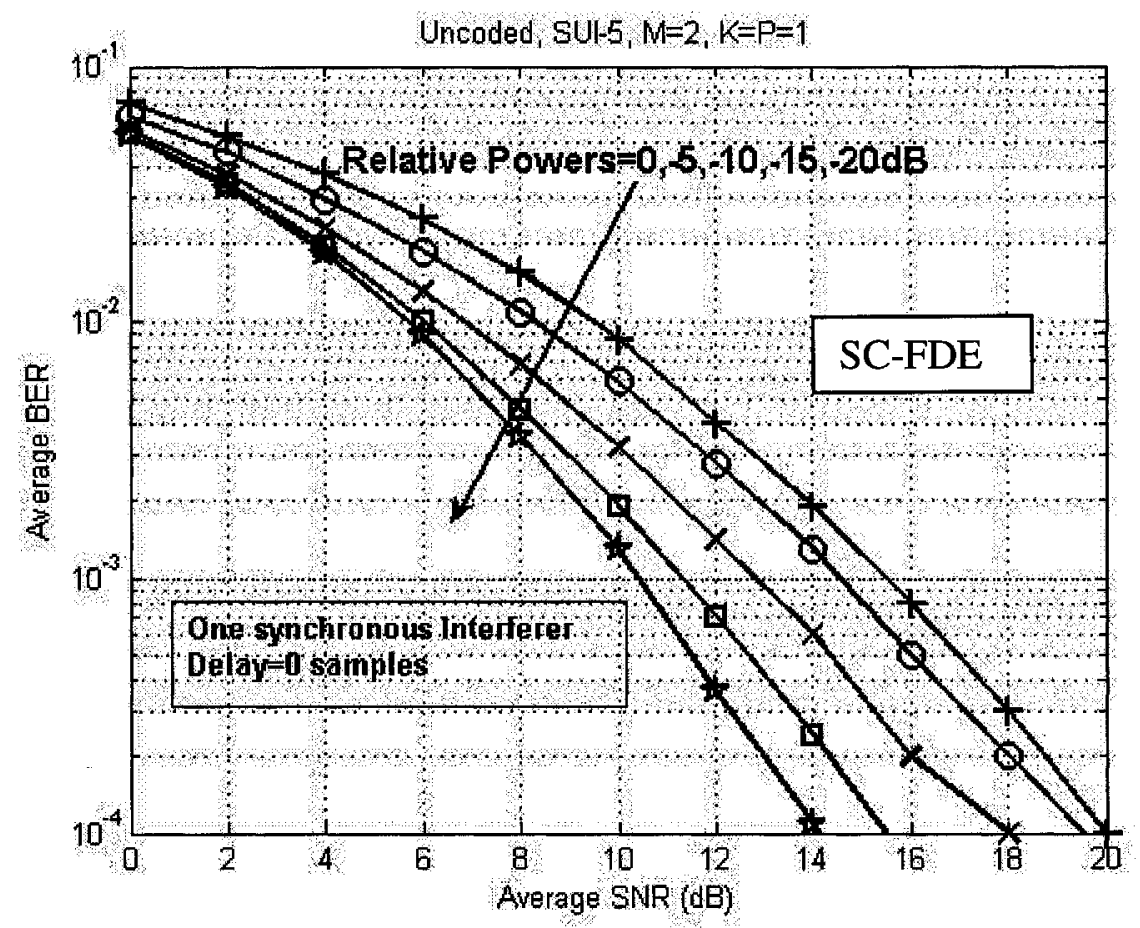

a)

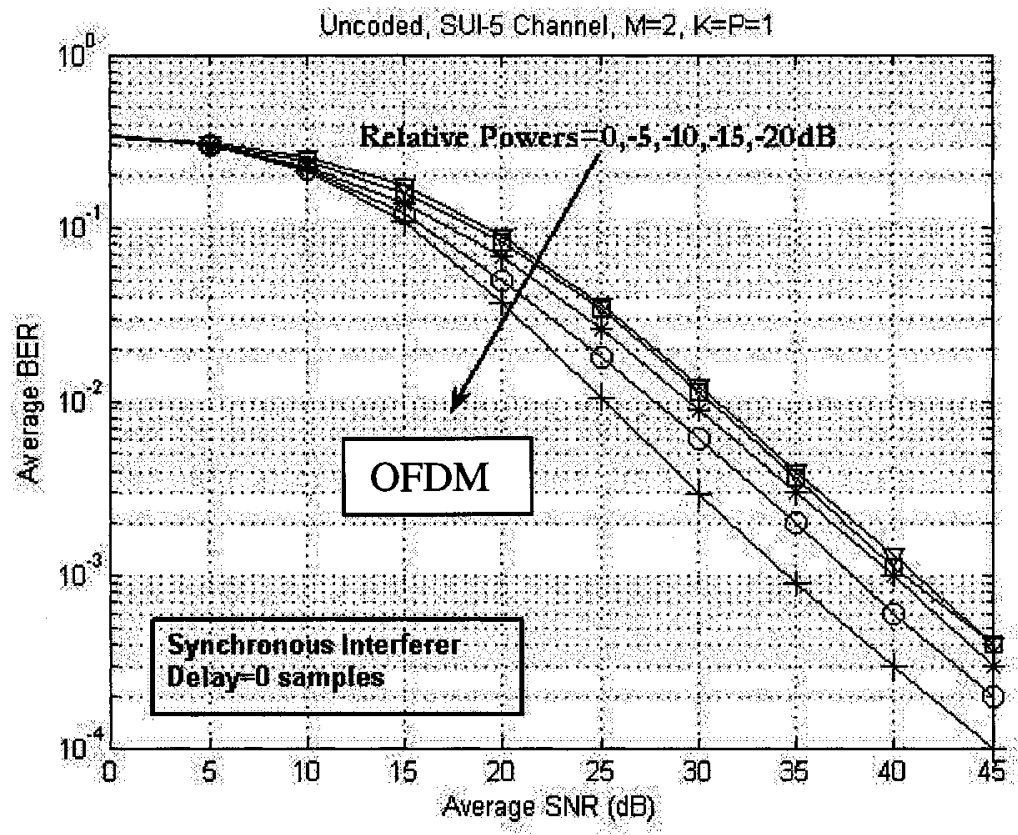

b)

Fig. B.3. a) SC-FDE-Average BER-Synchronous OCI with different power levels, $M=2$, $d_{O C F}=0, K=P=1 @ 0,5,10,15$ and $20 \mathrm{~dB}$ below the ICU. b) OFDM-Average BER (specifications are the same as in part a). 
Figure B.4 illustrates the effect of asynchronous OCI on the performance with different power levels. Again, the simulations are carried out with two antenna elements with one ICU and one AOCI. The delay of the OCI is 60 samples. Figure B.4a shows results with SC-FDE system. In comparison to Figure B.3a, there is an error floor at higher SNR for higher power AOCIs (i.e. OdB and $-5 \mathrm{~dB}$ AOCIs). At higher SNRs, ICI due to asynchronism is causing the floor. A similar trend with OFDM system can be seen in Figure B.4b. 


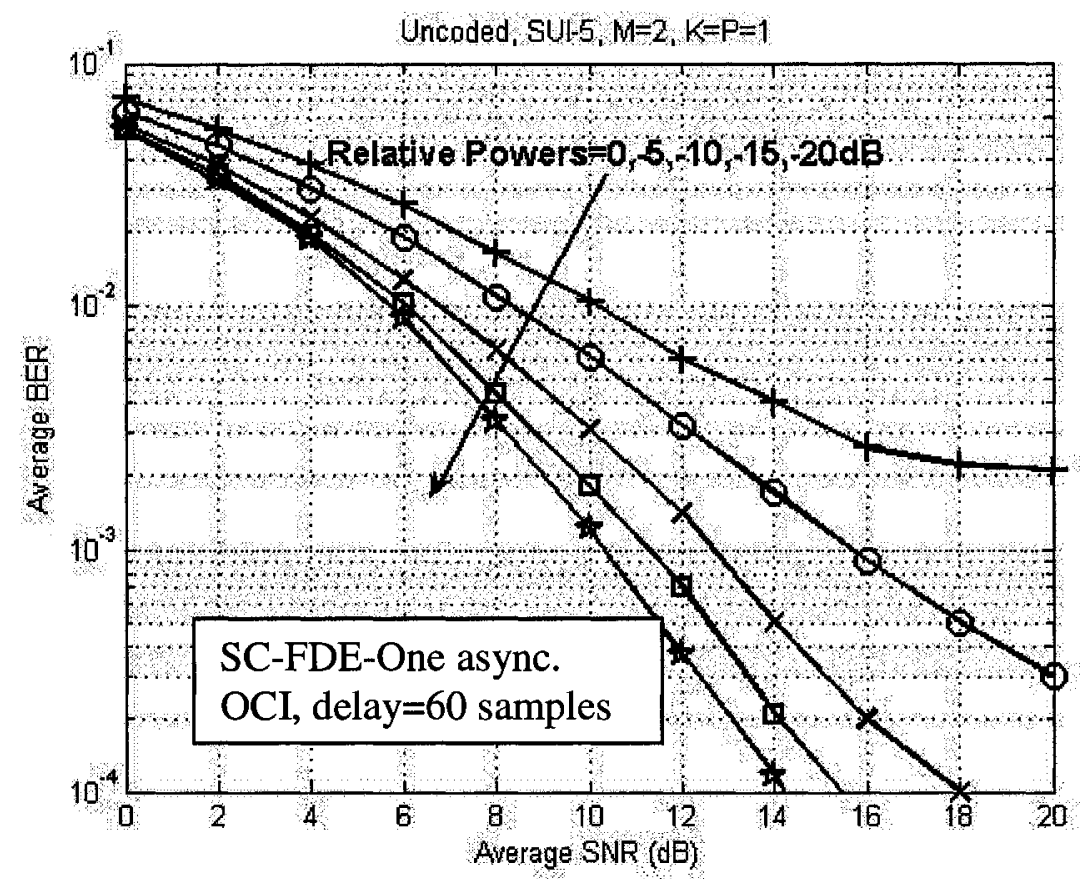

a)

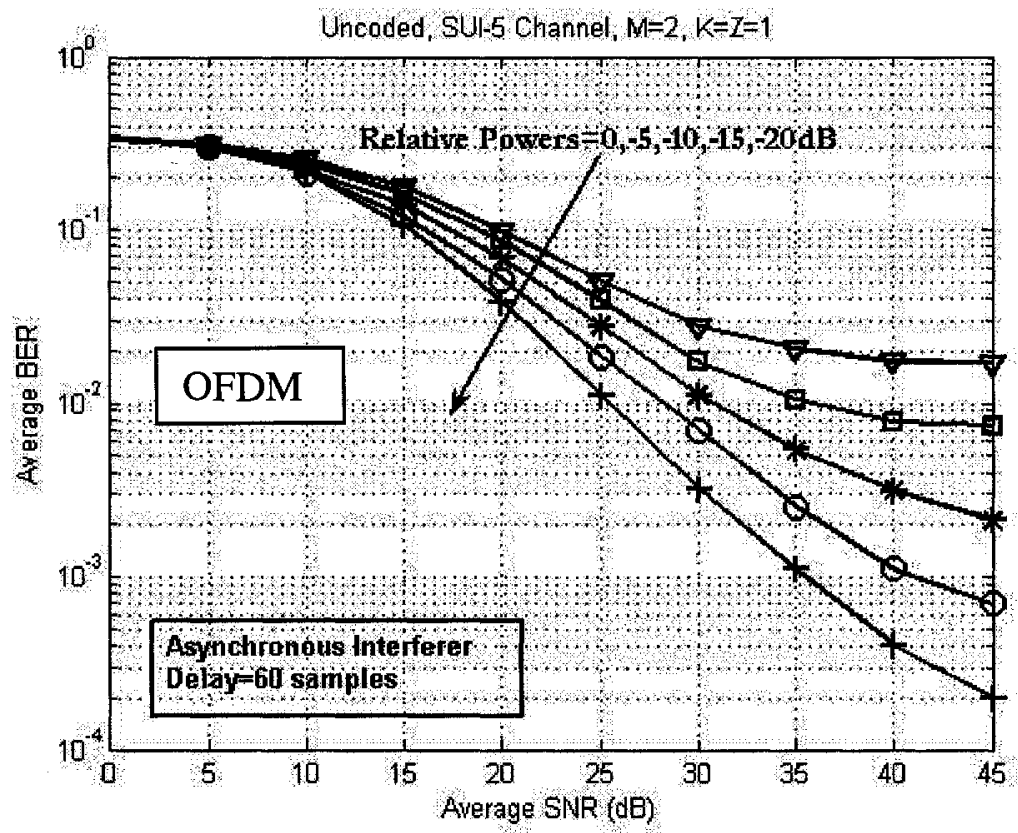

b)

Fig. B.4. a) SC-FDE-Average BER-Asynchronous OCIs with different power levels, $M=2, d_{O C I}=60, K=Z=1$. b) OFDM-Average BER (specifications are the same as in part a). 


\section{B.1.2. Suppression of AOCI}

Removal of the ICI due to asynchronous users in OFDM system has been studied extensively in [MBS02] and [CC93]. One of the techniques to remove the ICI caused by the AOCIs is Time Domain Equalization (TDE) with Channel Shortening (CS). The channel impulse responses for all the users can be shortened into certain time windows of size less than the $\mathrm{CP}$ to accommodate the frequency domain cancellation. The CS problem has been studied for DMT systems [BZT02] and can be applied to multi-user SC-FDE or OFDM systems. The other technique used to reduce the asynchronism effects is known as overlap and cut method [ITK07]. In this section both of the above described techniques are applied to a SC-FDE system with one AOCI.

\section{B.1.2.1. Space-Time Processing to Align AOCI}

Degradation due to the AOCIs with small delays is reduced by designing a TDE that effectively aligns the CPs of the desired and AOCI signals by constraining the lengths of the effective channels from OCIs to the output of the equalizer to be less than or equal to the length of the CP of the ICU. There are two time domain approaches to shorten the CIR. The MMSE CS approach was originally proposed by [FM73] as an optimization criterion for CS in ML receivers. In this approach, a reference or target impulse response transversal filter is used, whose order is equal to the desired impulse response length. The MSE between the output of the reference system and the output of the equalizer is minimized. This approach has been extensively used in the later work in relation to Discrete Multi-Tone (DMT) systems [LCC95] and [CC93]. The second approach presented by [MYR96], limits the length of the effective impulse response of the channel by minimizing the residual ISI. 


\section{B.1.2.1.1. MMSE CS}

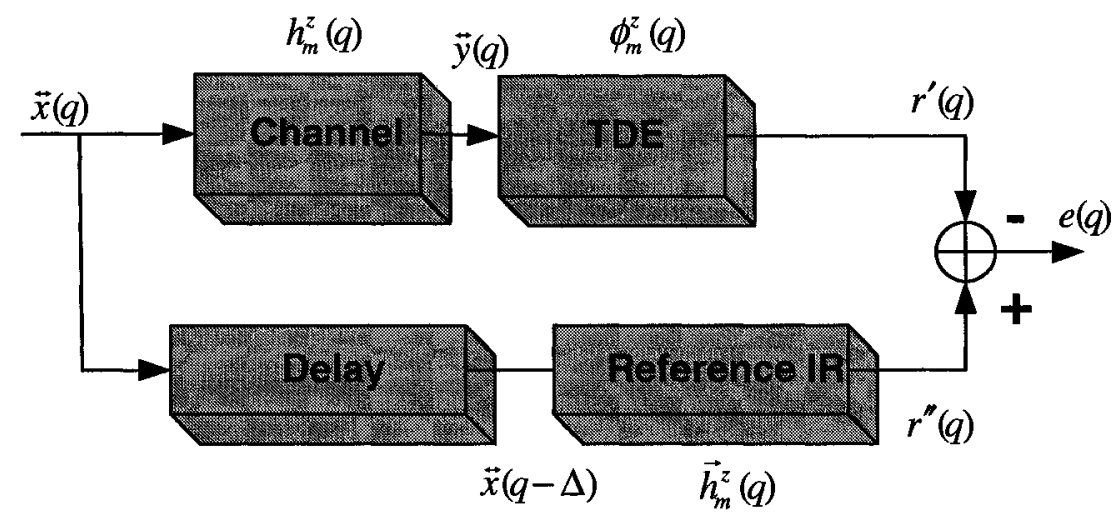

Fig. B.5. MMSE time domain equalization for channel truncation.

A time domain equalizer $\varphi_{m}^{\tau}(q)$, with length $P^{\prime}$ is applied to the AOCI's channel impulse response $b_{m}^{z}(q)$ of length $L$ to get the desired AOCI's channel impulse response $\vec{b}_{m}^{z}(q)$ of length $\vec{L}$. Figure B.5 illustrates a simplified block diagram. A training sequence $\vec{x}(q)$ is sent and an error signal is generated by comparing the reference or target OCIs' impulse response model $\vec{b}_{m}^{\chi}(q)$, with the time domain equalizer $\left(\varphi_{m}^{\chi}(q)\right)$ output. The filter coefficients of $\varphi_{m}^{q}(q)$ and $\vec{h}_{m}^{q}(q)$ are chosen to minimize the MSE value $J_{e r}$ of the error signal $e(q)$. By ignoring the influence of the noise, $J_{e r}$ can be written as [CC93]:

$$
J_{e r}=E\left(e(q)^{2}\right)=E\left(\left(r^{\prime}(q)-r^{\prime \prime}(q)\right)^{2}\right)
$$

where $r^{\prime}$ and $r^{\prime \prime}$ are defined in Figure B.5. From Figure B.5, (B.1) can be written as

$$
J_{e r}=E\left[\left(\sum_{l=0}^{\vec{L}-1} \vec{b}_{m}^{\chi}(l) \vec{x}(q-\Delta-l)-\sum_{l=0}^{P^{\prime}-1} \varphi_{m}^{\tau}(l) \vec{y}(q-l)\right)^{2}\right]
$$

As for uncorrelated training symbols $E(\vec{x}(i) \vec{x}(j))=\delta(i-j)$, where $\delta(i)$ is the Kroneker delta function, (B.2) can be written as:

$J_{e r}=\sum_{l=0}^{\vec{L}-1} \vec{b}_{m}^{z}(l) \vec{b}_{m}^{z}(l)-2 \sum_{l=0}^{\vec{L}-1} \sum_{b=0}^{P^{\prime}-1} \vec{b}_{m}^{z}(l) \varphi_{m}^{z}(b) b_{m}^{z}(l-b+\Delta)+\sum_{n=0}^{P^{\prime}} \sum_{b=0}^{P^{\prime}} \sum_{j=0}^{L-1} \varphi_{m}^{z}(b) \varphi_{m}^{z}(n) h_{m}^{z}(j) h_{m}^{z}(j+n-b)$

In vector notation

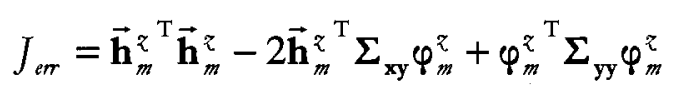


where, $\mathbf{h}_{m}^{z} \in C^{\left(P^{\prime}+L-1\right) x P^{\prime}}$,

$$
\mathbf{h}_{m}^{z}=\left[\begin{array}{lll}
h_{m}^{z}(0) & & 0 \\
\vdots & \ddots & \\
h_{m}^{\tau}(L-1) & \cdots & h_{m}^{z}(0) \\
& \ddots & \\
0 & & h_{m}^{z}(L-1)
\end{array}\right]
$$

and $\Sigma_{\mathrm{xy}} \in \mathrm{C}^{\vec{L} x P^{\prime}}$ and $\Sigma_{\mathrm{yy}} \in \mathrm{C}^{P^{\prime} x P^{\prime \prime}}$ are the channel correlation matrices, given by:

$$
\Sigma_{\mathbf{x y}}(i, j)=b_{m}^{z}(i-j+\Delta)
$$

and

$$
\Sigma_{\mathrm{yy}}(i, j)=\sum_{g=0}^{L-1} h_{m}^{z}(g) h_{m}^{z}(g+i-j)
$$

To minimize the MSE, we differentiate (B.4) and equate it with zero to obtain the optimum filter coefficients as:

$$
\varphi_{m}^{z}=\Sigma_{\mathbf{x y}}^{-1} \overrightarrow{\mathbf{h}}_{m}^{z}
$$

\section{B.1.2.1.2. Minimizing the Residual ISI}

A set of equalizers $\varphi_{m}^{z}(q)$, with lengths $P^{\prime}$ is applied to the AOCI's channel impulse response $b_{m}^{z}(q)$ to get the desired AOCI's channel impulse response $\vec{b}_{m}^{z}(q)$ such as the most of the energy of $\vec{h}_{m}^{z}(q)$ should fall within the window of $\vec{L}$ taps [MYR96] as shown in Figure B.6.

$$
\vec{b}_{m}^{z}(q)=b_{m}^{z}(q) * \varphi_{m}^{z}(q)
$$

In vector notation, $\overrightarrow{\mathbf{h}}_{m}^{z}=\mathbf{h}_{m}^{z} \varphi_{m}^{z}$. 


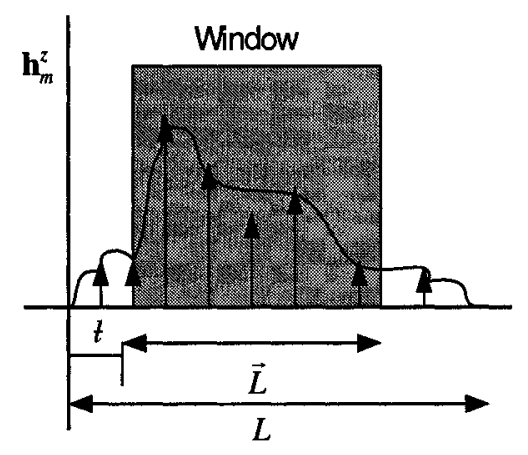

Fig. B.6. Impulse response of the AOCI.

$\overrightarrow{\mathbf{h}}_{m}^{z}$ can be divided into two parts. One covers the samples within the window $\overrightarrow{\mathbf{h}}_{\mathrm{iw}} \in \mathrm{C}^{\vec{L} \times 1}$ as shown in Figure B.6, and the other contains the samples from outside the window $\overrightarrow{\mathbf{h}}_{\text {ow }} \in C^{(L-\vec{L}) \times 1}$. Here and onward we use simpler notation by omitting the user and antenna index. $\overrightarrow{\mathbf{h}}_{\mathrm{iw}}$ and $\overrightarrow{\mathbf{h}}_{\mathrm{ow}}$ are given by:

$$
\begin{aligned}
\overrightarrow{\mathbf{h}}_{\mathrm{iw}} & =\left[\begin{array}{llll}
b_{t} & b_{t-1} & \cdots & b_{t-P^{\prime}+1} \\
b_{t+1} & b_{t} & \cdots & b_{t-P^{\prime}+2} \\
\vdots & \vdots & \ddots & \vdots \\
b_{t+\vec{L}-1} & b_{t+\vec{L}-2} & \cdots & b_{t-P^{\prime}+\vec{L}+1}
\end{array}\right] \\
\overrightarrow{\mathbf{h}}_{\mathbf{o w}} & =\left[\begin{array}{llll}
b_{0} & 0 & \cdots & 0 \\
b_{1} & b_{0} & \cdots & 0 \\
\vdots & \vdots & \ddots & \vdots \\
b_{t-1} & b_{t-2} & \cdots & b_{t-P^{\prime}} \\
b_{t+\vec{L}} & b_{t+\vec{L}-1} & \cdots & b_{t-P^{\prime}+\vec{L}+1} \\
\vdots & \ddots & & \\
b_{L-1} & b_{L-2} & \cdots & b_{L-P^{\prime}} \\
0 & b_{L-1} & \cdots & b_{L-P^{\prime}-1} \\
0 & 0 & \ddots & \\
\vdots & \vdots & & \vdots \\
0 & 0 & \cdots & b_{L-1}
\end{array}\right]
\end{aligned}
$$


Here we define residual ISI, $\tilde{a}$ due to the imperfect choice of $\varphi_{m}^{z}(q)$, and defined as the ratio of the energy outside the window to the energy inside the window and is given by [MYR96]:

$$
\tilde{a}=\frac{\overrightarrow{\mathbf{h}}_{\mathrm{ow}}^{\mathrm{T}} \overrightarrow{\mathbf{h}}_{\mathrm{ow}}}{\overrightarrow{\mathbf{h}}_{\mathrm{iw}}^{\mathrm{T}} \overrightarrow{\mathbf{h}}_{\mathrm{iw}}}
$$

By properly choosing the equalizer $\varphi_{m}^{z}(q), \tilde{a}$ can be minimized. $\tilde{a}$ is also given by:

$$
\tilde{a}=\frac{\varphi^{\mathrm{T}} \overrightarrow{\mathbf{h}}_{\mathrm{ow}}^{\mathrm{T}} \overrightarrow{\mathbf{h}}_{\mathrm{ow}} \varphi}{\varphi^{\mathrm{T}} \overrightarrow{\mathbf{h}}_{\mathrm{iw}}^{\mathrm{T}} \overrightarrow{\mathbf{h}}_{\mathrm{iw}} \varphi}=\frac{\varphi^{\mathrm{T}} \ddot{\mathbf{X}} \varphi}{\varphi^{\mathrm{T}} \ddot{\mathbf{Y}} \varphi}
$$

Optimal shortening is obtained by choosing $\varphi$ to minimize $\overrightarrow{\mathbf{h}}_{\text {ow }}^{\mathrm{T}} \overrightarrow{\mathbf{h}}_{\mathbf{o w}}$ while satisfying the constraint $\overrightarrow{\mathbf{h}}_{\mathrm{iw}}^{\mathrm{T}} \overrightarrow{\mathbf{h}}_{\mathrm{iw}}=1$. This is equivalent to minimizing $\varphi^{\mathrm{T}} \ddot{\mathbf{X}} \varphi$ with the constraint of $\varphi^{\mathrm{T}} \ddot{\mathbf{Y}} \varphi=1$. [MYR96] uses eigen decomposition to obtain the optimum filter coefficients given by:

$$
\varphi_{o p t}=\left(\sqrt{\ddot{\mathrm{Y}}^{\mathrm{T}}}\right)^{-1} \mathbf{1}_{\text {min }}
$$

where, $\mathbf{t}_{\min }$ is the unit length eigen vector corresponding to the minimum eigen-value of the matrix $\vec{\Lambda}$, given by:

$$
\overrightarrow{\mathbf{\Lambda}}=(\sqrt{\ddot{\mathbf{Y}}})^{-1} \ddot{\mathbf{X}}\left(\sqrt{\ddot{\mathbf{Y}}^{\mathrm{T}}}\right)^{-1}
$$

\section{B.1.2.2. Simulation Results}

Figure B.7 illustrates the effect of applying the time domain CS on the AOCIs. The simulations are carried out with two or three antenna elements with one ICU $(K=1)$ and one synchronous $(P=1)$ or one AOCI $(Z=1) @-15 \mathrm{~dB}$. Uncoded average BER curves with synchronous OCI, AOCI with no CS and AOCI with CS are compared. The delay of the AOCI is 60 samples. It is observed that the error floor caused by the AOCI can be eliminated by applying CS. 


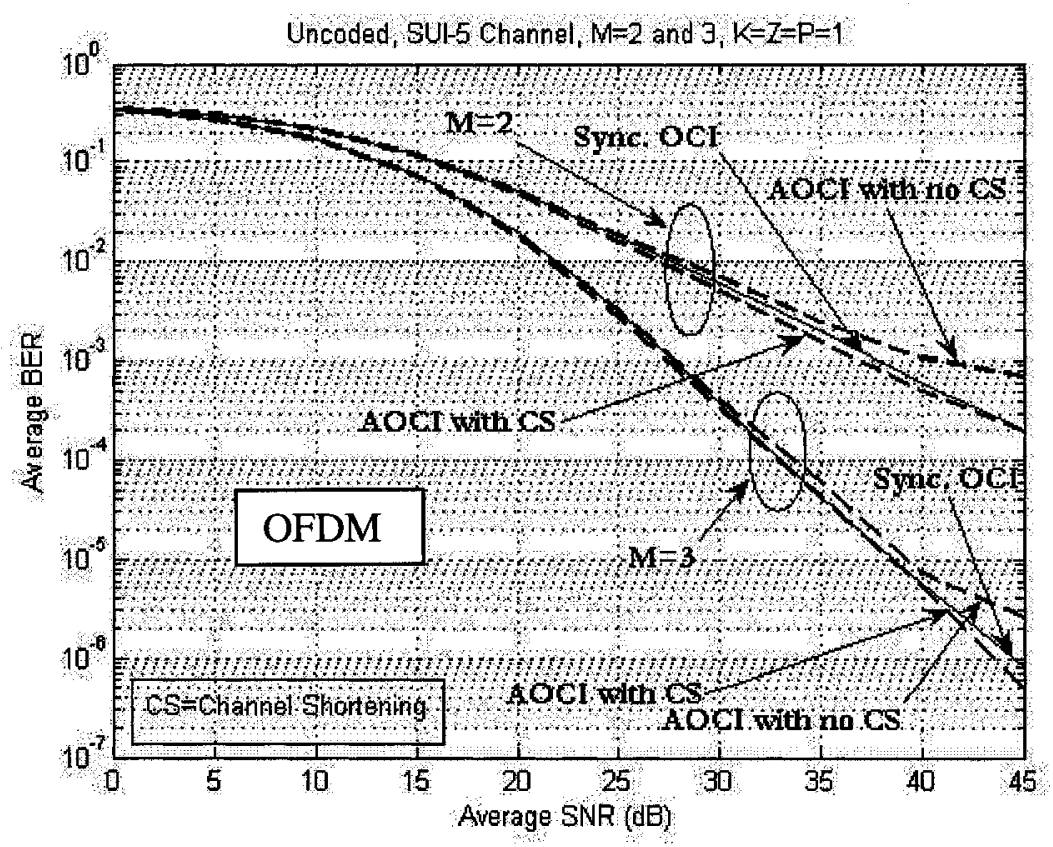

Fig. B.7. Average BER-Effect of low power AOCIs' CS, $M=2$ and 3, $K=P=Z=1 @-15 \mathrm{~dB}$.

It should also be noted that with low power AOCIs, the error floor occurs at higher SNRs and effect of truncation is noticeable only at high SNR values. This suggests that the effect of asynchronism of low power OCIs could be neglected. On the other hand for higher power AOCI, time domain-CS works in the similar way and is effective on all SNRs. This is illustrated in Figure B.8a (SC-FDE) and Figure B.8b (OFDM) with higher power (equal to ICU) AOCI. In these simulations 2 antenna elements are used at the BS. 


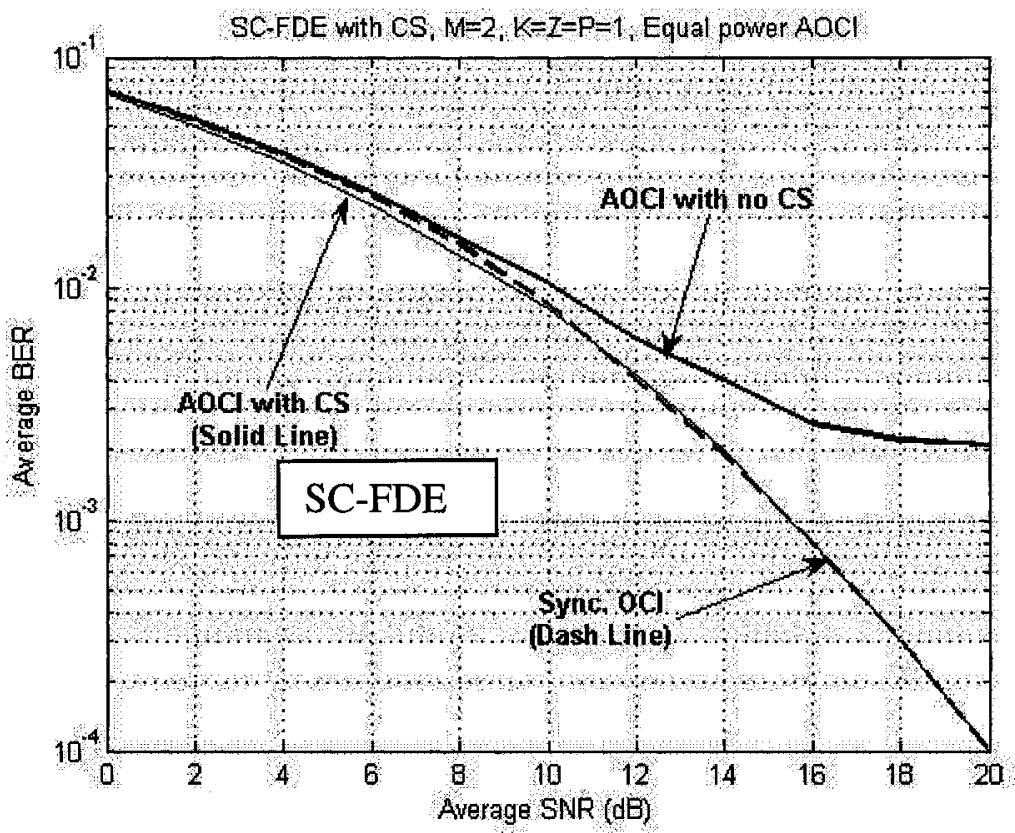

a)

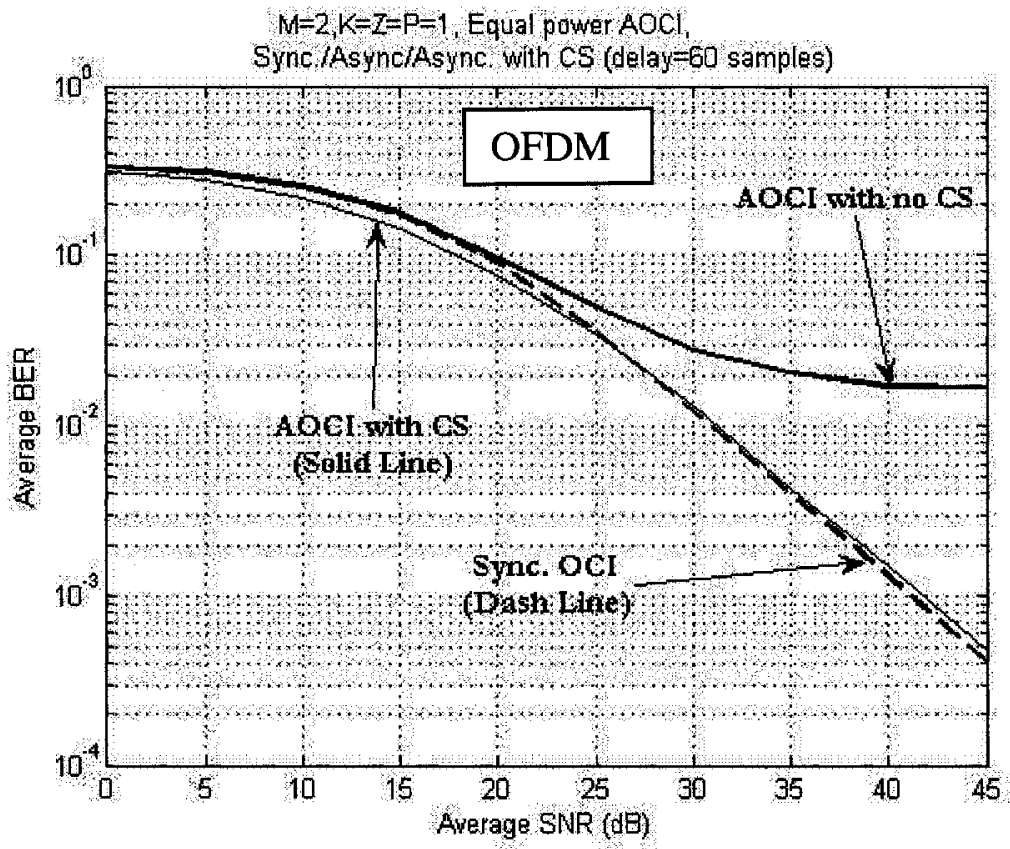

b)

Fig. B.8. a) SC-FDE-Average BER-Effect of higher power AOCIs' CS, $M=2, K=P=Z=1$ @OdB. b) OFDM-Average BER (specifications are the same as in part a). 


\section{B.1.2.3. Overlap and Cut Method}

In this section we apply Overlap and Cut ( $\mathrm{O} \& \mathrm{C})$ method [ITK07] to remove the effects of AOCIs. Figure B.9 illustrates the basic concept associated with the $\mathrm{O} \& \mathrm{C}$ method. In O\&C method, SC-FDE signal is transmitted without a CP. Two overlapping FFTs are performed on the serially received signal. After FFTs the edges are cut to remove the effect of the differences in the arrival times between the ICUs and OCIs.

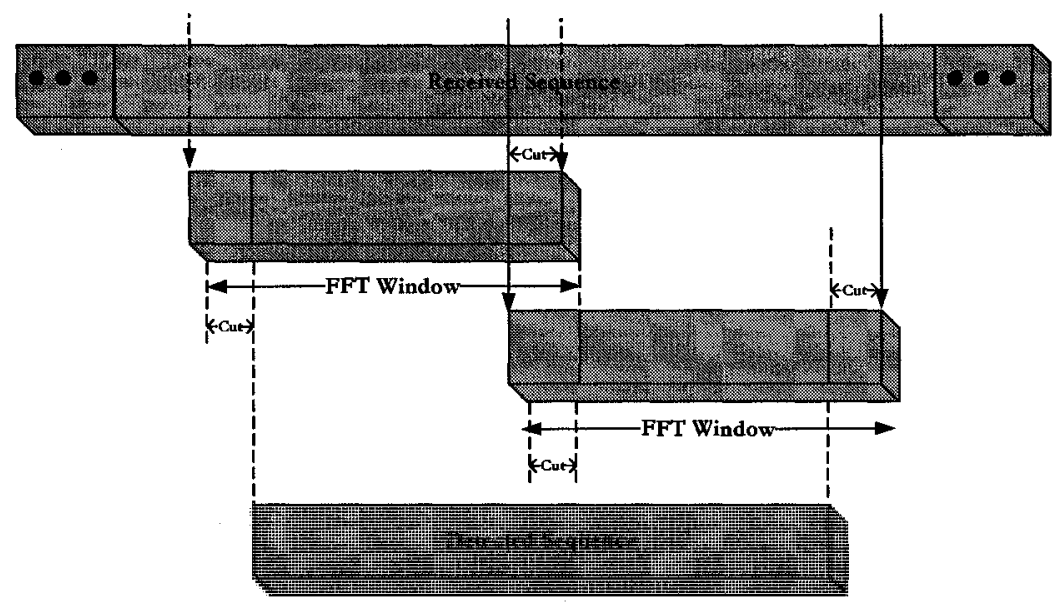

Fig. B.9. Overlap and Cut method.

Simulation results with or without an equal power AOCI are shown in Figure B.10. Simulation parameters are described in Section B.1. Results with conventional CP-based transmission are also shown (Dashed lines) to illustrate the equal performance of the O\&C method with the conventional CP-based technique. In O\&C method as the -transmission is continuous, with reasonable cut-value we may solve the asynchronism problem among different uplink users. The effect of different cut sizes is also shown in Figure B.10. 


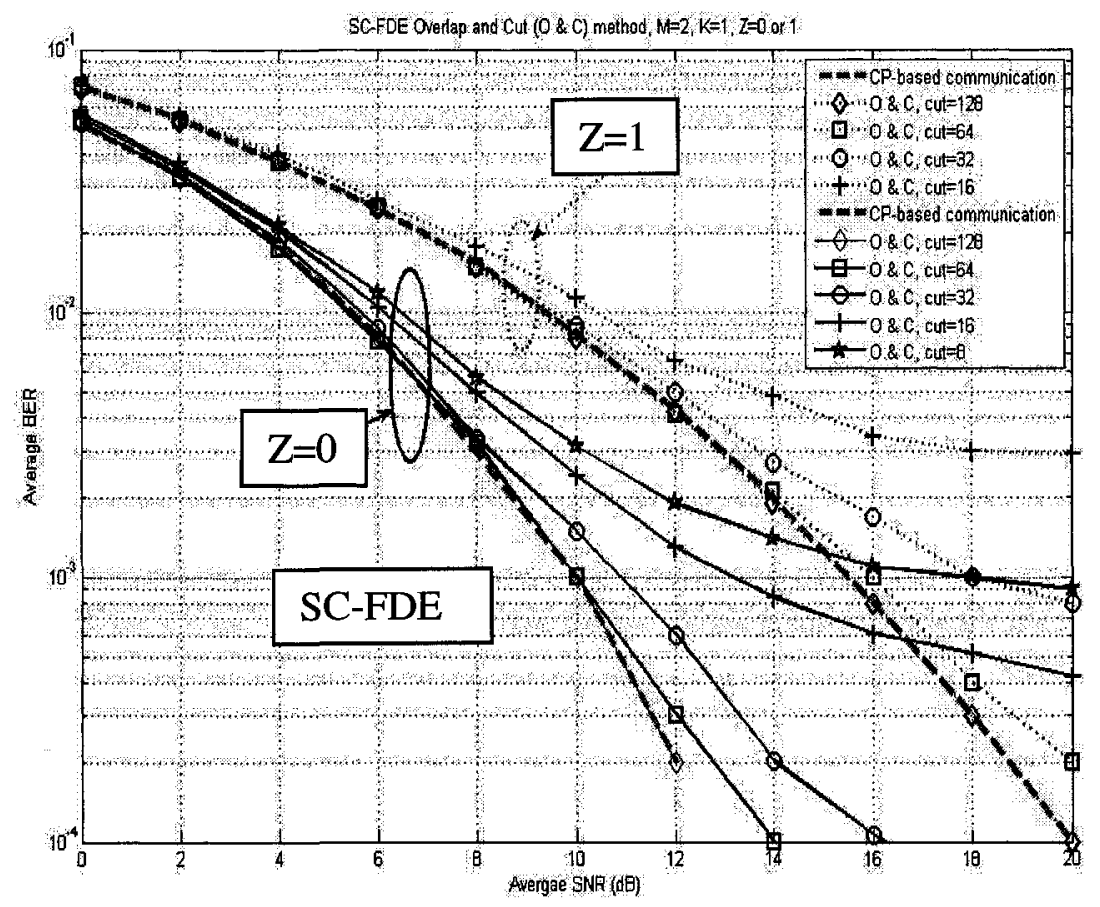

Fig. B.10. SC-FDE-Average BER-Effect of equal power AOCIs' CS, $M=2, K=P=Z=1$ @0dB.

\section{B.2. Conclusions}

It is concluded that the effect of asynchronous OCIs is not severe as far as their power levels are low enough and can be ignored. Although necessary signal processing is necessary if the BS experiences higher power levels of the asynchronous OCIs. 


\section{References}

[AFV98] V. Aue, G.P. Fettweis and R. Valenzuela, "A comparison of the performance of linearly equalized single carrier and coded OFDM over frequency selective fading channels using the random coding technique", IEEE International Conference on Communications, vol. 2, pp.753 - 757, June 1998.

[Ald01] N. Al-Dhahir, "Single-carrier frequency-domain equalization for space-timecoded transmissions over broadband wireless channels", IEEE International Symposium on Personal Indoor and Mobile Radio Communications, vol.1, pp. B-143 - B-146, 30 Sept.-3 Oct. 2001.

[ALM03] K. V. Acker, G. Leus, M. Moonen, T. Pollet, "RLS-based initialization for per tone equalizer in DMT receivers", IEEE Transactions on communications, vol. 51, pp. 885-889, June 2003.

[ATM03] T. Abe, S. Tomisato, T. Matsumoto, "A MIMO turbo equalizer for frequencyselective channels with unknown interference", IEEE Transactions on Vehicular Technology, vol. 52, pp. 476 - 482, May 2003.

[BA06] J. Bonnet, G. Auer, "Optimized Iterative Channel Estimation for OFDM", IEEE Vehicular Technology Conference, vol. 1, pp. 1-5, September 2006.

[BA07] J. Bonnet, G. Auer, "Threshold Controlled Iterative Channel Estimation for Coded OFDM", IEEE Vehicular Technology Conference, vol. 1, pp. 1737-1741, April 2007.

[BHP02] H. Bolcskei, R. Heath, A. Paulraj, "Blind Channel Estimation Identification and Equalization in OFDM-based Multi-Antenna Systems", IEEE Transactions on signal Processing, vol. 50, pp. 96-109, Jan. 2002.

[Bri01] S. T. Brink, "Convergence behavior of iteratively decoded parallel concatenated codes”, IEEE Transactions on communications, vol.49, pp.1727-1737, Oct. 2001.

[BT02a] N. Benvenuto and S. Tomasin, "Block iterative DFE for single carrier modulation", IEEE Electronics Letters, vol. 38, no. 19, pp.1144 - 1145, 12 Sep 2002. 
[BT02b] N. Benvenuto and S. Tomasin, "On the comparison between OFDM and single carrier modulation with a DFE using a frequency-domain feed forward filter", IEEE Transactions on Communications, vol. 50, pp.947 - 955, June 2002.

[BZT02] M. B. Breinholt, M. D. Zoltowski, T. A. Thomas, "space-time Equalization and interference cancellation for MIMO-OFDM", Proceeding of Asil. Conference on signals and computer, vol. 2, pp: 1688-1693, Nov. 2002.

[CBM04a] J. P. Coon, M. A. Beach and J. P. McGeehan, "Minimum mean-square error frequency-domain equalization in unique-word based single-carrier systems", IEEE Electronics Letters, vol. 40, no. 16, pp.1003 - 1005, 5 Aug. 2004.

[CBM04b] J. Coon, M. Beach and J. McGeehan, "Optimal training sequences for channel estimation in cyclic-prefix-based single-carrier systems with transmit diversity", IEEE letters on Signal Processing, vol. 11, pp. 729 - 732, Sept. 2004.

[CCO0] C. Chang and K. Chen, "Frequency-Domain Approach to Multi-user Detection in DS-CDMA Communications", IEEE Communications Letters, vol. 4, pp. 331333, Nov. 2000.

[CC93] J. Chow, J. M. Cioffi, "Equalizer training algorithms for multi-carrier communication systems", IEEE International Conference on Communications, pp. 761-765, 1993.

[Chu72] D. C. Chu, "Polyphase codes with good periodic correlation properties", IEEE Transactions on Information Theory, vol. 18, pp. 531-532, July 1972.

[CL05] J. Chen, S. Li, "Iterative Channel Estimation for MIMO OFDM Systems", International conference on communications, circuits and systems, vol. 1, pp. 180-184, May 2005.

[CM98] G. Caire and U. Mitra, "Training sequence design for adaptive equalization of multi-user systems", Asilomar Conference on Signals, Systems \& Computers, vol. 2, pp. 1479 - 1483, Nov. 1998.

[CS02] M. X. Chang, Y. Su, " Model based channel estimation for OFDM signals in Rayleigh fading", IEEE Transactions on communications, vol. 50, pp. 540-544, April 2002. 
[CS05] J. Coon, M. Sandell, "Designing unique words for reliable channel estimation in multi-antenna block transmission systems", IEEE Workshop on Computational Advances in Multi-Sensor Adaptive Processing, pp.137 - 140, 13-15 Dec. 2005.

[CSB03] J. Coon, J. Siew, M. Beach, A. Nix, S. Armour and J. McGeehan, "A comparison of MIMO-OFDM and MIMO-SCFDE in WLAN environments", IEEE Global Telecommunications Conference, vol. 6, pp.3296 - 3301, 1-5 Dec. 2003.

[CSB04] J. Coon, M. Sandell, M. Beach and J. McGeehan, "Channel and noise variance estimation and tracking algorithms for unique-word based single-carrier systems", IEEE Transactions on Wireless Communications, vol. 5, no. 6, pp. 1488 - 1496, June 2006.

[Czy97] A. Czylwik, "Comparison between adaptive OFDM and single carrier modulation with frequency domain equalization", IEEE Vehicular Technology Conference, vol. 2, pp.865 - 869, 4-7 May 1997.

[Czy98] A. Czylwik, "Low overhead pilot-aided synchronization for single carrier modulation with frequency domain equalization", IEEE Global Telecommunications Conference, vol. 4, pp.2068 - 2073, 8-12 Nov.1998.

[DGE01] L. Deneire, B. Gyselinckx and M. Engels, "Training sequence vs. cyclic prefix a new look on single carrier communication", IEEE Global Telecommunications Conference, vol. 2, pp.1056 - 1060, 27 Nov.-1 Dec. 2000.

[DGE04] R. Dinis, A. Gusmao, N. Esteves, "Iterative block-DFE techniques for singlecarrier-based broadband communications with transmit/receive space diversity", 1st International Symposium on wireless communications, pp. 347-351, September 2004.

[DKF04] R. Dinis, R. Kalbasi, D. Falconer and A.H. Banihashemi, "Iterative layered space-time receivers for single-carrier transmission over severe time-dispersive channels", IEEE Communications Letters, vol. 8, no. 9, pp.579 - 581, Sept. 2004.

[ESB96] O. Edfors, M. Sandell, J. V. Beek, S. K.Wilson and P. Ola Borjesson, "OFDM channel estimation by singular value decomposition", IEEE Vehicular Technology Conference, vol. 2, pp. 923 - 927, May 1996. 
[FAB02] D. Falconer, S.L. Ariyavisitakul, A. Benyamin-Seeyar and B. Eidson, "Frequency domain equalization for single-carrier broadband wireless systems", IEEE Communications Magazine, vol. 40, pp. 58 - 66, April 2002.

[Fer85] E. R. Ferrara, Jr., "Frequency-Domain Adaptive Filtering", Adaptive Filters, C.F.N. Cowan and P.M. Grant, Editors, Prentice-Hall, 1985.

[FM73] D. D. Falconer, F. R. Magee, "Adaptive Channel Memory truncation for maximum likelihood sequence estimation", Bell Systems Technical Journal, pp: 1541-1562, Nov. 1973.

[Gal62] R. G. Gallager, "Low-Density Parity-check code", IRE Transactions on information theory, vol. IT-8, pp. 21-28, Jan 1962.

[GDC00] A. Gusmao, R. Dinis, J. Conceicao, N. Esteves, "Comparison of two modulation choices for broadband wireless communications", IEEE Vehicular Technology Conference Proceedings, vol. 2, pp.1300 - 1305, 15-18 May 2000.

[GL01] Y. Gong, K. Letaief, "Low rank Channel Estimation for space-time coded wideband OFDM Systems", IEEE Vehicular Technology Conference, vol.2, pp. 772-776, Oct. 2001.

[Gun04] Gunther Auer, "Analysis of pilot-symbol aided channel estimation for OFDM systems with multiple transmit antennas", IEEE Conference on Communications, pp. 3221-3225, June, 2004.

[Hay02] S. Haykin, "Adaptive Filter Theory", 4rth Edition, Prentice-Hall, Inc. 2002.

[HH00] A. Hutter, R. Hasholzner, "Determination of Inter-carrier Interference Covariance Matrices and their Application to Advanced Equalization for Mobile OFDM", 5th International OFDM Workshop, Hamburg, pp. 33/1-33/5, Sept. 2000.

[HK06] L. Hanzo, T. Keller, “OFDM and MC-CDMA A Primer”, John Wiley and Sons, Ltd. 2006.

[HKR97] P. Hoeher, S. Kaiser and P. Robertson, “Two-dimensional pilot-symbol-aided channel estimation by Wiener filtering", Conference on Acoustics, Speech, and Signal Processing, vol. 3, pp.1845 - 1848, April 1997. 
[ITK07] K. Ishihara, Y. Takatori, S. Kubota, "Multiuser Detection Method for SingleCarrier Transmission in Uplink Multiuser MIMO Access", IEEE Vehicular Technology Conference, pp.566-570 - 1279, Sept.2007.

[JCC99] W.G. Jeon, K. H. Chang, Y. S. Cho, “An Equalization Technique for orthogonal Frequency-Division Multiplexing Systems in Time-Variant Multipath Channels", IEEE Transactions on Communications, vol. 47, pp. 27-32, Jan. 1999.

[JHS95] Li. Jian, B. Halder, P. Stoica and M. Viberg, "Computationally efficient angle estimation for signals with known waveforms", IEEE Transactions on Acoustics, Speech, and Signal Processing, vol. 43, pp.2154 - 2163, Sept. 1995.

[JPC00] W. Jeon, K. Paik, Y. Cho, " An Efficient Channel Estimation Technique for OFDM systems with Transmitter Diversity", International symposium on Personal, Indoor, and Mobile Radio communications, vol. 2, pp. 1246-1250, Sep. 2000.

[JTN04] A. Jeremic, T. A. Thomas, A. Nehorai, "OFDM Channel Estimation in the Presence of Interference", IEEE Transactions on signal processing, vol. 52, pp. 3429-3439, December 2004.

[Kad97] G. Kadel, "Diversity and Equalization in Frequency Domain; a Robust and Flexible Receiver Technology for Broadband Mobile Communication Systems", IEEE Vehicular Technology Conference, vol. 2, pp.894 - 898, 4-7 May 1997.

[KCC99] C. Kim, S. Choi, Y. Cho, "Adaptive Beam forming for an OFDM System", IEEE Vehicular Technology Conference, May 1999.

[KDF04] R. Kalbasi, R. Dinis, D.D. Falconer and A.H. Banihashemi, "Layered spacetime receivers for single-carrier transmission with iterative frequency-domain equalization", IEEE Vehicular Technology Conference, vol. 1, pp.575 - 579, 17-19 May 2004.

[KDF04a] R. Kalbasi, R. Dinis, D. Falconer and A. Banihashemi, "An iterative frequency-domain layered space-time receiver for SDMA systems with singlecarrier transmission", IEEE International Conference on Acoustics, Speech and Signal Processing, vol. 4, pp.iv-793 - iv-796, 17-21 May 2004. 
[KFS06] T. Kashima, K. Fukawa, H. Suzuki, "Recursive Least Squares Channel Estimator with Smoothing and Removing for Iterative-MAP Receiver of MIMO-OFDM Mobile Communications", IEEE International Conference on Communications, vol. 7, pp. 3052-3057, June 2006.

[KHS03] A. Koppler, M. Huemer, A. Springer and R.Weigel, "Iterative combined decision feedback equalization and decoding for broadband wireless single carrier systems", International Conference on Telecommunications, vol.1, pp.743-747, 23 Feb.-1 March 2003.

[Kor03] J. Korhonen, "Introduction to 3G Mobile Communications", Norwood, U.S.A., Artech House Inc., 2nd Ed. 2003.

[KSN03] K.Y. Kuan, S. Suthaharan and A. Nallanathan, "An adaptive channel estimation scheme for OFDM system with transmitter diversity", IEEE Global Telecommunications Conference, vol. 2, pp. 873-877, 1-5 Dec. 2003.

[KV94] G. K. Kaleh, R. Vallet, "Joint parameter estimation and symbol detection for linear or nonlinear unknown channels" IEEE Transactions on Wireless Communications, vol. 42, no. 7, pp. 2406-2413, July, 1994.

[LCC95] I. Lee, J. Chow, J. M. Cioffi, "Performance evaluation of a fast computation algorithm for the DMT in high speed subscriber loop", IEEE Transactions on selected area on communications, vol. 13, pp. 1564-1570, Dec. 1995.

[LCS98] Y. Li, L. Cimini, N. Sollenberger, "Robust Channel Estimation for OFDM systems with rapid dispersive fading channels", IEEE Transactions on communications, vol. 46, pp. 902-915, Feb. 1999.

[LDF06] C-T Lam, F. Danilo-Lemoine, D. Falconer and R. Dinis, "Channel estimation for SC-FDE Systems Using Frequency Domain Multiplexed Pilots", IEEE conference on Vehicular Technology, Sept., 2006.

[LFD08] C. T. Lam, D.D. Falconer, F. Danilo-Lemoine, "Iterative frequency domain channel estimation for DFT-precoded OFDM systems using in-band pilots", IEEE Journal on Selected Areas in Communications, vol.26, pp. 348-358, April 2007. 
[LGL01] C. Loat, A. Glavieus, J. Labat, "Turbo Equalization: Adaptive Equalization and Channel Decoding Jointly Optimized", IEEE Transactions on selected areas in communications, vol. 19, Sept. 2001.

[Li00b] Y. Li, "Optimum training sequences for OFDM systems with Multiple Transmit Antennas", IEEE Global Telecommunications Conference, vol. 3, pp. 14781482, Dec. 2000.

[Li02] Y. Li, "Simplified channel estimation for OFDM systems with multiple transmit antennas," IEEE Transactions on Communications, vol. 1, no. 1, pp. 67-75, Jan. 2002.

[LS05] S. Lee and A.C. Singer, "Linear Turbo Equalization Analysis via BER Transfer and Exit Charts", IEEE Transactions on signal processing, vol.53, pp.28832897, August. 2005.

[LS98] Y. Li, N. Sollenberger, "Interference Suppression in OFDM Systems using Adaptive Antenna Arrays", IEEE Global Telecommunications Conference, pp. 213-218, Nov. 1998.

[LS99] Y. Li, N. Sollenberger, "Adaptive Antenna Arrays for OFDM Systems with Cochannel Interference", IEEE Transactions on communications, vol. 47, pp. 217229, Feb. 1999.

[LSA99] Y. Li, N. Seshadri, S. Ariyavisitakul, "Channel Estimation for OFDM Systems with Transmitter Diversity in Mobile Wireless Channels", IEEE Transactions on selected area on communications, vol. 17, pp. 461-471, March 1999.

[LWL02] B. Lu, X. Wang, and Y. G. Li, "Iterative receivers for space-time block coded OFDM systems in dispersive fading channels," IEEE Transactions on Wireless Communications, vol. 1, pp. 213-225, Apr. 2002.

[LWL07] B. Lu, X. Wang, and Y. G. Li, "Comparison of EM-Based Algorithms for MIMO Channel Estimation" IEEE Transactions on Wireless Communications, vol. 55, no. 2, pp. 216-226, Jan. 2007.

[LWS02]Y. Li, J. H. Winters, and N. R. Sollenberger, "MIMO-OFDM for wireless communications: Signal detection with enhanced channel estimation," IEEE Transactions on communications, vol. 50, pp. 1471-1477, Sep. 2002. 
[MBS02] R. K. Martin, J. Balak, W. A. Sethares, C.R. Johnson, "A blind adaptive TEQ for multi-carrier systems", IEEE Signal processing Letter, vol. 9, pp. 341-343, Nov 2002.

[MH00] M. Munster, L. Hanzo, "Co-channel interference cancellation techniques for antenna array assisted multiuser OFDM systems", First International Conference on 3G Mobile Communication Technologies, pp. 256-260, 2000.

[MH05] M. Munster, L. Hanzo, "Parallel-interference-cancellation-assisted decisiondirected channel estimation for OFDM systems using multiple transmit antennas", IEEE Transactions on wireless communications, vol. 4, pp. 21482162, Sept. 2005.

[MKB02] H. Minn, D. Kim, V. Bhargava, "A reduced Complexity channel estimation for OFDM systems with Transmit Diversity in mobile wireless channels", IEEE Transactions on wireless communications, vol. 50, pp. 799-807, May 2002.

[MKH99] M. Munster, T. Keller, L. Hanzo, "Co-channel interference suppression assisted adaptive OFDM in interference limited environments", IEEE Vehicular Technology Conference, Sep. 1999.

[MYR96] P. J. W. Melsa, R. C. Younce, C. E. Rohrs, "Impulse Response Shortening for Discrete Multitone Transceivers", IEEE Transactions on communications, vol. 44, pp. 1662-1672, Dec. 1996.

[NES97] R. Nisson, O. Edfors, M. Sandell, P. Borjesson, "An analysis of twodimensional pilot-symbol assisted modulation for OFDM", Proc. IEEE ICPWC, Mumbai, pp. 71-74, 1997.

[NLF07] B. Ng, C-T Lam and D. Falconer, "Turbo Frequency Domain Equalization for Single Carrier Broadband Wireless Systems", IEEE Transactions on Wireless Communications, vol. 6, no. 2, February 2007.

[Par04] R. Parasad, “OFDM for Wireless communication systems”, Artech House 2004.

[Pro95] J.G. Proakis, "Digital Communications", New York, McGraw-Hill, 3rd Edition 1995.

[PVM95] T. Pollet, M. Van-Bladel, M. Moeneclaey, "BER sensitivity of OFDM systems to carrier frequency offset and Wiener phase noise", IEEE Transactions on communications, vol. 43, pp. 191-193, April. 1995. 
[QYS05] Y. Qiao, S. Yu, P. Su, and L. Zhang, "Research on an Iterative Algorithm of LS Channel Estimation in MIMO OFDM Systems", IEEE transactions on broadcasting, vol. 51, March 2005.

[RW02] D. Reynolds and X. Wang, "Turbo Multi-user Detection with Unknown Interferers", IEEE Transactions on communications, vol. 50, pp. 616-622, April 2002.

[SBS97] U. Sorger, I. De Broek, M. Schnell, "IFDMA- A New Spread Spectrum Multiple Access Scheme", Multi-carrier spread spectrum Netherlands: Kluwer Academic Publishers, pp: 111-118, 1997.

[SCP04] J. Siew, J. Coon, R.J. Piechocki, A. Dowler, A. Nix, M. Beach, S. Armour, J. McGeehan, "A channel estimation algorithm for MIMO-SCFDE", IEEE Communications Letters, vol. 8, no. 9, pp. 555 - 557, Sept. 2004.

[SDF07a] F. Siddiqui, F. Danilo-Lemoine and D. Falconer, "On Interference in Uplink SDMA SC-FDE System", IEEE Communication Networks and Services Research Conference, Fredericton, New Brunswick, May14-17, 2007.

[SDF07b] Fayyaz Siddiqui, Florence Danilo-Lemoine and David Falconer, "PIC assisted IBDFE based Iterative Spatial Channel Estimation with Intra and Inter-Cell Interference in SC-FDE System" Proceedings of Vehicular Technology Conference, Baltimore, Sept., 2007.

[SDF08a] F. Siddiqui, F. Danilo-Lemoine and D. Falconer, "Channel Estimation using Soft Decision Feedback in SDMA SC-FDE Systems", submitted to IEEE Transactions on Communications.

[SDF08b] F. Siddiqui, F. Danilo-Lemoine and D. Falconer, "Turbo Equalization based Iterative Channel Estimation in SDMA SC-FDE Systems", WWRF20-WG4-03, Ottawa, ON. 22-24 April 2008.

[SDF08c] F. Siddiqui, F. Danilo-Lemoine and D. Falconer, "Iterative Interference Cancellation and Channel Estimation for Mobile SC-FDE Systems", accepted in IEEE Communications Letter CL2008-0262.

[SF06] M. Sabbaghian, D. D. Falconer, "Comparison between Convolutional and LDPC Code based Turbo Frequency Domain Equalization", IEEE International conference on communications, vol. 1, pp. 5432-5437, June 2006. 
[SFF07] T. Svensson, T. Franky, D. Falconer, M. Sternad, E. Costa, A. Klein, "BIFDMA - A Power Efficient Multiple Access Scheme for Non-frequencyadaptive Transmission”, IST Summit conference, Budapest, July 2007.

[SJS03] F. Sanzi and S. Jelting, J. Speidel, "A comparative study of iterative channel estimators for mobile OFDM systems", IEEE Transactions on Wireless Communications, vol. 2, pp.849-859, Sept. 2003.

[SKJ95] H. Sari, G. Karam and I. Jeanclaude, "Transmission Techniques for Digital Terrestrial TV Broadcasting", IEEE Communications Magazine, vol. 33, no. 2, pp. 100-109, Feb. 1995 ,

[Sli02] S. Slimane, "Channel Estimation for HIPERLAN/2 with Transmitter Diversity", IEEE International Conference on Communications, April 2002.

[SLS98] M. Sandell, C. Luschi, P. Strauch, R. Yan, "Iterative channel estimation using soft decision feedback", IEEE Global Telecommunications Conference, vol. 6, pp. 3728-3733, 1998.

[SNT03] J. G. Sheng, A. Nallanathan and T. T. Tjhung, "Computationally efficient channel estimation for space-time block coded system", IEEE Vehicular Technology Conference, vol. 4, pp. 2614 - 2617, April 2003.

[SRA05] S. Sand, R. Raulefs, G. Auer, "Iterative channel estimation for MC-CDMA", IEEE Vehicular Technology Conference, vol. 1, pp. 471-475, April 2005.

[SSM99] M. Speth, A. Senst, H. Meyr, " Low complexity space-frequency MLSE for multi-user COFDM", IEEE Global Telecommunications Conference, vol. 5, pp. 2395-2399, 1999.

[SUI01] IEEE 802.16.3c-01/29r4, July 16, 2001.

[SYS03] Y. Sun, M. Yee, M. Sandell, "Iterative Channel Estimation with MIMO MMSETurbo Equalization", IEEE Vehicular Technology Conference, vol. 2, pp. 12781282, Oct. 2003.

[Taf06] R. Tafazolli, "Technologies for the wireless Future", Wilreless World Research Forum (WWRF), West Sussex, England, John Wiley \& Sons Ltd., vol. 2, 2006.

[TB04] S. Tomasin and N. Benvenuto, "A reduced complexity block iterative DFE for dispersive wireless applications", IEEE Vehicular Technology Conference, vol. 3, pp.1693 - 1697, Sept. 2004. 
[TCP01] J. Tubbax, B. Come, L. V. Perre, L. Deneire, S. Donnay and M. Engels, “OFDM versus Single Carrier with Cyclic Prefix: a system-based comparison”, IEEE Vehicular Technology Conference, vol. 2, pp.1115 - 1119, 7-11 Oct. 2001.

[TIA04] K. Takeda, T. Itagaki and F. Adachi, "Application of space-time transmit diversity to single-carrier transmission with frequency-domain equalization and receive antenna diversity in a frequency-selective fading channel", IEE Proceedings on Communications, vol.151, no. 6, pp.627 - 632, 24 Dec. 2004.

[TKS02] M. Tuchler, R. Koetter and A. C. Singer, "Turbo Equalization: Principles and New Results", IEEE Transactions on Communications, vol. 50, no. 5, pp.754767, May 2002.

[TM97] F. Tufvesson, T. Maseng, "Pilot assisted channel estimation for OFDM in mobile cellular systems", IEEE Vehicular Technology Conference, vol.3, pp. 1639 1643, 4-7 May 1997.

[TPD03] J. Tubbax, L. V. Perre and S. Donnay, Engels, "Single-carrier communication using decision-feedback equalization for multiple antennas", IEEE International Conference on Communications, vol. 4, pp. 2321 - 2325, May 2003.

[TSK02] M. Tuchler, A. Singer and R. Koetter, "Minimum Mean Squared Error Equalization using a-priori information", IEEE Transactions on Signal Processing, vol. 50, pp. 673-678, March 2002.

[TV03] T. A. Thomas, F. W. Vook, "Asynchronous interference suppression in broadband CP communications", IEEE International conference on communications and networking, pp. 568-572, March 2003.

[VPE99] P. Vandenameele, V. D. Perre, L. Engels, M. De Man, "A novel class of uplink OFDM/SDMA algorithms for WLAN", IEEE Global Telecommunications Conference, vol. 1a, pp. 1-10, 1999.

[Win01] WINNER Project deliverable D5.4 Final report on link level and system level channel models, v.1.4 https://www.ist-winner.org/publicdeliverables.htm. Nov. 2005

[Win02] WINNER IST-2003-507581, D2.2-v1.0, Report on Feasibility of Multi-band transmission Oct. 2004. https://www.ist-winner.org/. 
[Win03] IST-4-027756 WINNER II, "D2.3.1 Channel Estimation and Measurements for the WINNER system," February 2007.

[Win04] IST-4-027756 WINNER II, "D2.3.3 Link Level Procedures for WINNER system," November 2007.

[Win05] IST-4-027756 WINNER II, "D4.7.2 Interference Avoidance Concepts", June, 2007.

[WKW04] H. Witschnig, M. Kemptner, R. Weigel and A. Springer, "Decision feedback equalization for a single carrier system with frequency domain equalization - an overall system approach", 1st International Symposium Wireless Communication Systems, pp. 26 - 30, Sept. 2004.

[WL00] W. Wang, K. Liu, "OFDM channel estimation based on time-frequency polynomial model of fading multipath channel", IEEE Vehicular Technology Conference, vol.1, pp. 460-464, Oct 2001.

[WP98] X. Wang, H. Poor, "Robust Adaptive Array for wireless Communications", IEEE Transactions on communications, vol. 16, pp. 1352-1366, Oct. 1998.

[WS73] T. Walzman and M. Schwartz, "Automatic equalization Using the Discrete Frequency Domain", IEEE Transactions on Info. Theory, vol. IT-19, pp. 59-68, Jan 1973.

[WSW99] H. Witschnig, G. Strasser, R. Weigel, A. Springer, "Antenna Diversity Techniques for a Single Carrier System with Frequency Domain Equalization An Overview", (Institute for Communications and Information Engineering, University of Linz, Austria) and (Institute for Technical Electronics University of Erlangen, Germany).

[XG01] Y. Xie, C. Georghiades, "An EM-based channel estimation algorithm for OFDM with Transmitter Diversity", IEEE Global Telecommunications Conference, vol. 2, pp. 871-875, Nov. 2001.

[XG03] Y. Xie and C. N. Georghiades, "Two EM-type channel estimation algorithms for OFDM with transmitter diversity," IEEE Transactions on Communications, vol. 51, no. 1, pp. 106-115, Jan. 2003.

[YA97] Z. Yuping, H. Aiping, "A novel channel estimation method for OFDM mobile communication systems based on pilot signals and transform-domain 
processing", IEEE Vehicular Technology Conference, vol. 3, pp. 2089 - 2093,47 May 1997.

[YB00] D. J. Young, N. C. Beaulieu, "The generation of correlated Rayleigh random variates by inverse discrete Fourier transform", IEEE Transactions on Communications, vol. 48, no. 7, pp.1114 - 1127, July 2000.

[YCL01a] B. Yang, Z. Cao, K. Letaief, "Analysis of low-complexity Windowed DFT based MMSE Channel Estimator for OFDM systems", IEEE Transactions on communications, vol. 49, pp. 1977-1987, Nov. 2001.

[YCL01b] B. Yang, Z. Cao, K. Letaief, "Low complexity channel estimator based on Windowed DFT and scalar Wiener Filter for OFDM", IEEE International Conference on Communications, pp. 1643-1647, June 2001.

[YLC99] B. Yang, K. Letaief, R. Cheng, Z. Cao, "Robust and improved channel estimation for OFDM systems in Frequency selective fading channels", IEEE Global Telecommunications Conference, vol. 5, pp. 2499-2503, Dec. 1999.

[YSS04] M. S. Yee, M. Sandell and Y. Sun, "Comparison study of single-carrier and multi-carrier modulation using iterative based receiver for MIMO system", IEEE Vehicular Technology Conference, vol.3, pp.1275 - 1279, May 2004.

[Zie91] R. A. Ziegler, "Digital Communication over Fading and Dispersive Channels, with Emphasis on Mobile Radio Applications", Dissertation submitted to Department of EE of Stanford University, Sept. 1991.

[ZP99] H. Zamiri-Jafarian, S. Pasupathy, "EM-Based Recursive Estimation of Channel Parameters", IEEE Transactions on communications, vol. 47, pp. 1297-1302, Sept. 1999. 